Supplementary Materials for

\title{
Organocatalytic Synthesis of $\alpha$-Trifluoromethyl Allylboronic Acids by Enantioselective 1,2-Borotropic Migration
}

Sybrand J. T. Jonker ${ }^{1 \dagger}$, Ramasamy Jayarajan ${ }^{1 \dagger}$, Tautvydas Kireilis ${ }^{1}$, Marie Deliaval ${ }^{1}$, Lars Eriksson $^{2}$, Kálmán J. Szabó ${ }^{1 *}$

$\dagger$ These authors contributed equally to this work

*Corresponding author. E-mail: kalman.j.szabo@su.se

This PDF file includes:

Materials and Methods

Supplementary Text

Figs. S1 to S6

NMR Spectra

References 


\section{Table of Contents}

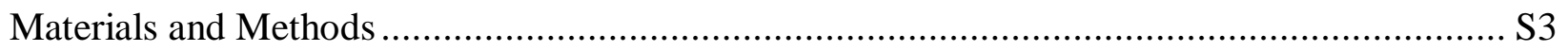

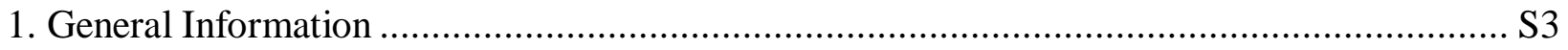

2. Protection of allylboronic acids for isolation........................................................... S4

3. Experimental Procedures and Spectral data ............................................................... S5

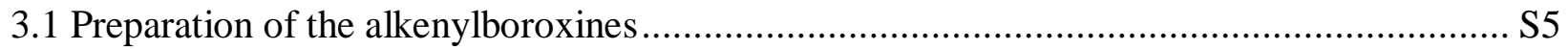

3.2 General procedure A: Asymmetric homologation of vinyl boronic acid ..........................S8

3.3 General procedure B: Asymmetric homologation of vinyl boronic acid using TMS

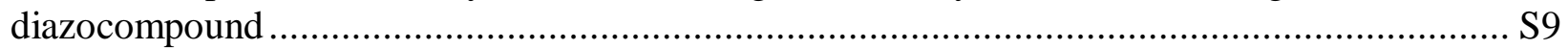

3.4 General Procedure C: One-pot allylboration of aldehyde 6a ..................................... S9

3.5 General Procedure D: In situ oxidation of allylboronic acids ....................................... S9

3.6 General procedure E: Hydrolysis of BDan and allylboration reaction with isolated

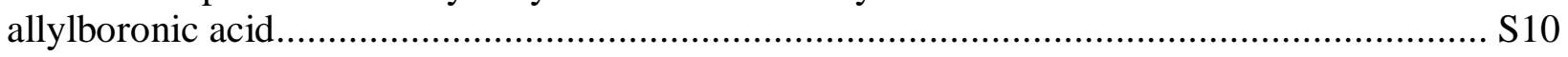

3.7 Stereoinduction model for the homologation reaction ....................................... S20

3. 8 Determination of the absolute configuration: Mosher ${ }^{1} \mathrm{H}$ NMR method ........................ S27

3.9 NMR spectra of purified allylboronic acids ........................................................... S44

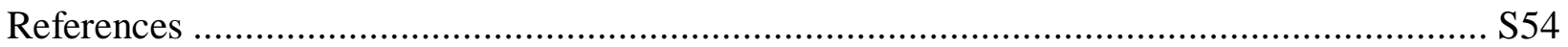

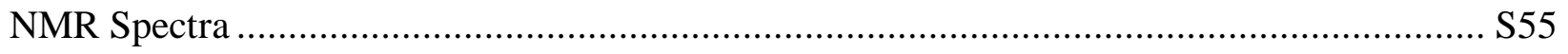




\section{Materials and Methods}

\section{General Information}

Reagent information: Unless otherwise stated, all reactions were carried out under argon atmosphere in screw cap reaction tubes. All vinylboronic acids were purchased from commercial sources, except (E)-(4-bromostyryl)boronic acid which was synthesized according to methods previously reported in literature. ${ }^{1} \mathrm{CF}_{3}$-diazocompound was synthesized in DCM following a reported procedure. ${ }^{2}$ It was stored in DCM over activated molecular sieves ( $3 \AA$ pellets) in the freezer and used for the reaction. TMS-diazocompound was purchased from commercial source (2 $\mathrm{M}$ in $\mathrm{Et}_{2} \mathrm{O}$ ). (R)-3,3'-diiodo-[1,1'-binaphthalene]-2,2'-diol was prepared by reported procedure. ${ }^{3}$ Dry degassed DCM, toluene and activated molecular sieves ( $3 \AA$ pellets) were stored in an argonfilled glove box. For column chromatography, silica gel (35-70 microns) was used. TLC was performed on aluminium backed plates pre-coated $(0.25 \mathrm{~mm})$ with Silica Gel 60 F254 with a suitable solvent system and was visualized using UV fluorescence and/or developed with permanganate stain.

Analytical Information: All isolated compounds were characterized by ${ }^{1} \mathrm{H},{ }^{13} \mathrm{C},{ }^{19} \mathrm{~F}$ and ${ }^{11} \mathrm{~B}$ NMR spectroscopy using Bruker $400 \mathrm{MHz}$ and $500 \mathrm{MHz}$ spectrometers. Copies of the NMR spectra can be found in the end of this Supporting Material. All ${ }^{1} \mathrm{H}$ NMR experiments were reported in units, parts per million (ppm), and were measured relative to the signals for residual chloroform (7.26 $\mathrm{ppm}$ ) in the deuterated solvent, unless otherwise stated. All ${ }^{13} \mathrm{C}$ NMR spectra were reported in ppm relative to deuterochloroform $(77.16 \mathrm{ppm})$, unless otherwise stated. All crude NMR analysis were performed by using trifluorotoluene as the internal standard. High resolution mass spectrometry (HRMS) was obtained using the APCI and ESI technique. Mass spectrometry (MS) was recorded on a Shimadzu GC-MS-QP2020. Optical rotation was measured on an AUTOPOL IV polarimeter.

Chiral SFC analysis/Chiral HPLC analysis: Chiral SFC and HPLC were performed using Chiralpak IF, Chiralpak IB, Chiralpak IA, Chiralcel OJ-H, Chiralpak IC, Chiralpal AS-H, and Chiralpak OZ-H columns $(4.6 \times 250 \mathrm{~mm} \times 5 \mu \mathrm{m})$ eluting with $\mathrm{MeOH} / \mathrm{CO}_{2}(\mathrm{SFC})$ or $\mathrm{PrOH} / \mathrm{hexane}$ (HPLC) and monitored by DAD (Diode Array Detector). Retentions times $\left(t_{\mathrm{R}}\right)$ are quoted in minutes.

Chiral GC analysis: Chiral GC was performed using a Chiraldex $\beta$-6TBDM 30 m column, Helium gas carrier at $1 \mathrm{~mL} / \mathrm{min}$, constant pressure, with a MS detector. Retentions times $\left(t_{\mathrm{R}}\right)$ are quoted in minutes. 


\section{Protection of allylboronic acids for isolation}

Allylboronic acids (such as 1a) are oxygen sensitive species, and therefore their purification and isolation require protection of the $\mathrm{B}(\mathrm{OH})_{2}$ functionality. The protection of the $\mathrm{B}(\mathrm{OH})_{2}$ group can be carried out smoothly using the crude reaction mixture of the homologation using diamine and dialcohol species (Figure S1). We have found diamino naphthalene (Dan) protected 5a the most stable species. After purification with column chromatography the Dan protected allylboronic acids can be hydrolysed under acidic conditions affording purified allylboronic acids in high yield. Using pinacol instead of DanH the corresponding pinacol borane 1a-Bpin can be obtained in 65\% yield (based on vinyl- $\mathrm{B}(\mathrm{OH})_{2}$ ). Alternatively, $(+)$-pinanediol can be added affording 1a-Bpinane in 73\% yield. Neither 1a-Bpin nor 1a-Bpinane could be hydrolyzed to allylboronic acid 1a. Therefore, these compounds cannot be used, as synthetic intermediates for purification (and thus recovery) of allylboronic acids $\mathbf{1}$. In addition, 1a-Bpin and 1a-Bpinane are much less reactive than the corresponding allylboronic acid 1a. For example, 1a reacted readily with ketones and imines without any additive with high level of chirality transfer (Table 2 in the main text), while 1a-Bpin and 1a-Bpinane are completely inert under these reactions. Considering the above, we used Dan protection of the allylboronic acids for purification and determination of the enantioselelectivity of the homologation reactions.

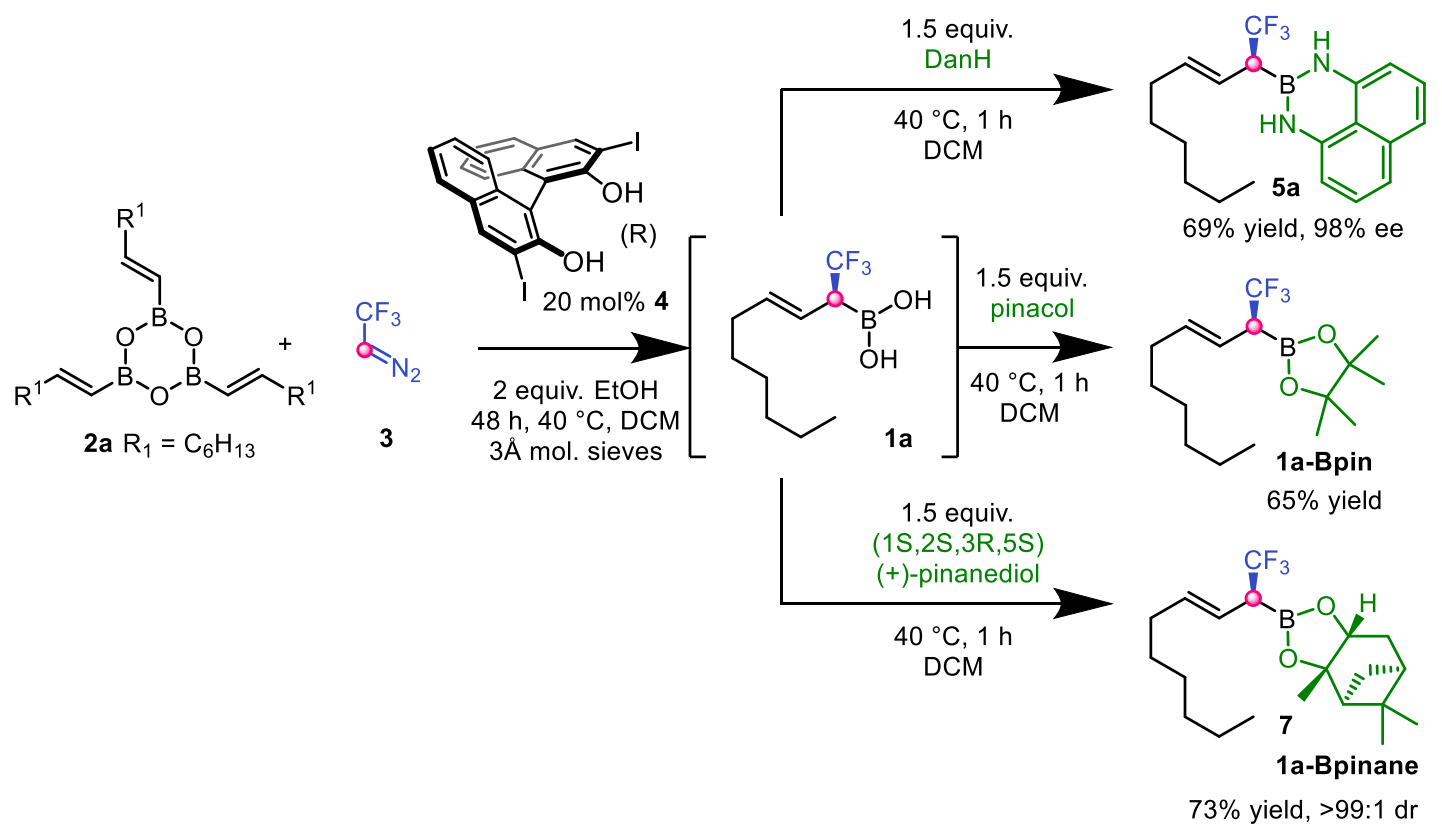

Figure S1. Protection possibilities of allylboronic acids. 


\section{Experimental Procedures and Spectral data}

\subsection{Preparation of the alkenylboroxines}

The aliphatic alkenylboronic acids 2 a-c $(0.6 \mathrm{mmol})$ were dissolved in $7 \mathrm{~mL}$ of dry DCM and stored overnight over activated $3 \AA$ molecular sieves. The DCM solution was transferred into a round-bottom flask and the solvent was removed under vacuum. The boroxine obtained was dissolved in dry DCM to make a $0.1 \mathrm{mmol} / \mathrm{mL}$ stock solution of the boroxine. The aromatic alkenylboronic acids 2d-g were dissolved in toluene and refluxed in a Dean Stark apparatus to obtain their respective boroxine.

The ${ }^{1} \mathrm{H}$ NMR and ${ }^{11} \mathrm{~B}$ NMR spectra of boroxine 2a compared to the commercially available boronic acid (as received) is given bellow. The ${ }^{1} \mathrm{H}$ NMR spectrum of commercially available boronic acid shows a ratio of 1:1 for the vinylic proton of the boroxine $(5.72 \mathrm{ppm})$ and that of the boronic acid (5.26 ppm). After drying the ${ }^{1} \mathrm{H}$ NMR spectrum of boroxine 2a shows a ratio of 100:7, indicating almost complete conversion to the boroxine. The ${ }^{1} \mathrm{H}$ NMR and ${ }^{11} \mathrm{~B}$ NMR spectra of all boroxines 2a-h are given in the NMR spectra section of this document.

${ }^{1} \mathrm{H}$ NMR (Toluene- $d 8,400 \mathrm{MHz}$ ) of the boronic acid as received (a) and coverted to the boroxine 2a (b).
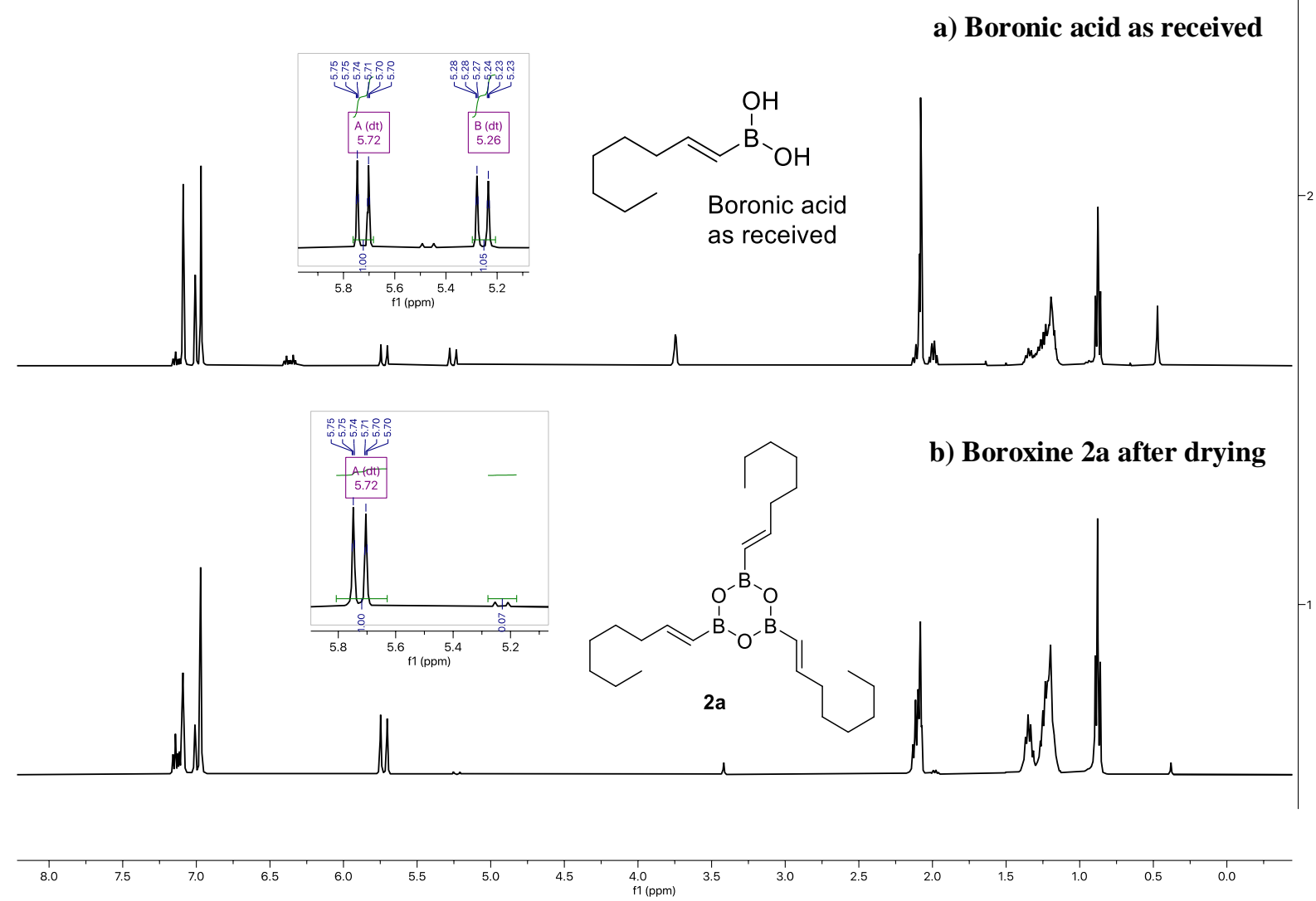
${ }^{11} \mathrm{~B}$ NMR (Toluene- $d 8,128 \mathrm{MHz}$ ) of the boronic acid as received (a) and converted to the boroxine (b).

a) Boronic acid as received
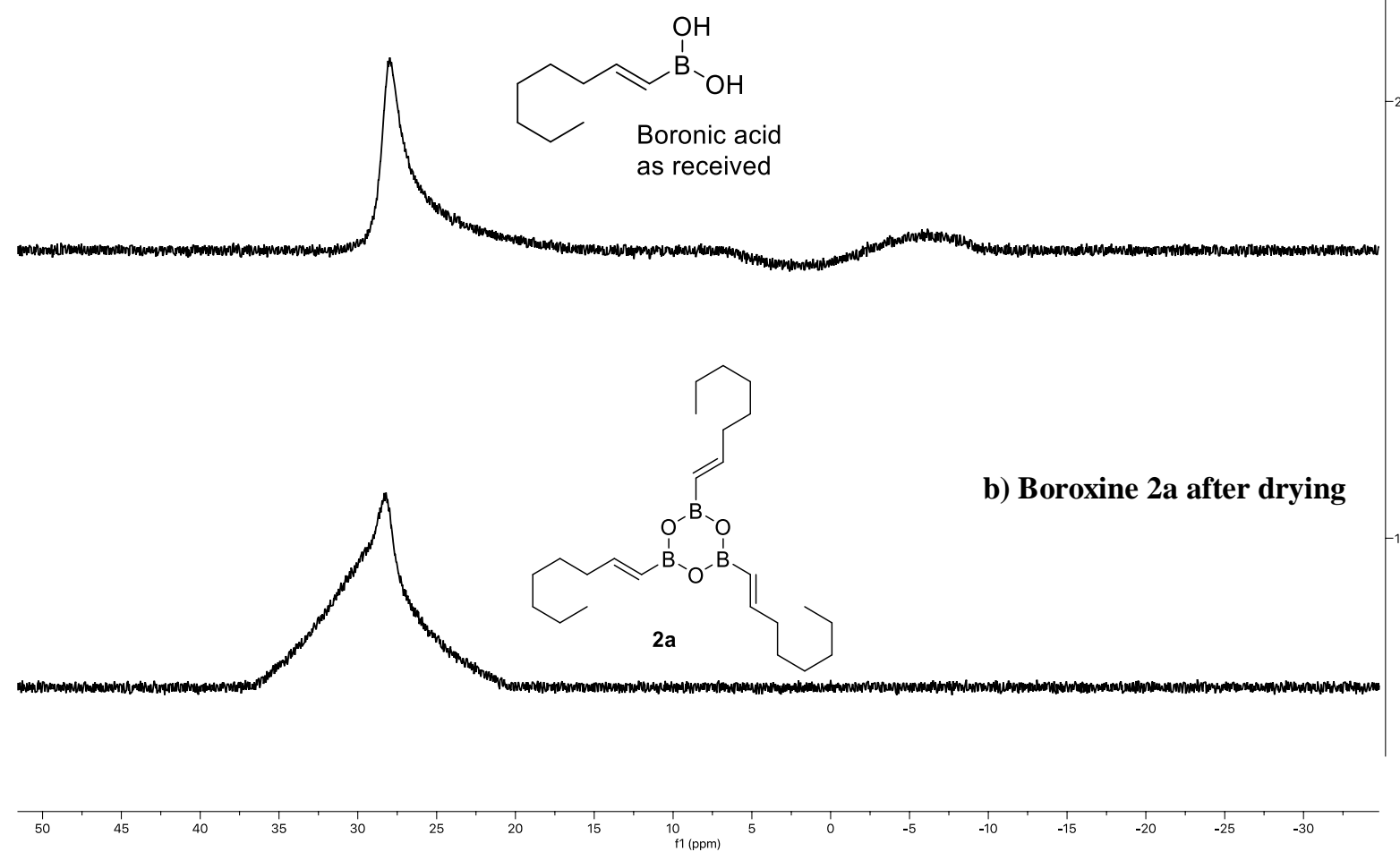

2,4,6-tri((E)-oct-1-en-1-yl)-1,3,5,2,4,6-trioxatriborinane (2a): Boroxine 2a was obtained as a

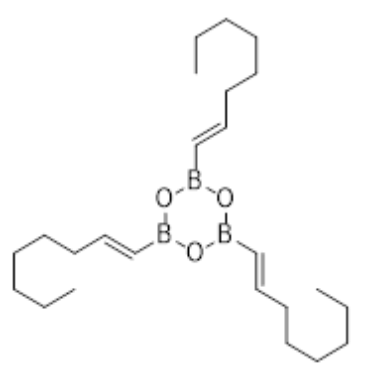
colorless oil by the above general procedure (ratio of boroxine/boronic acid is 100:7). ${ }^{1} \mathbf{H}$ NMR (400 MHz, Toluene-d8) $\boldsymbol{\delta} 7.17-7.10(\mathrm{~m}, 1 \mathrm{H}$, overlapping), $5.72(\mathrm{dd}, J=17.6,1.6 \mathrm{~Hz}, 1 \mathrm{H}), 2.16-2.05(\mathrm{~m}, 2 \mathrm{H}), 1.43-$ $1.30(\mathrm{~m}, 2 \mathrm{H}), 1.29-1.14(\mathrm{~m}, 6 \mathrm{H}), 0.88(\mathrm{t}, J=6.9 \mathrm{~Hz}, 3 \mathrm{H}) ;{ }^{11} \mathbf{B}$ NMR (128 MHz, Toluene-d8) $\delta$ 28.32. 
2,4,6-tri((E)-prop-1-en-1-yl)-1,3,5,2,4,6-trioxatriborinane (2b): Boroxine $2 \mathrm{~b}$ was obtained as a white solid by the above general procedure (100\% boroxine). ${ }^{1} \mathbf{H}$ NMR (400 MHz, Toluene-d8) $\delta 7.06-6.95(\mathrm{~m}, 1 \mathrm{H}$, overlapping), $5.65(\mathrm{dd}, J=17.5,1.7$ $\mathrm{Hz}, 1 \mathrm{H}), 1.69$ (dd, $J=6.5,1.7 \mathrm{~Hz}, 3 \mathrm{H}) ;{ }^{11}$ B NMR (128 MHz, Toluene-d8) $\delta$ 27.93.

2,4,6-tris((E)-3-phenylprop-1-en-1-yl)-1,3,5,2,4,6-trioxatriborinane (2c): Boroxine 2c was

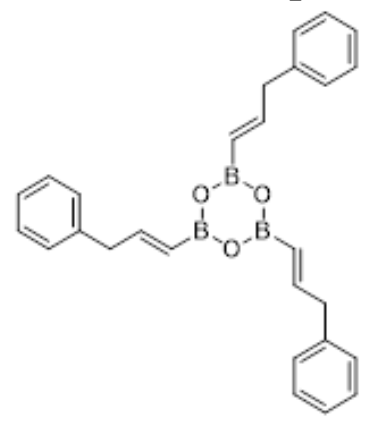
obtained as a pale brown oil by the above general procedure(ratio of boroxine/boronic acid is 100:5). ${ }^{\mathbf{1}} \mathrm{H}$ NMR (400 MHz, Toluene-d8) $\boldsymbol{\delta} 7.15$ $-6.92(\mathrm{~m}, 6 \mathrm{H}$, overlapping), 5.59 (dt, $J=17.6,1.6 \mathrm{~Hz}, 1 \mathrm{H}), 3.27$ (dd, $J=$ 6.5, 1.6 Hz, 2H); ${ }^{11}$ B NMR (128 MHz, Toluene-d8) $\delta$ 28.22.

2,4,6-tri(E-styryl)-1,3,5,2,4,6-trioxatriborinane (2d): Boroxine 2d was obtained as a white solid

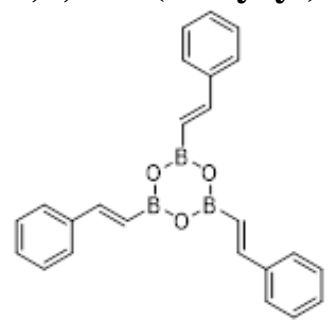
by the above general procedure (ratio of boroxine/boronic acid is 100:8). ${ }^{1} \mathbf{H}$ NMR (400 MHz, Toluene-d8) $\delta 7.91(\mathrm{~d}, J=18.1 \mathrm{~Hz}, 1 \mathrm{H}), 7.48-7.32(\mathrm{~m}$, 2H), $7.19-7.05\left(\mathrm{~m}, 6 \mathrm{H}\right.$, overlapping), $6.43(\mathrm{~d}, J=18.1 \mathrm{~Hz}, 1 \mathrm{H}) ;{ }^{11} \mathbf{B}$ NMR (128 MHz, Toluene-d8) $\delta 32.34$.

2,4,6-tris(E-4-bromostyryl)-1,3,5,2,4,6-trioxatriborinane (2e): Boroxine 2e was obtained as a

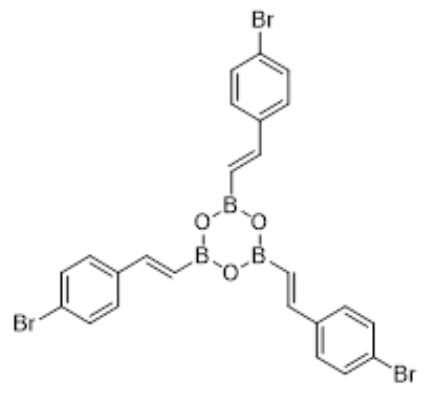
white solid by the above general procedure (ratio of boroxine/boronic acid is 100:11). ${ }^{\mathbf{1}} \mathbf{H}$ NMR (400 MHz, Toluene-d8) $\delta 7.71(\mathrm{~d}, J=18.1$ $\mathrm{Hz}, 1 \mathrm{H}), 7.18$ (d, $J=8.4 \mathrm{~Hz}, 2 \mathrm{H}), 7.03$ (d, $J=8.9 \mathrm{~Hz}, 2 \mathrm{H}), 6.30$ (d, $J=$ $18.0 \mathrm{~Hz}, 1 \mathrm{H}) ;{ }^{11}$ B NMR (128 MHz, Toluene-d8) $\delta$ 28.26. 
2,4,6-tris(E-4-chlorostyryl)-1,3,5,2,4,6-trioxatriborinane (2f): Boroxine $2 \mathrm{f}$ was obtained as a

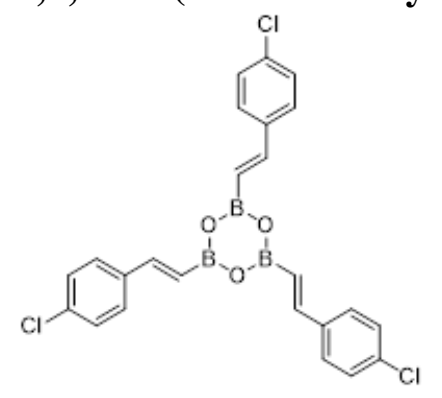

white solid by the above general procedure. (ratio of boroxine/boronic acid is 100:9). ${ }^{\mathbf{1}} \mathbf{H}$ NMR (400 MHz, Toluene-d8) $\boldsymbol{\delta} 7.74(\mathrm{~d}, J=18.1$ $\mathrm{Hz}, 1 \mathrm{H}), 7.11(\mathrm{~d}, J=8.6 \mathrm{~Hz}, 2 \mathrm{H}), 7.03(\mathrm{~d}, J=8.6 \mathrm{~Hz}, 2 \mathrm{H}), 6.29(\mathrm{~d}, J=$ 18.1 Hz, 1H); ${ }^{11}$ B NMR (128 MHz, Toluene-d8) $\delta$ 28.22.

2,4,6-tris((E)-4-(trifluoromethyl)styryl)-1,3,5,2,4,6-trioxatriborinane (2g): Boroxine $2 \mathrm{~g}$ was

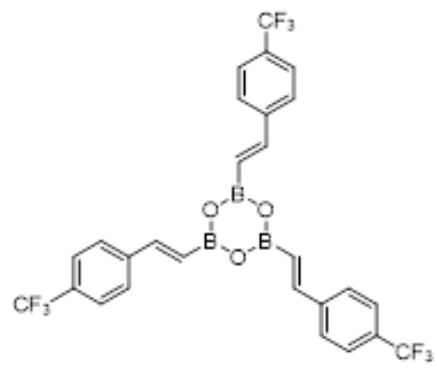
obtained as a white solid by the above general procedure (ratio of boroxine/boronic acid is 100:6). ${ }^{1} \mathrm{H}$ NMR (400 MHz, Toluene-d8) $\delta$ $7.77(\mathrm{~d}, J=18.1 \mathrm{~Hz}, 1 \mathrm{H}), 7.30(\mathrm{~d}, J=8.1 \mathrm{~Hz}, 2 \mathrm{H}), 7.21(\mathrm{~d}, J=8.1 \mathrm{~Hz}$, 2H), 6.39 (d, $J=18.1 \mathrm{~Hz}, 1 \mathrm{H}) ;{ }^{11}$ B NMR (128 MHz, Toluene-d8) $\delta$ 28.39 .

2,4,6-tris((E)-4-fluorostyryl)-1,3,5,2,4,6-trioxatriborinane (2h): Boroxine $2 \mathrm{~h}$ was obtained as a

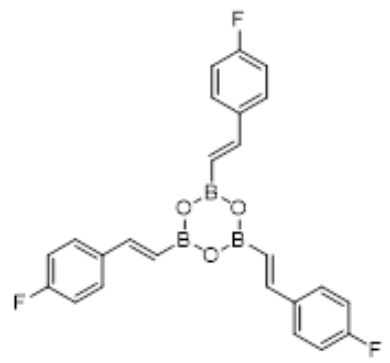
white solid by the above general procedure (ratio of boroxine/boronic acid is 100:9). ${ }^{\mathbf{1}} \mathbf{H}$ NMR (400 MHz, Toluene-d8) $\boldsymbol{\delta} 7.78(\mathrm{~d}, J=18.1 \mathrm{~Hz}$, $1 \mathrm{H}), 7.18(\mathrm{dd}, J=8.6,5.5 \mathrm{~Hz}, 2 \mathrm{H}), 6.72(\mathrm{t}, J=8.6 \mathrm{~Hz}, 2 \mathrm{H}), 6.26(\mathrm{~d}, J=$ 18.1 Hz, 1H); ${ }^{11}$ B NMR (128 MHz, Toluene-d8) $\delta 28.04$.

\subsection{General procedure A: Asymmetric homologation of vinyl boronic acid}

An oven-dried screw cap reaction tube was charged with (R)-iodo-BINOL 4 (0.02 mmol). The reaction tube was brought into the glovebox and vinyl boroxine $2(0.033 \mathrm{mmol}$ corresponding to $0.1 \mathrm{mmol}$ vinylboronic acid) in DCM $(0.33 \mathrm{~mL})$, ethanol $(0.2 \mathrm{mmol})$, molecular sieves $(20 \mathrm{mg})$, $\mathrm{CF}_{3}$-diazomethane $3(0.3 \mathrm{mmol})$ in DCM $(0.4 \mathrm{~mL})$ were added sequentially. The total volume was maintained to $0.8 \mathrm{~mL}$. The reaction tube was tightly screwed and brought out to the fumehood. The reaction mixture was stirred at $40{ }^{\circ} \mathrm{C}$ for 48 hours. After 48 hours, DanH (0.15 mmol) was added inside glovebox and stirred for another hour. NMR of the crude reaction mixture was recorded using trifluorotoluene as an internal standard. The product was isolated by silica with a 
suitable solvent, either pentane/ethyl acetate or pentane/DCM system. The product was visualized using UV fluorescence and/or developed with permanganate stain.

Preparation of racemate for SFC/HPLC/GC: The racemates of allyl Bdan products were prepared by following the above procedure except that the equimolar mixture of (R) and (S) 3,3'dibromo-BINOL was used as a catalyst. Minor deviations of a 50/50 ratio of the S/R enantiomers may be due to weighing errors.

\subsection{General procedure B: Asymmetric homologation of vinyl boronic acid using TMS diazocompound}

An oven-dried screw cap reaction tube was charged with (R)-3,3'-diiodo-[1,1'binaphthalene]-2,2'-diol 4 (0.02 mmol) and boroxin of (E)-(4-fluorostyryl)boronic acid $\mathbf{2 h}(0.1$ $\mathrm{mmoL})$. The reaction tube was brought into the glovebox and dry toluene $(0.6 \mathrm{~mL})$, ethanol $(0.2$ $\mathrm{mmoL})$, molecular sieves $(20 \mathrm{mg})$ TMS-diazomethane $\left(0.3 \mathrm{mmoL}, 2.0 \mathrm{M}\right.$ solution in $\left.\mathrm{Et}_{2} \mathrm{O}\right)$ were added sequentially. The reaction tube was tightly screwed and brought out to the hood. The reaction mixture was stirred at $60{ }^{\circ} \mathrm{C}$ for 3 hours. After 3 hours, (1S,2S,3R,5S)-(+)-pinanediol $(0.15 \mathrm{mmol})$ was inside glovebox added and stirred for another hour. The product was isolated on silica using 40:1 pentane/ethyl acetate as eluent. The product was visualized using UV fluorescence.

\subsection{General Procedure C: One-pot allylboration of aldehyde 6a}

The general procedure A was used except that after $48 \mathrm{~h}$, the actual volume is reduced to $0.5 \mathrm{~mL}$ by Ar flow to remove the unreacted (excess) of $\mathbf{3}$ (Note). Subsequently, aldehyde $\mathbf{6 a}(0.15$ mmol) was added instead of DanH. Then, the reaction mixture was stirred at $40{ }^{\circ} \mathrm{C}$ for 4 hours. The homoallylic alcohol product was isolated by silica gel chromatography. In these reactions a single diastereomer of the homoallylic product was formed, in which the double bond geometry was $E$.

Note: The reason for reducing the reaction volume to $0.5 \mathrm{~mL}$ in the one-pot allylboration reaction was to remove the unreacted $\mathrm{CF}_{3}$-diazomethane (3). Compound $\mathbf{3}$ is volatile, and therefore it was applied in excess for the homologation step. However, $\mathbf{3}$ may react with aldehydes (or other electrophiles), and therefore it had to be removed prior to the allylboration step. The reproducibility of the reduction of the amount of solvent was assured by pre-calibrating the vial with $0.5 \mathrm{~mL}$ solvent and marking it. The Ar flow was applied until the solvent level decreased to the pre-calibrated mark.

\subsection{General Procedure D: In situ oxidation of allylboronic acids}

The general procedure A was used except that after $48 \mathrm{~h}$, the DCM was removed by $\mathrm{Ar}$ flow and toluene $(1 \mathrm{~mL})$ was added. Subsequently, $3 \mathrm{~N} \mathrm{NaOH}(0.3 \mathrm{mmol}, 0.1 \mathrm{~mL})$ and $\mathrm{H}_{2} \mathrm{O}_{2}(0.3$ mmol, $0.1 \mathrm{~mL}, 35 \mathrm{wt}$. $\%$ in $\mathrm{H}_{2} \mathrm{O}$ ) were added under $0{ }^{\circ} \mathrm{C}$. Then, the reaction mixture was stirred at room temperature for overnight. The reaction mixture was acidified with $1 \mathrm{M} \mathrm{HCl}$ and $3 \mathrm{~mL}$ water 
was added. The organic layer was extracted with diethyl ether and the aqueous layer was washed 3 times with diethyl ether. The combined organic phase was passed through phase separator and the solvent was evaporated under reduced pressure. The chiral allyl alcohol was isolated by silica gel chromatography.

\subsection{General procedure E: Hydrolysis of BDan and allylboration reaction with isolated allylboronic acid}

The corresponding allyl-Bdan $\mathbf{5 a} / \mathbf{5} \mathbf{d}(0.1 \mathrm{mmoL})$ was taken in a screw capped reaction tube. $600 \mu \mathrm{L}$ degassed DME was added followed by $500 \mu \mathrm{L} 3 \mathrm{~N} \mathrm{H}_{2} \mathrm{SO}_{4}$ under argon. The mixture was stirred overnight at room temperature. Then, $0.5 \mathrm{M} \mathrm{HCl}$ was added as well as toluene and shaken vigorously. The toluene layer was passed through a phase separator in glovebox and dried over molecular sieves. Then, the electrophile $6(0.15 \mathrm{mmol})$ was added and stirred for overnight at room temperature. (NMR spectra of purified allylboronic acid 1a and 1d are given below in page S41-47)

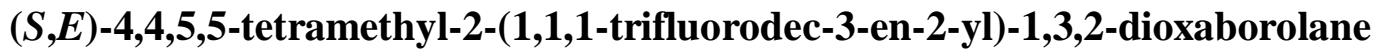

(1a-Bpin):

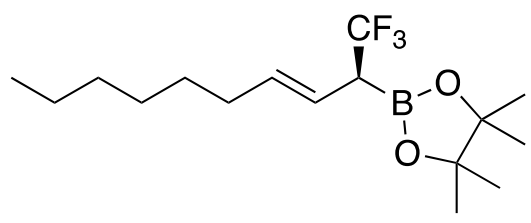

This compound was obtained according to the above general procedure A except that after $48 \mathrm{~h}$, pinacol $(0.15 \mathrm{mmol})$ was used. Product 1a-Bpin was obtained by silica gel chromatography using pentane/ethyl acetate 100:1 as eluent. The oily product was dissolved in pentane and traces of catalyst 4 were removed by filtration over cotton wool. Product 1a-Bpin was isolated in 65\% yield (20.8 $\mathrm{mg}, 65 \mu \mathrm{mol})$ as a colorless oil.

${ }^{1} \mathbf{H}$ NMR (400 MHz, CDCl 3 ) $\boldsymbol{\delta} 5.65$ (dt, $\left.J=15.3,6.8 \mathrm{~Hz}, 1 \mathrm{H}\right), 5.39$ (ddt, $J=15.4,8.9,1.5 \mathrm{~Hz}$, $1 \mathrm{H}), 2.65(\mathrm{qd}, J=11.2,8.7 \mathrm{~Hz}, 1 \mathrm{H}), 2.16-1.88(\mathrm{~m}, 2 \mathrm{H}), 1.42-1.32(\mathrm{~m}, 1 \mathrm{H}), 1.26(\mathrm{~d}, J=4.9 \mathrm{~Hz}$, $16 \mathrm{H}), 1.00-0.72(\mathrm{~m}, 2 \mathrm{H}) ;{ }^{13} \mathbf{C} \mathbf{N M R}\left(\mathbf{1 0 1} \mathbf{M H z}, \mathbf{C D C l}_{3}\right) \boldsymbol{\delta} 137.14,127.16\left(\mathrm{q},{ }^{1} J_{C-F}=277.4 \mathrm{~Hz}\right)$, $119.38\left(\mathrm{q},{ }^{3} J_{C-F}=3.9 \mathrm{~Hz}\right), 84.44,32.78,31.81,29.12,28.78,24.75,24.50,22.78,14.22 ;{ }^{19} \mathbf{F ~ N M R}$ $\left(377 \mathbf{M H z}, \mathbf{C D C l}_{3}\right) \boldsymbol{\delta}-63.58(\mathrm{~d}, J=11.2 \mathrm{~Hz}) ;{ }^{11} \mathbf{B}$ NMR (128 $\left.\mathbf{M H z}, \mathbf{C D C l}_{3}\right) \delta$ 31.10; HRMS (pos. ESI) $\mathbf{~ m} / \mathbf{z}$ : calcd for $\mathrm{C}_{16} \mathrm{H}_{28} \mathrm{BF}_{3} \mathrm{NaO}_{2}[\mathrm{M}+\mathrm{Na}]^{+}$343.2030. Found 343.2036; $[\boldsymbol{\alpha}]_{\boldsymbol{D}}^{\mathbf{2 4}}=-20.8(c$ $\left.0.52, \mathrm{CHCl}_{3}\right)$.

(3aS,4S,6S,7aR)-3a,5,5-trimethyl-2-((S,E)-1,1,1-trifluorodec-3-en-2-yl)hexahydro-4,6methanobenzo[d][1,3,2] dioxaborole (1a-Bpinane): This compound was obtained according to

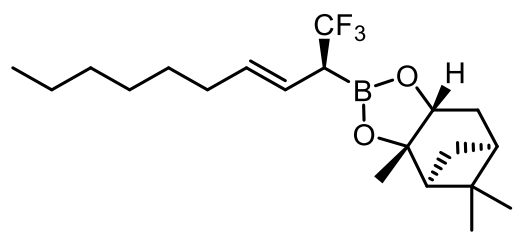

the above general procedure A except that after $48 \mathrm{~h}$, pinanediol $(0.15 \mathrm{mmol})$ was used. Product 1a-Bpinane was isolated in $73 \%$ yield $(27.3 \mathrm{mg}, 73 \mu \mathrm{mol})$ as a colorless oil by silica gel chromatography using pentane/ethyl acetate 100:1 as eluent.

${ }^{1} \mathbf{H}$ NMR (400 MHz, CDCl 3 ) $\delta 5.67(\mathrm{dt}, J=14.2,6.8 \mathrm{~Hz}, 1 \mathrm{H})$, $5.42(\mathrm{dd}, J=15.4,9.0 \mathrm{~Hz}, 1 \mathrm{H}), 4.34(\mathrm{dd}, J=8.8,2.0 \mathrm{~Hz}, 1 \mathrm{H})$, 2.71 (qd, $J=11.2,8.8 \mathrm{~Hz}, 1 \mathrm{H}), 2.35$ (ddt, $J=14.0,8.9,2.4 \mathrm{~Hz}, 1 \mathrm{H}), 2.23(\mathrm{dtd}, J=11.3,6.1,2.3$ $\mathrm{Hz}, 1 \mathrm{H}), 2.12-1.99(\mathrm{~m}, 3 \mathrm{H}), 1.96-1.89$ (m, 1H), 1.86 (ddd, $J=14.5,3.4,2.1 \mathrm{~Hz}, 1 \mathrm{H}), 1.40$ (s, $3 \mathrm{H}), 1.37-1.31(\mathrm{~m}, 2 \mathrm{H}), 1.29(\mathrm{~s}, 3 \mathrm{H}), 1.28-1.24(\mathrm{~m}, 5 \mathrm{H}), 1.09(\mathrm{~d}, J=11.0 \mathrm{~Hz}, 1 \mathrm{H}), 0.88(\mathrm{td}, J$ $=7.0,2.7 \mathrm{~Hz}, 4 \mathrm{H}), 0.84(\mathrm{~s}, 3 \mathrm{H}) ;{ }^{13} \mathbf{C}$ NMR (101 MHz, $\left.\mathbf{C D C l}_{3}\right) \boldsymbol{\delta} 137.19,127.17\left(\mathrm{q},{ }^{1} J_{C-F}=277.4\right.$ $\mathrm{Hz}), 119.52\left(\mathrm{q},{ }^{3} J_{C-F}=3.8 \mathrm{~Hz}\right), 86.83,78.54,51.26,39.51,38.31,35.42,34.28,32.78,31.82$, 29.11, 28.79, 28.53, 27.15, 26.45, 24.12, 22.77, 22.50, 14.22.; ${ }^{19} \mathbf{F}$ NMR (377 MHz, $\left.\mathbf{C D C l}_{3}\right) \boldsymbol{\delta}$ 
-63.58 (d, $J=11.2 \mathrm{~Hz})$; ${ }^{11} \mathbf{B}$ NMR (128 $\left.\mathbf{M H z}, \mathbf{C D C l}_{3}\right) \boldsymbol{\delta} 30.08 ; \mathbf{H R M S}$ (pos. ESI) m/z: calcd for $\mathrm{C}_{20} \mathrm{H}_{32} \mathrm{BF}_{3} \mathrm{NaO}_{2}[\mathrm{M}+\mathrm{Na}]^{+}$395.2343. Found 395.2354; $[\boldsymbol{\alpha}]_{\boldsymbol{D}}^{26}=-6.2\left(c\right.$ 0.55, $\left.\mathrm{CHCl}_{3}\right)$. 
(S,E)-2-(1,1,1-Trifluorodec-3-en-2-yl)-2,3-dihydro-1H-naphtho[1,8-de][1,3,2]diazaborinine (5a): This compound was obtained according to the above general procedure A. Product 5a was<smiles>CCCCCCC=CC(B1Nc2cccc3cccc(c23)N1)C(F)(F)F</smiles>
isolated in $69 \%$ yield $(24.7 \mathrm{mg}, 69 \mu \mathrm{mol})$ as light yellow oil by silica gel chromatography using petroleum ether/ethyl acetate $20: 1$ as eluent $(0.1 \mathrm{mmol}$ scale reaction). Product 5a was isolated in $78 \%$ yield $(281.5 \mathrm{mg}, 0.78 \mathrm{mmol})$ as light yellow oil by silica gel chromatography using pentane:DCM $4: 1$ as eluent ( $1 \mathrm{mmol}$ scale reaction).

${ }^{1}$ H NMR (400 MHz, CDCl $)$ ) $7.17-6.97(\mathrm{~m}, 4 \mathrm{H}), 6.34(\mathrm{dd}, J=7.2,1.2 \mathrm{~Hz}, 2 \mathrm{H}), 5.80-5.65$ (m, 3H), 5.47 (ddt, $J=15.4,9.3,1.5 \mathrm{~Hz}, 1 \mathrm{H}), 2.68(\mathrm{qd}, J=11.8,9.1 \mathrm{~Hz}, 1 \mathrm{H}), 2.26-1.95(\mathrm{~m}, 2 \mathrm{H})$, $1.42(\mathrm{q}, J=7.2 \mathrm{~Hz}, 2 \mathrm{H}), 1.37-1.21(\mathrm{~m}, 6 \mathrm{H}), 1.05-0.44(\mathrm{~m}, 3 \mathrm{H}) .{ }^{\mathbf{1 3}} \mathbf{C} \mathbf{N M R}\left(\mathbf{1 0 1} \mathbf{~ M H z}, \mathbf{C D C l}_{\mathbf{3}}\right)$ $\boldsymbol{\delta} 140.29,137.68,136.34,132.07,129.31,127.68,126.55,120.63\left(\mathrm{q},{ }^{3} J_{C-F}=3.7 \mathrm{~Hz}\right), 119.92$, $118.44,106.43,32.85,31.81,29.26,28.95,22.77,14.22 ;{ }^{19} \mathbf{F}$ NMR (377 $\left.\mathbf{~ M H z}, \mathbf{C D C l}_{3}\right) \boldsymbol{\delta}-62.39$ $(\mathrm{d}, J=11.7 \mathrm{~Hz}) ;{ }^{11} \mathbf{B}$ NMR (128 MHz, $\left.\mathbf{C D C l}_{3}\right) \delta$ 29.47; HRMS (neg. ESI) m/z: calcd for $\mathrm{C}_{20} \mathrm{H}_{24} \mathrm{BClF}_{3} \mathrm{~N}_{2}[\mathrm{M}+\mathrm{Cl}]^{-}$395.1682. Found 395.1701. $[\boldsymbol{\alpha}]_{\boldsymbol{D}}^{27}=-48.9\left(c 0.35, \mathrm{CHCl}_{3}\right)$.

Determination of $\boldsymbol{e} e$ by Chiral SFC: Diacel CHIRALPAK IB N-3, $25^{\circ} \mathrm{C}, 0.3 \mathrm{~cm} \phi, 15 \mathrm{~cm}$ column, $10 \% \mathrm{MeOH}$ in $\mathrm{CO}_{2}$, flow rate: $0.8 \mathrm{~mL} / \mathrm{min}$; $t \mathrm{R}: 6.48 \mathrm{~min}$ (minor enantiomer), $7.98 \mathrm{~min}$ (major enantiomer); ee (major enantiomer) $=98 \%$.

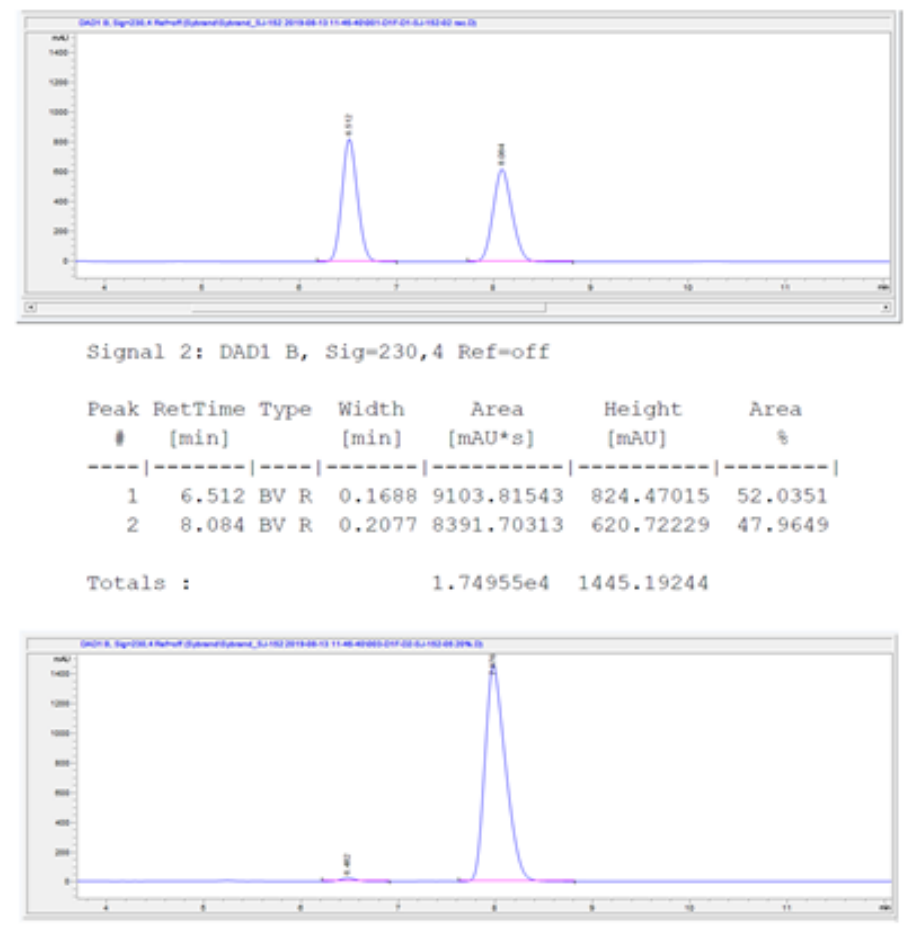

Signal 2: DAD1 B, Sig $=230,4$ Ref $=0 f f$

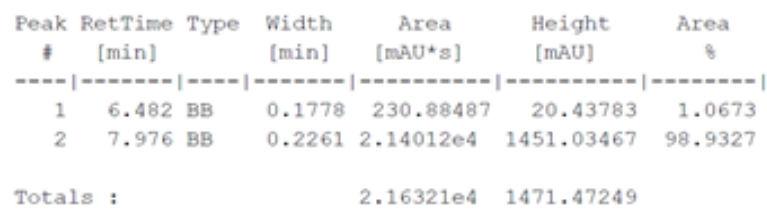


Reaction on 2 mmol scale: An oven-dried $25 \mathrm{~mL}$ screw cap reaction tube was charged with (R)iodo-BINOL $4(0.4 \mathrm{mmol})$. The reaction tube was brought into the glovebox and vinyl boroxine 2 (0.66 mmol corresponding to $2 \mathrm{mmol}$ vinylboronic acid) in DCM (6.66 mL), ethanol (4 mmol), molecular sieves (400 mg), $\mathrm{CF}_{3}$-diazomethane $3(2 \mathrm{mmol})$ in DCM $(6 \mathrm{~mL})$ were added sequentially. The total volume was maintained to $14 \mathrm{~mL}$. The reaction tube was tightly screwed and brought out to the fumehood. The reaction mixture was stirred at $40{ }^{\circ} \mathrm{C}$ for 24 hours. After 24 hours, another aliquot of $\mathrm{CF}_{3}$-diazomethane $3(1 \mathrm{mmol})$ in DCM $(3 \mathrm{~mL})$ was added. The reaction mixture was stirred at $40{ }^{\circ} \mathrm{C}$ for 48 hours. After 72 hours total reaction time, DanH ( 3 mmol) was added inside glovebox and stirred for another hour. Product 5a was isolated in 68\% yield (490.0 $\mathrm{mg}, 1.36 \mathrm{mmol}$ ) as light yellow oil by silica gel chromatography using pentane:DCM 4:1 as eluent.

Determination of $\boldsymbol{e} e$ by Chiral SFC ( 2 mmol scale reaction): Diacel CHIRALPAK IB N-3, 25 ${ }^{\circ} \mathrm{C}, 0.3 \mathrm{~cm} \phi, 15 \mathrm{~cm}$ column, $10 \% \mathrm{MeOH}$ in $\mathrm{CO}_{2}$, flow rate: $0.8 \mathrm{~mL} / \mathrm{min}$; $t \mathrm{R}: 7.07 \mathrm{~min}$ (minor enantiomer), $8.52 \mathrm{~min}$ (major enantiomer); ee (major enantiomer) $=96 \%$.

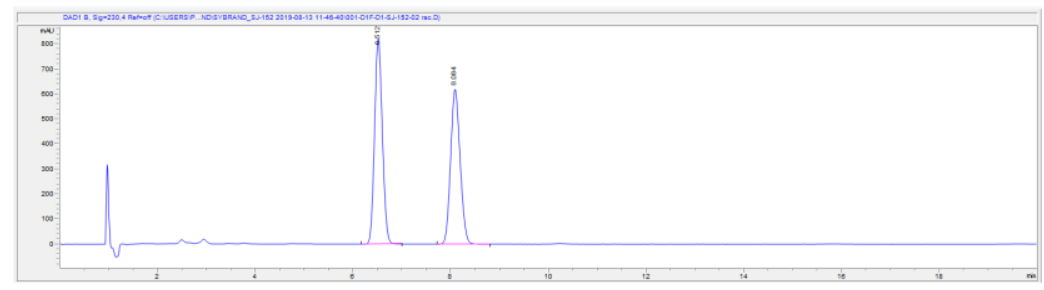

Signal 2: DAD1 B, Sig-230,4 Ref-off

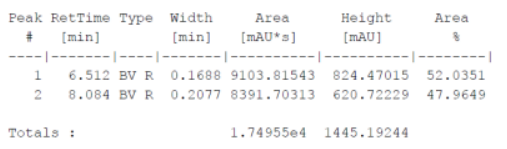

Totals : $\quad 1.74955 \mathrm{e} 4 \quad 1445.19244$

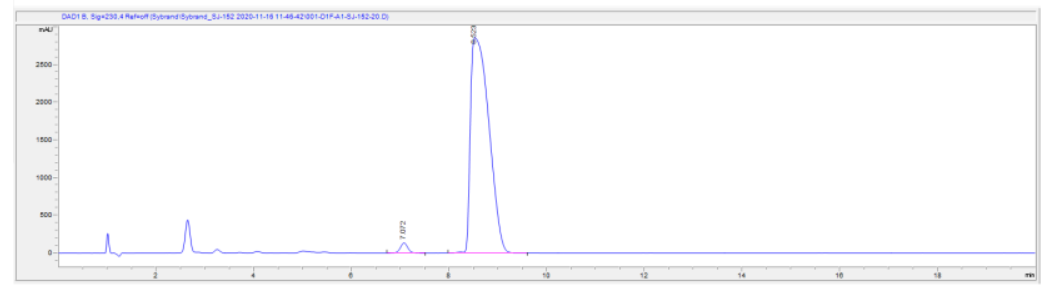

Signal 2: DAD1 B, Sig=230,4 Ref=off

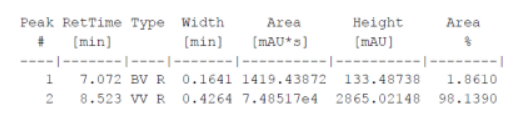

Totals : $\quad 7.62711 \mathrm{e} \quad 2998.50887$ 
(S,E)-2-(1,1,1-Trifluoropent-3-en-2-yl)-2,3-dihydro-1H-naphtho[1,8-de][1,3,2]diazaborinine<smiles>C/C=C/C(B1Nc2cccc3cccc(c23)N1)C(F)(F)F</smiles>
(5b): This compound was obtained according to the above general procedure A. Product $\mathbf{5 b}$ was isolated in $48 \%$ yield $(13.8 \mathrm{mg}, 48 \mu \mathrm{mol})$ as light yellow oil by silica gel chromatography using petroleum ether/ethyl acetate $20: 1$ as eluent.

${ }^{1}$ H NMR (400 MHz, $\left.\mathbf{C D C l}_{3}\right) \boldsymbol{\delta} 7.18-6.99(\mathrm{~m}, 4 \mathrm{H}), 6.35(\mathrm{dd}, J=7.2,1.1$ $\mathrm{Hz}, 2 \mathrm{H}), 5.85-5.66(\mathrm{~m}, 3 \mathrm{H}), 5.49$ (ddq, $J=15.3,9.2,1.7 \mathrm{~Hz}, 1 \mathrm{H}), 2.68$ (qd, $J=11.7,9.1 \mathrm{~Hz}, 1 \mathrm{H}), 1.80(\mathrm{dd}, J=6.5,1.7 \mathrm{~Hz}, 3 \mathrm{H}) ;{ }^{13} \mathbf{C}$ NMR $(\mathbf{1 0 1} \mathbf{M H z}$, Chloroform-d)

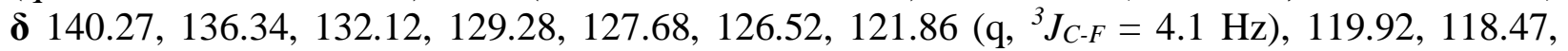
106.45, 18.36; ${ }^{19} \mathbf{F}$ NMR $\left(377 \mathbf{~ M H z}, \mathbf{C D C l}_{3}\right) \delta-62.44(\mathrm{~d}, J=11.7 \mathrm{~Hz}) ;{ }^{11} \mathbf{B}$ NMR (160 MHz, $\left.\mathbf{C D C l}_{3}\right) \delta$ 28.78; HRMS (pos. APCI) $\mathbf{m} / \mathbf{z}$ : calcd for $\mathrm{C}_{15} \mathrm{H}_{15} \mathrm{BF}_{3} \mathrm{~N}_{2}[\mathrm{M}+\mathrm{H}]^{+}$291.1278. Found 291.1268. $[\boldsymbol{\alpha}]_{D}^{27}=-72.5\left(\right.$ c $\left.0.26, \mathrm{CHCl}_{3}\right)$.

Determination of $\boldsymbol{e} e$ by Chiral SFC: Diacel CHIRALPAK IB N-3, $25{ }^{\circ} \mathrm{C}, 0.3 \mathrm{~cm} \phi, 15 \mathrm{~cm}$ column, $10 \% \mathrm{MeOH}$ in $\mathrm{CO}_{2}$, flow rate: $0.8 \mathrm{~mL} / \mathrm{min}$; $t \mathrm{R}: 5.05$ min (minor enantiomer), $5.66 \mathrm{~min}$ (major enantiomer); ee (major enantiomer $)=99 \%$.
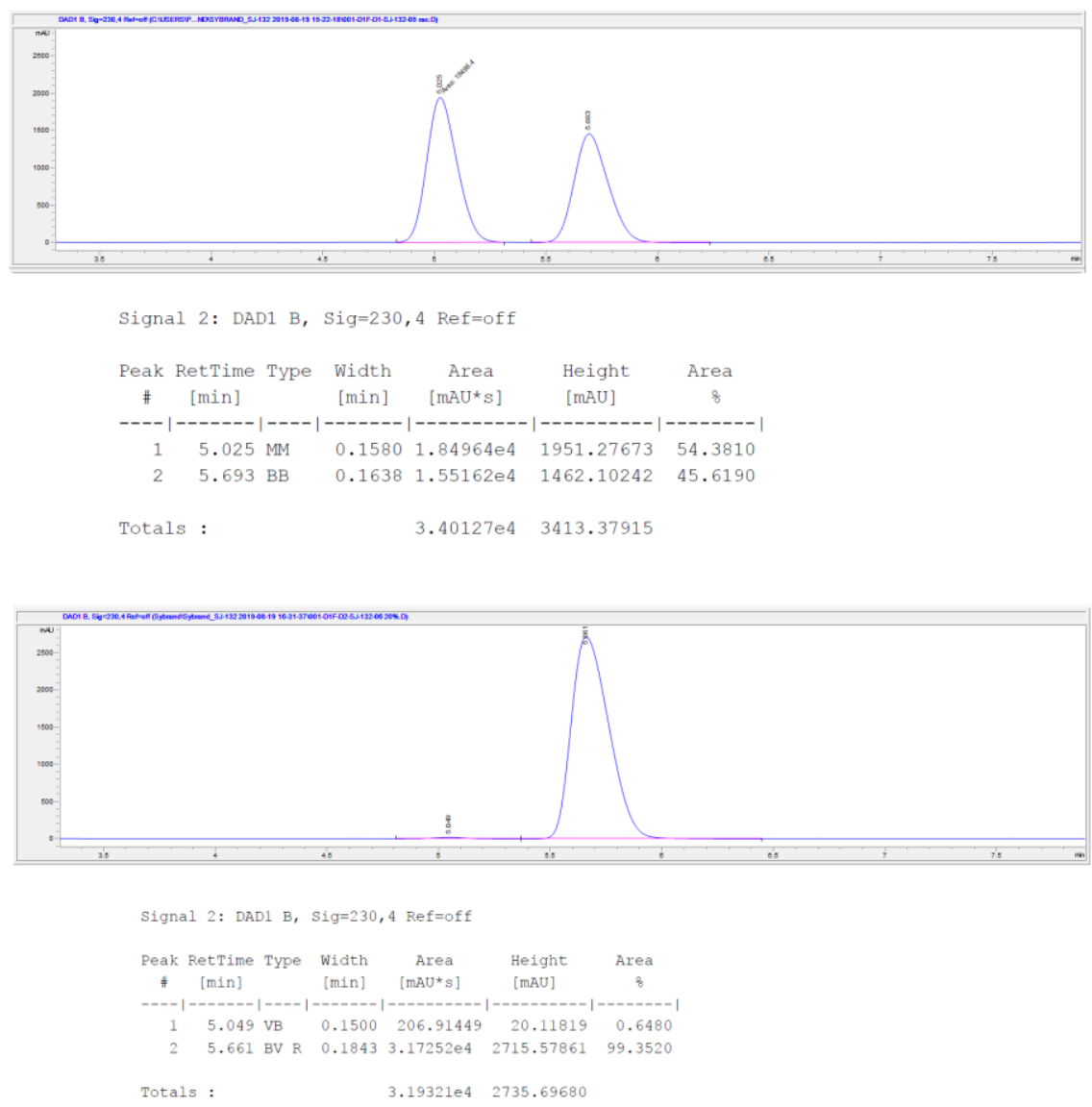
(E)-2-(1,1,1-trifluoro-5-phenylpent-3-en-2-yl)-2,3-dihydro-1H-naphtho[1,8-de][1,3,2]

diazaborinine (5c): This compound was obtained according to the above general procedure A.

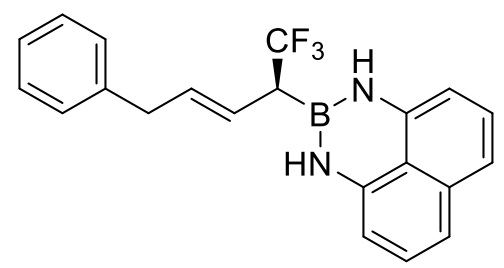
Product $\mathbf{5 c}$ was isolated in $59 \%$ yield $(21.6 \mathrm{mg}, 59 \mu \mathrm{mol})$ as light green oil by silica gel chromatography using pentane/ethyl acetate 20:1 as eluent.

${ }^{1}$ H NMR (400 MHz, $\left.\mathbf{C D C l}_{3}\right) \boldsymbol{\delta} 7.46-7.33(\mathrm{~m}, 3 \mathrm{H}), 7.33-7.22$ $(\mathrm{m}, 3 \mathrm{H}), 7.14(\mathrm{dd}, J=8.3,7.2 \mathrm{~Hz}, 2 \mathrm{H}), 7.08(\mathrm{dd}, J=8.4,1.2 \mathrm{~Hz}$, $2 \mathrm{H}), 6.32(\mathrm{dd}, J=7.2,1.2 \mathrm{~Hz}, 2 \mathrm{H}), 5.91(\mathrm{dt}, J=14.5,7.0 \mathrm{~Hz}, 1 \mathrm{H})$, $5.76(\mathrm{~s}, 2 \mathrm{H}), 5.62(\mathrm{ddt}, J=15.3,9.3,1.4 \mathrm{~Hz}, 1 \mathrm{H}), 3.49(\mathrm{~d}, J=6.9 \mathrm{~Hz}, 2 \mathrm{H}), 2.76(\mathrm{qd}, J=11.7,9.2$ $\mathrm{Hz}, 1 \mathrm{H}) ;{ }^{13} \mathbf{C}$ NMR $\left(\mathbf{1 0 1} \mathbf{M H z}, \mathbf{C D C l}_{\mathbf{3}}\right) \boldsymbol{\delta}$ 140.19, 139.92, 136.30, 135.86, 128.87, 128.57, 127.83 $\left(\mathrm{q},{ }^{1} J_{C-F}=277.7 \mathrm{~Hz}\right), 127.67,126.55,122.32\left(\mathrm{q},{ }^{3} J_{C-F}=3.8 \mathrm{~Hz}\right), 119.90,118.50,106.46,39.26$; ${ }^{19} \mathbf{F}$ NMR $\left(377 \mathbf{M H z}, \mathbf{C D C l}_{3}\right) \boldsymbol{\delta}-62.21(\mathrm{~d}, J=11.8 \mathrm{~Hz}) .{ }^{11} \mathbf{B}$ NMR $\left(\mathbf{1 2 8} \mathbf{~ M H z}, \mathbf{C D C l}_{3}\right) \boldsymbol{\delta}=28.64$. HRMS (neg. ESI) m/z: calcd for $\mathrm{C}_{21} \mathrm{H}_{17} \mathrm{BF}_{3} \mathrm{~N}_{2}[\mathrm{M}-\mathrm{H}]^{-} 365.1446$. Found 365.1447. $[\boldsymbol{\alpha}]_{\boldsymbol{D}}^{23}=-77.31$ (c $0.36, \mathrm{CHCl}_{3}$ ).

Determination of $\boldsymbol{e} e$ by Chiral SFC: Diacel CHIRALPAK IB N-3, $25{ }^{\circ} \mathrm{C}, 0.3 \mathrm{~cm} \phi, 15 \mathrm{~cm}$ column, $10 \% \mathrm{MeOH}$ in $\mathrm{CO}_{2}$, flow rate: $0.8 \mathrm{~mL} / \mathrm{min}$; $t \mathrm{R}: 16.51 \mathrm{~min}$ (minor enantiomer), $18.68 \mathrm{~min}$ (major enantiomer); ee (major enantiomer) $=96 \%$.

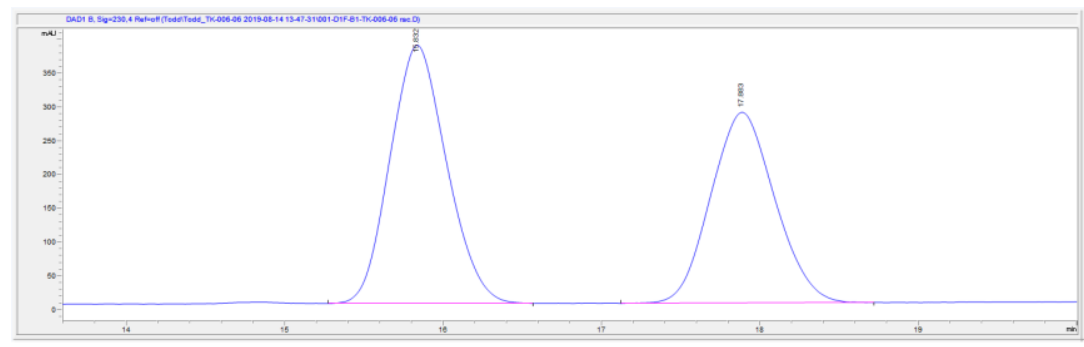

Signal 2: DAD1 B, Sig=230,4 Ref=off
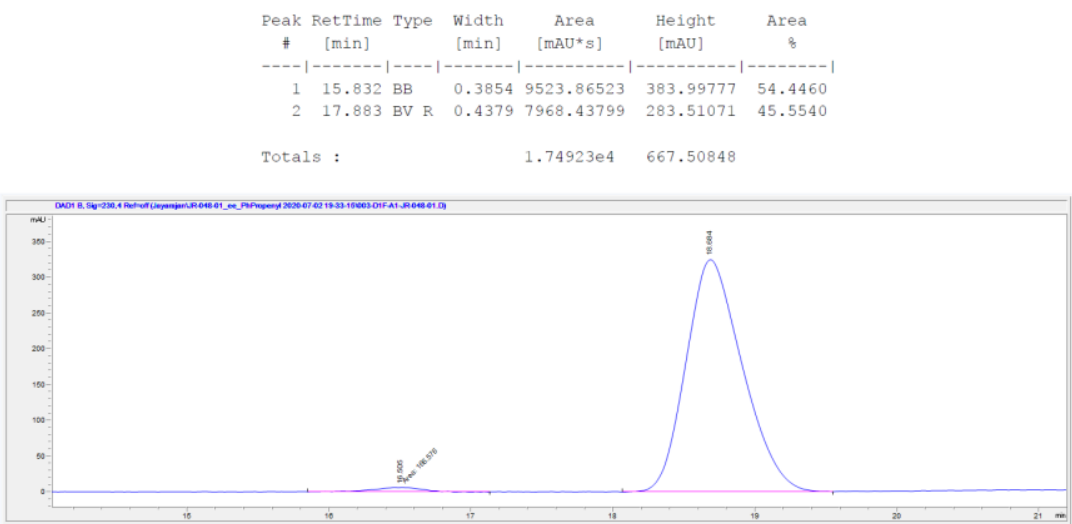

Signal 2: DAD1 B, Sig=230,4 Ref $=$ off

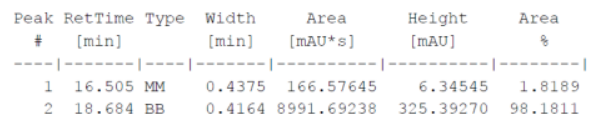

Totals : $\quad 9158.26883 \quad 331.73815$ 
diazaborinine (5d): This compound was obtained according to the above general procedure $\mathrm{A}$<smiles>FC(F)(/C=C/c1ccccc1)B1Nc2cccc3cccc(c23)N1</smiles>
using $30 \mathrm{~mol} \%$ of iodo-BINOL $4(0.03 \mathrm{mmol})$. Product 5d was isolated in $61 \%$ yield $(21.4 \mathrm{mg}, 61 \mu \mathrm{mol})$ as colourless viscous oil by silica gel chromatography using petroleum ether/ethyl acetate 40:1 as eluent. Use of $20 \mathrm{~mol} \%$ of iodo-BINOL $4(0.02 \mathrm{mmol})$ provided the product $\mathbf{5 d}$ in $54 \%$ yield $(19.1 \mathrm{mg}, 54 \mu \mathrm{mol})$ and $91 \%$ ee.

${ }^{1} \mathbf{H}$ NMR (400 MHz, CDCl$) \boldsymbol{\delta} 7.47-7.39(\mathrm{~m}, 2 \mathrm{H}), 7.41-7.32(\mathrm{~m}$, $2 \mathrm{H}), 7.34-7.26(\mathrm{~m}, 1 \mathrm{H}), 7.16-6.99(\mathrm{~m}, 4 \mathrm{H}), 6.63(\mathrm{~d}, J=15.8 \mathrm{~Hz}, 0 \mathrm{H}), 6.36(\mathrm{dd}, J=7.1,1.2 \mathrm{~Hz}$, 2H), $6.23(\mathrm{dd}, J=15.9,9.4 \mathrm{~Hz}, 1 \mathrm{H}), 5.81(\mathrm{~s}, 2 \mathrm{H}), 2.91(\mathrm{p}, J=11.5 \mathrm{~Hz}, 1 \mathrm{H}) ;{ }^{13} \mathbf{C} \mathbf{N M R}(\mathbf{1 0 1} \mathbf{~ M H z}$, $\left.\mathbf{C D C l}_{3}\right) \boldsymbol{\delta} 140.11,136.30,136.28,135.70,128.88,128.30,127.68,126.55,120.42\left(\mathrm{q},{ }^{3} J_{C-F}=4.1\right.$ $\mathrm{Hz}), 119.95,118.61,106.60 ;{ }^{19} \mathbf{F}$ NMR $\left(377 \mathbf{M H z}, \mathbf{C D C l}_{3}\right) \boldsymbol{\delta}-61.93(\mathrm{~d}, J=11.7 \mathrm{~Hz}) ;{ }^{11} \mathbf{B}$ NMR $\left(128 \mathrm{MHz}, \mathbf{C D C l}_{3}\right) \delta$ 29.58. HRMS (pos. APCI-ESI) m/z: calcd for $\mathrm{C}_{20} \mathrm{H}_{16} \mathrm{BF}_{3} \mathrm{~N}_{2}[\mathrm{M}+\mathrm{H}]^{+}$ 353.1435. Found 353.1422. $[\boldsymbol{\alpha}]_{D}^{27}=+54.4\left(c 0.25, \mathrm{CHCl}_{3}\right)$.

Determination of $e \boldsymbol{e}$ by Chiral SFC: Diacel CHIRALCEL OJ-H, $25^{\circ} \mathrm{C}, 0.3 \mathrm{~cm} \phi, 15 \mathrm{~cm}$ column, $20 \% \mathrm{MeOH}$ in $\mathrm{CO}_{2}$, flow rate: $1.2 \mathrm{~mL} / \mathrm{min}$; $t \mathrm{R}: 9.66$ min (major enantiomer), $11.01 \mathrm{~min}$ (minor enantiomer); ee (major enantiomer) $=94 \%$.

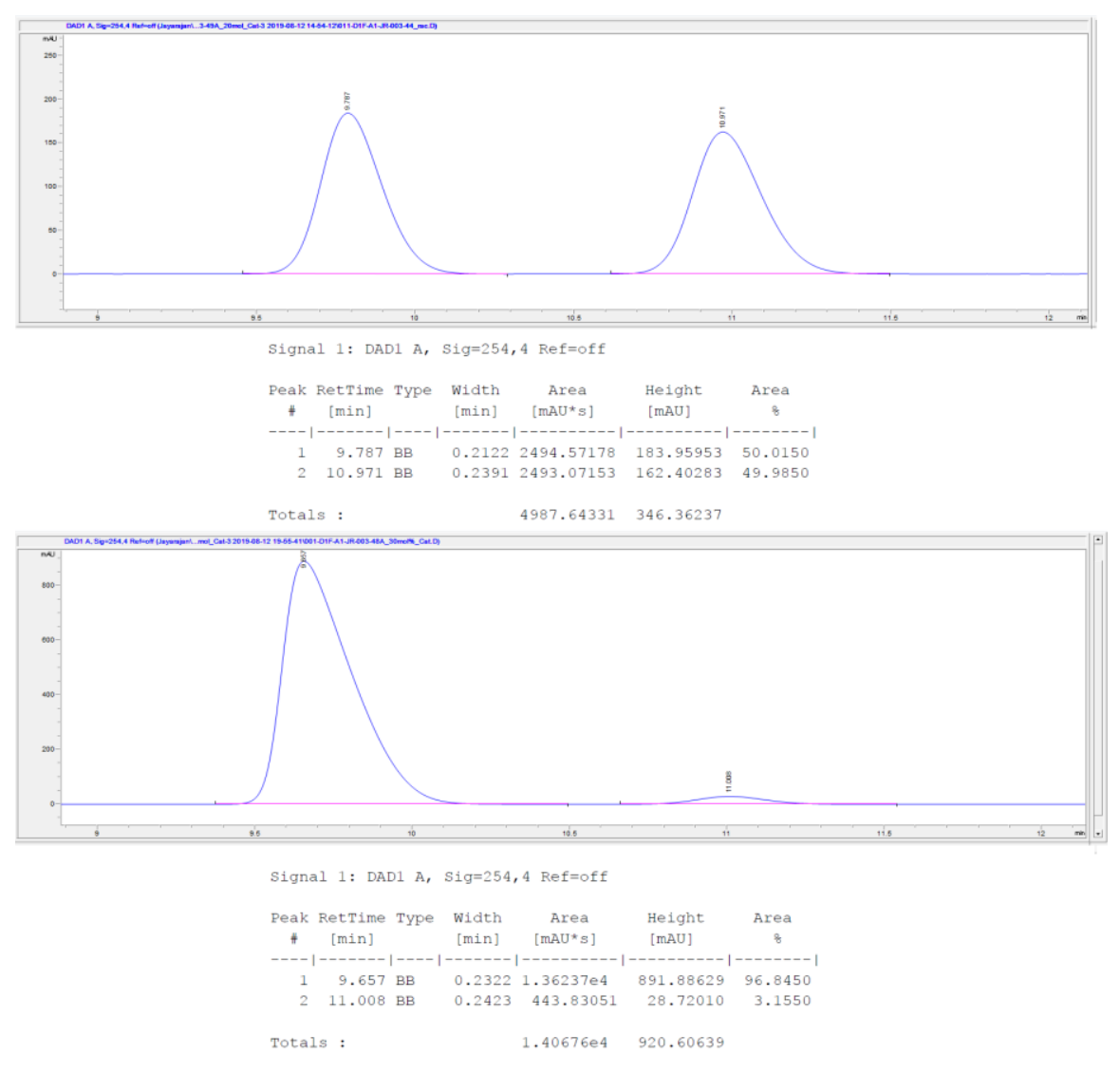


SFC result of product $5 \mathrm{~d}$ using $20 \mathrm{~mol} \%$ catalyst:

Determination of $\boldsymbol{e} e$ by Chiral SFC: Diacel CHIRALCEL OJ-H, $25^{\circ} \mathrm{C}, 0.3 \mathrm{~cm} \phi, 15 \mathrm{~cm}$ column, $20 \% \mathrm{MeOH}$ in $\mathrm{CO}_{2}$, flow rate: $1.2 \mathrm{~mL} / \mathrm{min}$; $t \mathrm{R}: 9.71 \mathrm{~min}$ (major enantiomer), $10.92 \mathrm{~min}$ (minor enantiomer); ee (major enantiomer $)=91 \%$.
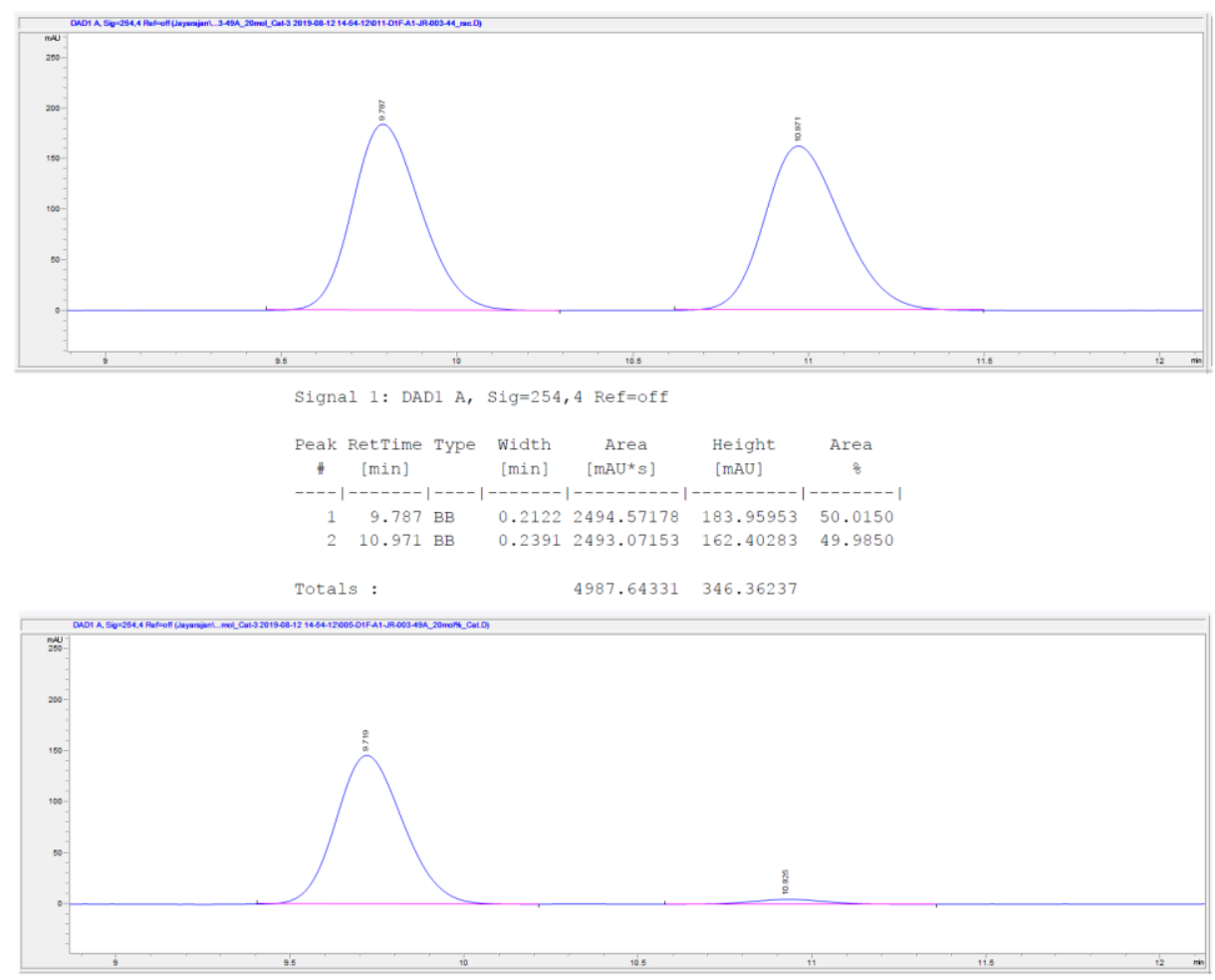

Signal 1: DAD1 A, Sig=254,4 Ref=off

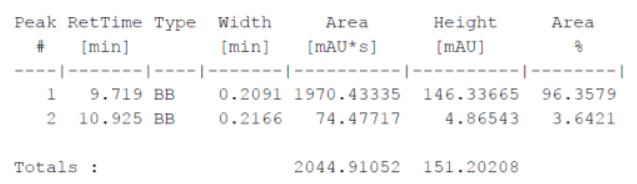


(S,E)-2-(4-(4-bromophenyl)-1,1,1-trifluorobut-3-en-2-yl)-2,3-dihydro-1H-naphtho[1,8-

de][1,3,2] diazaborinine (5e): This compound was obtained according to the above general<smiles>FC(F)(F)C(/C=C/c1ccc(Br)cc1)B1Nc2cccc3cccc(c23)N1</smiles>
procedure A using $30 \mathrm{~mol} \%$ of iodo-BINOL $4(0.03 \mathrm{mmol})$. Product 5e was isolated in $50 \%$ yield $(21.6 \mathrm{mg}, 50 \mu \mathrm{mol})$ as white solid by silica gel chromatography using petroleum ether/ethyl acetate $40: 1$ as eluent. M.pt: $142.5^{\circ} \mathrm{C}$.

Use of $20 \mathrm{~mol} \%$ of iodo-BINOL $4(0.02 \mathrm{mmol})$ provided the product $5 \mathbf{e}$ in $26 \%$ yield $(11.3 \mathrm{mg}, 26 \mu \mathrm{mol})$ and $89 \%$ ee.

${ }^{1}$ H NMR (400 MHz, CDCl $)$ ) $7.52-7.44(\mathrm{~m}, 2 \mathrm{H}), 7.31-7.27(\mathrm{~m}, 2 \mathrm{H}), 7.16-7.04(\mathrm{~m}, 4 \mathrm{H})$, $6.57(\mathrm{~d}, J=15.9 \mathrm{~Hz}, 1 \mathrm{H}), 6.35$ (dd, $J=7.1,1.3 \mathrm{~Hz}, 2 \mathrm{H}), 6.22(\mathrm{dd}, J=15.9,9.3 \mathrm{~Hz}, 1 \mathrm{H}), 5.79$ (s, 2H), $2.90(\mathrm{p}, J=11.1 \mathrm{~Hz}, 1 \mathrm{H}){ }^{\mathbf{1 3}} \mathbf{C}$ NMR (126 MHz, $\left.\mathbf{C D C l}_{\mathbf{3}}\right) \boldsymbol{\delta} 140.03,136.31,135.19,134.50$, 132.00, 128.74, 128.06, 127.70, 126.53, 122.16, $121.33\left(\mathrm{q},{ }^{3} J_{C-F}=3.7 \mathrm{~Hz}\right), 119.96,118.72,106.65$; ${ }^{19} \mathbf{F}$ NMR (377 MHz, $\left.\mathbf{C D C l}_{3}\right) \boldsymbol{\delta}-61.96(\mathrm{~d}, J=11.3 \mathrm{~Hz}) ;{ }^{11} \mathbf{B} \mathbf{N M R}\left(\mathbf{1 6 0} \mathbf{M H z}, \mathbf{C D C l}_{3}\right) \boldsymbol{\delta} 28.30$. HRMS (pos. APCI-ESI) m/z: calcd for $\mathrm{C}_{20} \mathrm{H}_{16} \mathrm{BBrF}_{3} \mathrm{~N}_{2}[\mathrm{M}+\mathrm{H}]^{+}$431.0540. Found 431.0534. $[\boldsymbol{\alpha}]_{D}^{27}=+64.4\left(c 0.25, \mathrm{CHCl}_{3}\right)$.

Determination of $e e$ by Chiral SFC: Diacel CHIRALCEL OJ-H, $25^{\circ} \mathrm{C}, 0.3 \mathrm{~cm} \phi, 15 \mathrm{~cm}$ column, $20 \% \mathrm{MeOH}$ in $\mathrm{CO}_{2}$, flow rate: $1.2 \mathrm{~mL} / \mathrm{min}$; $t \mathrm{R}: 16.26$ min (major enantiomer), 21.83 min (minor enantiomer); ee (major enantiomer) $=94 \%$. (Sample was prepared in $\left.\mathrm{CDCl}_{3}\right)$
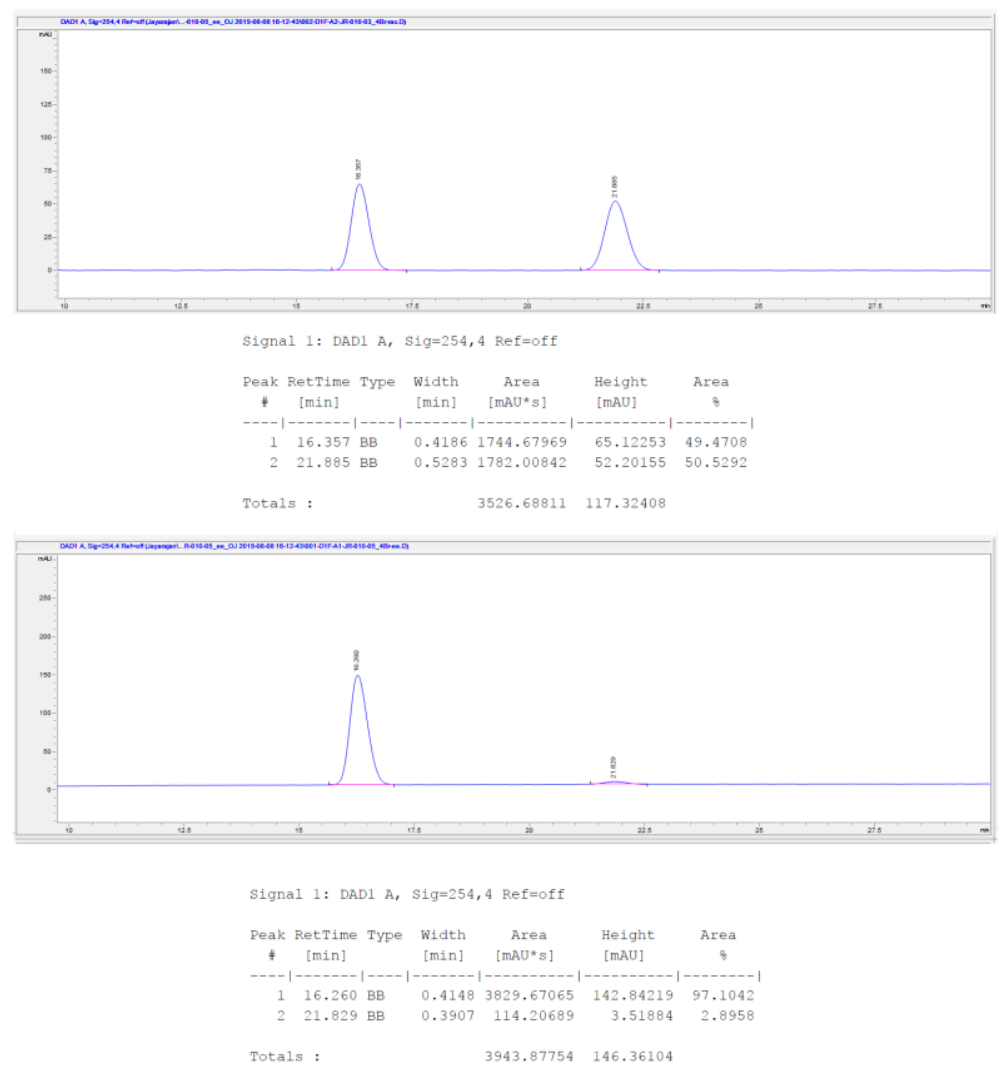


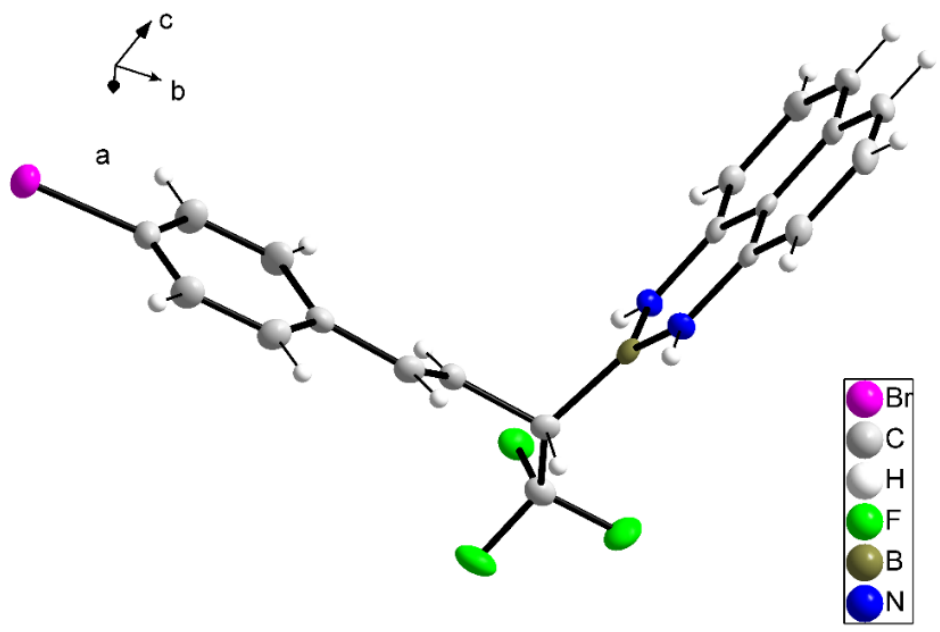

Figure S2 X-ray structure of 5e (CCDC number: 1951527). See the attached Xray5e.cif file.

SFC result of product $5 \mathrm{e}$ using $20 \mathrm{~mol} \%$ catalyst:

Determination of $e$ e by Chiral SFC: Diacel CHIRALCEL OJ-H, $25^{\circ} \mathrm{C}, 0.3 \mathrm{~cm} \phi, 15 \mathrm{~cm}$ column, $20 \% \mathrm{MeOH}$ in $\mathrm{CO}_{2}$, flow rate: $1.2 \mathrm{~mL} / \mathrm{min}$; $t \mathrm{R}: 15.68 \mathrm{~min}$ (major enantiomer), 21.62 min (minor enantiomer); ee (major enantiomer) $=89 \%$. (Sample was prepared in $\mathrm{CDCl}_{3}$ )
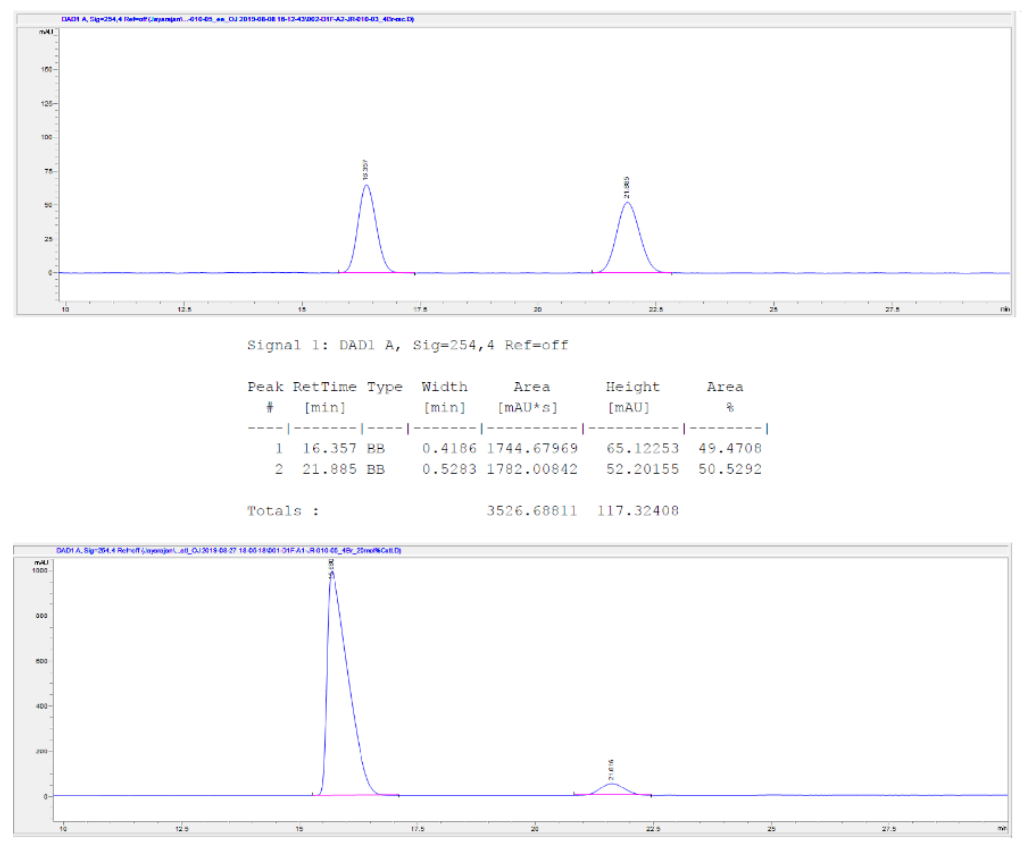

Signal 1: DAD1 A, Sig=254,4 Ref=off

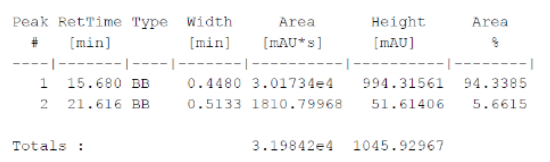




\subsection{Stereoinduction model for the homologation reaction}

A plausible stereoinduction model is given in Figure S3. According to this the $\mathrm{C}_{2}$-symmetrical BINOL vinyl-boronate ester $\mathbf{A}$ (see Figure 2 in the paper) reacts with $\mathrm{CF}_{3}$-diazomethane $\mathbf{3}$. The Si-face attack by $\mathbf{3}$ leads formation to ate complex (S)-B, while the Re-face attack affords (R)-B. In $(\mathrm{R})-\mathrm{B}$ the $\mathrm{CF}_{3}$ group and the iodine of BINOL have an unfavorable steric hindrance. Therefore, the reaction most probably proceeds via (S)-B, which undergoes antiperiplanar 1,2-migration of the vinyl group to give (S)-C. Subsequent hydrolysis leads to 1e with (S) configuration, which was obtained as the major enantiomer in the homologation reaction.

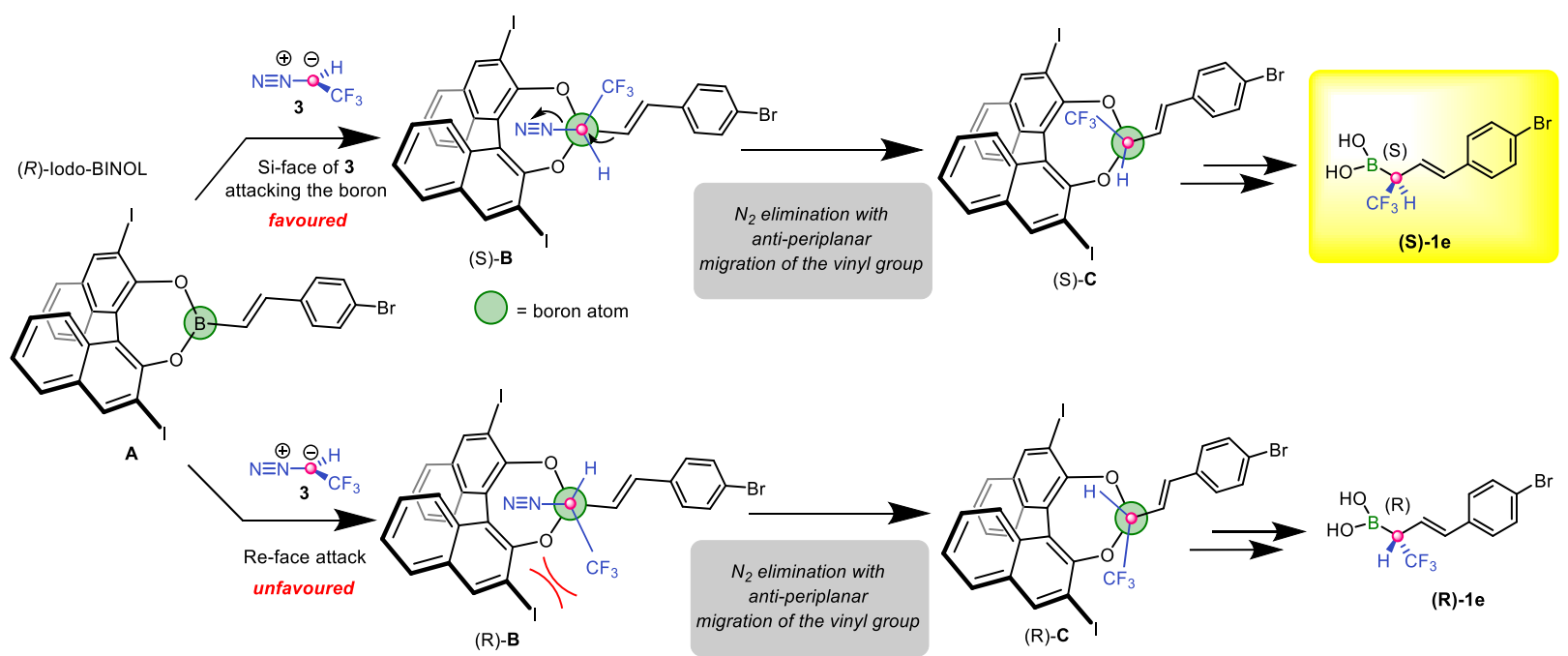

Figure S3. Stereoinduction model for the asymmetric homologation. 
(S,E)-2-(4-(4-chlorophenyl)-1,1,1-trifluorobut-3-en-2-yl)-2,3-dihydro-1H-naphtho[1,8de][1,3,2]diazaborinine (5f): This compound was obtained according to the above general<smiles>FC(F)(F)C(/C=C/c1ccc(Cl)cc1)C(F)(F)C(F)(F)F</smiles>
procedure A using $30 \mathrm{~mol} \%$ of iodo-BINOL $4(0.03 \mathrm{mmol})$. Product $\mathbf{5 f}$ was isolated in $70 \%$ yield $(26.9 \mathrm{mg}, 70 \mu \mathrm{mol})$ as light green oil by silica gel chromatography using pentane/DCM 5:1 as eluent.

${ }^{1} \mathbf{H}$ NMR (400 MHz, $\left.\mathbf{C D C l}_{3}\right) \boldsymbol{\delta} 7.37-7.28(\mathrm{~m}, 4 \mathrm{H}), 7.16-7.05$ $(\mathrm{m}, 4 \mathrm{H}), 6.58(\mathrm{~d}, J=15.9 \mathrm{~Hz}, 1 \mathrm{H}), 6.36(\mathrm{dd}, J=7.1,1.3 \mathrm{~Hz}, 2 \mathrm{H})$, $6.20(\mathrm{dd}, J=15.9,9.4 \mathrm{~Hz}, 1 \mathrm{H}), 5.79(\mathrm{~s}, 2 \mathrm{H}), 2.89(\mathrm{p}, J=11.3 \mathrm{~Hz}, 1 \mathrm{H}) ;{ }^{13} \mathbf{C}$ NMR $(\mathbf{1 0 1} \mathbf{M H z}$, $\left.\mathbf{C D C l}_{3}\right) \boldsymbol{\delta}=140.04,136.31,134.75,134.43,134.00,129.63,129.04,128.03,127.75,127.70$, $126.29,124.74,121.16\left(\mathrm{q},{ }^{3} J_{C-F}=3.7 \mathrm{~Hz}\right), 119.95,118.70,106.64,38.96 ;{ }^{19} \mathbf{F}$ NMR $(377 \mathbf{~ M H z}$, $\left.\mathbf{C D C l}_{3}\right) \boldsymbol{\delta}-61.95(\mathrm{~d}, J=11.5 \mathrm{~Hz}) ;{ }^{11} \mathbf{B}$ NMR $\left(\mathbf{1 2 8} \mathbf{M H z}, \mathbf{C D C l}_{\mathbf{3}}\right) \boldsymbol{\delta}=129.81$. After repeated attempts we were unable to obtain HMRS data for compound $\mathbf{5 f}$, which is probably due to its unstable nature and its resistance to ionization. $[\alpha]_{D}^{27}=+54.0\left(c 0.25, \mathrm{CHCl}_{3}\right)$.

Determination of $\boldsymbol{e} \boldsymbol{e}$ by Chiral SFC: Daicel CHIRALCEL OJ-H, $25^{\circ} \mathrm{C}, 0.46 \mathrm{~cm} \phi, 10 \mathrm{~cm}$ column, $20 \% \mathrm{MeOH}$ in $\mathrm{CO}_{2}$, flow rate: $1.2 \mathrm{~mL} / \mathrm{min}$; $t \mathrm{R}: 11.88 \mathrm{~min}$ (major enantiomer), $15.29 \mathrm{~min}$ (minor enantiomer); ee (major enantiomer) $=86 \%$.

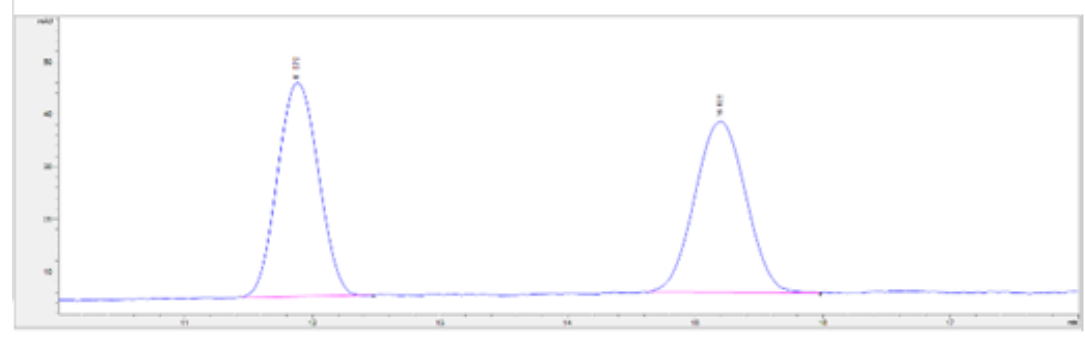

Signal 1: DAD1 A, Sigm 254,4 Ref=off
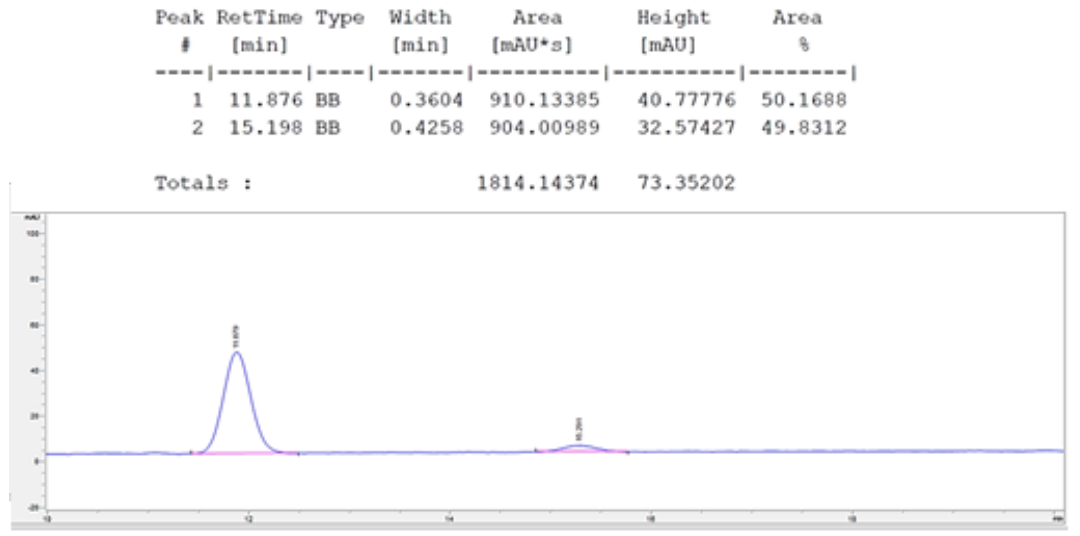

Signal 1: DAD1 A, Sig-254,4 Ref-off

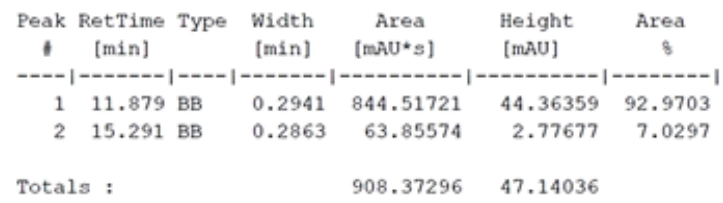


(S,E)-2-(1,1,1-trifluoro-4-(4-(trifluoromethyl)phenyl)but-3-en-2-yl)-2,3-dihydro-1H-

naphtho[1,8-de][1,3,2]diazaborinine (5g): This compound was obtained according to the above<smiles>FC(F)(F)c1ccc(/C=C/C(B2Nc3cccc4cccc(c34)N2)C(F)(F)F)cc1</smiles>
general procedure A using $30 \mathrm{~mol} \%$ of iodo-BINOL 4 (0.03 mmol) at $30{ }^{\circ} \mathrm{C}$ for $48 \mathrm{~h}$. Product $5 \mathrm{~g}$ was isolated in $51 \%$ yield $(21.4 \mathrm{mg}, 51 \mu \mathrm{mol})$ as colourless viscous oil by silica gel chromatography using petroleum ether/ethyl acetate $40: 1$ as eluent.

${ }^{1} \mathbf{H}$ NMR $\left(400 \mathrm{MHz}, \mathbf{C D C l}_{3}\right) \boldsymbol{\delta} 7.61(\mathrm{~d}, J=8.2 \mathrm{~Hz}, 2 \mathrm{H}), 7.51$ $(\mathrm{d}, J=8.5 \mathrm{~Hz}, 2 \mathrm{H}), 7.15-7.01(\mathrm{~m}, 4 \mathrm{H}), 6.67(\mathrm{~d}, J=15.9 \mathrm{~Hz}, 1 \mathrm{H}), 6.41-6.27(\mathrm{~m}, 3 \mathrm{H}), 5.79(\mathrm{~s}$, 2H), $2.95(\mathrm{p}, J=11.2 \mathrm{~Hz}, 1 \mathrm{H}) ;{ }^{\mathbf{1 3}} \mathbf{C}$ NMR (101 MHz, $\left.\mathbf{C D C l}_{3}\right) \boldsymbol{\delta}$ 139.97, 139.65, 136.31, 134.27, $130.13\left(\mathrm{q},{ }^{2} J_{C-F}=32.5 \mathrm{~Hz}\right), 128.96,127.71,126.74,126.20,125.85\left(\mathrm{q},{ }^{3} J_{C-F}=3.8 \mathrm{~Hz}\right), 125.56(\mathrm{q}$, $\left.{ }^{1} J_{C-F}=272.0 \mathrm{~Hz}\right), 123.37\left(\mathrm{q},{ }^{3} J_{C-F}=3.8 \mathrm{~Hz}\right), 119.97,118.79,106.70 ;{ }^{19} \mathbf{F} \mathbf{N M R}\left(377 \mathbf{~ M H z}, \mathbf{C D C l}_{\mathbf{3}}\right)$ $\boldsymbol{\delta}-61.90(\mathrm{~d}, J=11.4 \mathrm{~Hz}),-62.60 .{ }^{11} \mathbf{B}$ NMR $\left(128 \mathrm{MHz}, \mathbf{C D C l}_{3}\right) \boldsymbol{\delta} 28.83$. HRMS (pos. APCIESI) $\mathbf{m} / \mathbf{z}$ : calcd for $\mathrm{C}_{21} \mathrm{H}_{16} \mathrm{BF}_{6} \mathrm{~N}_{2}[\mathrm{M}+\mathrm{H}]^{+}$421.1309. Found 421.1311. $[\boldsymbol{\alpha}]_{\boldsymbol{D}}^{27}=+41.20(c$ 0.25, $\left.\mathrm{CHCl}_{3}\right)$.

Determination of $e$ by Chiral SFC: Diacel CHIRALCEL OJ-H, $25^{\circ} \mathrm{C}, 0.3 \mathrm{~cm} \phi, 15 \mathrm{~cm}$ column, $20 \% \mathrm{MeOH}$ in $\mathrm{CO}_{2}$, flow rate: $1.2 \mathrm{~mL} / \mathrm{min} ; \mathrm{R}: 4.31 \mathrm{~min}$ (major enantiomer), 6.34 min (minor enantiomer); ee (major enantiomer) $=90 \%$.
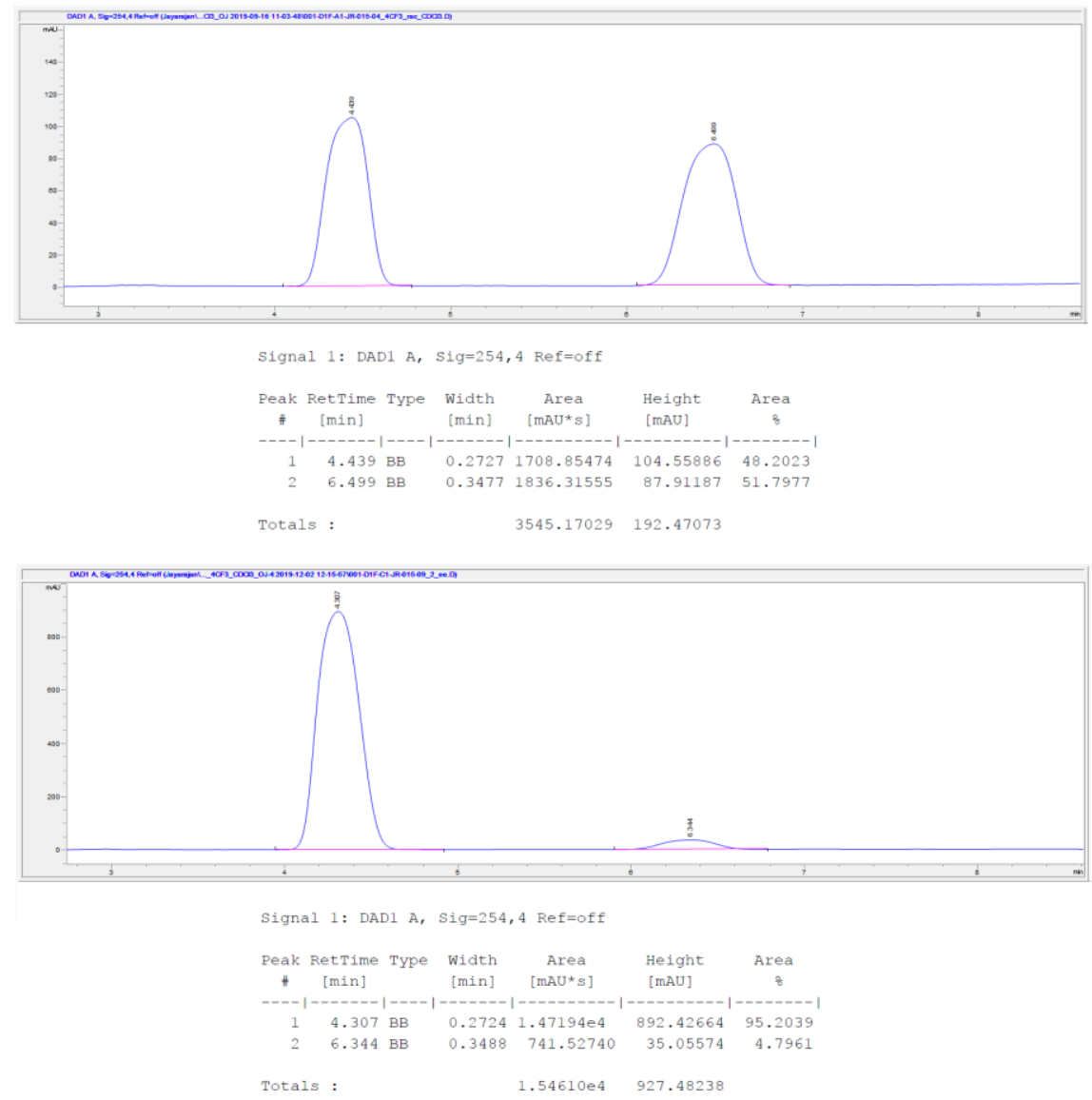
(1S,2S)-1-(4-bromophenyl)-2-(3,3,3-trifluoropropyl)octan-1-ol (7a): This compound was<smiles>CCCCCCC(/C=C/C(F)(F)F)C(O)c1ccc(Br)cc1</smiles>
obtained according to the above general procedure C. Product 1a was isolated in $60 \%$ yield $(22.7 \mathrm{mg}, 60 \mu \mathrm{mol})$ as a colorless oil by silica gel chromatography using pentane/ethyl acetate 100:1 as eluent.

${ }^{1} \mathbf{H}$ NMR (400 MHz, $\left.\mathbf{C D C l}_{3}\right) \boldsymbol{\delta} 7.51-7.44(\mathrm{~m}, 2 \mathrm{H}), 7.20$ - $7.12(\mathrm{~m}$, 2H), $6.25(\mathrm{ddq}, J=15.9,9.5,2.1 \mathrm{~Hz}, 1 \mathrm{H}), 5.57(\mathrm{dqd}, J=15.9,6.3,0.8 \mathrm{~Hz}, 1 \mathrm{H}), 4.60(\mathrm{~d}, J=6.1$ $\mathrm{Hz}, 1 \mathrm{H}), 2.34$ (dddd, $J=10.4,9.0,6.2,2.8 \mathrm{~Hz}, 1 \mathrm{H}), 1.83(\mathrm{~s}, 1 \mathrm{H}), 1.46-1.04(\mathrm{~m}, 10 \mathrm{H}), 0.86(\mathrm{t}, J$ $=6.9 \mathrm{~Hz}, 3 \mathrm{H}) ;{ }^{13} \mathbf{C}$ NMR $\left(\mathbf{1 0 1} \mathbf{~ M H z}, \mathbf{C D C l}_{3}\right) \boldsymbol{\delta} 141.41,140.42\left(\mathrm{q},{ }^{3} J_{C-F}=6.4 \mathrm{~Hz}\right), 131.67,128.31$, $122.79\left(\mathrm{q},{ }^{1} J_{C-F}=269.6 \mathrm{~Hz}\right), 121.89,121.37\left(\mathrm{q},{ }^{2} J_{C-F}=33.4 \mathrm{~Hz}\right), 76.10,50.05,31.76,30.64,29.23$, 27.16, 22.70, 14.17. ${ }^{19} \mathbf{F}$ NMR (377 MHz, $\left.\mathbf{C D C l}_{3}\right) \delta-63.89$ (dd, $J=6.2,2.0 \mathrm{~Hz}$ ); HRMS (neg. ESI) $\mathbf{m} / \mathbf{z}$ : calcd for $\mathrm{C}_{18} \mathrm{H}_{23} \mathrm{BrF}_{3} \mathrm{O}_{3}\left[\mathrm{M}-\mathrm{H}+\mathrm{CH}_{2} \mathrm{O}_{2}\right]^{-} 423.0788$. Found 423.0804. $[\boldsymbol{\alpha}]_{\boldsymbol{D}}^{24}=-32.3(c$ $\left.0.30, \mathrm{CHCl}_{3}\right)$.

Determination of $e \boldsymbol{e}$ by Chiral SFC: Diacel CHIRALPAK IA-3, $25^{\circ} \mathrm{C}, 0.3 \mathrm{~cm} \phi, 15 \mathrm{~cm}$ column, $0.5 \% \mathrm{MeOH}$ in $\mathrm{CO}_{2}$, flow rate: $1.8 \mathrm{~mL} / \mathrm{min}$; $t \mathrm{R}: 6.09$ min (minor enantiomer), 7.50 min (major enantiomer); $e e($ major enantiomer $)=98 \%$.

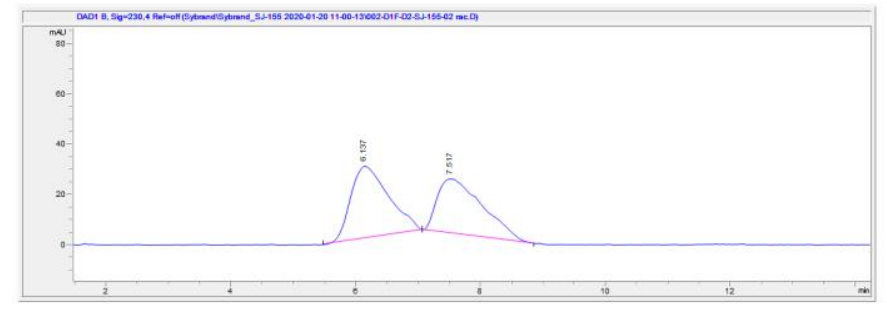

Signal 2: DAD1 B, Sig=230,4 Ref=off
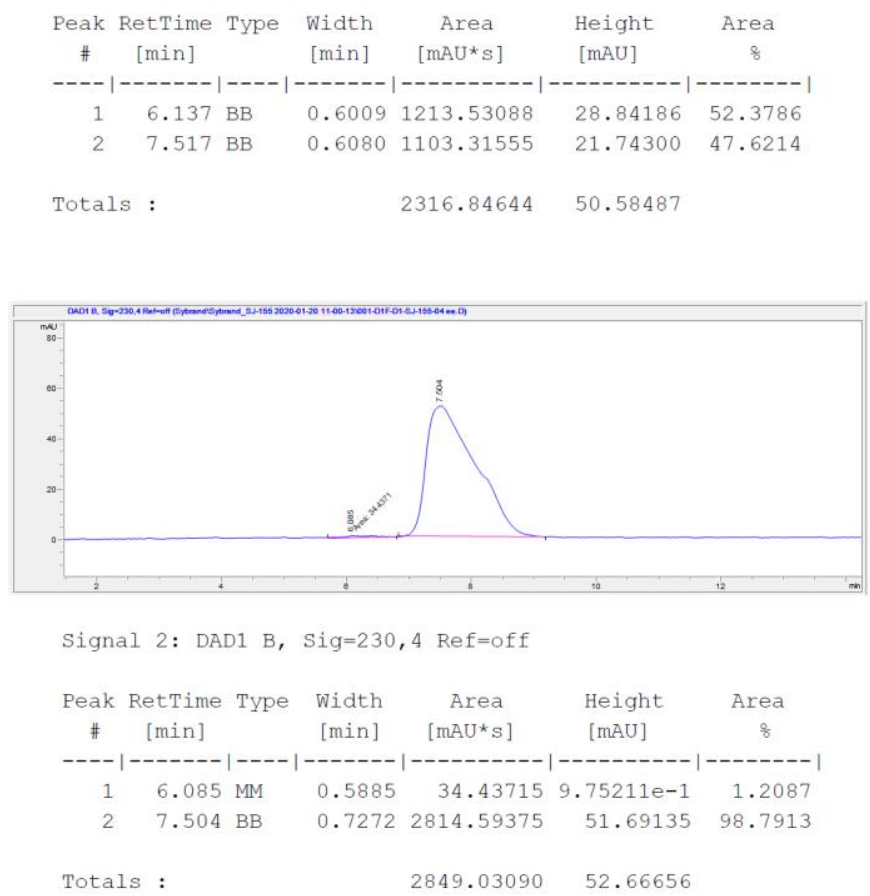
(1S,2S,E)-2-benzyl-1-(4-bromophenyl)-5,5,5-trifluoropent-3-en-1-ol (7b): This compound was<smiles>OC(/C=C/C(F)(F)F)C(Cc1ccccc1)c1ccc(Br)cc1</smiles>
obtained according to the above general procedure C. Product $\mathbf{7 b}$ was isolated in $47 \%$ yield $(18.1 \mathrm{mg}, 47 \mu \mathrm{mol})$ as colourless oil by silica gel chromatography using pentane/ethyl acetate $30: 1$ as eluent.

${ }^{1} \mathbf{H}$ NMR (400 MHz, $\left.\mathbf{C D C l}_{3}\right) \boldsymbol{\delta} 7.52-7.43(\mathrm{~m}, 2 \mathrm{H}), 7.34-7.24(\mathrm{~m}$, $2 \mathrm{H}), 7.26-7.17(\mathrm{~m}, 1 \mathrm{H}), 7.18-7.07(\mathrm{~m}, 4 \mathrm{H}), 6.40-6.27(\mathrm{~m}, 1 \mathrm{H})$, $5.32(\mathrm{dqd}, J=15.9,6.2,0.8 \mathrm{~Hz}, 1 \mathrm{H}), 4.68(\mathrm{~d}, J=4.3 \mathrm{~Hz}, 1 \mathrm{H}), 2.94-$ $2.80(\mathrm{~m}, 1 \mathrm{H}), 2.76-2.56(\mathrm{~m}, 2 \mathrm{H}), 1.91(\mathrm{~s}, 1 \mathrm{H}) ;{ }^{13} \mathbf{C} \mathbf{~ N M R}\left(\mathbf{1 0 1} \mathbf{~ M H z}, \mathbf{C D C l}_{\mathbf{3}}\right) \boldsymbol{\delta} \mathbf{1 4 1 . 4 4}, 138.83$ $\left(\mathrm{q},{ }^{3} J_{C-F}=6.5 \mathrm{~Hz}\right), 131.70,129.22,128.68,128.05,126.67,122.64\left(\mathrm{~d},{ }^{1} J_{C-F}=269.7 \mathrm{~Hz}\right), 121.88$, $121.59\left(\mathrm{q},{ }^{2} J_{C-F}=33.6 \mathrm{~Hz}\right), 74.54,51.54,37.44 ;{ }^{19} \mathbf{F} \mathbf{N M R}\left(\mathbf{3 7 7} \mathbf{~ M H z}, \mathbf{C D C l}_{\mathbf{3}}\right) \boldsymbol{\delta}-64.13(\mathrm{dd}, J=$ 6.1, $1.9 \mathrm{~Hz}$ ). HRMS (neg. ESI) m/z: calcd for $\mathrm{C}_{19} \mathrm{H}_{17} \mathrm{BrF}_{3} \mathrm{O}_{3}\left[\mathrm{M}-\mathrm{H}+\mathrm{CH}_{2} \mathrm{O}_{2}\right]^{-} 429.0319$. Found

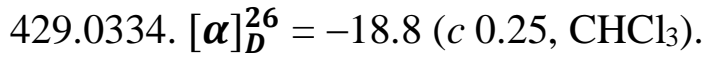

Determination of $e \boldsymbol{e}$ by Chiral SFC: Daicel CHIRALPAK IA-3, $25^{\circ} \mathrm{C}, 0.3 \mathrm{~cm} \phi, 15 \mathrm{~cm}$ column, $5 \% \mathrm{MeOH}$ in $\mathrm{CO}_{2}$, flow rate: $0.8 \mathrm{~mL} / \mathrm{min}$; $t \mathrm{R}: 3.90 \mathrm{~min}$ (minor enantiomer), 5.84 min (major enantiomer); ee (major enantiomer) $=94 \%$.
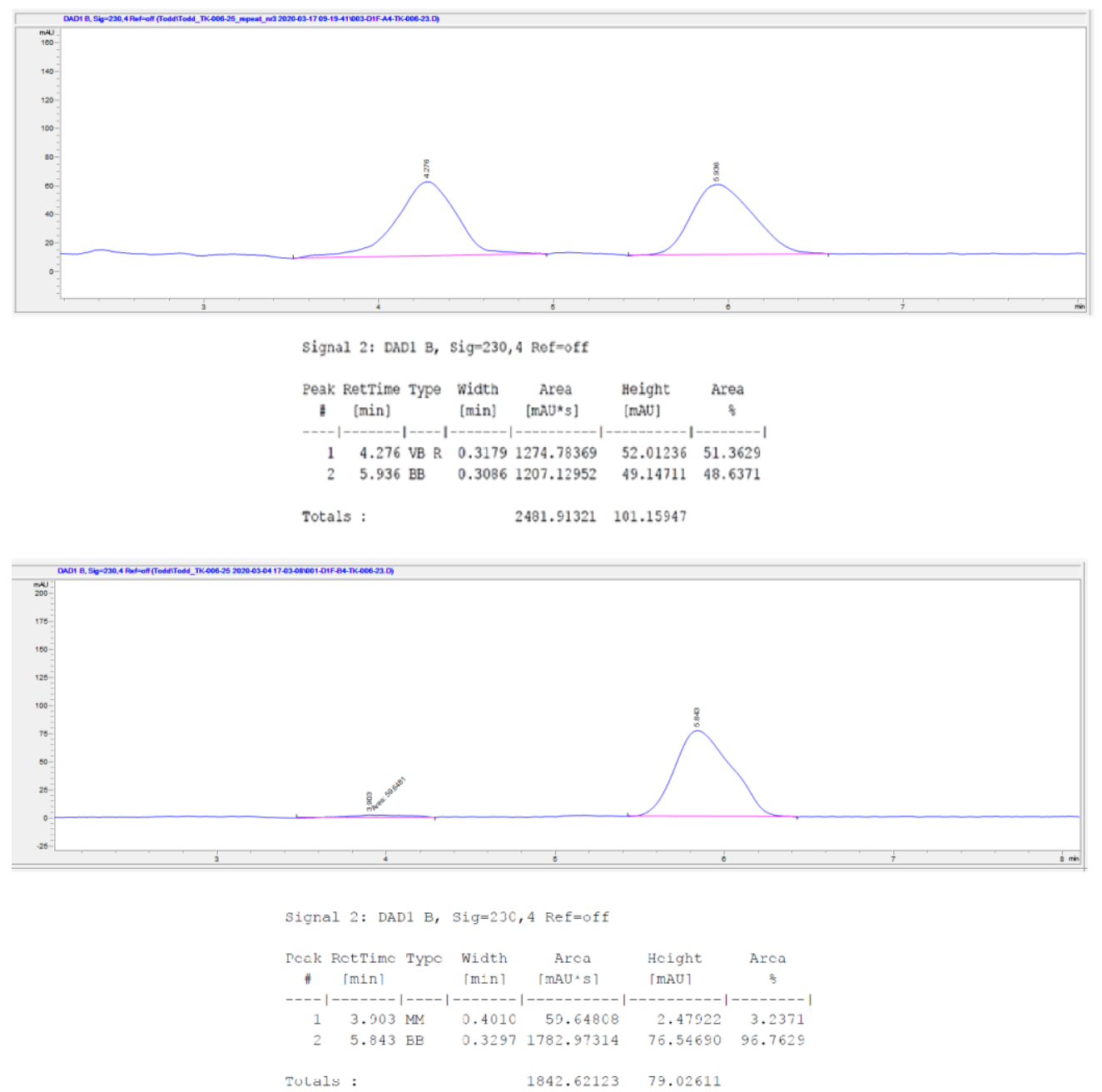
(1S,2R,E)-1-(4-bromophenyl)-5,5,5-trifluoro-2-phenylpent-3-en-1-ol (7c): This compound<smiles>OC(c1ccc(Br)cc1)C(/C=C/C(F)(F)F)c1ccccc1</smiles>
was obtained according to the above general procedure $\mathrm{C}$ using 30 mol\% of iodo-BINOL $4(0.03 \mathrm{mmoL})$. Product $7 \mathbf{c}$ was isolated in $41 \%$ yield $(15.1 \mathrm{mg}, 41 \mu \mathrm{mol})$ as colorless oil by silica gel chromatography using petroleum ether/ethyl acetate 40:1 as eluent. Product 7c was isolated in $53 \%$ yield $(19.5 \mathrm{mg}, 53 \mu \mathrm{mol})$ with general procedure $\mathrm{E}$ except that the reaction was done for 4 hours.

${ }^{1} \mathbf{H}$ NMR (400 MHz, CDCl $)$ ) $7.45-7.37(\mathrm{~m}, 2 \mathrm{H}), 7.34-7.23(\mathrm{~m}, 3 \mathrm{H}), 7.14-7.10(\mathrm{~m}, 2 \mathrm{H})$, $7.09-7.03(\mathrm{~m}, 2 \mathrm{H}), 6.87$ (ddq, $J=15.8,8.2,2.1 \mathrm{~Hz}, 1 \mathrm{H}), 5.65(\mathrm{dqd}, J=15.9,6.3,1.2 \mathrm{~Hz}, 1 \mathrm{H})$, $4.95(\mathrm{dd}, J=7.0,3.0 \mathrm{~Hz}, 1 \mathrm{H}), 3.65(\mathrm{ddt}, J=8.4,6.9,1.6 \mathrm{~Hz}, 1 \mathrm{H}), 2.15(\mathrm{~d}, J=3.1 \mathrm{~Hz}, 1 \mathrm{H}) ;{ }^{13} \mathbf{C}$ NMR (101 MHz, $\left.\mathbf{C D C l}_{3}\right) \boldsymbol{\delta} 140.80,139.14\left(\mathrm{q},{ }^{3} J_{C-F}=6.5 \mathrm{~Hz}\right), 138.69,131.47,128.97,128.57$, $128.21,127.61,127.03,122.93\left(\mathrm{q},{ }^{4} J_{C-F}=269.7 \mathrm{~Hz}\right), 121.86,121.38\left(\mathrm{q},{ }^{2} J_{C-F}=33.5 \mathrm{~Hz}\right), 76.84$, 56.07; ${ }^{19} \mathbf{F}$ NMR (377 MHz, $\left.\mathbf{C D C l}_{3}\right) \boldsymbol{\delta}-64.00$ (dt, $\left.J=6.2,1.7 \mathrm{~Hz}\right)$. HRMS (neg. ESI) m/z: calcd for $\mathrm{C}_{18} \mathrm{H}_{15} \mathrm{BrF}_{3} \mathrm{O}_{3}\left[\mathrm{M}+\mathrm{CH}_{2} \mathrm{O}_{2}-\mathrm{H}\right]^{-}$415.0162. Found 415.0160. $[\alpha]_{D}^{26}=+9.6\left(c 0.25, \mathrm{CHCl}_{3}\right)$.

Determination of $e \boldsymbol{e}$ by Chiral SFC: Diacel CHIRALPAK IA-3, $25^{\circ} \mathrm{C}, 0.3 \mathrm{~cm} \phi, 15 \mathrm{~cm}$ column, $1 \% \mathrm{MeOH}$ in $\mathrm{CO}_{2}$, flow rate: $1.2 \mathrm{~mL} / \mathrm{min}$; $t \mathrm{R}: 12.54 \mathrm{~min}$ (minor enantiomer), 14.77 min (major enantiomer); $e e$ (major enantiomer) $=90 \%$.
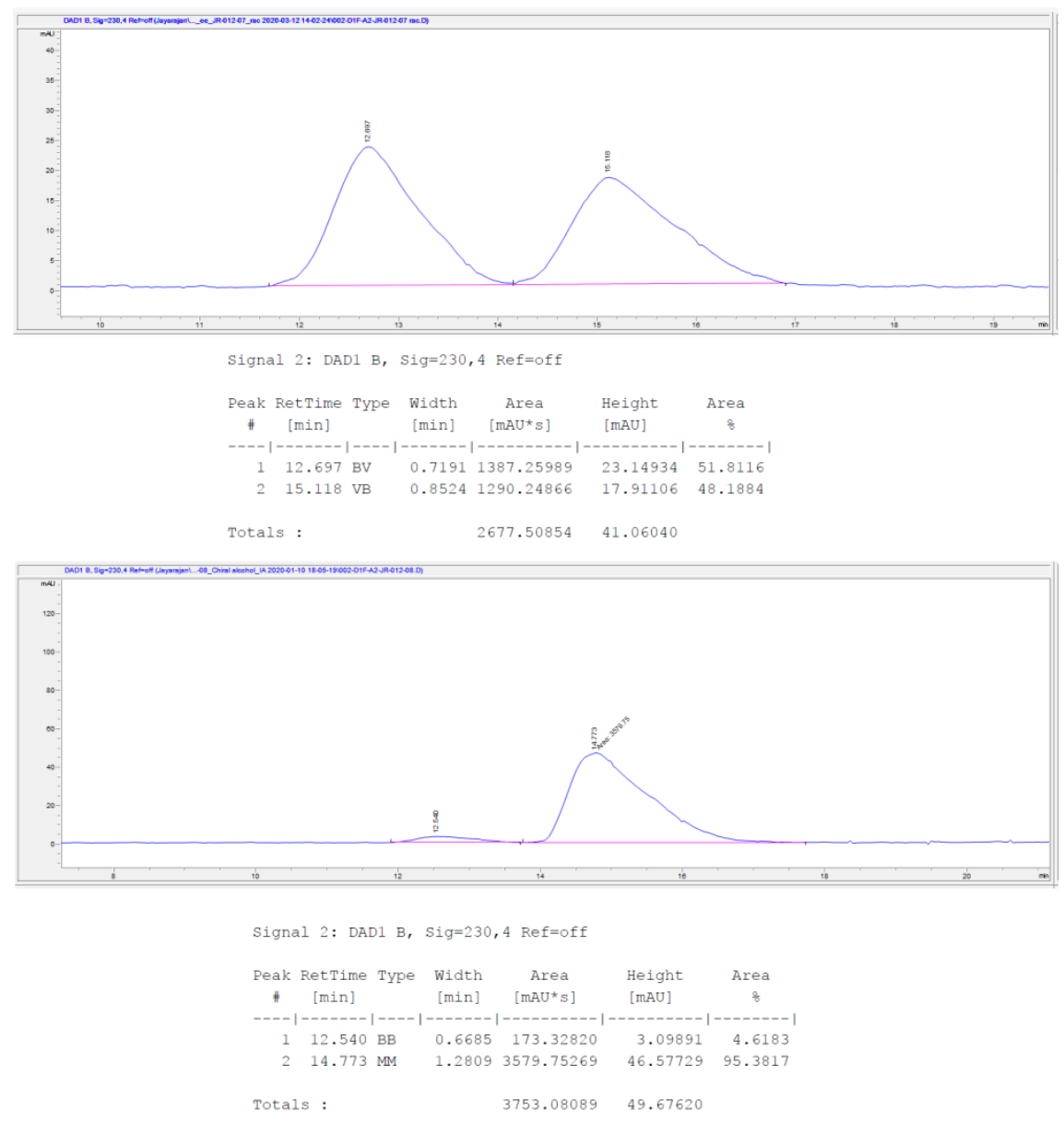
Determination of $\boldsymbol{e} e$ by Chiral SFC: Diacel CHIRALPAK IA N-3, $25{ }^{\circ} \mathrm{C}, 0.3 \mathrm{~cm} \phi, 15 \mathrm{~cm}$ column, $1 \% \mathrm{MeOH}$ in $\mathrm{CO}_{2}$, flow rate: $1.2 \mathrm{~mL} / \mathrm{min}$; $t \mathrm{R}: 12.77 \mathrm{~min}$ (minor enantiomer), $14.65 \mathrm{~min}$ (major enantiomer); ee (major enantiomer) $=93 \%$ (7c prepared by general procedure $\mathrm{E})$.
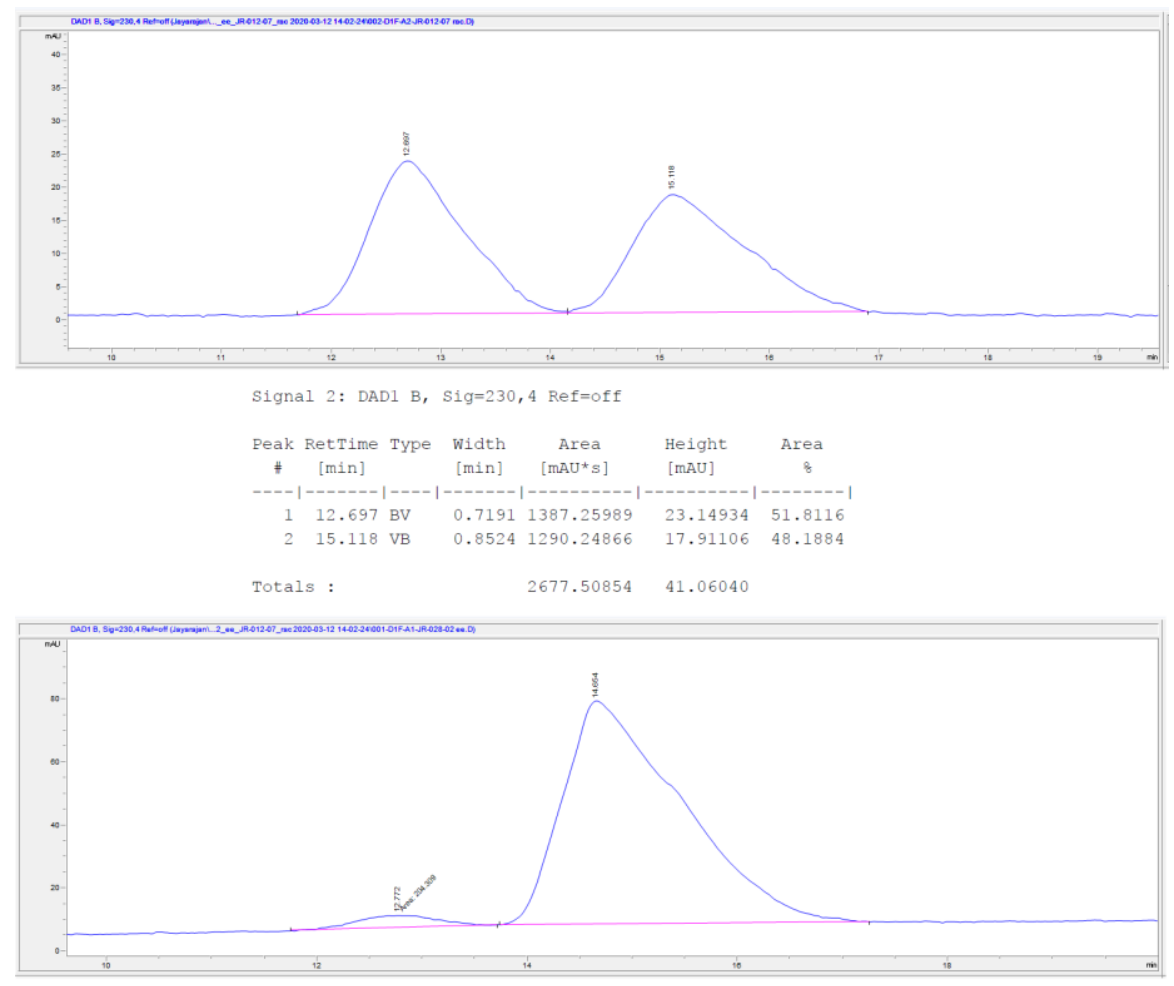

Signal 2: DADI B, Sig=230,4 Ref=off

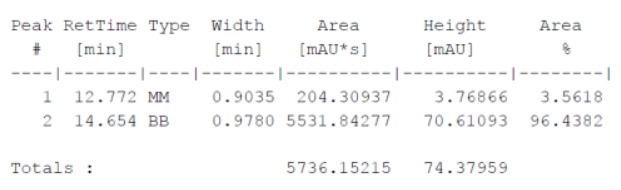




\section{8 Determination of the absolute configuration: $\operatorname{Mosher}{ }^{1} \mathrm{H}$ NMR method}

The absolute stereochemistry of product $\mathbf{7 c}$ was determined using the Mosher ${ }^{1} \mathrm{H}$ NMR method according to a literature procedure. ${ }^{4}$ Alcohol $7 \mathbf{c}$ was converted to the corresponding (S)-MPTA ester using $(R)$-MPTA-Cl. In the same way, the $(R)$-MPTA ester was obtained from $7 \mathbf{c}$ and $(S)$ MPTA-Cl (Figure S4).<smiles>OC(c1ccc(Br)cc1)[C@H](C=CC(F)(F)F)c1ccccc1</smiles>

7c<smiles>O[C@H](c1ccc(Br)cc1)[C@@H](/C=C/C(F)(F)F)c1ccccc1</smiles>

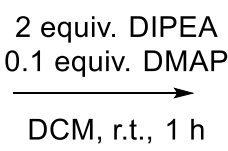

(R)-MPTA-Cl

2 equiv. DIPEA 0.1 equiv. DMAP DCM, r.t., $1 \mathrm{~h}$

(S)-MPTA-CI<smiles>[R][C@@](OC)(C(=O)O[C@H](c1ccc(Br)cc1)[C@H](/C=C/C(F)(F)F)c1ccccc1)c1ccccc1</smiles>

Figure S4. Derivatisation of $\mathbf{7 c}$ for determination of the absolute configuration by the Moshermethod.

The difference in ${ }^{1} \mathrm{H}$ NMR shift between the $(S)$-MPTA ester and the $(R)$-MPTA ester was used to determine the absolute configuration at the stereogenic tertiary carbon in alcohol 7c. Figure S5 shows the chemical shifts that were found for the $(S)$-MPTA ester and the $(R)$-MPTA ester. The difference between these shifts $\Delta \delta^{\mathrm{SR}}$ is calculated by $\delta(\mathrm{S})-\delta(\mathrm{R})$. As is shown, $\Delta \delta^{\mathrm{SR}}$ has the opposite sign for the two enantiotopic groups on the stereogenic tertiary carbon.
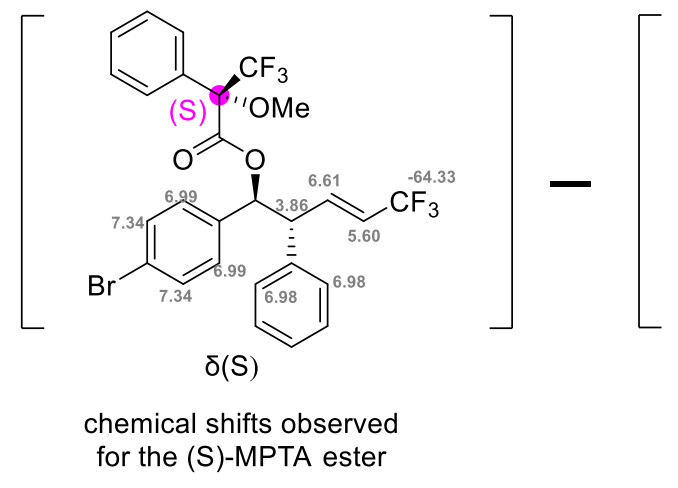

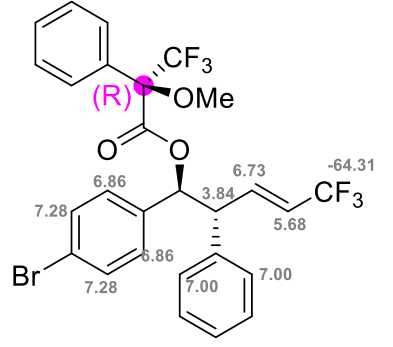

$\delta(R)$

chemical shifts observed for the (R)-MPTA ester

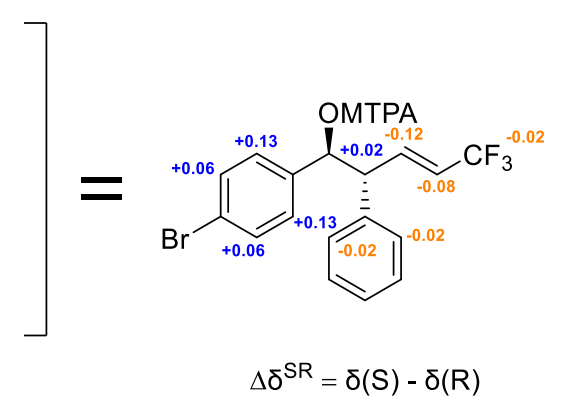

difference in chemical shift

Figure S5. Differences of the chemical shifts between the two diastereomeric derivatives of 7c. The sign of the difference $\Delta \delta^{\mathrm{SR}}$ is diagnostic for the absolute configuration of the carbon atom. 
In Figure S6 a simplified model is shown for alcohol 7c, in which the enantiotopic groups are represented by $\mathrm{R}^{1}$ (blue) and $\mathrm{R}^{2}$ (orange). According to the above mentioned (Figure $\mathrm{S} 4$ ) changes of the shift values, the C-OH carbon in $7 \mathbf{c}$ has an S-configuration, since the $\Delta \delta^{\mathrm{SR}}$ values are positive for $\mathrm{R}^{1}$. The reason is the anisotropic shielding of $\mathrm{R}^{1}$ by the phenyl group in $7 \mathbf{c}-(\mathbf{R})$-MPTA. Likewise, $\Delta \delta^{\mathrm{SR}}$ will be negative for $\mathrm{R}^{2}$, as it is shielded by the phenyl group in 7c-(S)-MPTA. In conclusion, the absolute configuration at the stereogenic tertiary carbon in product $\mathbf{7 c}$ can be assigned as $S$.

N.B. According to the C.I.P. convention the configuration label (R, S) of the corresponding stereocenters (magenta dot) in (R)-MPTA-Cl and 7c-(S)-MPTA is different, despite the fact that the absolute configuration of this carbon is the same.

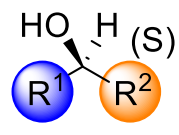

$7 c$<smiles>COC(C(=O)Cl)(c1ccccc1)C(F)(F)F</smiles>

(S)-MPTA-Cl
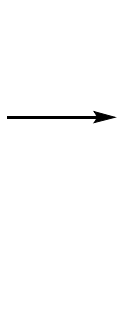

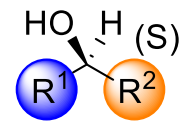

7c<smiles>COC(C(=O)Cl)(c1ccccc1)C(F)(F)F</smiles>

(R)-MPTA-Cl

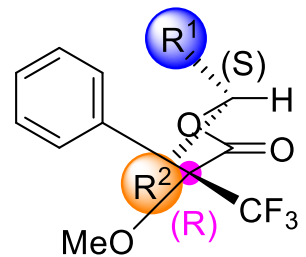

7c-(R)-MPTA

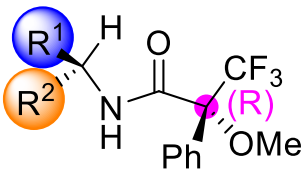

(R)-MPTA ester: $R^{1}$ is shielded by the magnetic anisotropy of the Ph group

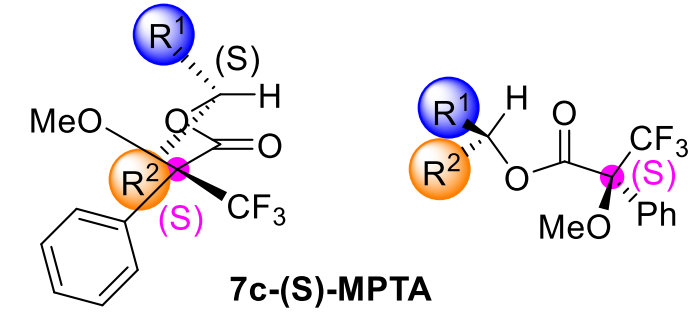

(S)-MPTA ester: $R^{2}$ is shielded by by the magnetic anisotropy of the Ph group

For $\mathbf{R}^{1}$ the $\delta(\mathrm{ppm})$ will be higher in the (S)-MPTA ester

For $\mathrm{R}^{2}$ the $\delta(\mathrm{ppm})$ will be higher in the (R)-MPTA ester

$\Delta \delta=\delta(S)-\delta(R)$

For $\mathrm{R}^{1}, \Delta \delta>0$ (positive)

For $\mathrm{R}^{2}, \Delta \delta<0$ (negative)

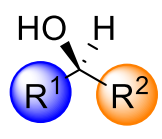

Figure S6. Explanation of the different sign of $\Delta \delta^{\mathrm{SR}}$ in the two diastereomers of 7c-MPTA. 
(1S,2R,E)-1-(4-bromophenyl)-5,5,5-trifluoro-2-(4-(trifluoromethyl)phenyl) pent-3-en-1-ol<smiles>O[C@H](c1ccc(Br)cc1)[C@H](/C=C/C(F)(F)F)c1ccc(C(F)(F)F)cc1</smiles>

(7d): This compound was obtained according to the above general procedure $\mathrm{C}$ using $30 \mathrm{~mol} \%$ of iodo-BINOL $4(0.03 \mathrm{mmol})$ at $30{ }^{\circ} \mathrm{C}$ for $48 \mathrm{~h}$ in step 1 and $40{ }^{\circ} \mathrm{C}$ for 4 hours in step 2. Product 7d was isolated in $42 \%$ yield $(18.4 \mathrm{mg}, 42 \mu \mathrm{mol})$ as colorless oil by silica gel chromatography using petroleum ether/ethyl acetate 40:1 as eluent.

${ }^{1} \mathbf{H}$ NMR (400 MHz, CDCl 3$) \delta 7.54(\mathrm{~d}, J=7.9 \mathrm{~Hz}, 2 \mathrm{H}), 7.44-7.36$ $(\mathrm{m}, 2 \mathrm{H}), 7.23(\mathrm{~d}, J=8.1 \mathrm{~Hz}, 2 \mathrm{H}), 7.10-6.99(\mathrm{~m}, 2 \mathrm{H}), 6.82(\mathrm{ddq}, J=$ 15.8, 8.1, 2.1 Hz, 1H), $5.60(\mathrm{dqd}, J=15.9,6.2,1.2 \mathrm{~Hz}, 1 \mathrm{H}), 4.94(\mathrm{dd}, J=6.8,2.6 \mathrm{~Hz}, 1 \mathrm{H}), 3.68$ $(\mathrm{ddd}, J=8.4,6.8,1.7 \mathrm{~Hz}, 1 \mathrm{H}), 2.12(\mathrm{~d}, J=3.0 \mathrm{~Hz}, 1 \mathrm{H}) ;{ }^{13} \mathbf{C} \mathbf{N M R}\left(\mathbf{1 0 1} \mathbf{M H z}, \mathbf{C D C l}_{3}\right) \boldsymbol{\delta} 142.90$, $140.43,138.24\left(\mathrm{q},{ }^{3} J_{\mathrm{C}-\mathrm{F}}=6.6 \mathrm{~Hz}\right), 131.71,129.92\left(\mathrm{q},{ }^{2} J_{\mathrm{C}-\mathrm{F}}=32.5 \mathrm{~Hz}\right), 129.03,128.13,125.86(\mathrm{q}$, $\left.{ }^{3} J_{\mathrm{C}-\mathrm{F}}=3.7 \mathrm{~Hz}\right), 122.01\left(\mathrm{q},{ }^{2} J_{\mathrm{C}-\mathrm{F}}=33.7 \mathrm{~Hz}\right), 122.74\left(\mathrm{q},{ }^{1} J_{\mathrm{C}-\mathrm{F}}=269.7 \mathrm{~Hz}\right), 122.71\left(\mathrm{q},{ }^{1} J_{\mathrm{C}-\mathrm{F}}=272.1\right.$ $\mathrm{Hz}), 76.63,55.71 ;{ }^{19} \mathbf{F}$ NMR (377 MHz, $\left.\mathbf{C D C l}_{3}\right) \boldsymbol{\delta}-62.61,-64.16(\mathrm{dt}, J=6.6,2.2 \mathrm{~Hz}) . \mathbf{H R M S}$ (neg. ESI) $\mathbf{m} / \mathbf{z}$ : calcd for $\mathrm{C}_{18} \mathrm{H}_{12} \mathrm{BrF}_{6} \mathrm{O}[\mathrm{M}-\mathrm{H}]^{-}$436.9981, Found 436.9986. $[\boldsymbol{\alpha}]_{\boldsymbol{D}}^{\mathbf{2 5}}=+7.73(c$ $\left.0.375, \mathrm{CHCl}_{3}\right)$.

Determination of $\boldsymbol{e} e$ by Chiral SFC: Diacel ID, 35 bar, $40{ }^{\circ} \mathrm{C}, 0.46 \mathrm{~cm} \varphi, 25 \mathrm{~cm}$ column, $5 \%$ $\mathrm{MeOH}$ in $\mathrm{CO}_{2}$, flow rate: $2.0 \mathrm{~mL} / \mathrm{min}$; $t \mathrm{R}$ : $5.08 \mathrm{~min}$ (minor enantiomer), 6.21 min (major enantiomer); ee (major enantiomer) $=90 \%$.
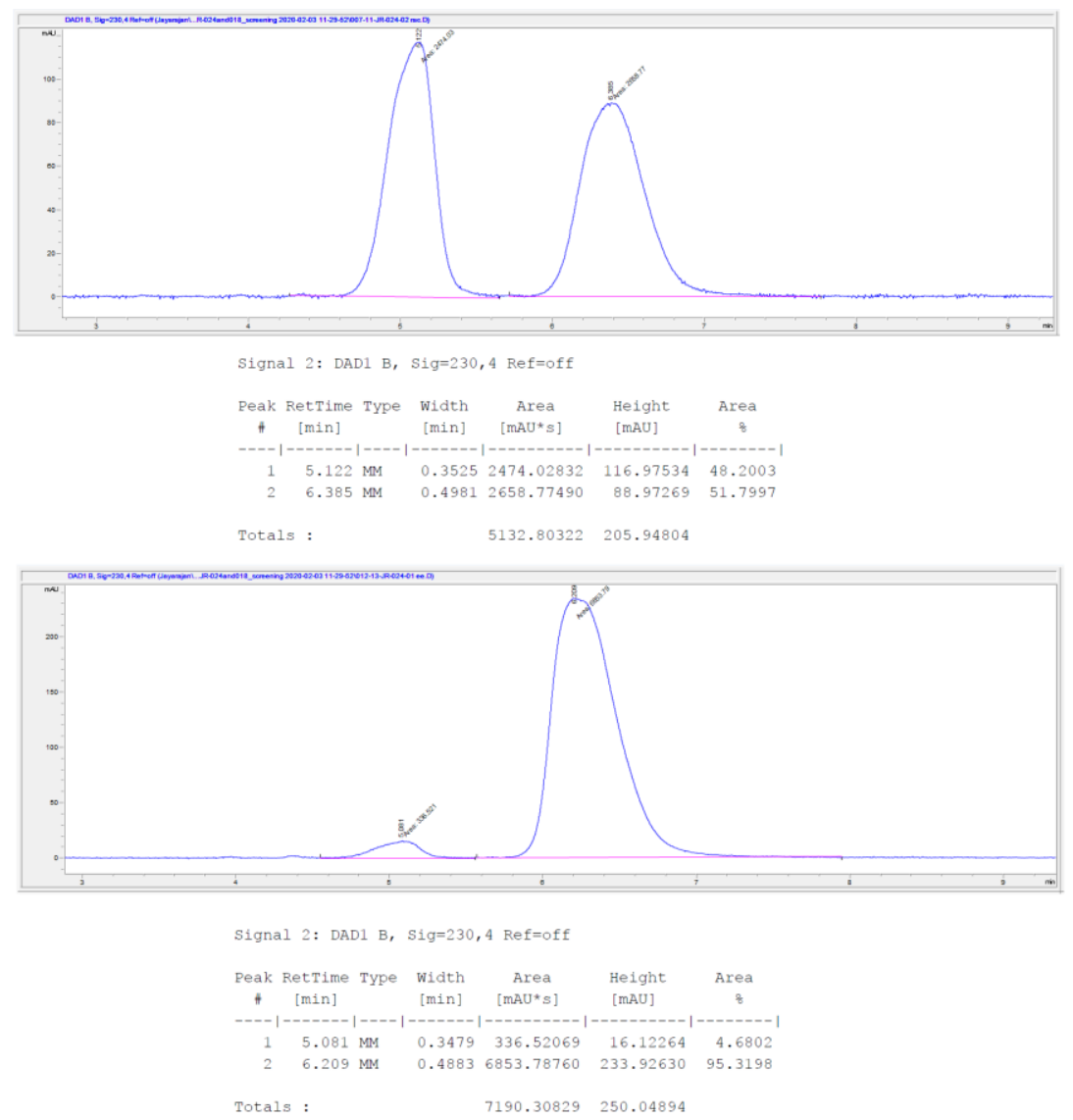
(1S,2R,E)-1-(4-bromophenyl)-2-(4-chlorophenyl)-5,5,5-trifluoropent-3-en-1-ol (7e): This<smiles>OC(c1ccc(Br)cc1)C(/C=C/C(F)(F)F)c1ccc(Cl)cc1</smiles>
compound was obtained according to the above general procedure $\mathrm{C}$ using $30 \mathrm{~mol} \%$ of iodo-BINOL $4(0.03 \mathrm{mmol})$. Product $7 \mathbf{e}$ was isolated in $48 \%$ yield $(19.6 \mathrm{mg}, 48 \mu \mathrm{mol})$ as colourless oil by silica gel chromatography using pentane/ethyl acetate $35: 1$ as eluent.

${ }^{1} \mathbf{H}$ NMR (400 MHz, $\left.\mathbf{C D C l}_{3}\right) \boldsymbol{\delta} 7.43-7.34(\mathrm{~m}, 2 \mathrm{H}), 7.26-7.19(\mathrm{~m}$, $2 \mathrm{H}$ ), 7.02 (ddt, $J=6.6,5.1,2.2 \mathrm{~Hz}, 4 \mathrm{H}$ ), 6.79 (ddq, $J=15.8,8.0,2.1$ $\mathrm{Hz}, 1 \mathrm{H}), 5.60(\mathrm{dqd}, J=15.9,6.2,1.3 \mathrm{~Hz}, 1 \mathrm{H}), 4.88(\mathrm{~d}, J=7.0 \mathrm{~Hz}$, $1 \mathrm{H}), 3.60(\mathrm{ddt}, J=8.5,7.2,1.7 \mathrm{~Hz}, 1 \mathrm{H}), 2.11(\mathrm{~s}, 1 \mathrm{H}) ;{ }^{13} \mathbf{C} \mathbf{~ N M R}(\mathbf{1 0 1}$ MHz, $\left.\mathbf{C D C l}_{3}\right) \delta$ 140.57, $138.76\left(\mathrm{q},{ }^{3} J_{C-F}=6.5 \mathrm{~Hz}\right), 137.19,133.47,131.63,129.94,129.10$, $128.18,122.83\left(\mathrm{q},{ }^{1} J_{C-F}=269.8 \mathrm{~Hz}\right), 122.11,121.63\left(\mathrm{q},{ }^{2} J_{C-F}=33.6 \mathrm{~Hz}\right), 76.72,55.31 ;{ }^{19} \mathbf{F}$ NMR $\left(377 \mathrm{MHz}, \mathbf{C D C l}_{3}\right) \delta-64.07(\mathrm{dt}, J=6.2,1.7 \mathrm{~Hz}$ ). HRMS (neg. ESI) $\mathbf{m} / \mathbf{z}$ : calcd for $\mathrm{C}_{18} \mathrm{H}_{14} \mathrm{BrClF}_{3} \mathrm{O}_{3}\left[\mathrm{M}-\mathrm{H}+\mathrm{CH}_{2} \mathrm{O}_{2}\right]^{-}$448.9772. Found 448.9759. $[\alpha]_{D}^{26}=+19.6\left(c 0.25, \mathrm{CHCl}_{3}\right)$.

Determination of $e \boldsymbol{e}$ by Chiral SFC: Daicel CHIRALPAK IA- $3,25^{\circ} \mathrm{C}, 0.3 \mathrm{~cm} \phi, 15 \mathrm{~cm}$ column, $5 \% \mathrm{MeOH}$ in $\mathrm{CO}_{2}$, flow rate: $0.8 \mathrm{~mL} / \mathrm{min}$; $t \mathrm{R}$ : 6.37 min (minor enantiomer), 9.20 min (major enantiomer); $e e($ major enantiomer $)=75 \%$.

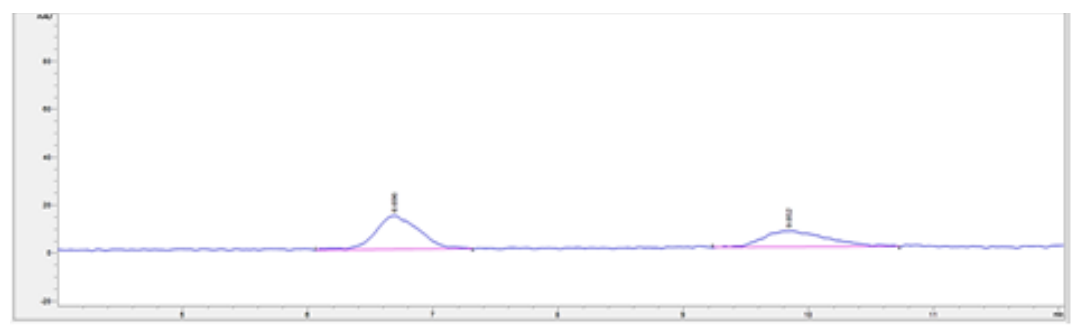

Signal 2: DAD1 B, Sig=230,4 Ref=off
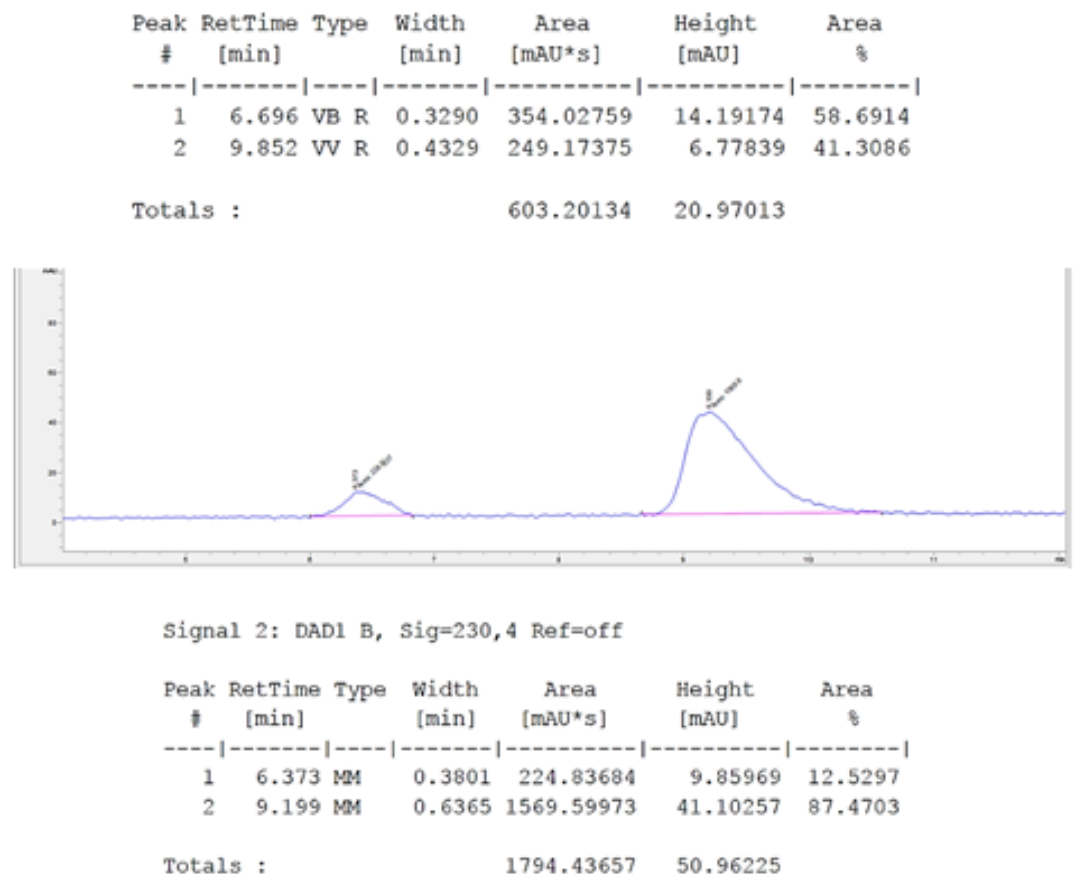
(2S,3S)-2-(4-bromophenyl)-3-(3,3,3-trifluoropropyl)nonan-2-ol (7f): This compound was<smiles>CCCCCC[C@H](/C=C/C(F)(F)F)[C@](C)(O)c1ccc(Br)cc1</smiles>
obtained according to the above general procedure E. Product $7 \mathbf{f}$ was isolated in $67 \%$ yield $(26.3 \mathrm{mg}, 67 \mu \mathrm{mol})$ as a colorless oil by silica gel chromatography using pentane/ethyl acetate 20:1 as eluent.

${ }^{1} \mathbf{H}$ NMR $\left(400 \mathrm{MHz}, \mathbf{C D C l}_{3}\right) \boldsymbol{\delta} 7.50(\mathrm{~d}, J=8.6 \mathrm{~Hz}, 2 \mathrm{H}), 7.28(\mathrm{~d}, J=8.7 \mathrm{~Hz}, 2 \mathrm{H}), 6.12(\mathrm{ddq}, J=$ $16.1,9.9,2.1 \mathrm{~Hz}, 1 \mathrm{H}), 5.57(\mathrm{dqd}, J=15.8,6.3,0.7 \mathrm{~Hz}, 1 \mathrm{H}), 2.34(\mathrm{t}, J=9.8 \mathrm{~Hz}, 1 \mathrm{H}), 1.74(\mathrm{~s}, 1 \mathrm{H})$, $1.60(\mathrm{~s}, 3 \mathrm{H}), 1.34-1.00(\mathrm{~m}, 10 \mathrm{H}), 0.88(\mathrm{t}, J=7.0 \mathrm{~Hz}, 3 \mathrm{H}) ;{ }^{13} \mathbf{C}$ NMR $\left(\mathbf{1 0 1} \mathbf{M H z}, \mathbf{C D C l}_{3}\right) \delta$ $145.05,140.56\left(\mathrm{q},{ }^{3} J_{C-F}=6.4 \mathrm{~Hz}\right), 131.27,127.57,125.40\left(\mathrm{q},{ }^{1} J_{C-F}=269.7 \mathrm{~Hz}\right), 121.49\left(\mathrm{q},{ }^{2} J_{C-F}=\right.$ $33.3 \mathrm{~Hz}), 121.29,75.72,75.71,54.12,31.76,29.23,28.30,27.68,26.93,22.71,14.16 ;{ }^{19} \mathbf{F}$ NMR (377 MHz, $\mathbf{C D C l}_{3}$ ) $\delta$-63.53 - -64.71 (m). HRMS (neg. ESI) m/z: calcd for $\mathrm{C}_{19} \mathrm{H}_{25} \mathrm{BrF}_{3} \mathrm{O}_{3}$ $\left[\mathrm{M}-\mathrm{H}+\mathrm{CH}_{2} \mathrm{O}_{2}\right]^{-} 437.0945$. Found 437.0974. $[\boldsymbol{\alpha}]_{D}^{24}=-22.0\left(c 0.44, \mathrm{CHCl}_{3}\right)$.

Determination of $e \boldsymbol{e}$ by Chiral SFC: Diacel CHIRALCEL OJ-H, $25^{\circ} \mathrm{C}, 0.3 \mathrm{~cm} \phi, 15 \mathrm{~cm}$ column, $0.5 \% \mathrm{MeOH}$ in $\mathrm{CO}_{2}$, flow rate: $1.6 \mathrm{~mL} / \mathrm{min}$; $t \mathrm{R}: 2.62 \mathrm{~min}$ (minor enantiomer), 3.68 min (major enantiomer); ee (major enantiomer) $=98 \%$.

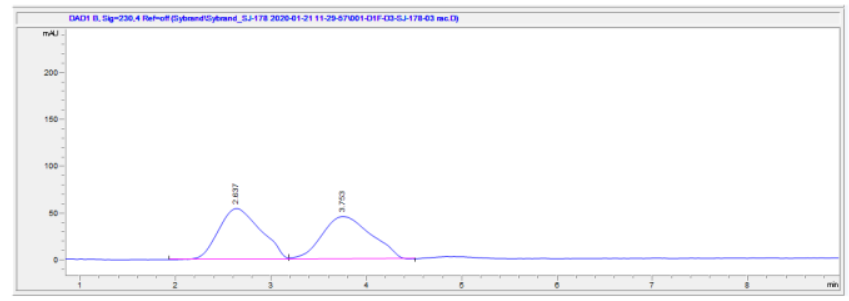

Signal 2: DAD1 B, Sig=230,4 Ref=off

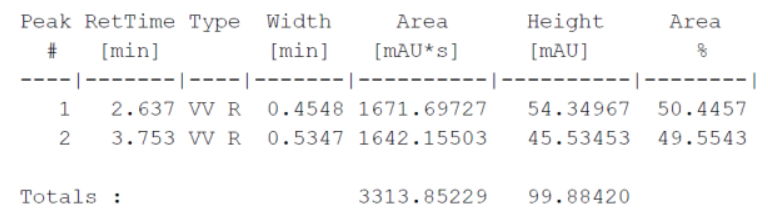

Totals :

$3313.85229 \quad 99.88420$

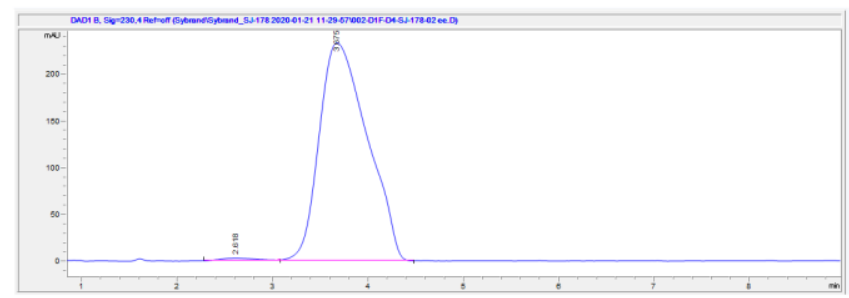

Signal 2: DAD1 B, Sig=230,4 Ref=off

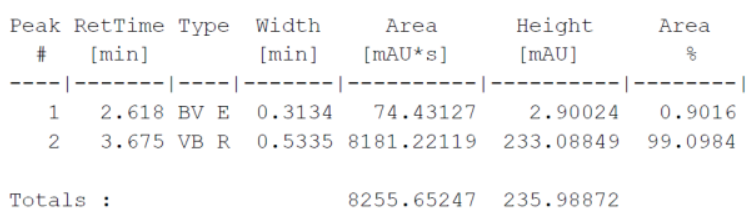


(R,E)-1-(4,4,4-trifluoro-1-phenylbut-2-en-1-yl)cyclohexan-1-ol (7g) This compound was<smiles>OC1([C](/C=C/C(F)(F)F)c2ccccc2)CCCC1</smiles>
obtained according to the above general procedure E. Product $\mathbf{7 g}$ was isolated in $72 \%$ yield $(20.4 \mathrm{mg}, 72 \mu \mathrm{mol})$ as a colorless oil by silica gel chromatography using DCM/pentane 1:4 as eluent. ${ }^{1} \mathbf{H}$ NMR (400 MHz, $\left.\mathbf{C D C l}_{3}\right) \boldsymbol{\delta} 7.39-7.31(\mathrm{~m}, 2 \mathrm{H}), 7.31-7.21(\mathrm{~m}, 3 \mathrm{H}), 6.90$ (ddq, $J=16.0$, 9.8, $2.2 \mathrm{~Hz}, 1 \mathrm{H}), 5.67(\mathrm{dq}, J=15.8,6.3 \mathrm{~Hz}, 1 \mathrm{H}), 3.32(\mathrm{~d}, J=9.8 \mathrm{~Hz}, 1 \mathrm{H})$, $1.72-1.64(\mathrm{~m}, 1 \mathrm{H}), 1.63-1.53(\mathrm{~m}, 3 \mathrm{H}), 1.53-1.46(\mathrm{~m}, 2 \mathrm{H}), 1.43-1.14$ $(\mathrm{m}, 5 \mathrm{H}) ;{ }^{13} \mathbf{C}$ NMR $\left(\mathbf{1 0 1} \mathbf{M H z}, \mathbf{C D C l}_{3}\right) \delta 139.91(\mathrm{q}, J=6.5 \mathrm{~Hz}), 139.01,129.37,128.71,127.37$, $123.03(\mathrm{q}, J=269.6 \mathrm{~Hz}), 120.53(\mathrm{q}, J=33.3 \mathrm{~Hz}), 72.77,58.59$, 36.33, 35.92, 25.57, 21.88, 21.86; ${ }^{19} \mathbf{F}$ NMR $\left(377 \mathrm{MHz}, \mathbf{C D C l}_{3}\right) \delta-63.81(\mathrm{dd}, J=6.2,2.0 \mathrm{~Hz})$; After repeated attempts we were unable to obtain HRMS data for compound $\mathbf{7 g}$, probably due to its resistance to ionization. GCMS m/z: $99.10(100.00 \%), 81.05$ (74.55\%), 166.00 (11.95\%), $115.05(8.44 \%) ;[\boldsymbol{\alpha}]_{D}^{27}=-24.8(c$ $\left.0.46, \mathrm{CHCl}_{3}\right)$.

Determination of $e \boldsymbol{e}$ by Chiral SFC: Diacel CHIRALPAK IA-3, $25^{\circ} \mathrm{C}, 0.3 \mathrm{~cm} \phi, 15 \mathrm{~cm}$ column, $0.5 \% \mathrm{MeOH}$ in $\mathrm{CO}_{2}$, flow rate: $1.0 \mathrm{~mL} / \mathrm{min}$; $t \mathrm{R}: 6.76 \mathrm{~min}$ (major enantiomer), $9.01 \mathrm{~min}$ (minor enantiomer); $e e($ major enantiomer $)=91 \%$.

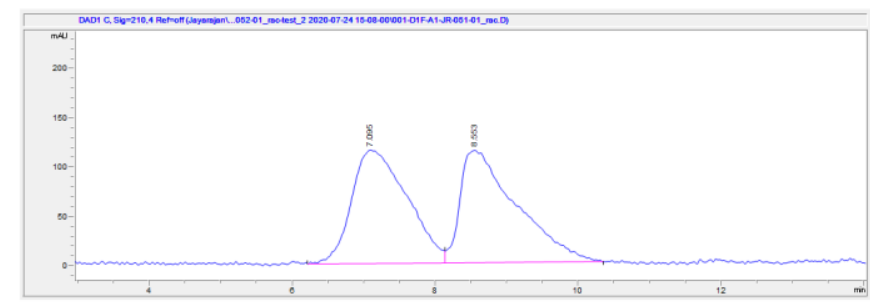

Signal 3: DAD1 C, Sig=210,4 Ref=off
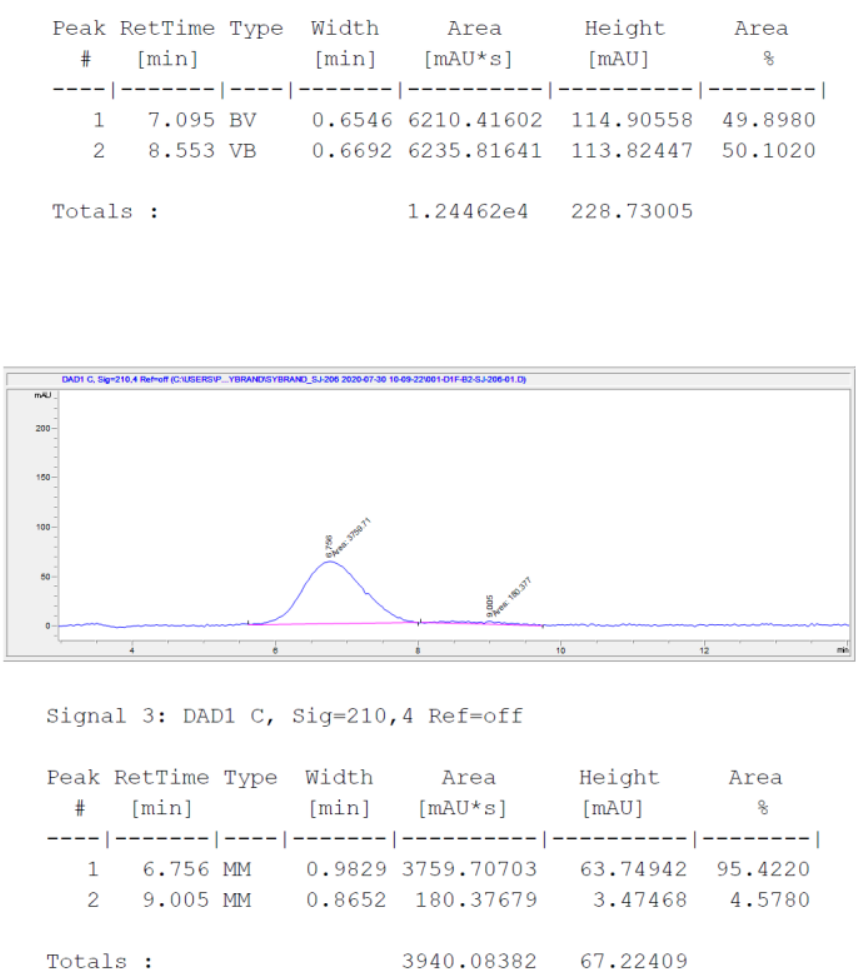
(1R,2S)-2-methyl-1-((R,E)-4,4,4-trifluoro-1-phenylbut-2-en-1-yl)cyclohexan-1-ol (7h) This<smiles>OC1(/C=C/C(F)(F)F)[C@H]2CCC[C@@H]1CCC2</smiles>
compound was obtained according to the above general procedure E, except 1 equiv $(0.1 \mathrm{mmol})$ of $\mathbf{6} \mathbf{d}$ was used. Product $\mathbf{7 h}$ was isolated in $50 \%$ yield $(14.8 \mathrm{mg}, 50 \mu \mathrm{mol})$ as a colorless oil by silica gel chromatography using DCM/pentane 1:4 as eluent. ${ }^{\mathbf{1}} \mathbf{H}$ NMR (400 $\left.\mathbf{M H z}, \mathbf{C D C l}_{3}\right) \boldsymbol{\delta} 7.37-$ $7.31(\mathrm{~m}, 2 \mathrm{H}), 7.31-7.25(\mathrm{~m}, 3 \mathrm{H}), 6.94(\mathrm{ddq}, J=15.8,8.0,2.2 \mathrm{~Hz}, 1 \mathrm{H})$, $5.59(\mathrm{dqd}, J=15.8,6.3,1.4 \mathrm{~Hz}, 1 \mathrm{H}), 3.80(\mathrm{dt}, J=8.0,1.9 \mathrm{~Hz}, 1 \mathrm{H}), 1.72-$ $1.61(\mathrm{~m}, 1 \mathrm{H}), 1.59-1.32(\mathrm{~m}, 8 \mathrm{H}), 1.19-1.07(\mathrm{~m}, 1 \mathrm{H}), 1.00(\mathrm{~d}, J=6.1 \mathrm{~Hz}, 3 \mathrm{H}) ;{ }^{13} \mathrm{C}$ NMR $(101$ $\left.\mathrm{MHz} \mathrm{CDCl}_{3}\right) \delta 140.65(\mathrm{q}, J=6.4 \mathrm{~Hz}), 138.84,129.49,128.54,127.19,123.04(\mathrm{q}, J=269.5 \mathrm{~Hz})$, $120.55(\mathrm{q}, J=33.2 \mathrm{~Hz}), 75.52,54.92,35.82,32.51,30.65,24.07,21.72,15.13 ;{ }^{19} \mathbf{F}$ NMR $(377$ $\mathrm{MHz}, \mathrm{CDCl}_{3}$ ) $\delta-63.66\left(\mathrm{dt}, J=5.3,3.2,2.4 \mathrm{~Hz}\right.$ ); HRMS (neg. ESI) m/z: calcd for $\mathrm{C}_{18} \mathrm{H}_{22} \mathrm{~F}_{3} \mathrm{O}_{3}$ $\left[\mathrm{M}+\mathrm{HCO}_{2}\right]^{-} 343.1527$. Found 343.1522; $[\boldsymbol{\alpha}]_{D}^{26}=-46.2\left(c 0.27, \mathrm{CHCl}_{3}\right)$.

Determination of $e \boldsymbol{e}$ by Chiral SFC: Diacel CHIRALPAK IA, $40{ }^{\circ} \mathrm{C}, 0.46 \mathrm{~cm} \phi, 25 \mathrm{~cm}$ column, $4 \% \mathrm{MeOH}$ in $\mathrm{CO}_{2}$, flow rate: $2.0 \mathrm{~mL} / \mathrm{min}$; $t \mathrm{R}: 6.48 \mathrm{~min}$ (major enantiomer), 8.36 min (major enantiomer); ee (major enantiomer) $=97 \%$.
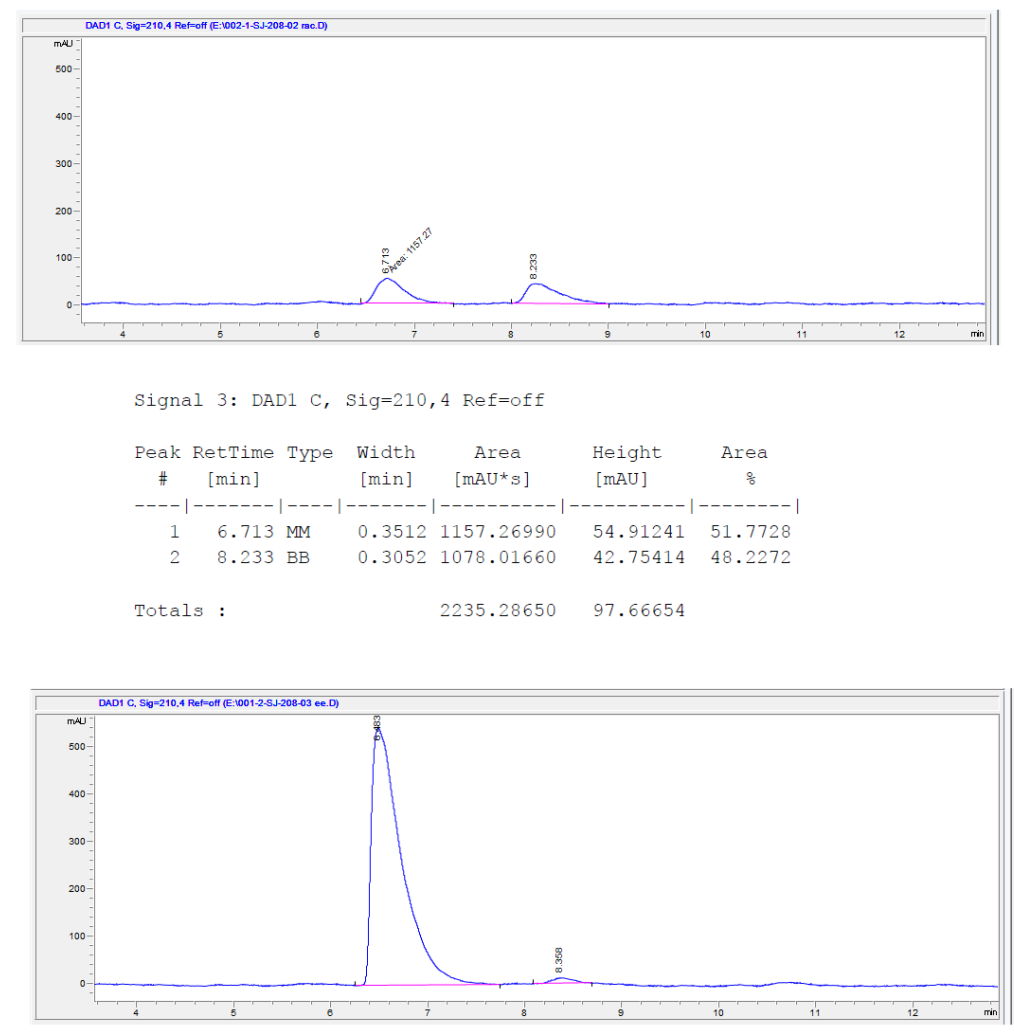

Signal 3: DAD1 C, Sig=210,4 Ref=off

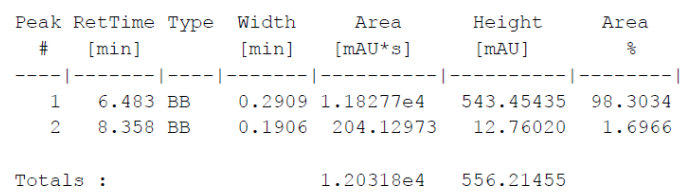


Ethyl (2S,3R,E)-2-(bromomethyl)-6,6,6-trifluoro-2-hydroxy-3-phenylhex-4-enoate (7i) This<smiles>CCOC(=O)C(Br)(Br)[C@@H](/C=C/C(F)(F)F)c1ccccc1</smiles>
compound was obtained according to the above general procedure E. Product $7 \mathbf{i}$ was isolated in $72 \%$ yield $(27.4 \mathrm{mg}, 72 \mu \mathrm{mol})$ as a colorless solid by silica gel chromatography using DCM/pentane 1:4 as eluent. The relative stereochemistry was assigned based on previous reports of similar reactions ${ }^{5}$. Mp: $53.0{ }^{\circ} \mathrm{C} .{ }^{1} \mathbf{H}$ NMR $\left(400 \mathbf{~ M H z}, \mathbf{C D C l}_{3}\right) \delta 7.40-$ $7.29(\mathrm{~m}, 5 \mathrm{H}), 6.72(\mathrm{ddq}, J=15.6,9.3,2.1 \mathrm{~Hz}, 1 \mathrm{H}), 5.65(\mathrm{dqd}, J=15.7$, $6.3,0.9 \mathrm{~Hz}, 1 \mathrm{H}), 4.35(\mathrm{q}, J=7.1 \mathrm{~Hz}, 2 \mathrm{H}), 3.81(\mathrm{dd}, J=9.2,1.2 \mathrm{~Hz}, 1 \mathrm{H}), 3.75(\mathrm{~d}, J=0.8 \mathrm{~Hz}, 1 \mathrm{H})$, $3.56(\mathrm{~d}, J=10.4 \mathrm{~Hz}, 1 \mathrm{H}), 3.11(\mathrm{~d}, J=10.5 \mathrm{~Hz}, 1 \mathrm{H}), 1.37(\mathrm{t}, J=7.1 \mathrm{~Hz}, 3 \mathrm{H}) ;{ }^{13} \mathbf{C}$ NMR $(\mathbf{1 0 1} \mathbf{M H z}$, $\left.\mathbf{C D C l}_{3}\right) \delta 172.45,138.06(\mathrm{q}, J=6.5 \mathrm{~Hz}), 135.79,129.52,129.06,128.39,122.56(\mathrm{q}, J=269.7$ $\mathrm{Hz}), 121.23$ (q, $J=34.0 \mathrm{~Hz}), 79.37,63.43,54.22,38.32,14.37 ;{ }^{19} \mathbf{F} \mathbf{N M R}\left(\mathbf{3 7 7} \mathbf{~ M H z}, \mathbf{C D C l}_{3}\right) \boldsymbol{\delta}$ $64.41\left(\mathrm{~d}, J=5.9 \mathrm{~Hz}\right.$ ); HRMS (pos. ESI) m/z: calcd for $\mathrm{C}_{15} \mathrm{H}_{16} \mathrm{BrF}_{3} \mathrm{NaO}_{3}[\mathrm{M}+\mathrm{Na}]^{+} 403.0127$. Found 403.0134. $[\boldsymbol{\alpha}]_{D}^{27}=-14.6\left(c 0.59, \mathrm{CHCl}_{3}\right)$;

Determination of $\boldsymbol{e} \boldsymbol{e}$ by Chiral HPLC: Diacel CHIRALPAK AS-H, $25^{\circ} \mathrm{C}, 0.46 \mathrm{~cm} \phi, 25 \mathrm{~cm}$ column, $5 \%{ }^{\mathrm{i}} \mathrm{PrOH}$ in hexane, flow rate: $1.0 \mathrm{~mL} / \mathrm{min}$; $t \mathrm{R}: 4.73 \mathrm{~min}$ (minor enantiomer), $5.17 \mathrm{~min}$ (major enantiomer); ee (major enantiomer) $=82 \%$.
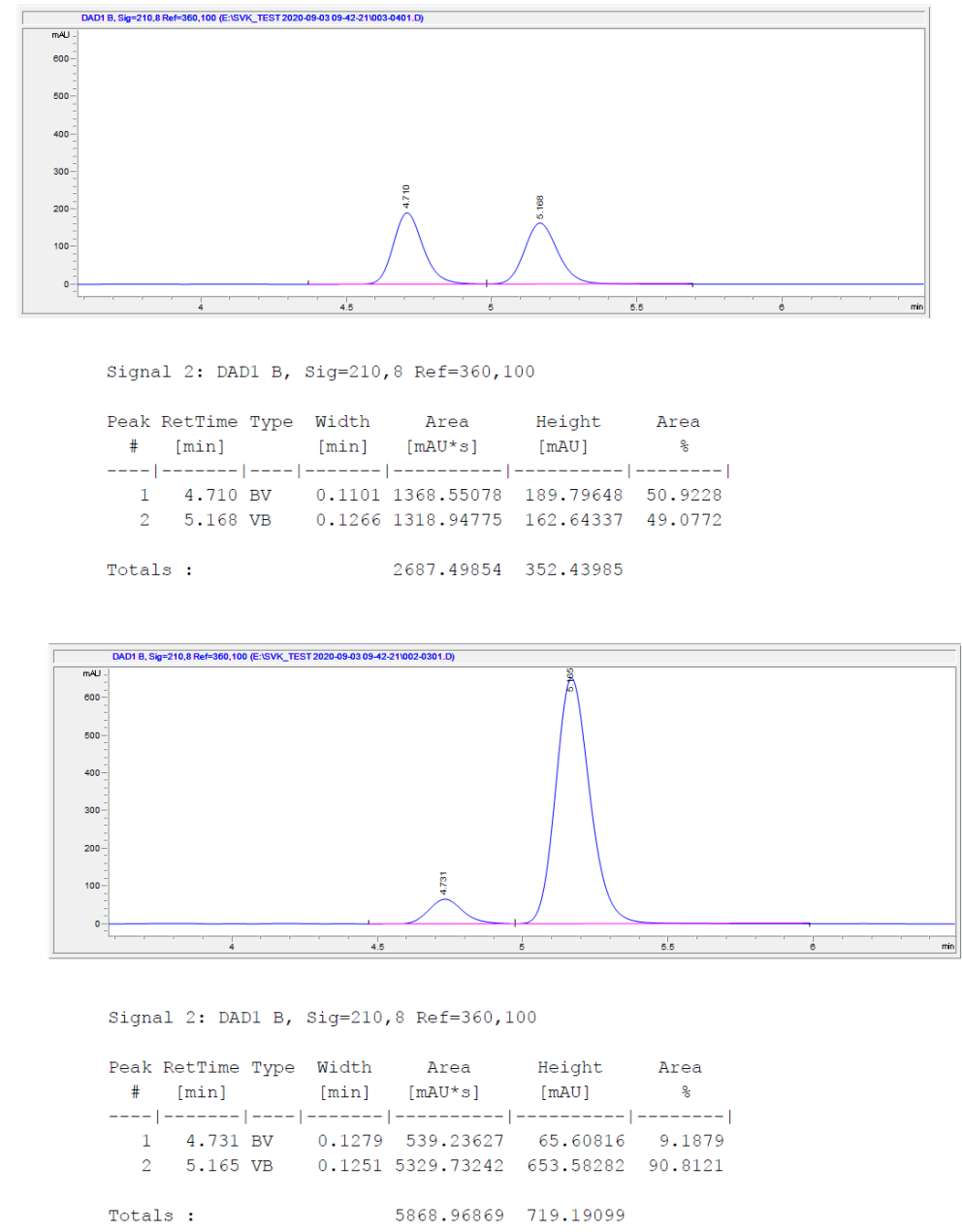
(R)-2-((R,E)-4,4,4-trifluoro-1-phenylbut-2-en-1-yl)indoline (7j): This compound was obtained<smiles>FC(F)(F)/C=C/[C@H](c1ccccc1)C1Cc2ccccc2N1</smiles>
according to the above general procedure E. Product $\mathbf{7 j}$ was isolated in $62 \%$ yield $(18.9 \mathrm{mg}, 62 \mu \mathrm{mol})$ as colorless viscous oil by silica gel chromatography using petroleum ether/ethyl acetate 20:1 as eluent.

${ }^{1} \mathbf{H}$ NMR (400 MHz, CDCl$) \boldsymbol{~} \boldsymbol{\delta} 7.44-7.35(\mathrm{~m}, 2 \mathrm{H}), 7.36-7.27(\mathrm{~m}$, $1 \mathrm{H}), 7.26-7.17(\mathrm{~m}, 2 \mathrm{H}), 7.10-6.94(\mathrm{~m}, 2 \mathrm{H}), 6.79-6.56(\mathrm{~m}, 3 \mathrm{H}), 5.76$ (dqd, $J=15.7,6.3,1.0 \mathrm{~Hz}, 1 \mathrm{H}), 4.20(\mathrm{q}, J=8.5 \mathrm{~Hz}, 1 \mathrm{H}), 4.01(\mathrm{~s}, 1 \mathrm{H})$, $3.50(\mathrm{tt}, J=8.8,1.4 \mathrm{~Hz}, 1 \mathrm{H}), 2.92(\mathrm{dd}, J=15.9,8.7 \mathrm{~Hz}, 1 \mathrm{H}), 2.70(\mathrm{ddt}$, $J=15.9,8.0,1.0 \mathrm{~Hz}, 1 \mathrm{H}) ;{ }^{13} \mathbf{C} \mathbf{N M R}\left(\mathbf{1 0 1} \mathbf{M H z}, \mathbf{C D C l}_{\mathbf{3}}\right) \boldsymbol{\delta} 150.08,140.81\left(\mathrm{q},{ }^{3} J_{C-F}=6.4 \mathrm{~Hz}\right)$, $139.64,129.27,128.14,128.05,127.69,127.60,124.85,122.88\left(\mathrm{q},{ }^{1} J_{C-F}=269.6 \mathrm{~Hz}\right), 120.62(\mathrm{q}$, $\left.{ }^{2} J_{C-F}=33.6 \mathrm{~Hz}\right), 119.27,109.51,63.02,54.17,34.76 ;{ }^{19} \mathbf{F} \mathbf{~ N M R}\left(377 \mathbf{~ M H z}, \mathbf{C D C l}_{3}\right) \boldsymbol{\delta}-63.82(\mathrm{~d}$, $\mathrm{J}=6.2 \mathrm{~Hz}$ ). HRMS (pos. ESI) m/z: calcd for $\mathrm{C}_{18} \mathrm{H}_{17} \mathrm{~F}_{3} \mathrm{~N}[\mathrm{M}+\mathrm{H}]^{+}$304.1308. Found 304.1304. $[\alpha]_{D}^{26}=-44.23\left(c 0.425, \mathrm{CHCl}_{3}\right)$.

Determination of $e \boldsymbol{e}$ by Chiral SFC: Diacel CHIRALCEL OJ-H, $25^{\circ} \mathrm{C}, 0.3 \mathrm{~cm} \phi, 15 \mathrm{~cm}$ column, $3 \% \mathrm{MeOH}$ in $\mathrm{CO}_{2}$, flow rate: $1.2 \mathrm{~mL} / \mathrm{min}$; $t \mathrm{R}$ : 6.99 min (minor enantiomer), 7.33 min (major enantiomer); $e e$ (major enantiomer) $=93 \%$.

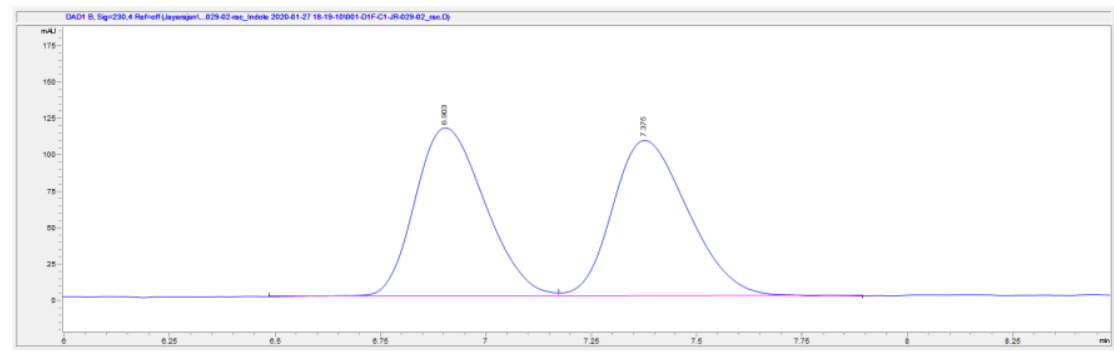

Signal 2: DAD1 B, Sig $=230,4$ Ref $=$ off
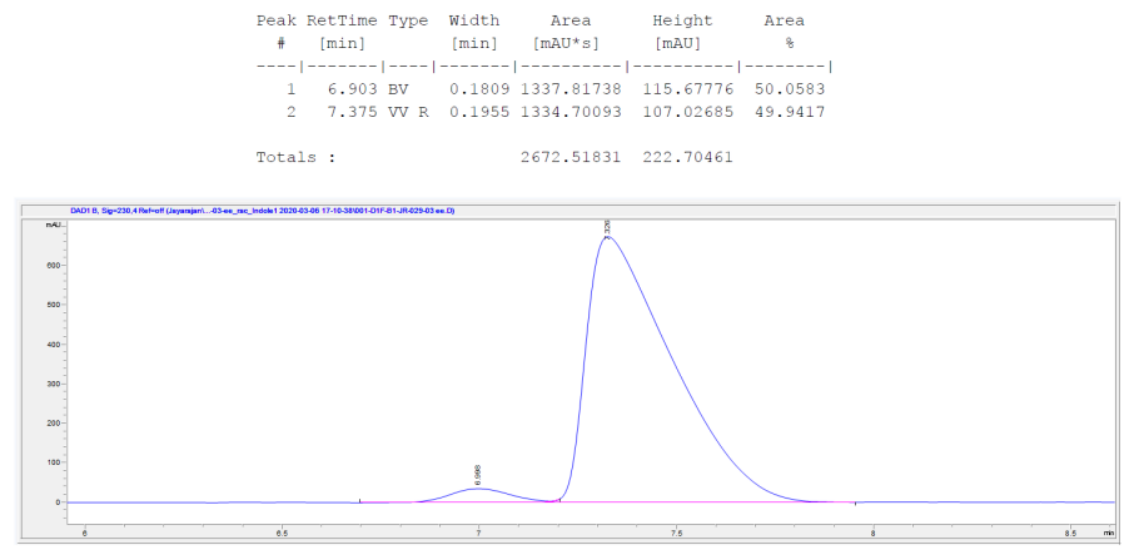

Signa1 2: DAD1 B, Sig=230,4 Ref $=$ off

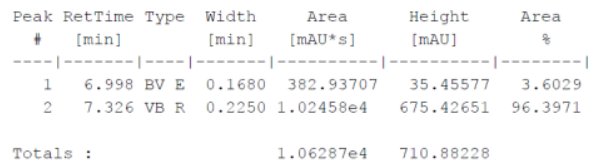


(R)-2-((R,E)-4,4,4-trifluoro-1-phenylbut-2-en-1-yl)indoline (7k): This compound was obtained<smiles>CC1c2ccccc2NC1[C](/C=C/C(F)(F)F)c1ccccc1</smiles>
according to the above general procedure E. Product $7 \mathbf{k}$ was isolated in $48 \%$ yield $(15.2 \mathrm{mg}, 48 \mu \mathrm{mol})$ as colorless viscous oil by silica gel chromatography using petroleum ether/ethyl acetate $20: 1$ as eluent.

${ }^{1} \mathbf{H}$ NMR (400 MHz, $\left.\mathbf{C D C l}_{3}\right) \boldsymbol{\delta} 7.43-7.36(\mathrm{~m}, 2 \mathrm{H}), 7.34-7.28(\mathrm{~m}$, $1 \mathrm{H}), 7.26-7.18(\mathrm{~m}, 2 \mathrm{H}), 7.04(\mathrm{tt}, J=7.6,1.1 \mathrm{~Hz}, 1 \mathrm{H}), 7.00(\mathrm{~d}, J=7.4$ $\mathrm{Hz}, 1 \mathrm{H}), 6.85-6.69(\mathrm{~m}, 2 \mathrm{H}), 6.62(\mathrm{dt}, J=7.8,0.7 \mathrm{~Hz}, 1 \mathrm{H}), 5.75(\mathrm{dqd}$, $J=15.8,6.2,1.0 \mathrm{~Hz}, 1 \mathrm{H}), 3.91(\mathrm{~s}, 1 \mathrm{H}), 3.72(\mathrm{t}, J=7.4 \mathrm{~Hz}, 1 \mathrm{H}), 3.50$ (ddt $J=8.9,7.4,1.4 \mathrm{~Hz}, 1 \mathrm{H}), 3.02(\mathrm{p}, J=6.9 \mathrm{~Hz}, 1 \mathrm{H}), 1.03(\mathrm{~d}, J=6.8$ $\mathrm{Hz}, 3 \mathrm{H}) ;{ }^{13} \mathbf{C}$ NMR $\left(126 \mathbf{~ M H z}, \mathbf{C D C l}_{3}\right) \boldsymbol{\delta} 149.34,140.47\left(\mathrm{q},{ }^{3} J_{\mathrm{C}-\mathrm{F}}=6.4 \mathrm{~Hz}\right), 139.61,133.34$, $129.21,128.28,127.78,127.58,123.77,122.91\left(\mathrm{q},{ }^{1} J_{\mathrm{C}-\mathrm{F}}=270.1 \mathrm{~Hz}\right), 120.70\left(\mathrm{q},{ }^{2} J_{\mathrm{C}-\mathrm{F}}=33.5 \mathrm{~Hz}\right)$, 118.99, 109.29, 70.96, 53.23, 41.08, 19.57; ${ }^{19} \mathbf{F}$ NMR (377 MHz, CDCl $) \boldsymbol{\delta}-63.89$ (m). HRMS (pos. ESI) m/z: calcd for $\mathrm{C}_{19} \mathrm{H}_{19} \mathrm{~F}_{3} \mathrm{~N}[\mathrm{M}+\mathrm{H}]^{+}$318.1464. Found 318.1466. $[\boldsymbol{\alpha}]_{\boldsymbol{D}}^{26}=-46.00(c \mathrm{cos}$, $\left.\mathrm{CHCl}_{3}\right)$.

Determination of $e$ by Chiral SFC: Diacel CHIRALCEL OJ-H, $25^{\circ} \mathrm{C}, 0.3 \mathrm{~cm} \phi, 15 \mathrm{~cm}$ column, $3 \% \mathrm{MeOH}$ in $\mathrm{CO}_{2}$, flow rate: $1.2 \mathrm{~mL} / \mathrm{min}$; $t \mathrm{R}: 3.22 \mathrm{~min}$ (minor enantiomer), $5.11 \mathrm{~min}$ (major enantiomer); $e e$ (major enantiomer) $=89 \%$.

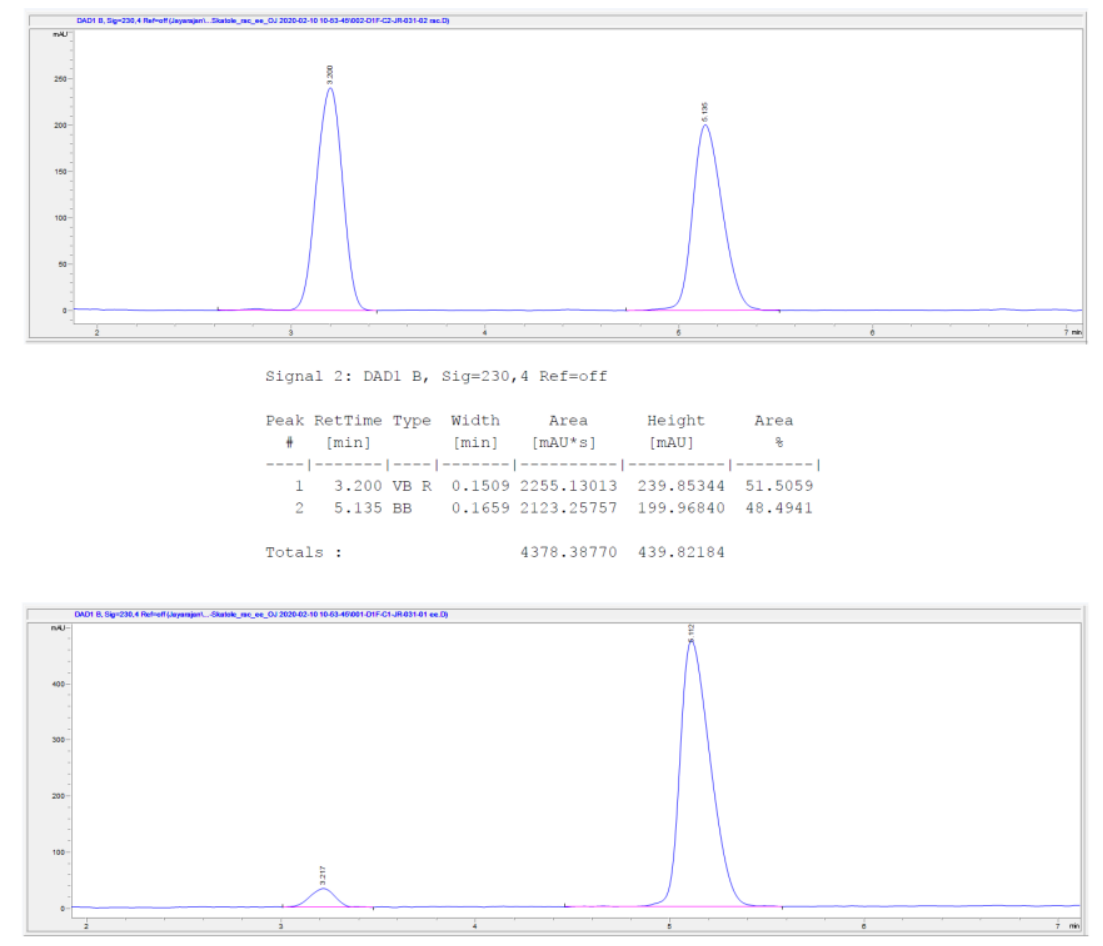

Signal 2: DAD1 B, Sig=230, 4 Ref $=$ off

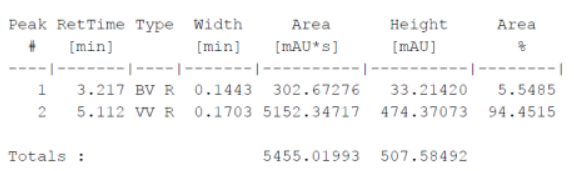


(S)-1-((R,E)-4,4,4-trifluoro-1-phenylbut-2-en-1-yl)-1,2,3,4-tetrahydroisoquinoline (7l): This<smiles>FC(F)(F)/C=C/[C@H](c1ccccc1)C1NCCc2ccccc21</smiles>
compound was obtained according to the above general procedure E. Product 71 was isolated in $54 \%$ yield $(17.1 \mathrm{mg}, 54 \mu \mathrm{mol})$ as colorless viscous oil by silica gel chromatography using petroleum ether/ethyl acetate 20:1 as eluent.

${ }^{1}$ H NMR (400 MHz, $\left.\mathbf{C D C l}_{3}\right) \boldsymbol{\delta} 7.43-7.34(\mathrm{~m}, 2 \mathrm{H}), 7.30(\mathrm{td}, J=6.5,1.7$ $\mathrm{Hz}, 3 \mathrm{H}), 7.19-7.05(\mathrm{~m}, 3 \mathrm{H}), 6.98(\mathrm{~d}, J=7.8 \mathrm{~Hz}, 1 \mathrm{H}), 6.75$ (ddq, $J=16.0$, 8.2, $2.1 \mathrm{~Hz}, 1 \mathrm{H}), 5.53(\mathrm{dqd}, J=16.0,6.4,1.2 \mathrm{~Hz}, 1 \mathrm{H}), 4.39(\mathrm{~d}, J=4.5 \mathrm{~Hz}, 1 \mathrm{H}), 4.14-3.84(\mathrm{~m}$, 1H), $3.31-3.07(\mathrm{~m}, 1 \mathrm{H}), 3.07-2.79(\mathrm{~m}, 2 \mathrm{H}), 2.78-2.58(\mathrm{~m}, 1 \mathrm{H}), 1.67(\mathrm{~s}, 1 \mathrm{H}) ;{ }^{13} \mathbf{C}$ NMR (101 $\left.\mathbf{M H z}, \mathbf{C D C l}_{3}\right) \boldsymbol{\delta} 140.70,139.61\left(\mathrm{q},{ }^{3} J_{C-F}=6.7 \mathrm{~Hz}\right), 136.47,136.19,129.47,128.98,128.71$, 127.32, 126.51, 126.44, 125.77, $122.99\left(\mathrm{q},{ }^{1} J_{C-F}=269.5 \mathrm{~Hz}\right), 120.87\left(\mathrm{q},{ }^{2} J_{C-F}=33.3 \mathrm{~Hz}\right), 60.57$, 52.90, 41.79, 30.17; ${ }^{19} \mathbf{F}$ NMR (377 MHz, CDCl $) \boldsymbol{\delta}-64.00(\mathrm{dt}, J=6.5,2.0 \mathrm{~Hz}$ ). HRMS (pos. ESI) $\mathbf{m} / \mathbf{z}$ : calcd. for $\mathrm{C}_{19} \mathrm{H}_{19} \mathrm{~F}_{3} \mathrm{~N}[\mathrm{M}+\mathrm{H}]^{+}$318.1464. Found 318.1459. $[\alpha]_{D}^{26}=-42.4(c 0.375$, $\left.\mathrm{CHCl}_{3}\right)$.

Determination of $\boldsymbol{e} e$ by Chiral HPLC: Diacel CHIRALCEL OJ-H, $25{ }^{\circ} \mathrm{C}, 0.46 \mathrm{~cm} \phi, 0.25 \mathrm{~cm}$ column, $10 \%{ }^{\mathrm{i}} \mathrm{PrOH}$ in Hexane, flow rate: $1.0 \mathrm{~mL} / \mathrm{min}$; $t \mathrm{R}: 5.81 \mathrm{~min}$ (minor enantiomer), $7.07 \mathrm{~min}$ (major enantiomer); ee (major enantiomer) $=93 \%$.
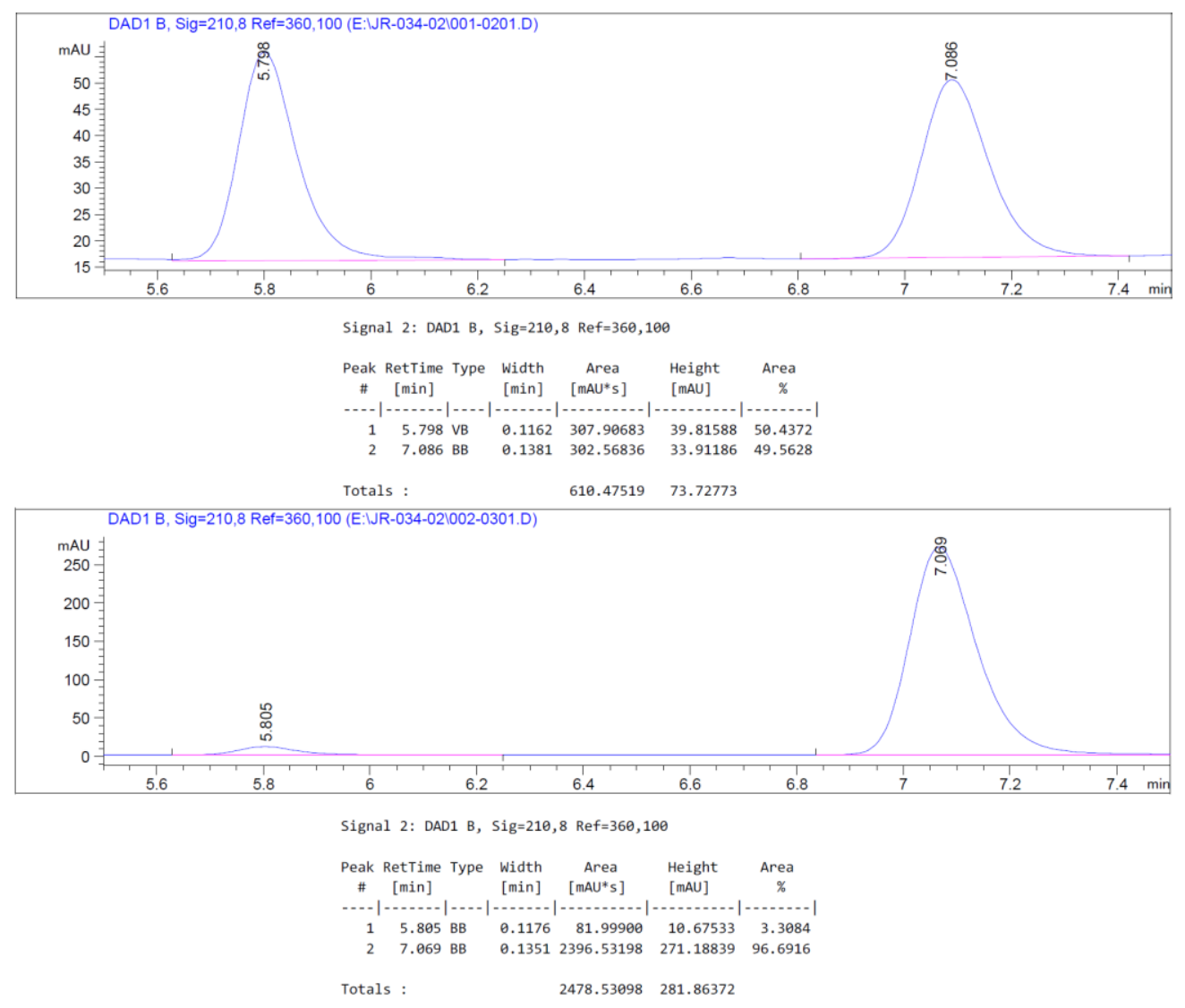
Ethyl (2S,3S)-2-(2-benzoylhyd razineyl)-3-((E)-3,3,3-trifluoroprop-1-en-1-yl)nonanoate<smiles>CCCCCC[C@H](/C=C/C(F)(F)F)[C@H](NNC(=O)c1ccccc1)C(=O)OCC</smiles>

(7m): This compound was obtained according to the above general procedure E except 0.9 equiv $(0.09 \mathrm{mmol})$ of $\mathbf{6 i}$ was used. Product $\mathbf{7 m}$ was isolated in $72 \%$ yield $(29.7 \mathrm{mg}, 72 \mu \mathrm{mol})$ as a colorless oil by silica gel chromatography starting from a dry loading and using pentane/ethyl acetate 40:1 to 10:1 as eluent.

${ }^{1}$ H NMR (400 MHz, CDCl $) \boldsymbol{\delta} 8.00(\mathrm{~s}, 1 \mathrm{H}), 7.79-7.64(\mathrm{~m}, 2 \mathrm{H})$, $7.57-7.48(\mathrm{~m}, 1 \mathrm{H}), 7.43(\mathrm{dd}, J=8.3,6.8 \mathrm{~Hz}, 2 \mathrm{H}), 6.48-6.18(\mathrm{~m}$, $1 \mathrm{H}), 5.74(\mathrm{dq}, J=15.8,6.2 \mathrm{~Hz}, 1 \mathrm{H}), 4.59(\mathrm{~s}, 1 \mathrm{H}), 4.20(\mathrm{qd}, J=7.1$, $2.7 \mathrm{~Hz}, 2 \mathrm{H}), 3.76(\mathrm{~d}, J=5.9 \mathrm{~Hz}, 1 \mathrm{H}), 2.59(\mathrm{dt}, J=9.9,4.9 \mathrm{~Hz}, 1 \mathrm{H}), 1.70(\mathrm{tt}, J=9.1,4.1 \mathrm{~Hz}, 1 \mathrm{H})$, $1.48(\mathrm{dtd}, J=13.3,9.1,3.0 \mathrm{~Hz}, 1 \mathrm{H}), 1.26(\mathrm{t}, J=7.1 \mathrm{~Hz}, 10 \mathrm{H}), 0.94-0.79(\mathrm{~m}, 3 \mathrm{H}) ;{ }^{13} \mathbf{C}$ NMR

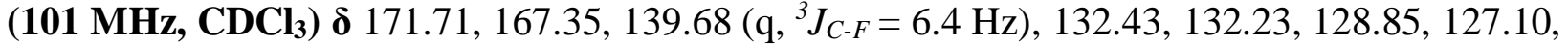
$122.75\left(\mathrm{q},{ }^{1} J_{C-F}=267.3 \mathrm{~Hz}\right), 120.92\left(\mathrm{q},{ }^{2} J_{C-F}=33.6 \mathrm{~Hz}\right), 66.44,61.50,43.66,31.71,30.43,29.14$,

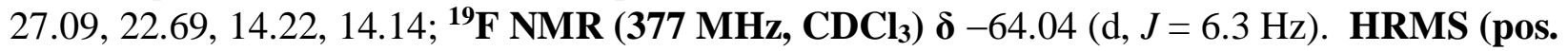
ESI) $\mathbf{m} / \mathbf{z}$ : calcd for $\mathrm{C}_{21} \mathrm{H}_{29} \mathrm{~F}_{3} \mathrm{~N}_{2} \mathrm{NaO}_{3}[\mathrm{M}+\mathrm{Na}]^{+}$437.2022. Found 437.2031. $[\boldsymbol{\alpha}]_{\boldsymbol{D}}^{\mathbf{2 5}}=-14.9(c 0.65$, $\left.\mathrm{CHCl}_{3}\right)$.

Determination of $\boldsymbol{e} e$ by Chiral SFC: Diacel CHIRALPAK IB N-3, $25^{\circ} \mathrm{C}, 0.3 \mathrm{~cm} \phi, 15 \mathrm{~cm}$ column, $1 \% \mathrm{MeOH}$ in $\mathrm{CO}_{2}$, flow rate: $2.0 \mathrm{~mL} / \mathrm{min}$; $t \mathrm{R}: 11.67 \mathrm{~min}$ (minor enantiomer), 13.19 min (major enantiomer); ee (major enantiomer) $=98 \%$.
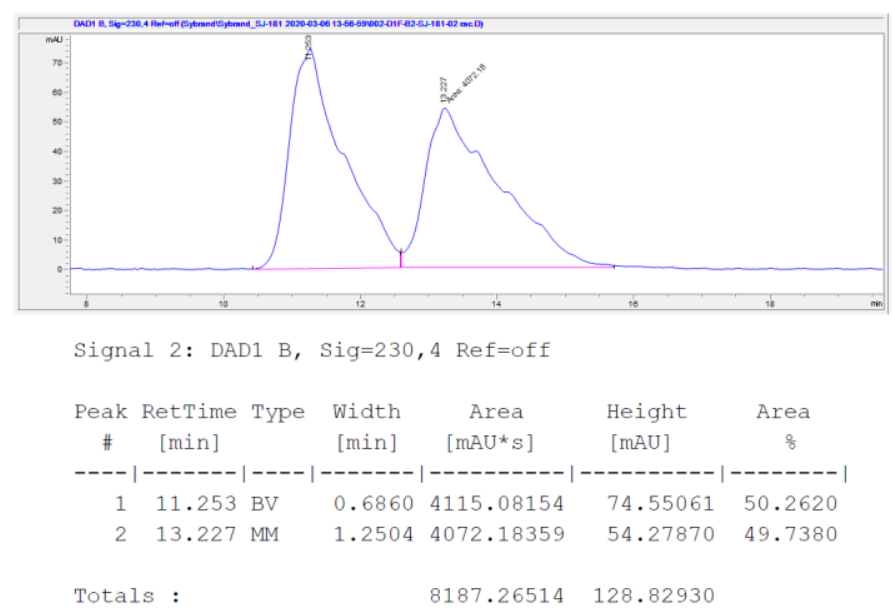

Totals : $\quad 8187.26514 \quad 128.82930$
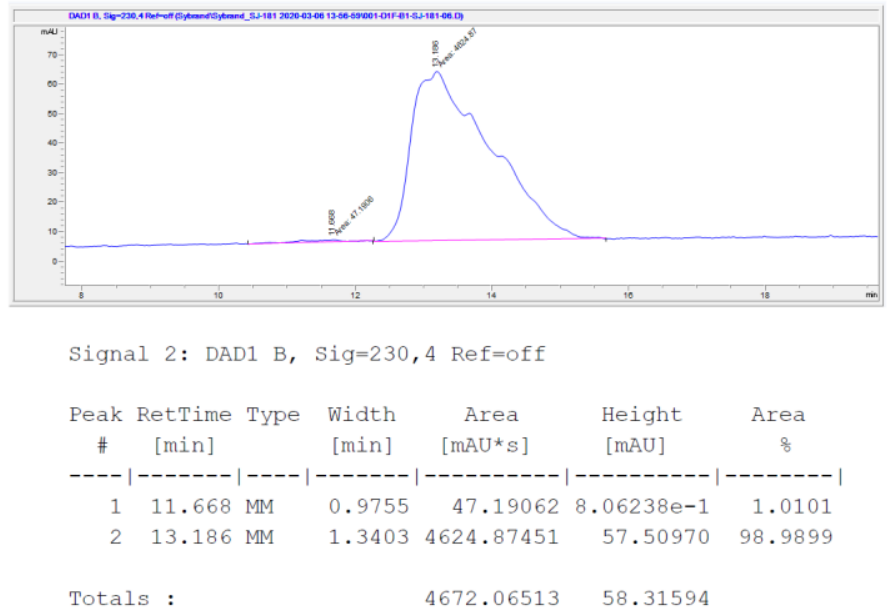
(S,E)-1,1,1-Trifluorodec-3-en-2-ol (8a): This compound was obtained according to the general<smiles>CCCCCC/C=C/C(O)C(F)(F)F</smiles>
procedure D. Product 8a was isolated in $78 \%$ yield $(16.4 \mathrm{mg}, 78$ $\mu \mathrm{mol})$ as a volatile light yellow oil by silica gel chromatography using pentane/DCM eluent gradient 5:1 to $1: 1$.

${ }^{1} \mathbf{H}$ NMR (400 MHz, CDCl $) \boldsymbol{\delta} 5.98(\mathrm{dt}, J=15.1,7.3 \mathrm{~Hz}, 1 \mathrm{H}), 5.51(\mathrm{dd}, J=15.5,7.0 \mathrm{~Hz}, 1 \mathrm{H})$, $4.39(\mathrm{p}, J=6.7 \mathrm{~Hz}, 1 \mathrm{H}), 2.11(\mathrm{q}, J=6.7 \mathrm{~Hz}, 2 \mathrm{H}), 1.40(\mathrm{p}, J=6.2 \mathrm{~Hz}, 2 \mathrm{H}), 1.35-1.22(\mathrm{~m}, 6 \mathrm{H})$, $0.90-0.87(\mathrm{t}, J=7.05 \mathrm{~Hz}, 3 \mathrm{H}) .{ }^{13} \mathbf{C} \mathbf{N M R}\left(\mathbf{1 0 1} \mathbf{~ M H z}, \mathbf{C D C l}_{3}\right) \boldsymbol{\delta} 139.39,124.51\left(\mathrm{q},{ }^{1} J_{C-F}=281.6\right.$ $\mathrm{Hz}), 122.06\left(\mathrm{q},{ }^{3} J_{C-F}=1.9 \mathrm{~Hz}\right), 71.82\left(\mathrm{q},{ }^{2} J_{C-F}=32.0 \mathrm{~Hz}\right), 32.42,31.76,28.86,28.69,22.72,14.19$. ${ }^{19} \mathbf{F}$ NMR $\left(377 \mathrm{MHz}, \mathbf{C D C l}_{3}\right) \delta \boldsymbol{\delta}-75.5(\mathrm{~d}, J=6.4 \mathrm{~Hz})$. After repeated attempts we were unable to obtain HRMS data for compound 8a, probably due to its resistance to ionization. GC-MS m/z: 70 (100), 69 (97), 81 (36), 67 (26), 83 (24), 71 (20), 84 (19). $[\alpha]_{D}^{27}=-4.286$ (c 0.21, $\mathrm{CHCl}_{3}$ ).

Determination of $e e$ by Chiral GC: Column Chiraldex $\beta-6 \mathrm{TBDM}, 50^{\circ} \mathrm{C}$ to $110^{\circ} \mathrm{C}$ at $1{ }^{\circ} \mathrm{C} / \mathrm{min}$, $\mathrm{tR}=48.06 \mathrm{~min}$ (major enantiomer); $e e$ (major enantiomer) $=99 \%$.

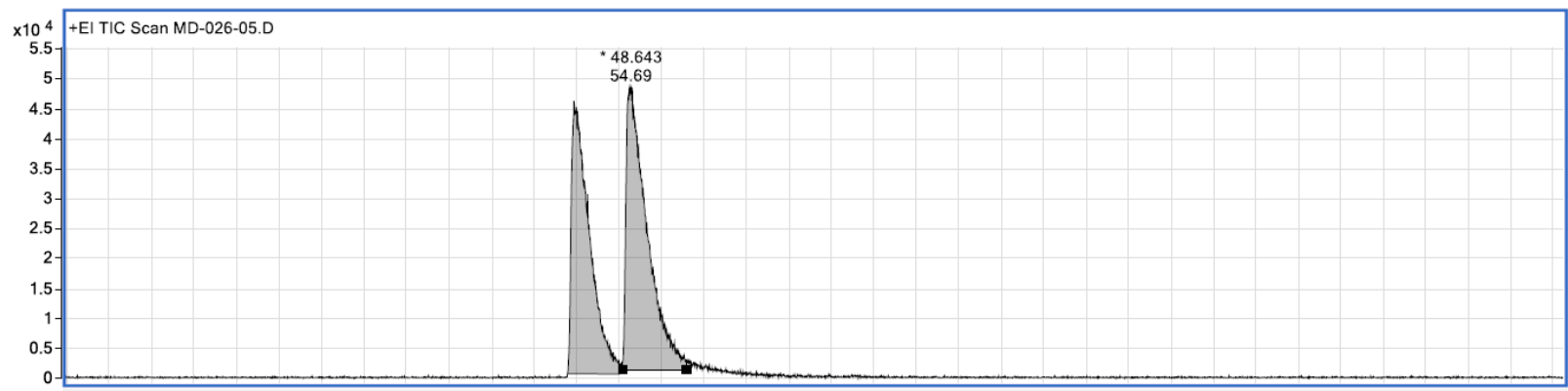

\begin{tabular}{|c|c|c|c|c|c|c|c|}
\hline Peak & $\mapsto$ & Area Sum \% & $\mathrm{RT} \mapsto$ & Area & Width $\mapsto$ & FWHM & Height \\
\hline & 1 & 45.31 & 47.97 & 670022.9 & 0.613 & 0.227 & 45587.6 \\
\hline & 2 & 54.69 & 48.643 & 808648.8 & 0.757 & 0.253 & 47288.5 \\
\hline
\end{tabular}

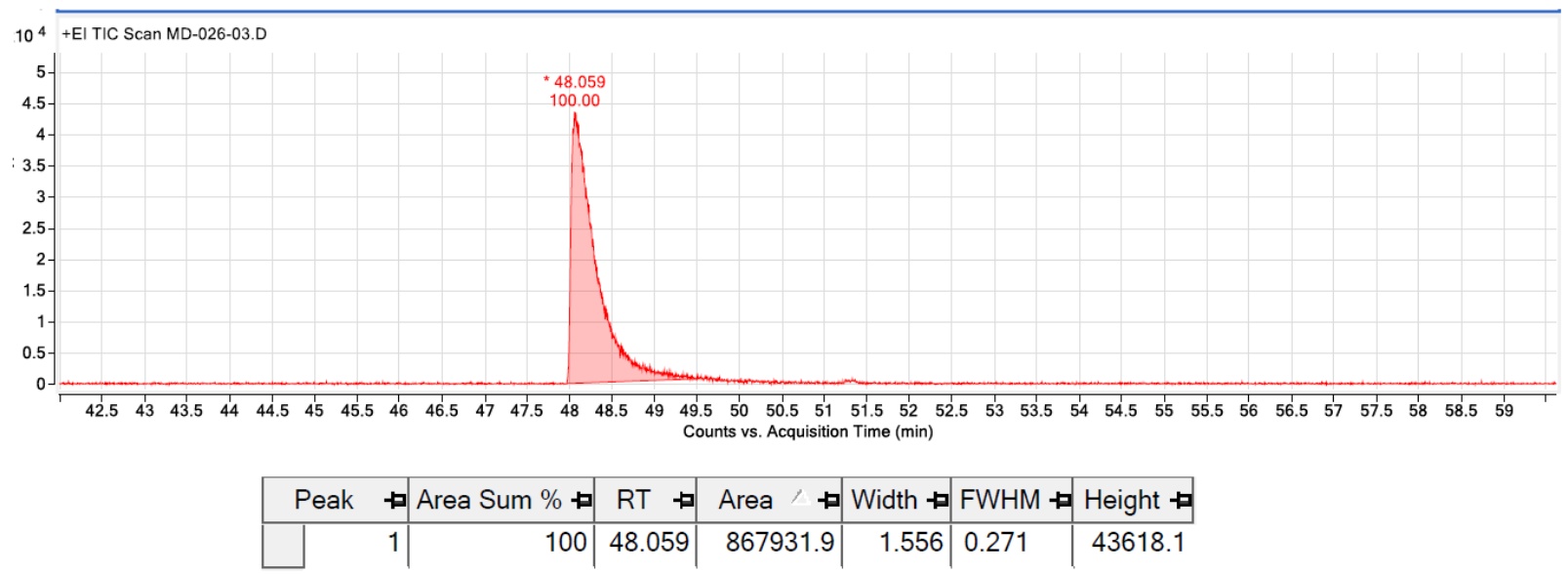


(S,E)-1,1,1-trifluoro-5-phenylpent-3-en-2-ol (8b): This compound was obtained according to<smiles>OC(C=CCc1ccccc1)C(F)(F)F</smiles>
the above general procedure. Product $\mathbf{8 b}$ was isolated in 50\% yield (10.9 $\mathrm{mg}, 50 \mu \mathrm{mol})$ as colorless oil by silica gel chromatography using pentane/DCM eluent gradient from 2:1 to 1:1.

${ }^{1} \mathbf{H}$ NMR (400 MHz, $\left.\mathbf{C D C l}_{3}\right) \boldsymbol{\delta} 7.36-7.29(\mathrm{~m}, 2 \mathrm{H}), 7.26-7.21(\mathrm{~m}, 1 \mathrm{H})$, $7.20-7.15(\mathrm{~m}, 2 \mathrm{H}), 6.15(\mathrm{dtd}, J=15.1,6.8,1.2 \mathrm{~Hz}, 1 \mathrm{H}), 5.59(\mathrm{dd}, J=15.5,6.7 \mathrm{~Hz}, 1 \mathrm{H}), 4.45$ (p, $J=6.5 \mathrm{~Hz}, 1 \mathrm{H}), 3.46(\mathrm{~d}, J=6.8 \mathrm{~Hz}, 2 \mathrm{H}), 2.15(\mathrm{~s}, 1 \mathrm{H}) .{ }^{13} \mathbf{C} \mathbf{~ N M R}\left(\mathbf{1 0 1} \mathbf{M H z}, \mathbf{C D C l}_{3}\right) \boldsymbol{\delta} 138.90$, $137.35,128.75,128.72,126.61,124.41\left(\mathrm{q},{ }^{1} J_{C-F}=281.8 \mathrm{~Hz}\right), 123.47\left(\mathrm{q},{ }^{3} J_{C-F}=2.0 \mathrm{~Hz}\right), 71.53(\mathrm{q}$, $J=32.2 \mathrm{~Hz}), 38.75 .{ }^{19} \mathbf{F}$ NMR $\left(377 \mathbf{M H z}, \mathbf{C D C l}_{3}\right) \boldsymbol{\delta}-79.37$ (d, $\left.J=6.8 \mathrm{~Hz}\right) . \mathrm{GC}-\mathrm{MS} \mathrm{m} / \mathrm{z}: 216(\mathrm{M}+$ 14), 117 (100), 129 (51), 91 (42), 115 (37), 198 (13). $[\boldsymbol{\alpha}]_{D}^{22}=-12.4\left(c 0.25, \mathrm{CHCl}_{3}\right)$.

Determination of $e \boldsymbol{e}$ by Chiral GC: Column Chiraldex $\beta-6 \mathrm{TBDM}, 50^{\circ} \mathrm{C}$ to $110^{\circ} \mathrm{C}$ at $1{ }^{\circ} \mathrm{C} / \mathrm{min}$, $\mathrm{tR}=70.05 \mathrm{~min}$ (major enantiomer), $\mathrm{tR}=70.77 \mathrm{~min}$ (minor enantiomer); $e e$ (major enantiomer) $=$ $96 \%$.
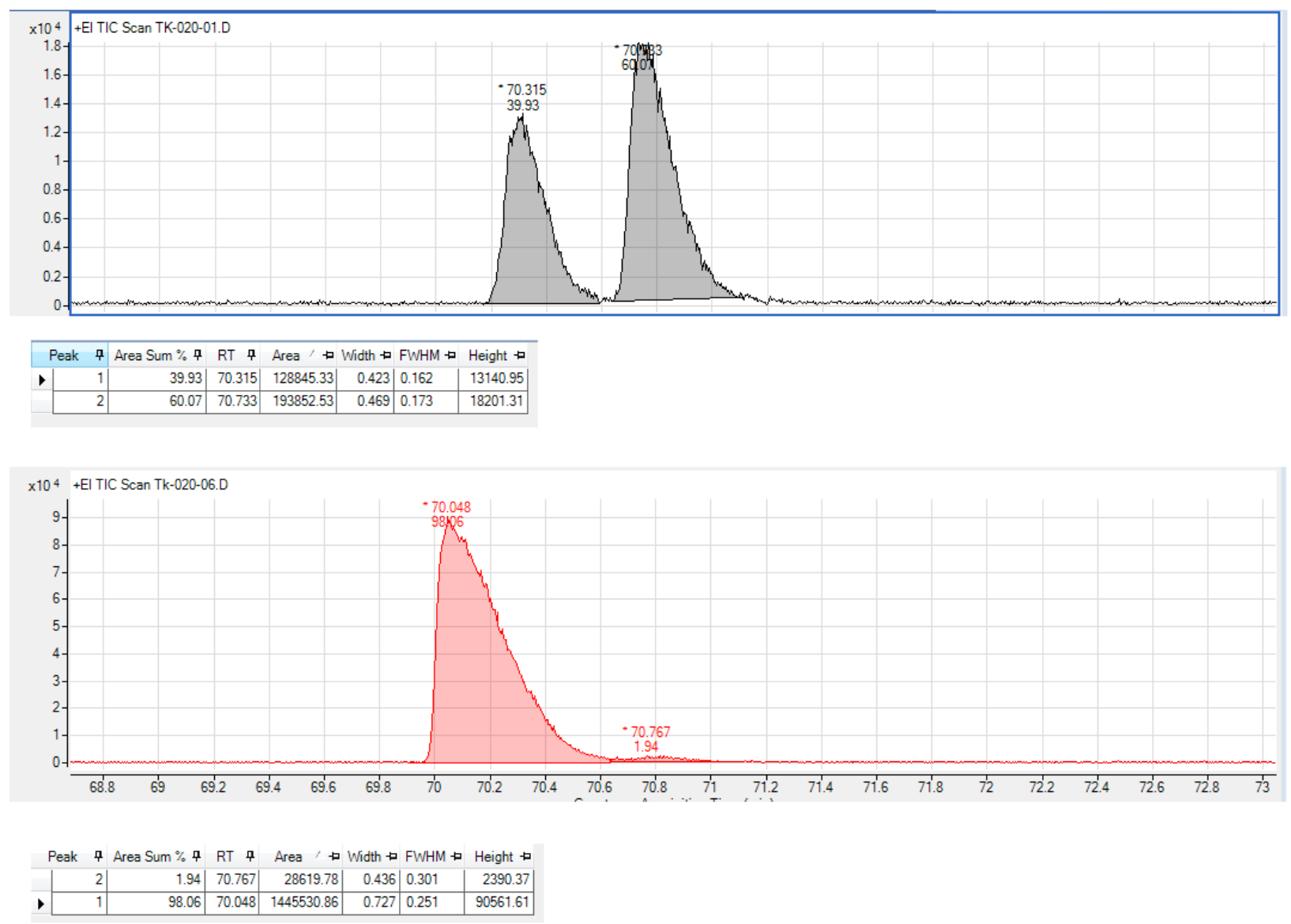
(S,E)-1,1,1-trifluoro-4-phenylbut-3-en-2-ol (8c): This compound was obtained according to the<smiles>OC(/C=C/c1ccccc1)C(F)(F)F</smiles>
general procedure $\mathrm{D}$, except that before oxidation, pinacol $(0.15 \mathrm{mmol})$ was added to the reaction mixture and was stirred for 1 hour at $40{ }^{\circ} \mathrm{C}$. Product $8 \mathbf{c}$ was isolated in $64 \%$ yield $(13.1 \mathrm{mg}, 64 \mu \mathrm{mol})$ as white solid by silica gel chromatography using pentane/DCM, 2:1 as eluent. $\mathrm{Mp}=55.7^{\circ} \mathrm{C}$.

${ }^{1} \mathbf{H}$ NMR (400 MHz, $\left.\mathbf{C D C l}_{3}\right) \boldsymbol{\delta} 7.47-7.40(\mathrm{~m}, 2 \mathrm{H}), 7.40-7.28(\mathrm{~m}, 3 \mathrm{H}), 6.87(\mathrm{dd}, J=15.9,1.2$ $\mathrm{Hz}, 1 \mathrm{H}), 6.21$ (dd, $J=15.9,6.5 \mathrm{~Hz}, 1 \mathrm{H}), 4.64$ (hd, $J=6.5,1.4 \mathrm{~Hz}, 1 \mathrm{H}), 2.31(\mathrm{~d}, J=5.9 \mathrm{~Hz}, 1 \mathrm{H})$.

${ }^{13}$ C NMR (101 MHz, CDCl $)$ ) $\boldsymbol{\delta} 136.46,135.47,128.90,128.88,127.03,124.40\left(\mathrm{q},{ }^{1} J_{C-F}=281.9\right.$ $\mathrm{Hz}), 120.76\left(\mathrm{q},{ }^{3} J_{C-F}=2.0 \mathrm{~Hz}\right), 71.78\left(\mathrm{q},{ }^{2} J_{C-F}=32.4 \mathrm{~Hz}\right) .{ }^{\mathbf{1}} \mathbf{F} \mathbf{~ N M R}\left(\mathbf{3 7 7} \mathbf{~ M H z}, \mathbf{C D C l}_{\mathbf{3}}\right) \boldsymbol{\delta}-79.08$ (d, $J=6.7 \mathrm{~Hz}$ ). GC-MS m/z: 202 (M+, 42), 133 (100), 115 (38), 77 (30), 91 (23), 103 (22), 105 (21). $[\boldsymbol{\alpha}]_{\boldsymbol{D}}^{24}=+6.4\left(c 0.25, \mathrm{CHCl}_{3}\right)$.

Determination of $e \boldsymbol{e}$ by Chiral SFC: Diacel CHIRALCEL OJ-H, $25^{\circ} \mathrm{C}, 0.3 \mathrm{~cm} \phi, 15 \mathrm{~cm}$ column, $20 \% \mathrm{MeOH}$ in $\mathrm{CO}_{2}$, flow rate: $1.2 \mathrm{~mL} / \mathrm{min}$; $\mathrm{tR}: 9.65 \mathrm{~min}$ (major enantiomer), 11.0 min (minor enantiomer); ee (major enantiomer) $=94 \%$.

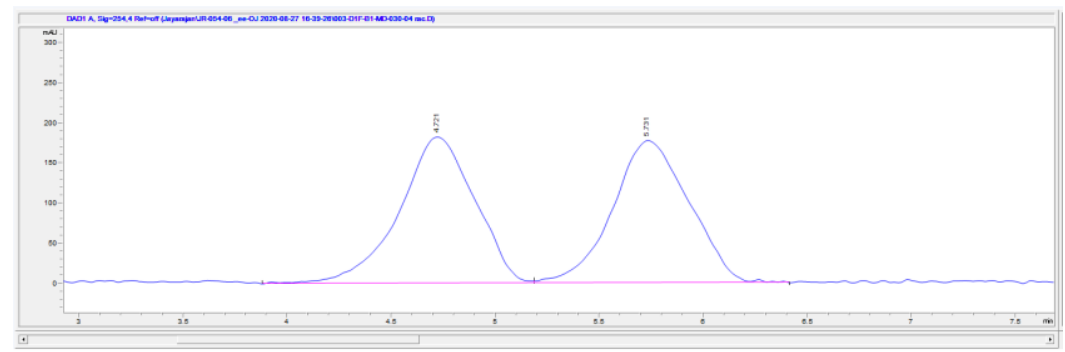

Signal 1: DAD1 A, Sig=254, 4 Ref $=0 f f$

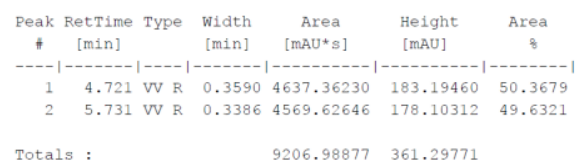

Totals : $\quad 9206.98877 \quad 361.29771$

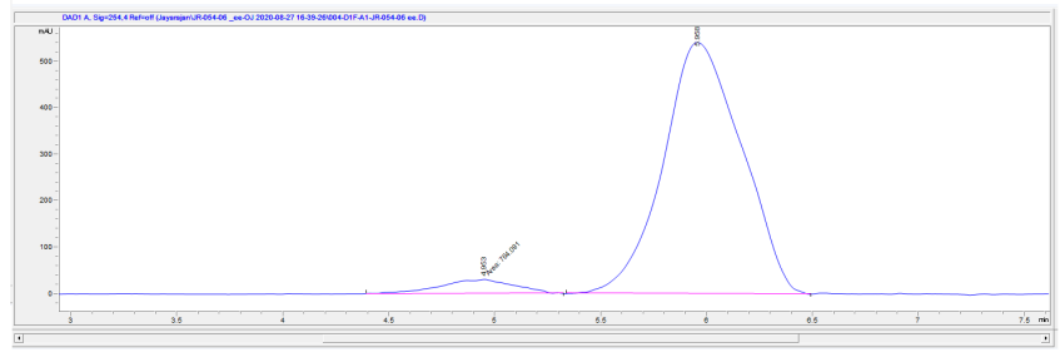

Signal 1: DAD1 A, Sig=254,4 Ref=off

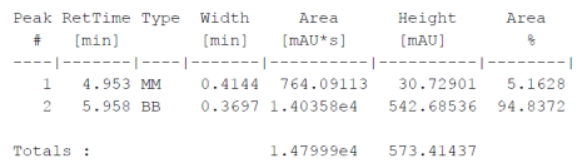


((S,E)-3-(4-fluorophenyl)-1-((3aS,4S,6S,7aR)-3a,5,5-trimethylhexahydro-4,6-methano

benzo[d][1,3,2]dioxaborol-2-yl)allyl)trimethylsilane (9): This compound was obtained<smiles>C[C@H](/C=C/c1ccc(F)cc1)B1O[C@H]2CC[C@@H](CC2(C)C)C1(C)C</smiles>
according to the above general procedure B. Product 9 was isolated in $51 \%$ yield $(19.5 \mathrm{mg}, 51 \mu \mathrm{mol})$ as white solid by silica gel chromatography using petroleum ether/ethyl acetate 40:1 as eluent. M.pt.: $98.3^{\circ} \mathrm{C}$.

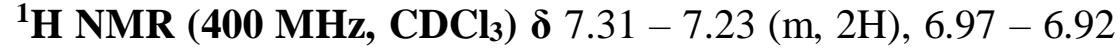
$(\mathrm{m}, 2 \mathrm{H}), 6.26(\mathrm{dd}, J=15.7,10.6 \mathrm{~Hz}, 1 \mathrm{H}), 6.14(\mathrm{~d}, J=15.7 \mathrm{~Hz}$, $1 \mathrm{H}), 4.28(\mathrm{dd}, J=8.8,2.1 \mathrm{~Hz}, 1 \mathrm{H}), 2.51-2.30(\mathrm{~m}, 1 \mathrm{H}), 2.29-2.15(\mathrm{~m}, 1 \mathrm{H}), 2.07(\mathrm{dd}, J=6.0$, $5.0 \mathrm{~Hz}, 1 \mathrm{H}), 1.91(\mathrm{tt}, J=5.7,2.9 \mathrm{~Hz}, 1 \mathrm{H}), 1.85(\mathrm{ddd}, J=14.5,3.3,2.1 \mathrm{~Hz}, 1 \mathrm{H}), 1.71(\mathrm{~d}, J=10.5$ $\mathrm{Hz}, 1 \mathrm{H}), 1.39(\mathrm{~s}, 3 \mathrm{H}), 1.29(\mathrm{~s}, 3 \mathrm{H}), 1.16(\mathrm{~d}, J=10.8 \mathrm{~Hz}, 1 \mathrm{H}), 0.85(\mathrm{~s}, 3 \mathrm{H}), 0.10(\mathrm{~s}, 9 \mathrm{H}) ;{ }^{13} \mathbf{C ~ N M R}$ (101 MHz, CDCl $)$ ) $162.75,160.32,135.07,135.04,128.89,128.86,126.99,126.91,126.13$, $115.35,115.14,85.71,77.75,51.42,39.71,38.30,35.84,29.00,27.24,26.77,24.18,-1.37 ;{ }^{19} \mathbf{F}$ NMR (377 MHz, CDCl 3 ) $\delta$-117.18; ${ }^{11} \mathrm{~B}_{\mathrm{NMR}}\left(\mathbf{1 2 8} \mathrm{MHz}_{\mathrm{CDCl}}\right.$ ) $\delta$ 33.16. HRMS (pos. ESI) $\mathbf{m} / \mathbf{z}$ : calcd for $\mathrm{C}_{22} \mathrm{H}_{32} \mathrm{BFNaO}_{2} \mathrm{Si}[\mathrm{M}+\mathrm{Na}]^{+}$409.2145, Found 409.2149. $[\boldsymbol{\alpha}]_{\boldsymbol{D}}^{\mathbf{2 6}}=-16.00$ (c 0.45, $\left.\mathrm{CHCl}_{3}\right)$.

Determination of $\boldsymbol{e} e$ by Chiral SFC: Diacel CHIRALPAK OZ-H N-3, $40{ }^{\circ} \mathrm{C}, 0.46 \mathrm{~cm} \phi, 25 \mathrm{~cm}$ column, $3 \% \mathrm{MeOH}$ in $\mathrm{CO}_{2}$, flow rate: $2.0 \mathrm{~mL} / \mathrm{min}$; $t \mathrm{R}: 4.16 \mathrm{~min}$ (major enantiomer), $4.75 \mathrm{~min}$ (minor enantiomer); ee (major enantiomer) $=98 \%$.
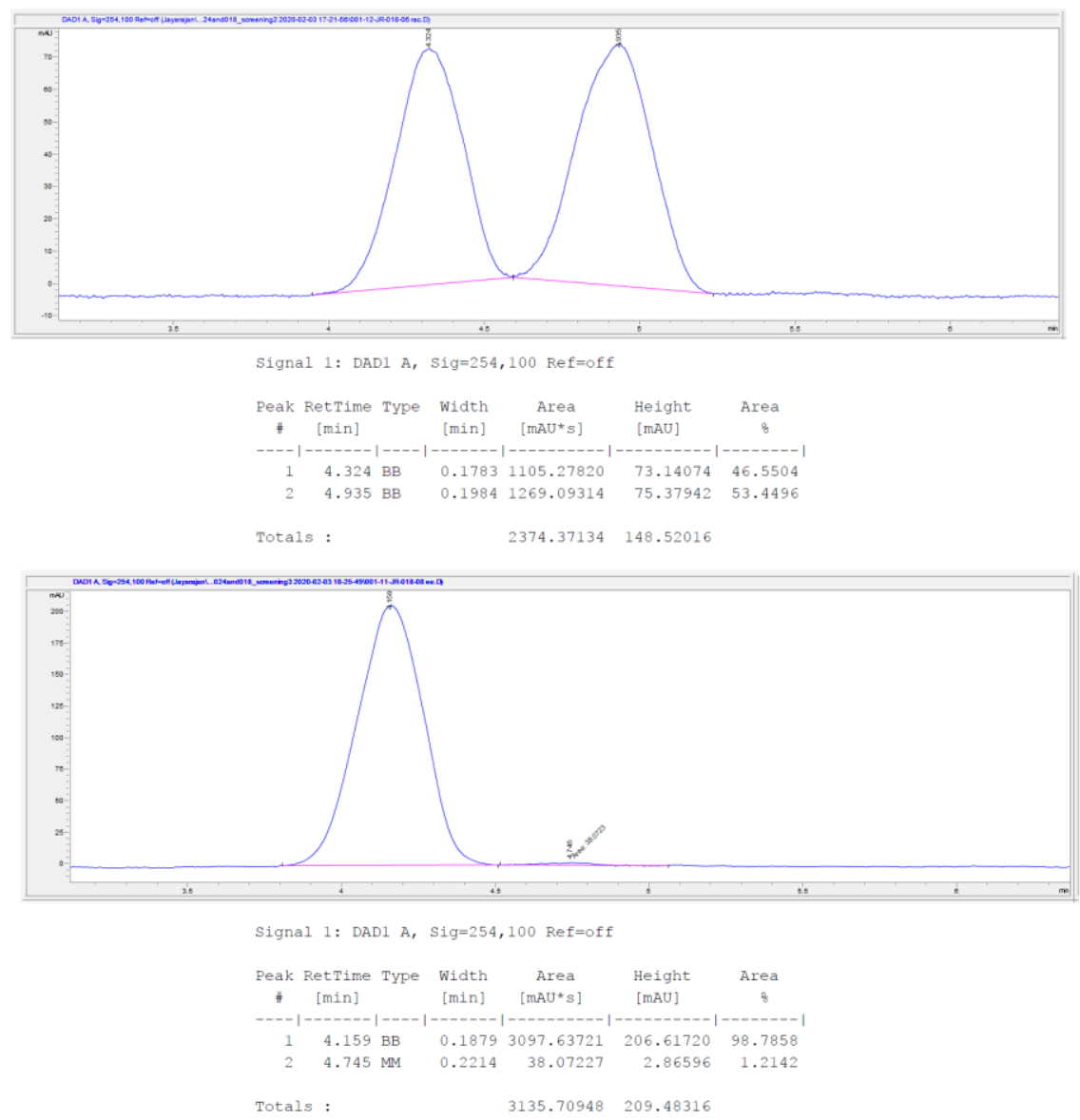
(1S,2R,E)-1-(4-bromophenyl)-2-(4-fluorophenyl)-4-(trimethylsilyl)but-3-en-1-ol (10): This<smiles>CCC=CC(O)c1ccc(Br)cc1</smiles>
compound was obtained according to the above general procedure B in step 1 using iodo-BINOL $4(0.03 \mathrm{mmoL})$ and general procedure C in step 2. Product 10 was isolated in $52 \%$ yield $(20.6 \mathrm{mg}, 52 \mu \mathrm{mol})$ as yellowish oil by silica gel chromatography using petroleum ether/ethyl acetate $40: 1$ as eluent.

${ }^{1} \mathbf{H}$ NMR (400 MHz, $\left.\mathbf{C D C l}_{3}\right) \boldsymbol{\delta} 7.34-7.29(\mathrm{~m}, 2 \mathrm{H}), 7.01-6.84(\mathrm{~m}$, $6 \mathrm{H}), 6.31(\mathrm{dd}, J=18.5,8.3 \mathrm{~Hz}, 1 \mathrm{H}), 5.88(\mathrm{dd}, J=18.5,1.0 \mathrm{~Hz}, 1 \mathrm{H})$, $4.75(\mathrm{~d}, J=7.9 \mathrm{~Hz}, 1 \mathrm{H}), 3.46(\mathrm{td}, J=8.2,1.1 \mathrm{~Hz}, 1 \mathrm{H}), 2.31(\mathrm{~s}, 1 \mathrm{H}), 0.08(\mathrm{~s}, 9 \mathrm{H}) ;{ }^{\mathbf{1 3}} \mathbf{C}$ NMR $(\mathbf{1 0 1}$ $\left.\mathbf{M H z}_{\mathbf{1}} \mathbf{C D C l}_{3}\right) \boldsymbol{\delta} 162.95,160.51,144.56,140.78,136.01,135.92,135.89,132.24,131.43,131.15$, 129.96, 129.88, 128.53, 121.43, 115.54, 115.33, 76.82, 76.70, 61.33, -1.14; ${ }^{\mathbf{1 9}}$ F NMR (377 MHz, $\left.\mathbf{C D C l}_{3}\right) \boldsymbol{\delta}-115.88$. HRMS (pos. ESI) $\mathbf{m} / \mathbf{z}$ : calcd for. $\mathrm{C}_{19} \mathrm{H}_{22} \mathrm{BrFNaOSi}[\mathrm{M}+\mathrm{Na}]^{+}$415.0500, Found. 415,0505. $[\boldsymbol{\alpha}]_{\boldsymbol{D}}^{26}=-3.33\left(c 0.30, \mathrm{CHCl}_{3}\right)$.

Determination of $e \boldsymbol{e}$ by Chiral SFC: Diacel CHIRALPAK IA-3, $25^{\circ} \mathrm{C}, 0.3 \mathrm{~cm} \phi, 15 \mathrm{~cm}$ column, $1 \% \mathrm{MeOH}$ in $\mathrm{CO}_{2}$, flow rate: $0.8 \mathrm{~mL} / \mathrm{min}$; $t \mathrm{R}: 9.27$ min (minor enantiomer), 10.62 min (major enantiomer); $e e($ major enantiomer $)=91 \%$.

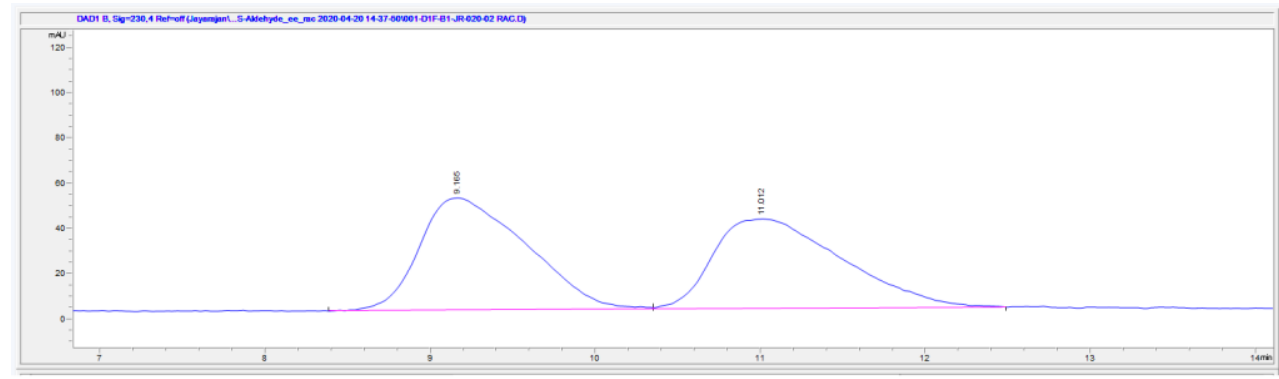

Signal 2: DAD1 B, Sig=230, 4 Ref $=$ off
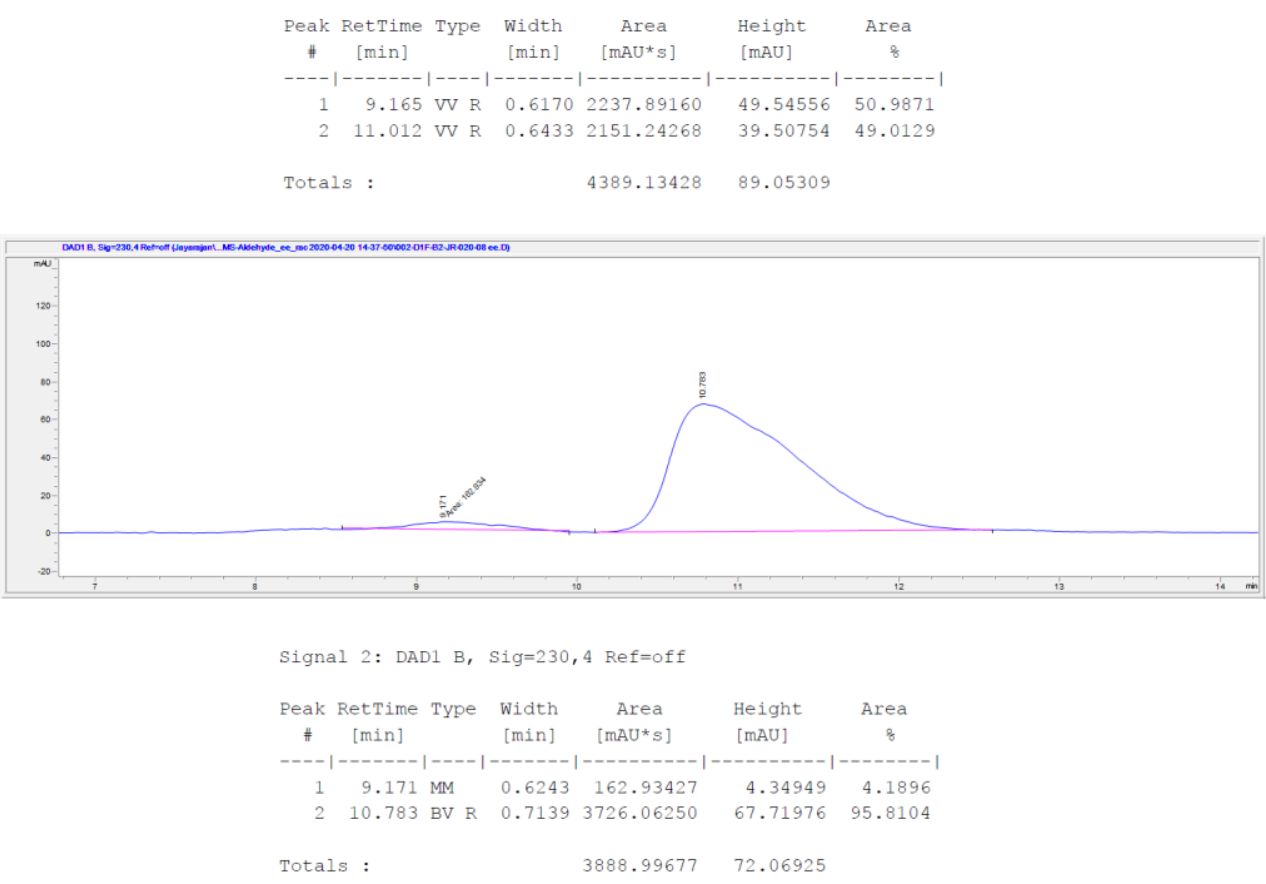


\subsection{NMR spectra of purified allylboronic acids}

Preparation of the NMR sample for $(S, E)-(1,1,1$-trifluorodec-3-en-2-yl)boronic acid (1a):<smiles>CCCCCC/C=C/C(B(O)O)C(F)(F)F</smiles>
Allyl-Bdan $\mathbf{5 a}(0.1 \mathrm{mmol})$ was taken in a screw capped reaction tube under argon. $600 \mu \mathrm{L}$ degassed DME was added followed by $500 \mu \mathrm{L} 3 \mathrm{~N} \mathrm{H}_{2} \mathrm{SO}_{4}$. The mixture was stirred overnight at room temperature. Then, $0.5 \mathrm{M} \mathrm{HCl}$ was added as well as $\mathrm{Et}_{2} \mathrm{O}$ and shaken vigorously. The $\mathrm{Et}_{2} \mathrm{O}$ layer was passed through a phase separator and evaporated. $\mathrm{CDCl}_{3}$ was added and NMR data was recorded. The conversion of allyl-Bdan 5a into free boronic acid 1a was found to be $96 \%$ based on ${ }^{19} \mathrm{~F}$ NMR analysis by comparison to the signal at $-62.42 \mathrm{ppm}$ that belongs to a $4 \%$ trace of allyl-Bdan $\mathbf{5 a}$ (See ${ }^{1} \mathrm{H}-\mathrm{NMR}$ below). ${ }^{1} \mathbf{H}$ NMR $\left(400 \mathrm{MHz}, \mathbf{C D C l}_{3}\right) \boldsymbol{\delta}$ $5.68(\mathrm{dt}, J=14.2,6.7 \mathrm{~Hz}, 1 \mathrm{H}), 5.44(\mathrm{ddt}, J=15.4,9.1,1.5 \mathrm{~Hz}, 1 \mathrm{H}), 2.65(\mathrm{p}, J=11.6 \mathrm{~Hz}, 1 \mathrm{H})$, $2.12-2.01(\mathrm{~m}, 2 \mathrm{H}), 1.43-1.34(\mathrm{~m}, 2 \mathrm{H}), 1.33-1.26(\mathrm{~m}, 6 \mathrm{H}), 0.92-0.81(\mathrm{~m}, 3 \mathrm{H}) ;{ }^{13} \mathbf{C} \mathbf{N M R}$ $\left(\mathbf{1 0 1} \mathbf{~ M H z}, \mathbf{C D C l}_{3}\right) \boldsymbol{\delta} 137.27,120.35,66.01,32.81,31.76,29.11,28.85,22.72,15.33,14.17 ;{ }^{19} \mathbf{F}$ NMR (377 MHz, $\left.\mathbf{C D C l}_{3}\right) \boldsymbol{\delta}-63.17(\mathrm{~d}, J=11.7 \mathrm{~Hz}) ;{ }^{11} \mathbf{B}$ NMR $\left(\mathbf{1 2 8} \mathbf{~ M H z}, \mathbf{C D C l}_{3}\right) \boldsymbol{\delta} 29.15$.

${ }^{1} \mathrm{H} \mathrm{NMR}\left(\mathrm{CDCl}_{3}, 400 \mathrm{MHz}\right)$ of compound 1a
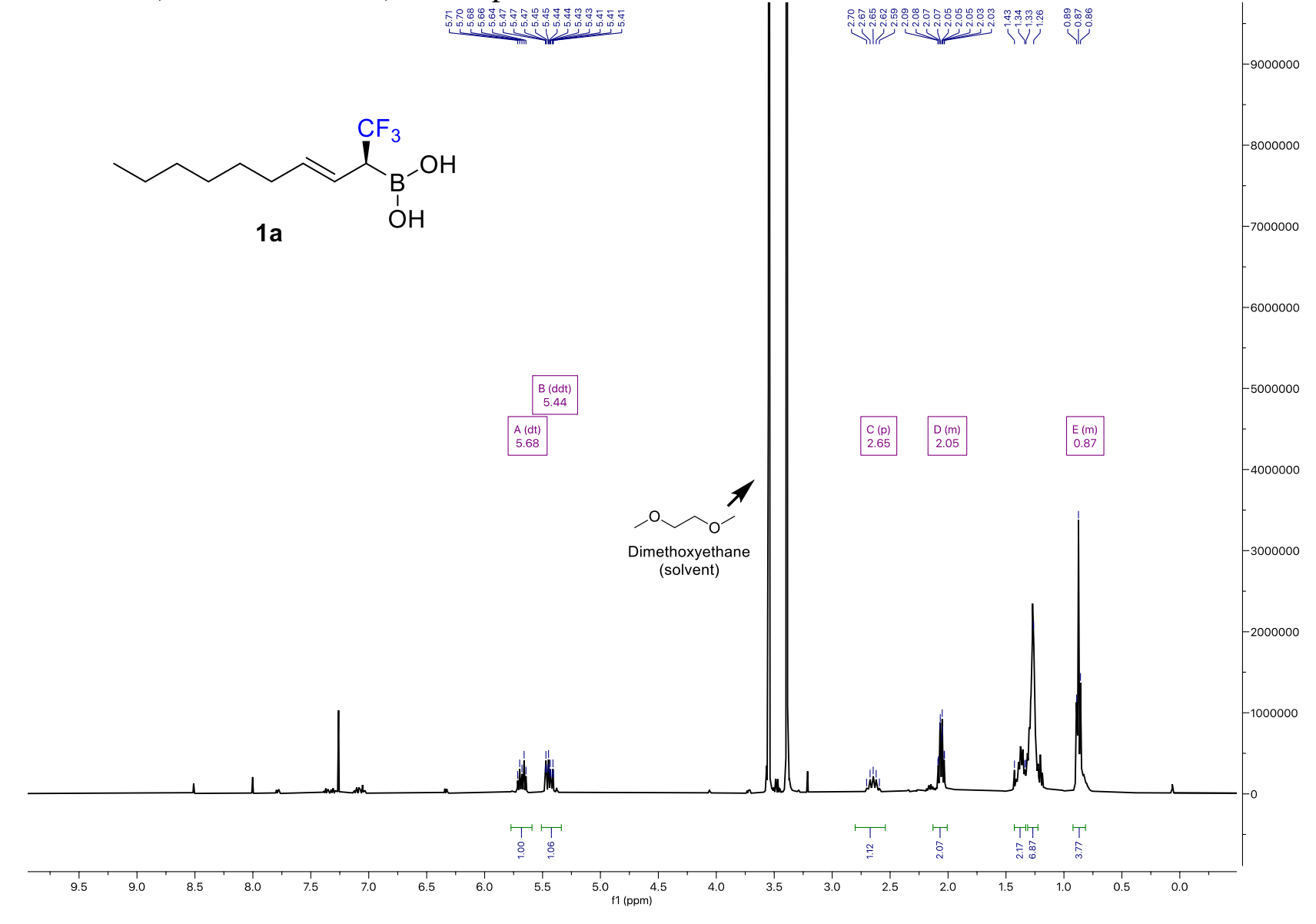
${ }^{13} \mathrm{C} \mathrm{NMR}\left(\mathrm{CDCl}_{3}, 101 \mathrm{MHz}\right)$ of compound $\mathbf{1 a}$
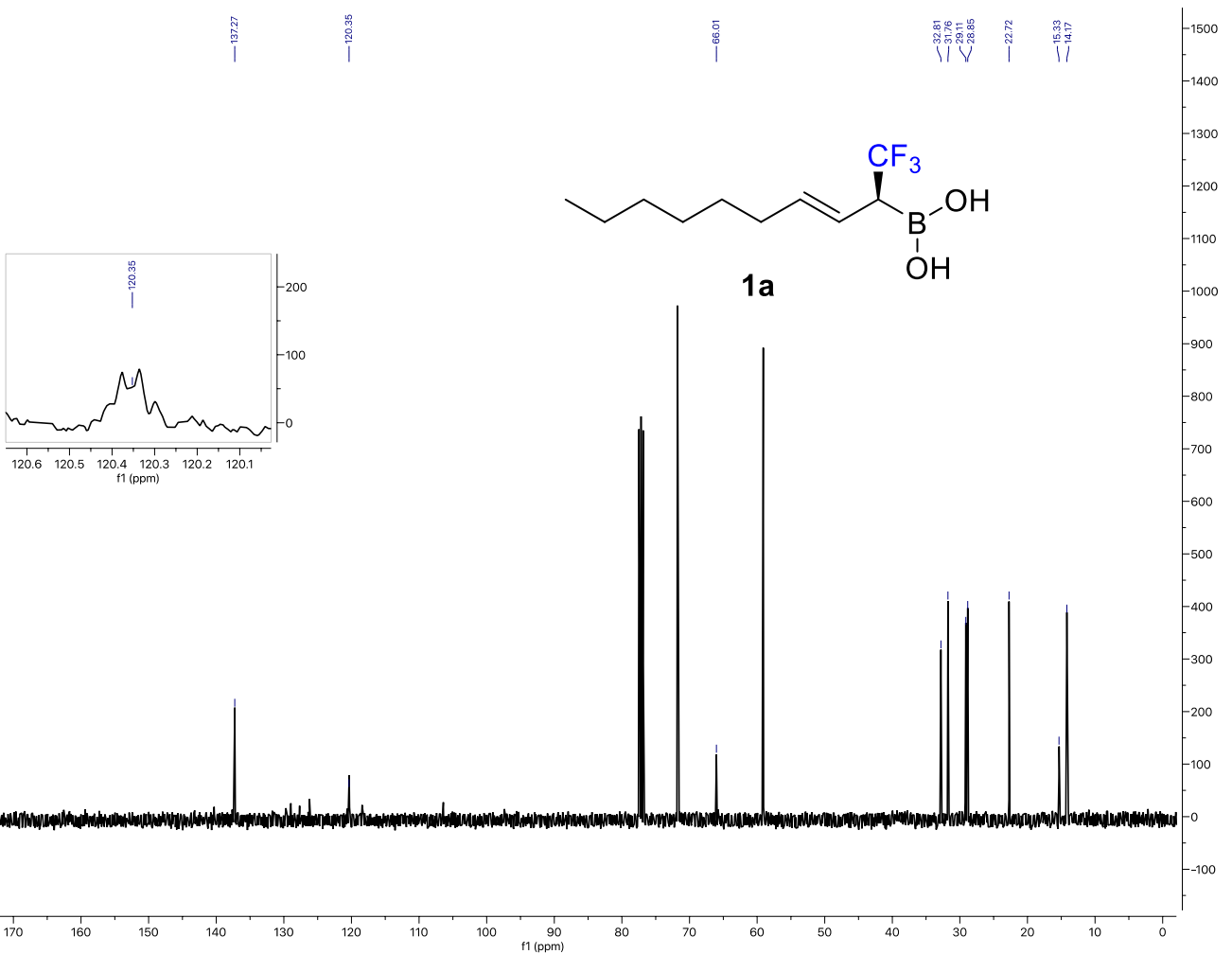

${ }^{19} \mathrm{~F}$ NMR (377 MHz, $\mathrm{CDCl}_{3}$ ) of compound 1a
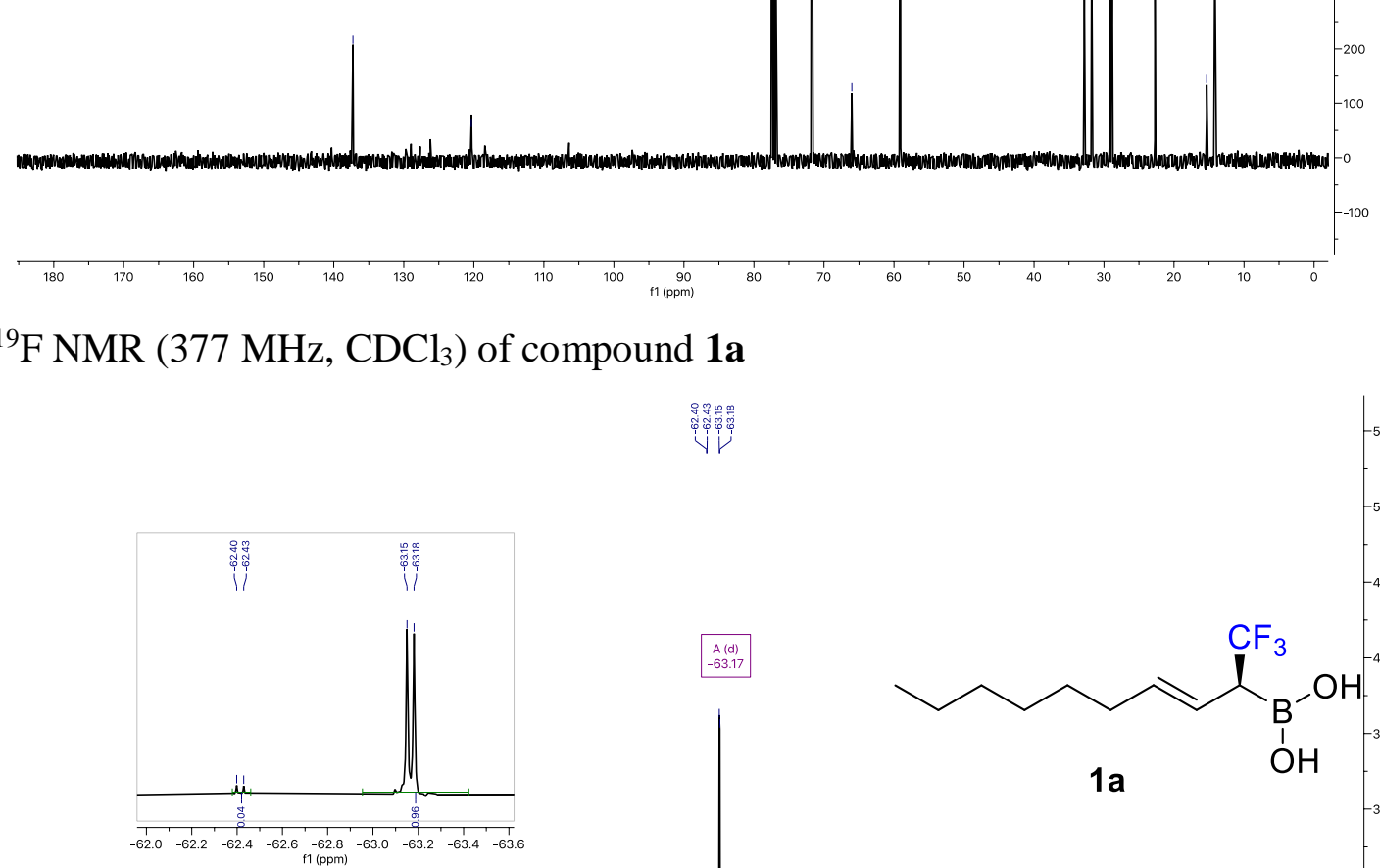

影踥

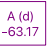
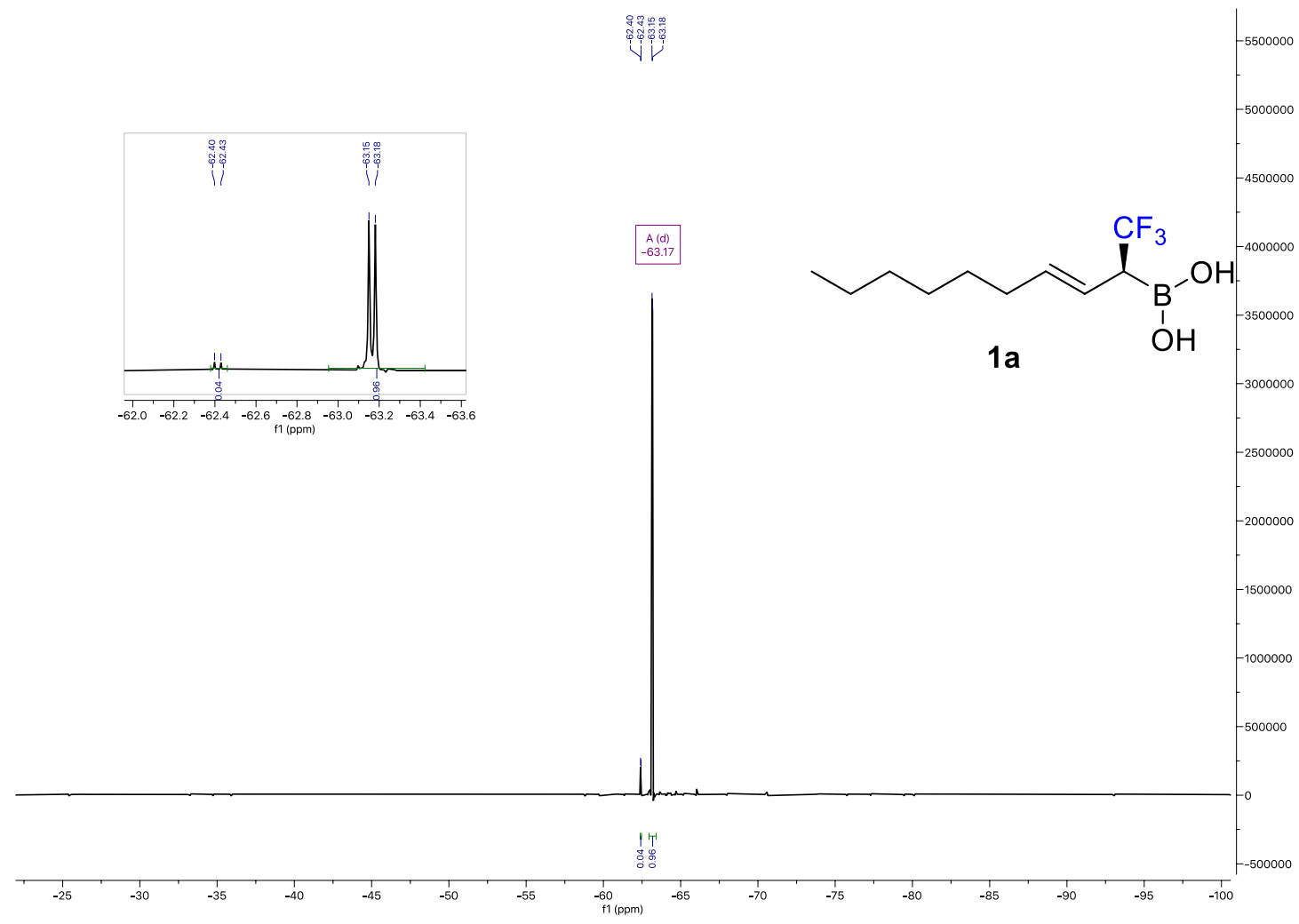
${ }^{11} \mathrm{~B}$ NMR (128 MHz, $\left.\mathrm{CDCl}_{3}\right)$ of compound $\mathbf{1 a}$

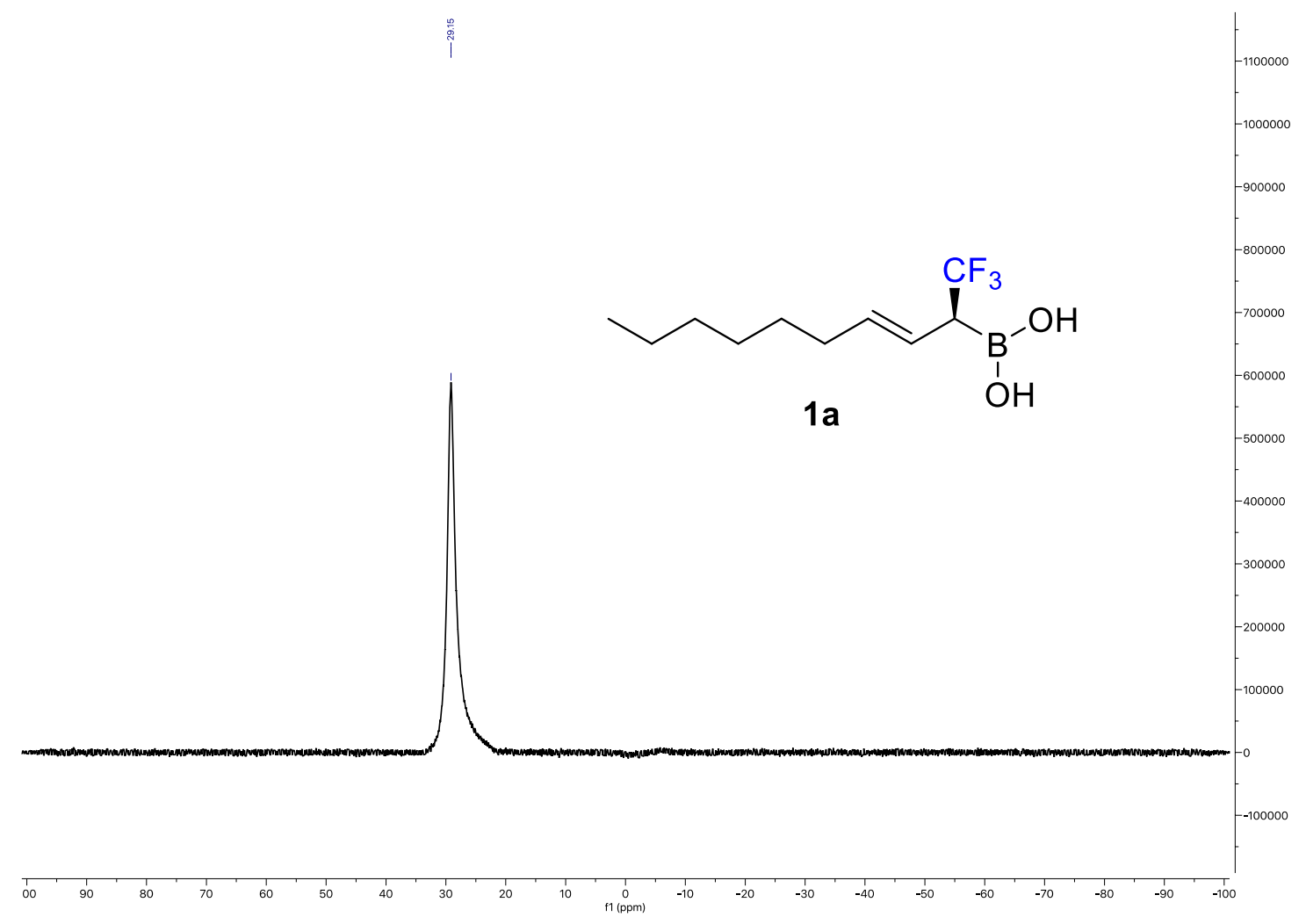


Preparation of the NMR sample for (S,E)-(1,1,1-trifluoro-4-phenylbut-3-en-2-yl)boronic<smiles>OB(O)C(/C=C/c1ccccc1)C(F)(F)F</smiles>
acid (1d): Allyl-Bdan 5d $(0.1 \mathrm{mmol})$ was taken in a screw capped reaction tube under argon. $600 \mu \mathrm{L}$ degassed DME was added followed by $500 \mu \mathrm{L} 3 \mathrm{~N} \mathrm{H}_{2} \mathrm{SO}_{4}$. The mixture was stirred overnight at room temperature. Then, $0.5 \mathrm{M} \mathrm{HCl}$ was added as well as $\mathrm{Et}_{2} \mathrm{O}$ and shaken vigorously. The $\mathrm{Et}_{2} \mathrm{O}$ layer was passed through a phase separator and evaporated. $\mathrm{CDCl}_{3}$ was added and $\mathrm{NMR}$ data was recorded. The conversion of allyl-Bdan $\mathbf{5 d}$ into free boronic acid $\mathbf{1 d}$ was found to be $>99 \%$ based on ${ }^{19} \mathrm{~F}$ NMR analysis by comparison to the signal at $-61.99 \mathrm{ppm}$ that belongs to a $<1 \%$ trace of allyl-Bdan $\mathbf{5 d}$ (See NMR).

${ }^{1} \mathbf{H}$ NMR (400 MHz, CDCl $) \boldsymbol{\delta} 7.40(\mathrm{dt}, J=7.9,1.3 \mathrm{~Hz}, 2 \mathrm{H}), 7.33(\mathrm{tt}, J=6.5,0.9 \mathrm{~Hz}, 2 \mathrm{H}), 7.30$ $-7.22(\mathrm{~m}, 1 \mathrm{H}), 6.60(\mathrm{~d}, J=15.9 \mathrm{~Hz}, 1 \mathrm{H}), 6.24(\mathrm{dd}, J=15.9,9.4 \mathrm{~Hz}, 1 \mathrm{H}), 2.87(\mathrm{p}, J=11.2 \mathrm{~Hz}$, $1 \mathrm{H}) ;{ }^{13} \mathbf{C}$ NMR (101 MHz, $\left.\mathbf{C D C l}_{3}\right) \delta$ 136.64, 135.00, 128.72, 127.96, $127.48\left(\mathrm{q},{ }^{1} J_{C-F}=277.4\right.$ $\mathrm{Hz}), 126.45,120.54\left(\mathrm{q},{ }^{3} J_{C-F}=3.9 \mathrm{~Hz}\right) ;{ }^{19} \mathbf{F}$ NMR $\left(377 \mathbf{M H z}, \mathbf{C D C l}_{3}\right) \boldsymbol{\delta}-62.80(\mathrm{~d}, J=11.5 \mathrm{~Hz})$; ${ }^{11}$ B NMR (128 MHz, $\left.\mathbf{C D C l}_{3}\right) \delta 29.03$.

${ }^{1} \mathrm{H}$ NMR $\left(\mathrm{CDCl}_{3}, 400 \mathrm{MHz}\right)$ of compound $\mathbf{1 d}$

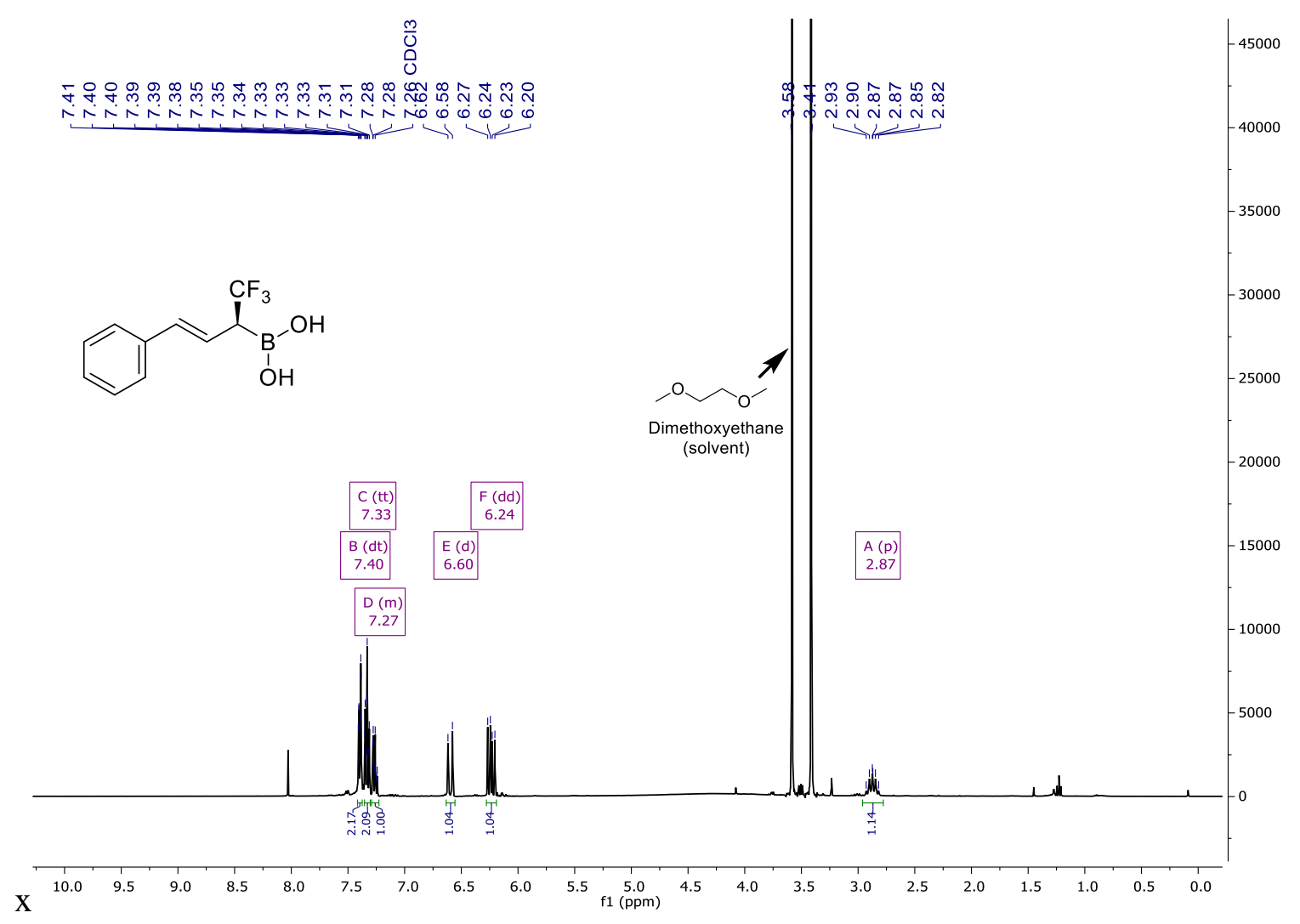


${ }^{13} \mathrm{C}$ NMR $\left(\mathrm{CDCl}_{3}, 101 \mathrm{MHz}\right)$ of compound $\mathbf{1 d}$

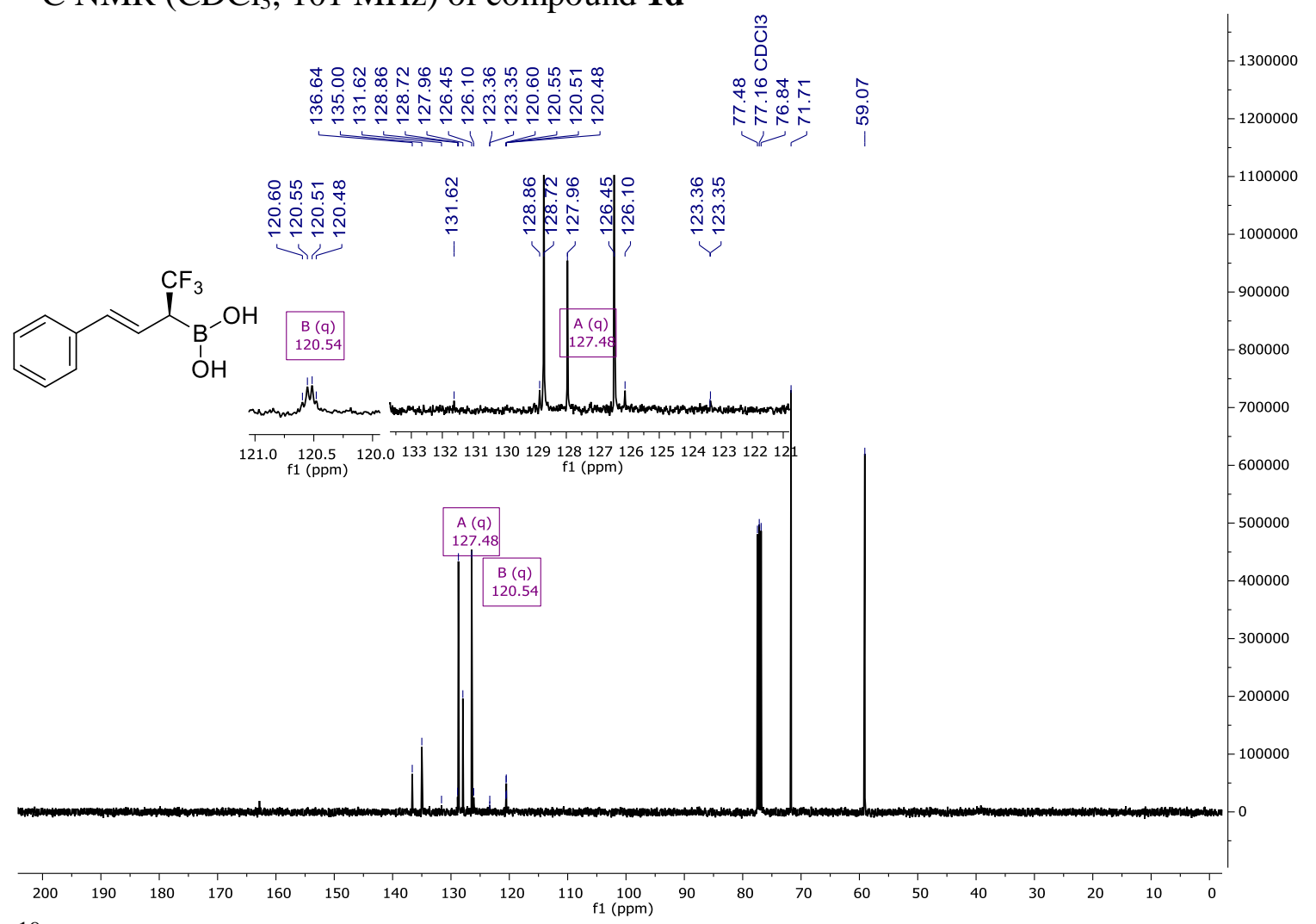

${ }^{19} \mathrm{~F}$ NMR (377 MHz, $\mathrm{CDCl}_{3}$ ) of compound 1d

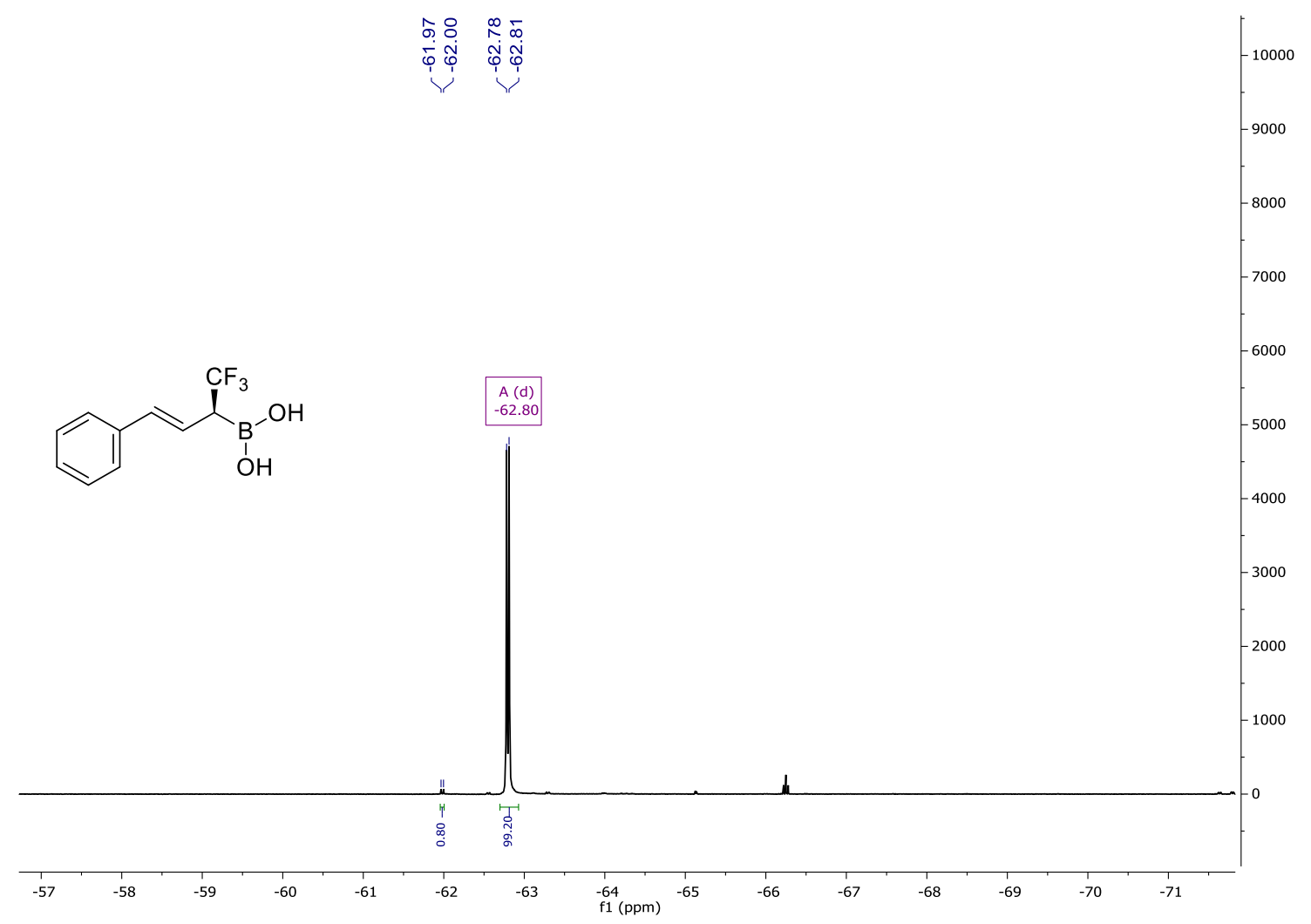


${ }^{11} \mathrm{~B}$ NMR (128 MHz, $\left.\mathrm{CDCl}_{3}\right)$ of compound $\mathbf{1 d}$

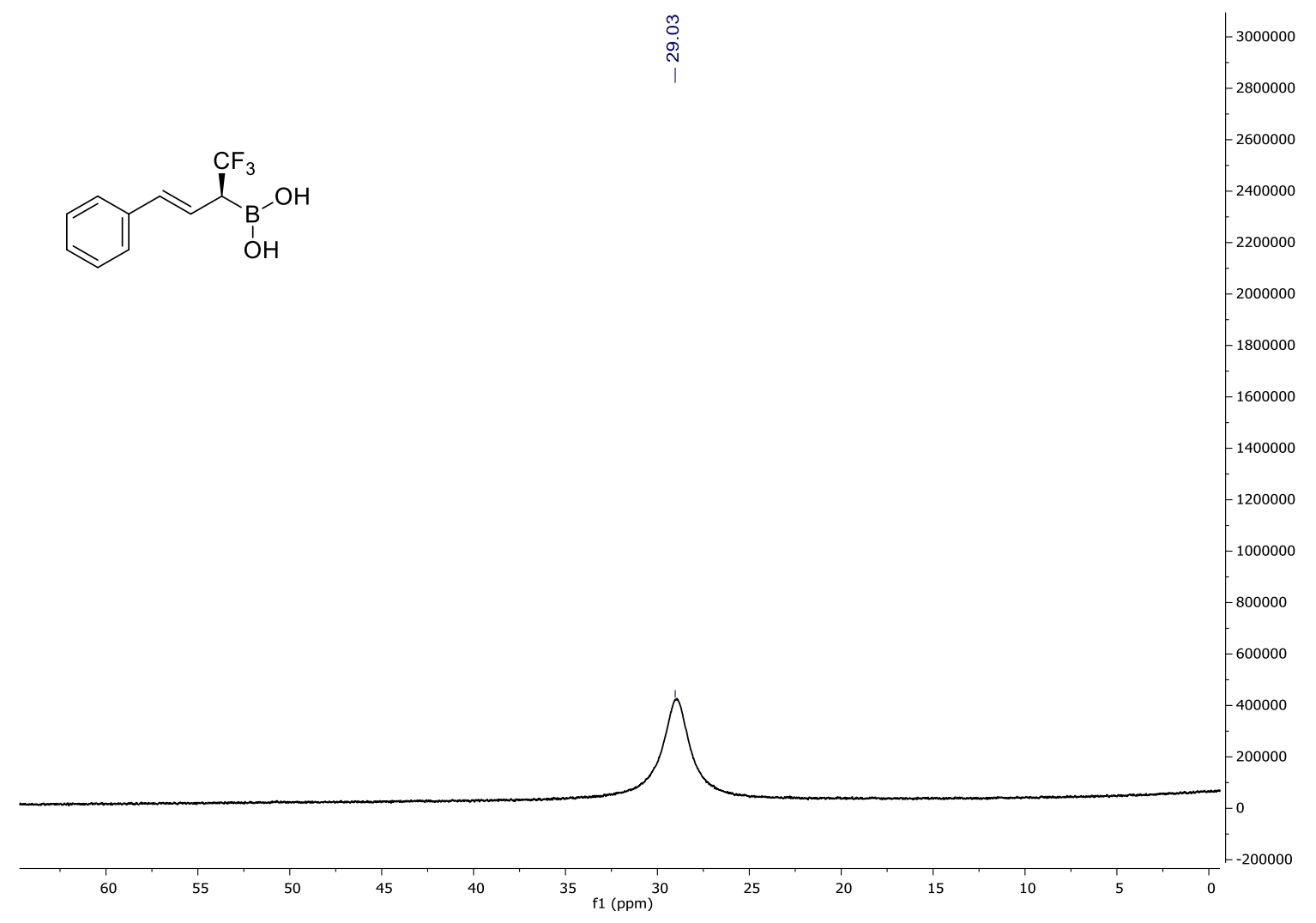




\section{Studies of the effects of molecular sieves on purified allylboronic acid 1a in toluene- $d 8$}

In these studies, we used toluene- $d 8$ as solvent because of three reasons: i) toluene is the reaction medium of the allylboration reactions with purified allylboronic acids given in Table 2; ii) toluene$d 8$ can be more efficiently dried by molecular sieves than $\mathrm{CDCl}_{3}$ and iii) in our experience airsensitive dehydrated allylboronic acids are more stable in toluene- $d 8$ than in $\mathrm{CDCl}_{3}$.

Preparation of the NMR sample for (S,E)-(1,1,1-trifluorodec-3-en-2-yl)boronic acid (1a) in<smiles>CCCCCC/C=C/[C@H](B(O)O)C(F)(F)F</smiles>
toluene-d8: Allyl-Bdan 5a $(0.1 \mathrm{mmol})$ was taken in a screw capped reaction tube under argon. Then, $600 \mu \mathrm{L}$ degassed DME was added followed by $500 \mu \mathrm{L} 3 \mathrm{~N} \mathrm{H}_{2} \mathrm{SO}_{4}$. The mixture was stirred overnight at room temperature. Then, $0.5 \mathrm{M} \mathrm{HCl}$ was added as well as toluene- $d 8$ and shaken vigorously. The toluened8 layer was passed through a phase separator and the NMR spectrum was recorded. The conversion of allyl-Bdan 5a into free boronic acid 1a was found to be $93 \%$ based on ${ }^{19} \mathrm{~F}$ NMR analysis by comparison to the signal at -62.57 ppm that belongs to a $7 \%$ of allyl-Bdan 5a (see NMR spectrum bellow).

${ }^{1}$ H NMR (400 MHz, Toluene-d8) $\delta 6.61(\mathrm{~s}, 2 \mathrm{H}), 5.74-5.54(\mathrm{~m}, 2 \mathrm{H}), 2.74(\mathrm{qd}, J=11.6,8.7 \mathrm{~Hz}$, 1H), $1.98-1.79(\mathrm{~m}, 2 \mathrm{H}), 1.32-1.04(\mathrm{~m}, 8 \mathrm{H}), 0.84(\mathrm{t}, J=7.0 \mathrm{~Hz}, 3 \mathrm{H}) .{ }^{19} \mathbf{F}$ NMR (377 MHz, Toluene-d8) $\delta-63.08(\mathrm{~d}, J=11.6 \mathrm{~Hz})$.

${ }^{1} \mathrm{H}$ NMR (400 MHz) of allyl boronic acid 1a in toluene- $d 8$

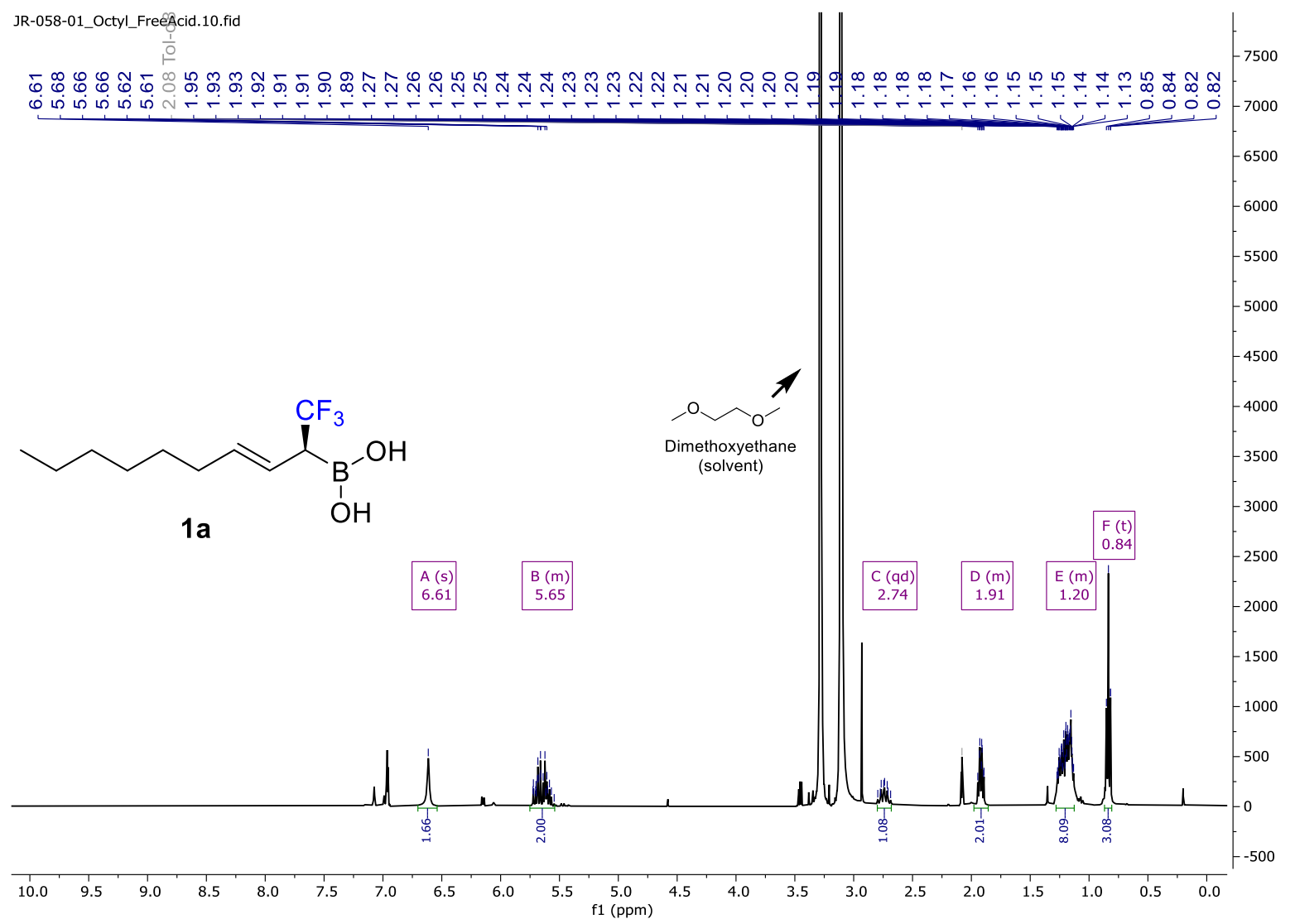


${ }^{1} \mathrm{H}$ NMR of boronic acid $1 \mathrm{a}$ in toluene-d8 after 1 hour drying over molecular sieves: After addition $(1 \mathrm{~h})$ of molecular sieves to the toluene- $d 8$ sample of free chiral boronic acid 1a significant changes were observed in the 1H-NMR spectrum (see below). The singlet at 6.61 (tentatively assigned to the proton of the $\mathrm{B}(\mathrm{OH})_{2}$ group) substantially reduced. In addition, the shape of the alkenyl protons in the range of at 5.5-5.7 were radically changed. We conclude that these changes upon addition of molecular sieves to boronic acid 1a indicate formation of the boroxine form and other anhydrides, which are possibly involved in an equilibrium process.

b) After 1 hour drying with molecular sieves

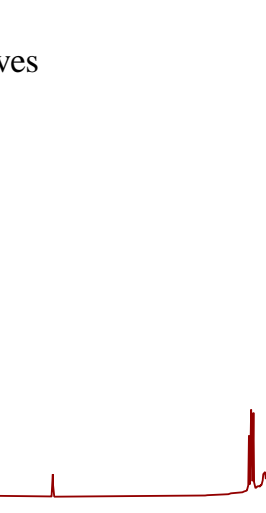

a) Free chiral allyl boronic acid $\mathbf{1 a}$
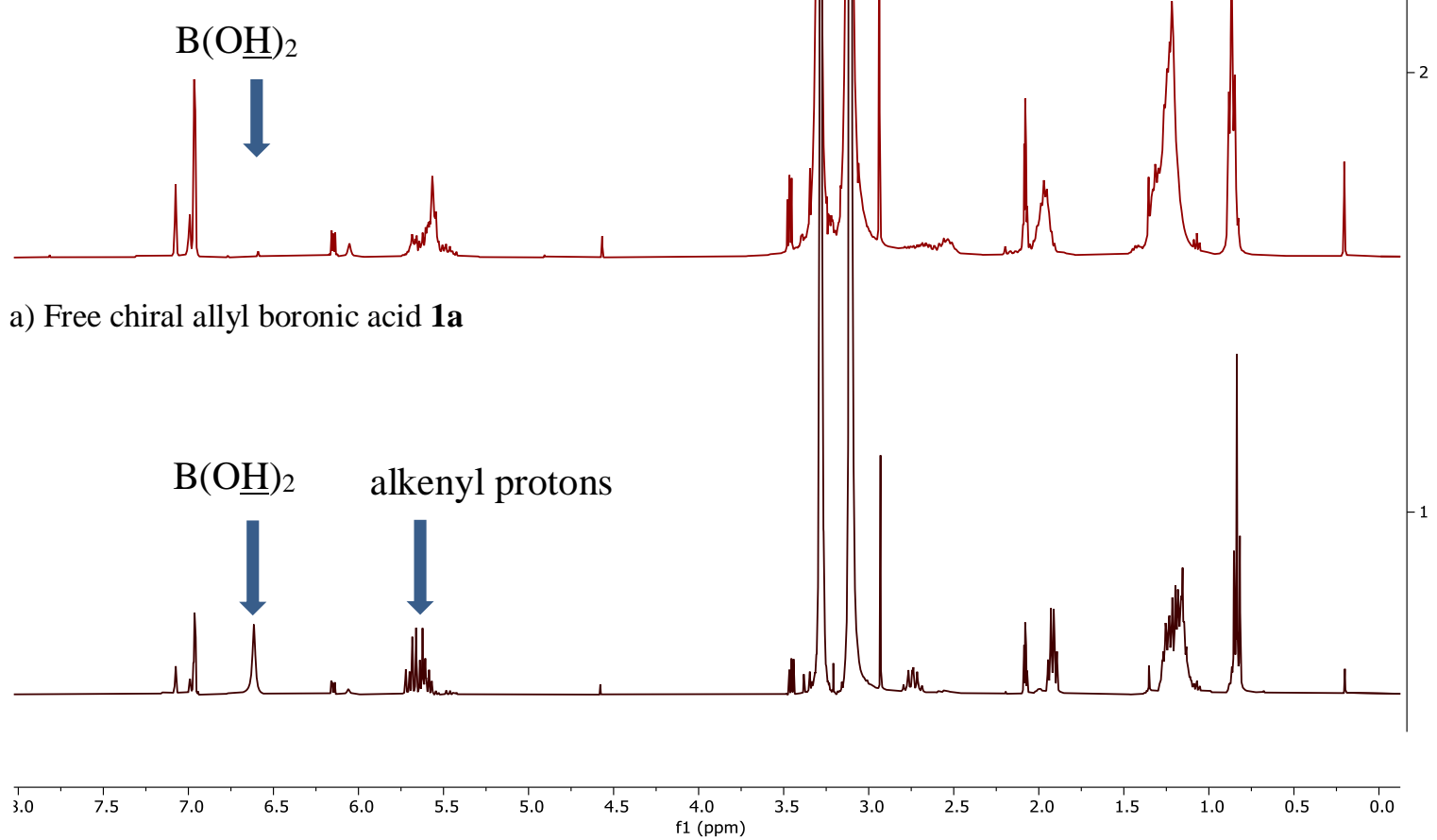
${ }^{19}$ F NMR (377 MHz) of allyl boronic acid 1a in toluene-d8: The doublet at -63.1 was assigned to the signals of the $\mathrm{CF}_{3}$ group of allyl boronic acid 1a and the signal at -62.6 belongs to the $\mathrm{CF}_{3}$ group of unreacted allylBdan compound 5a. According to integration of the peak areas, 1a was formed in $93 \%$ conversion by hydrolysis of $\mathbf{5 a}$.

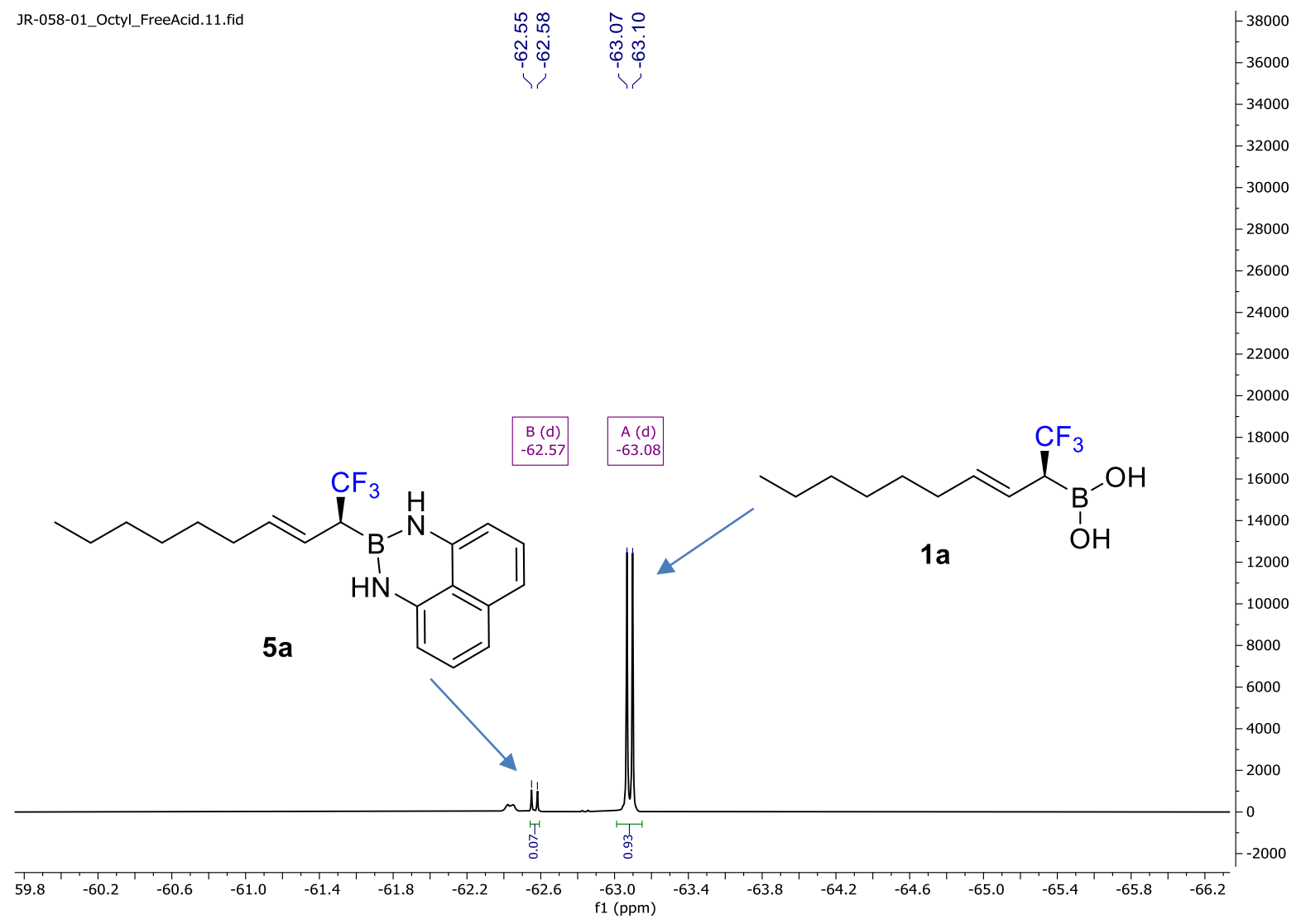


${ }^{19} \mathrm{~F}$ NMR of allyl boronic acid $1 \mathrm{a}$ in toluene- $d 8$ after drying with molecular sieves $(1 \mathrm{~h}$ and 18 h): After drying the sample of allylboronic acid la over molecular sieves $(1-18 \mathrm{~h})$ the ${ }^{19} \mathrm{~F}$ signal at $-62.6(d)$, assigned to allylBdan 5a, remained unchanged. However, the peak at -63.1 (d) assigned to allylboronic acid 1a underwent significant changes on addition of molecular sieves, such as broadening and change of the line shape. These changes confirm our above conclusion based on the analysis of the ${ }^{1} \mathrm{H}$ NMR spectrum. The changes indicate dehydration of 1a by formation of equilibrium mixture of the boroxine form (1a-boroxine) and other anhydrides of 1a. As mentioned above dehydrated 1a is a highly reactive but relatively unstable species. The appearance of the broad peak at -60.1 can be attributed to formation of decomposition product(s) over time.
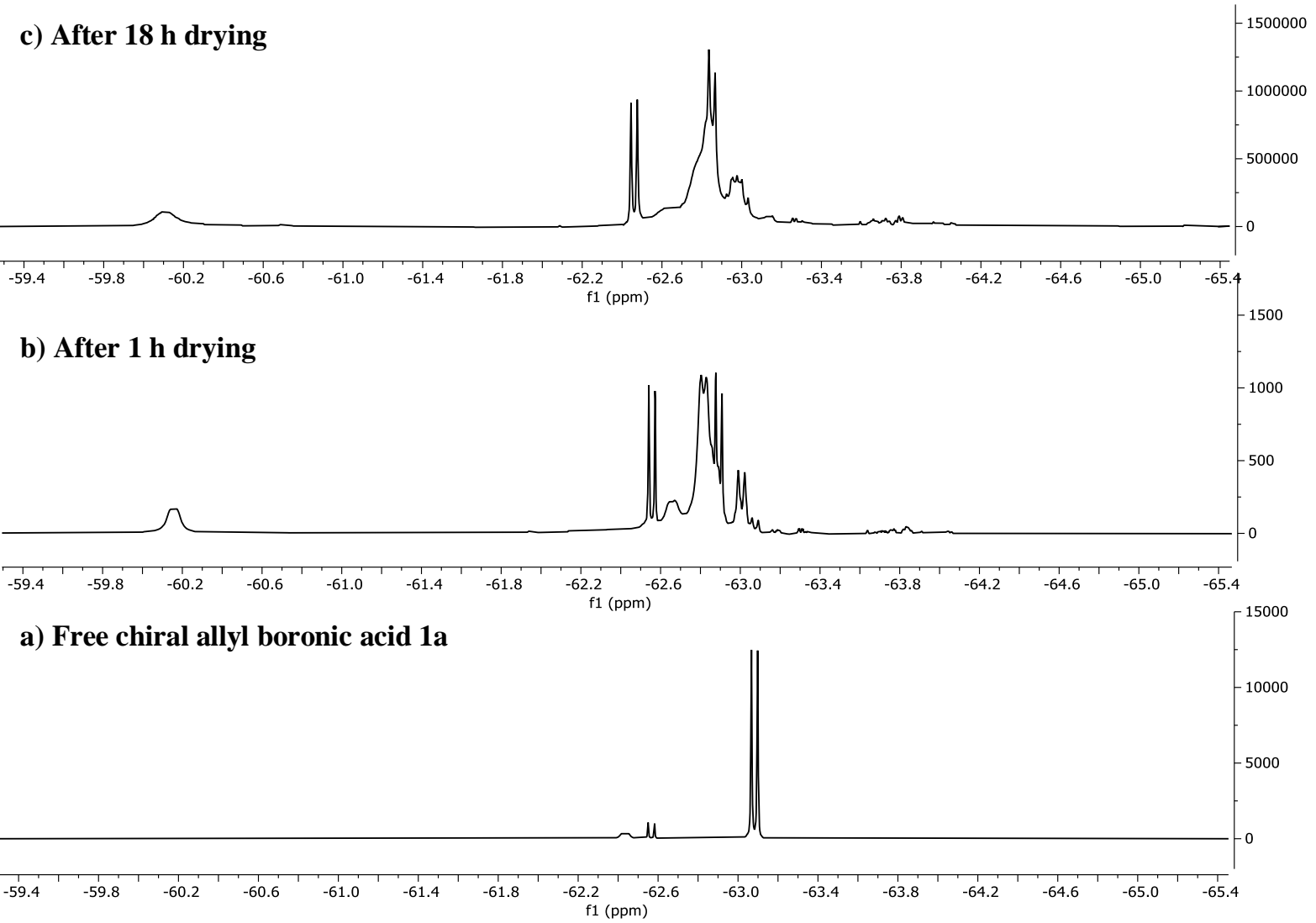


\section{References}

(1) Pisella, G.; Gagnebin, A.; Waser, J.; Pisella, G.; Gagnebin, A.; Waser, J. Copper-Catalyzed Insertion of Diazo Compounds into Vinyl Hypervalent Iodine Reagents to Generate Allylic Esters Vinyl Hypervalent Iodine Reagents to Generate Allylic Esters. 2019, No. 1. https://doi.org/10.26434/chemrxiv.7892513.v1.

(2) Argintaru, O. A.; Ryu, D.; Aron, I.; Molander, G. A. Synthesis and Applications of $\alpha$ Trifluoromethylated Alkylboron Compounds. Angew. Chem. Int. Ed. 2013, 52 (51), 1365613660. https://doi.org/10.1002/anie.201308036.

(3) Milburn, R. R.; Hussain, S. M. S.; Prien, O.; Ahmed, Z.; Snieckus, V. 3,3'-Dipyridyl BINOL Ligands. Synthesis and Application in Enantioselective Addition of Et2Zn to Aldehydes. Org. Lett. 2007, 9 (22), 4403-4406. https://doi.org/10.1021/o1071276f.

(4) Hoye, T. R.; Jeffrey, C. S.; Shao, F. Mosher Ester Analysis for the Determination of Absolute Configuration of Stereogenic (Chiral) Carbinol Carbons. Nat. Protoc. 2007, 2 (10), 2451-2458. https://doi.org/10.1038/nprot.2007.354.

(5) Brauns, M.; Mantel, M.; Schmauck, J.; Guder, M.; Breugst, M.; Pietruszka, J. Highly Enantioselective Allylation of Ketones: An Efficient Approach to All Stereoisomers of Tertiary Homoallylic Alcohols. Chem. Eur. J. 2017, 23 (50), 12136-12140. https://doi.org/10.1002/chem.201701740. 


\section{NMR Spectra}

${ }^{1} \mathrm{H} \mathrm{NMR}\left(\mathrm{CDCl}_{3}, 400 \mathrm{MHz}\right)$ of compound 1a-Bpin

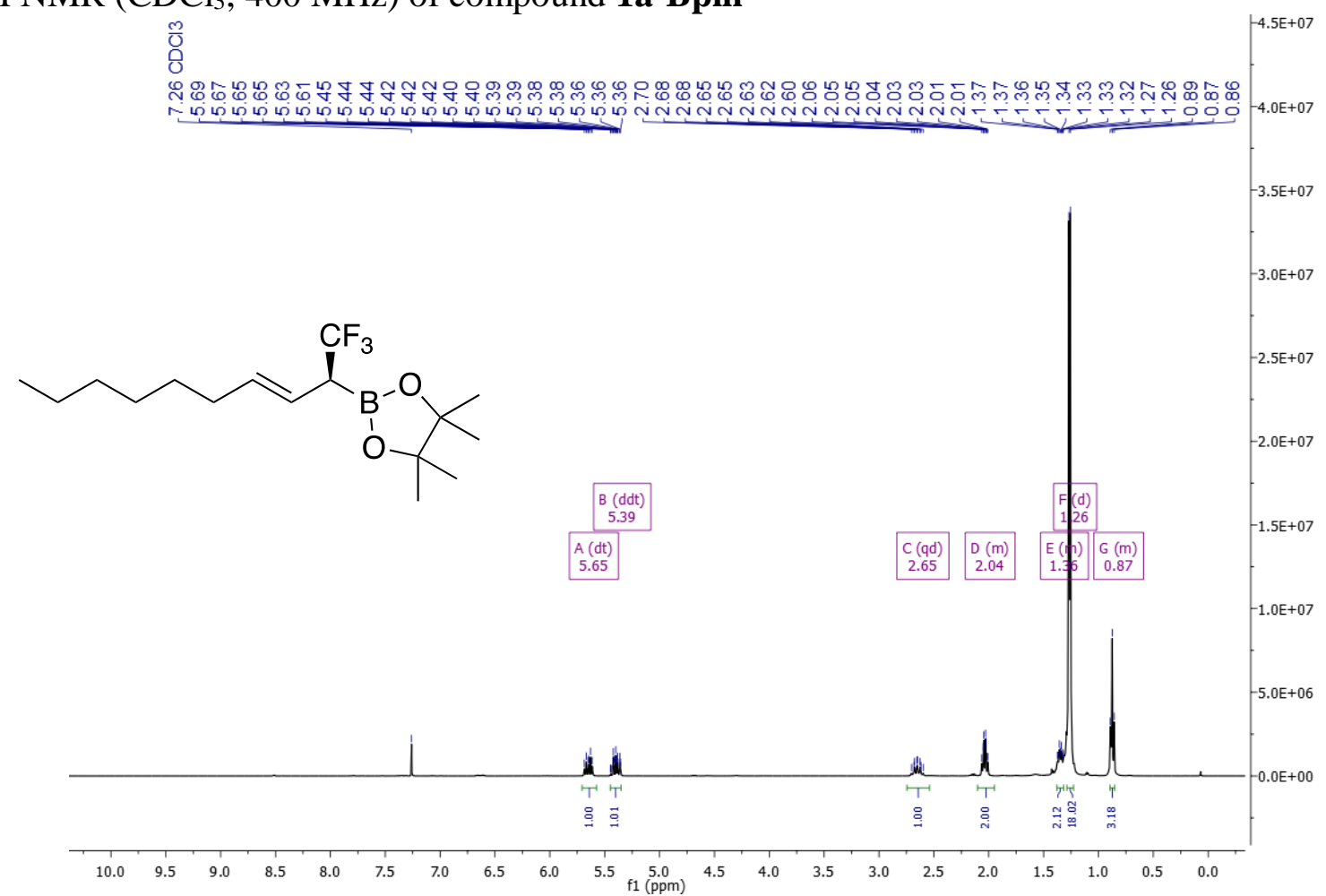

${ }^{13} \mathrm{C}$ NMR $\left(\mathrm{CDCl}_{3}, 101 \mathrm{MHz}\right)$ of compound 1a-Bpin

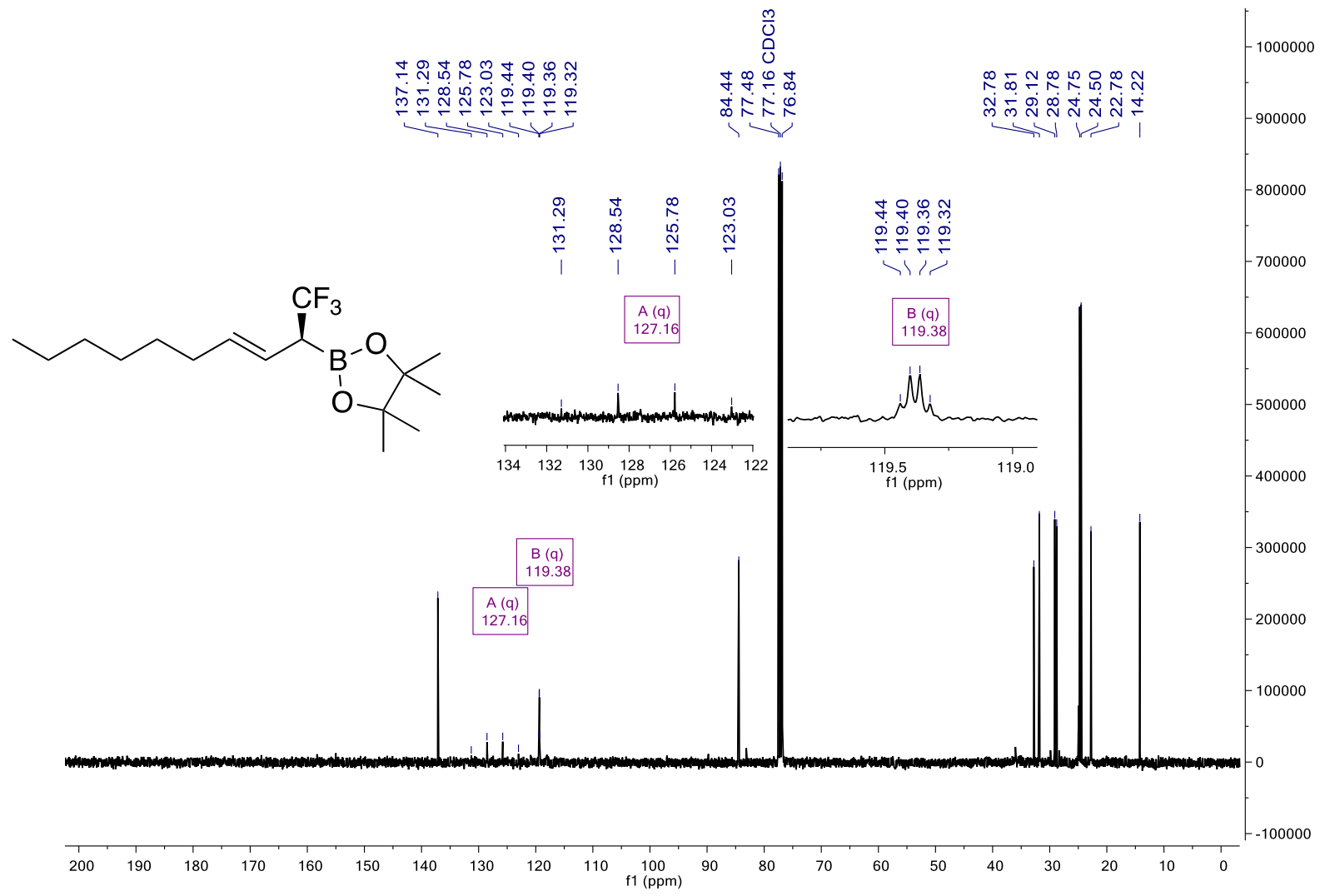


${ }^{19} \mathrm{~F}$ NMR $\left(\mathrm{CDCl}_{3}, 377 \mathrm{MHz}\right)$ of compound 1a-Bpin

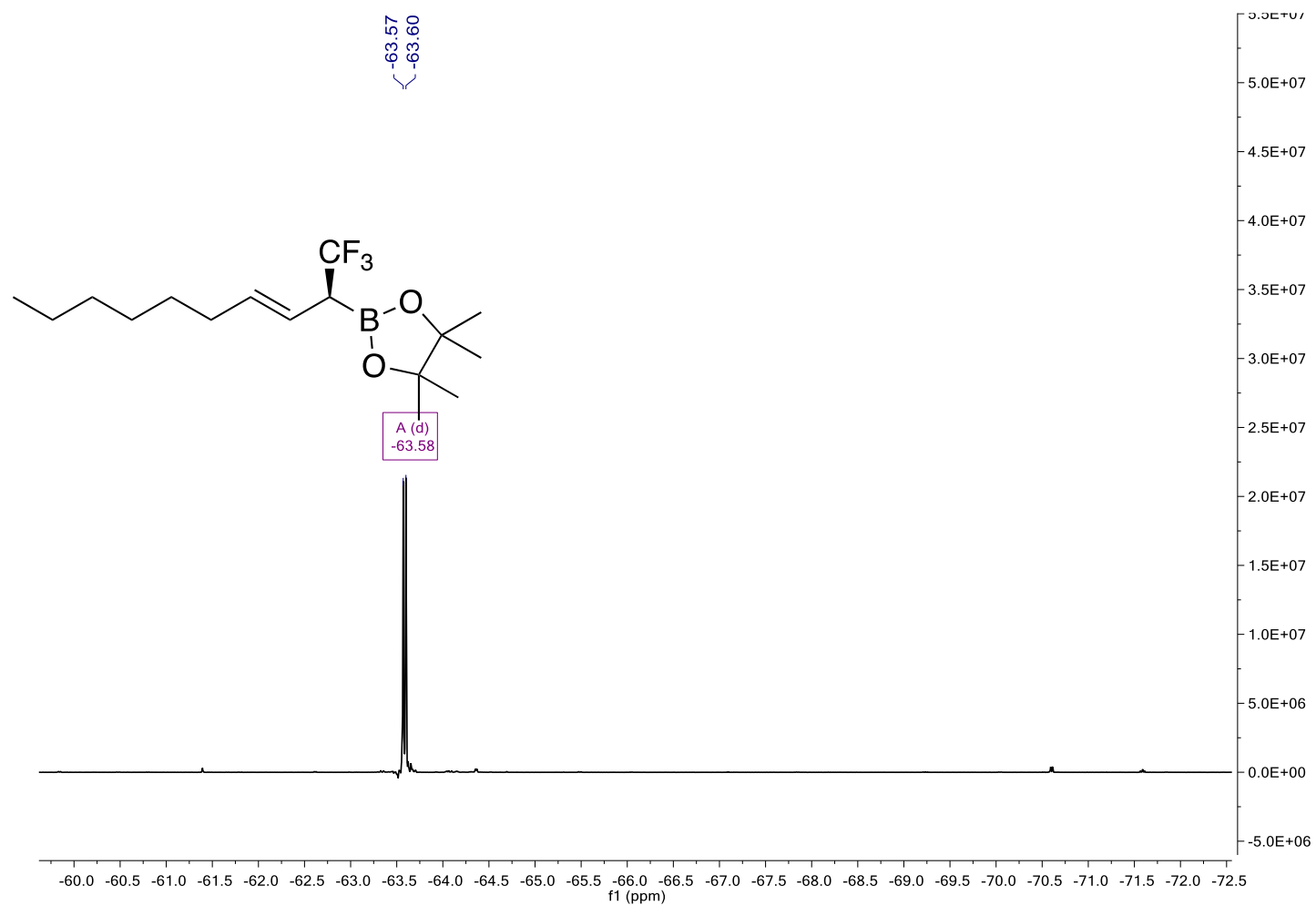

${ }^{11} \mathrm{~B} \mathrm{NMR}\left(\mathrm{CDCl}_{3}, 128 \mathrm{MHz}\right)$ of compound 1a-Bpin

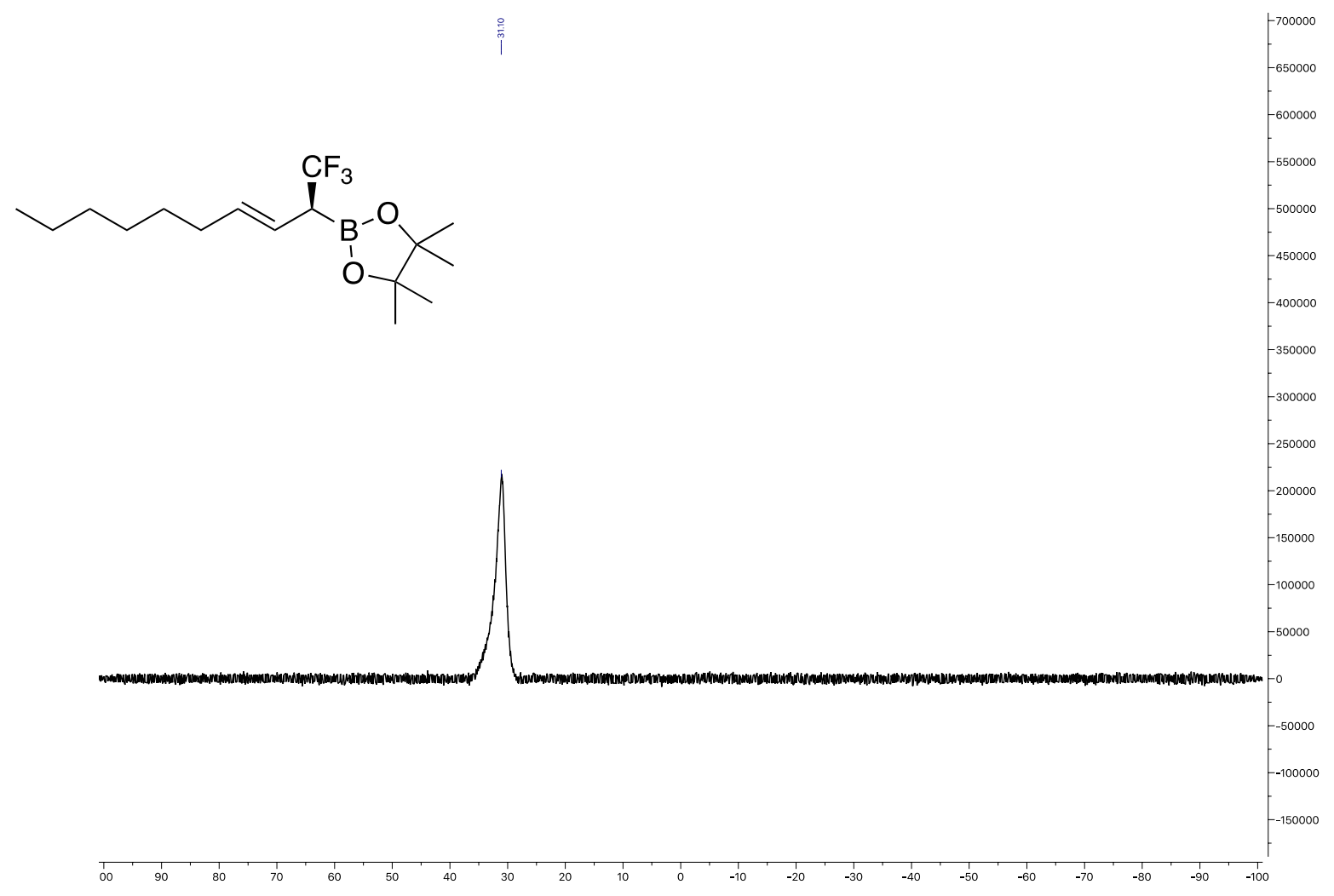

윻 
${ }^{1} \mathrm{H} \mathrm{NMR}\left(\mathrm{CDCl}_{3}, 400 \mathrm{MHz}\right)$ of compound 1a-Bpinane

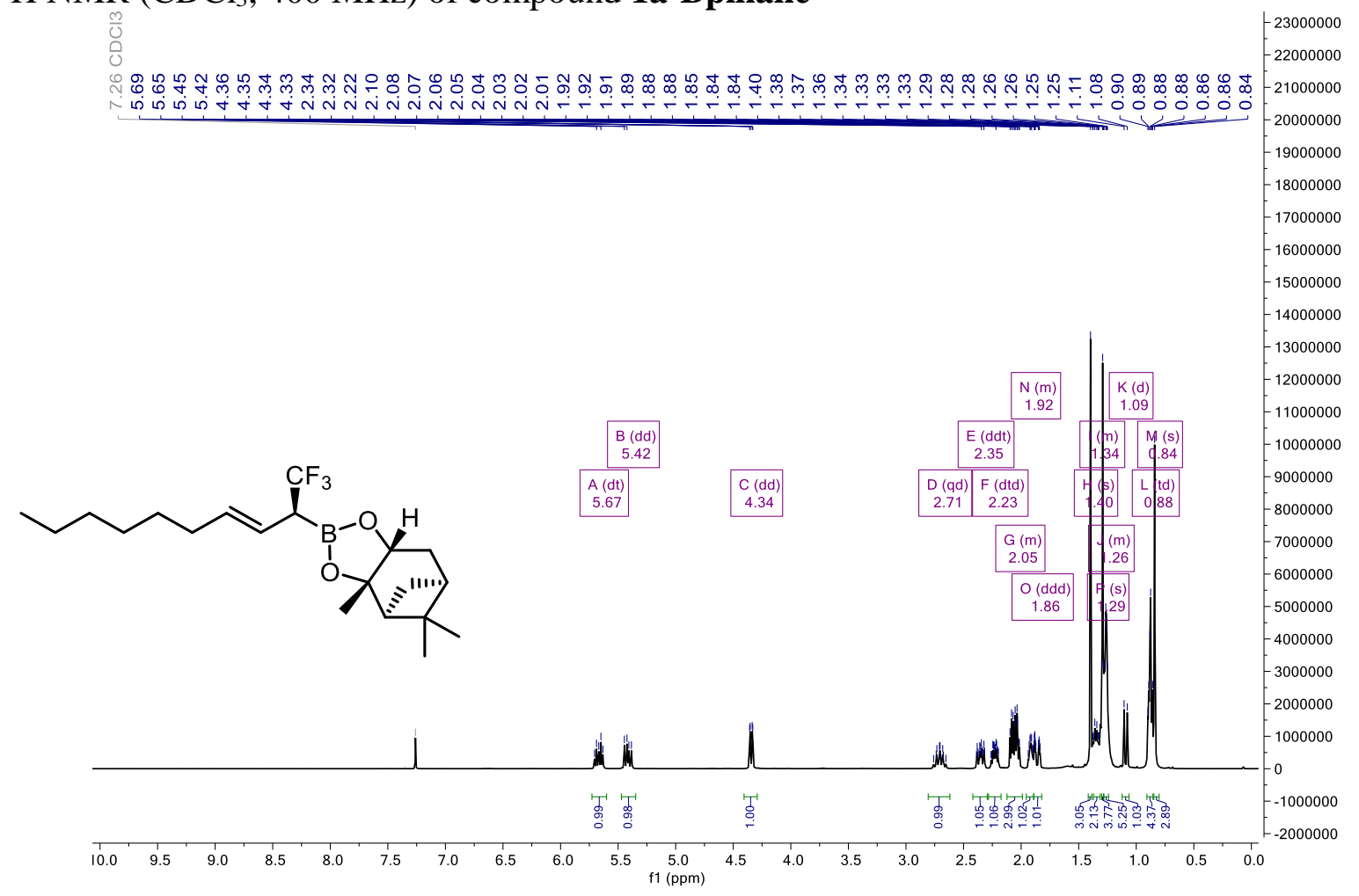

${ }^{13} \mathrm{C} \mathrm{NMR}\left(\mathrm{CDCl}_{3}, 101 \mathrm{MHz}\right)$ of compound 1a-Bpinane

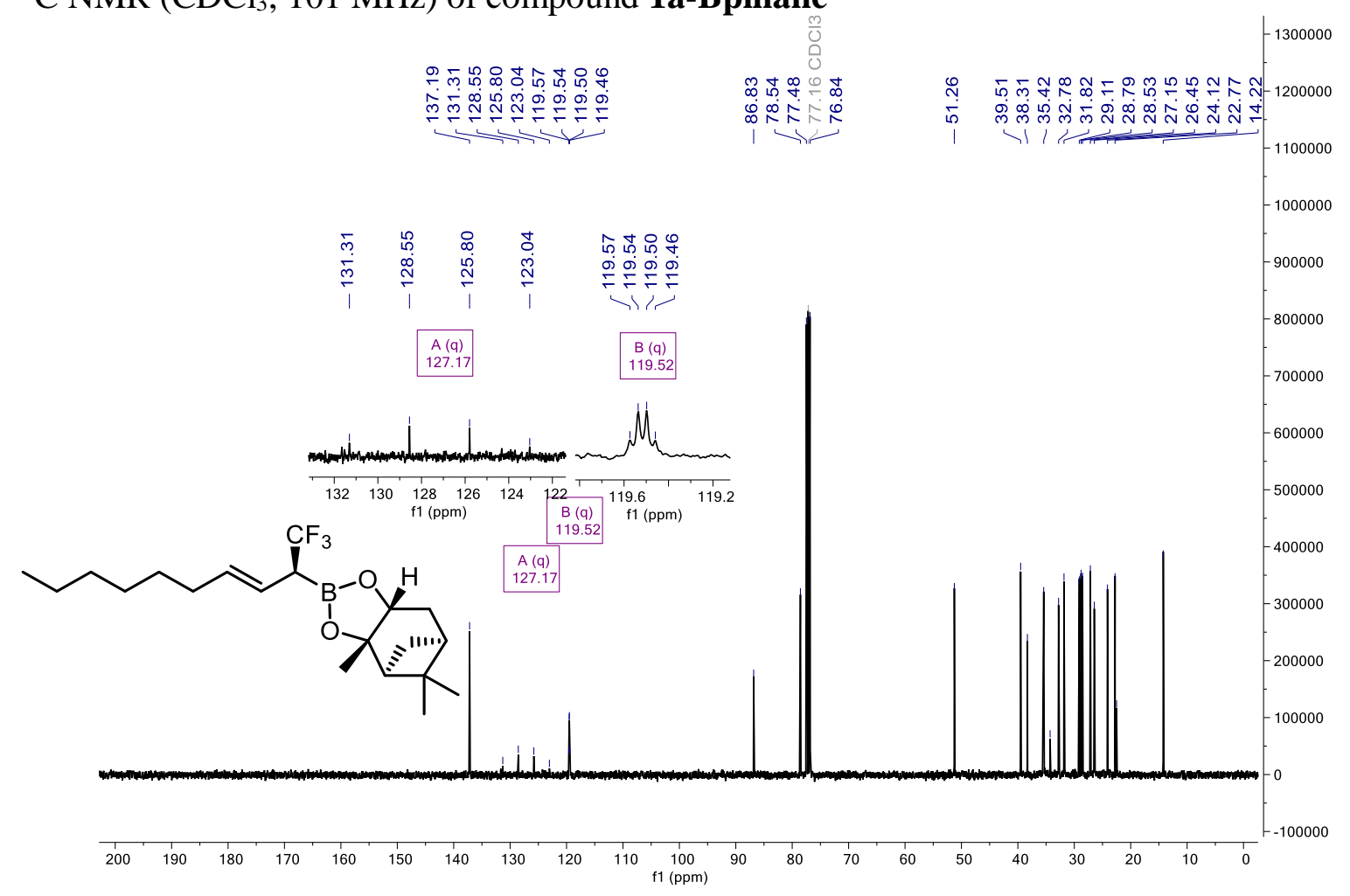


${ }^{19} \mathrm{~F} \mathrm{NMR}\left(\mathrm{CDCl}_{3}, 377 \mathrm{MHz}\right)$ of compound 1a-Bpinane

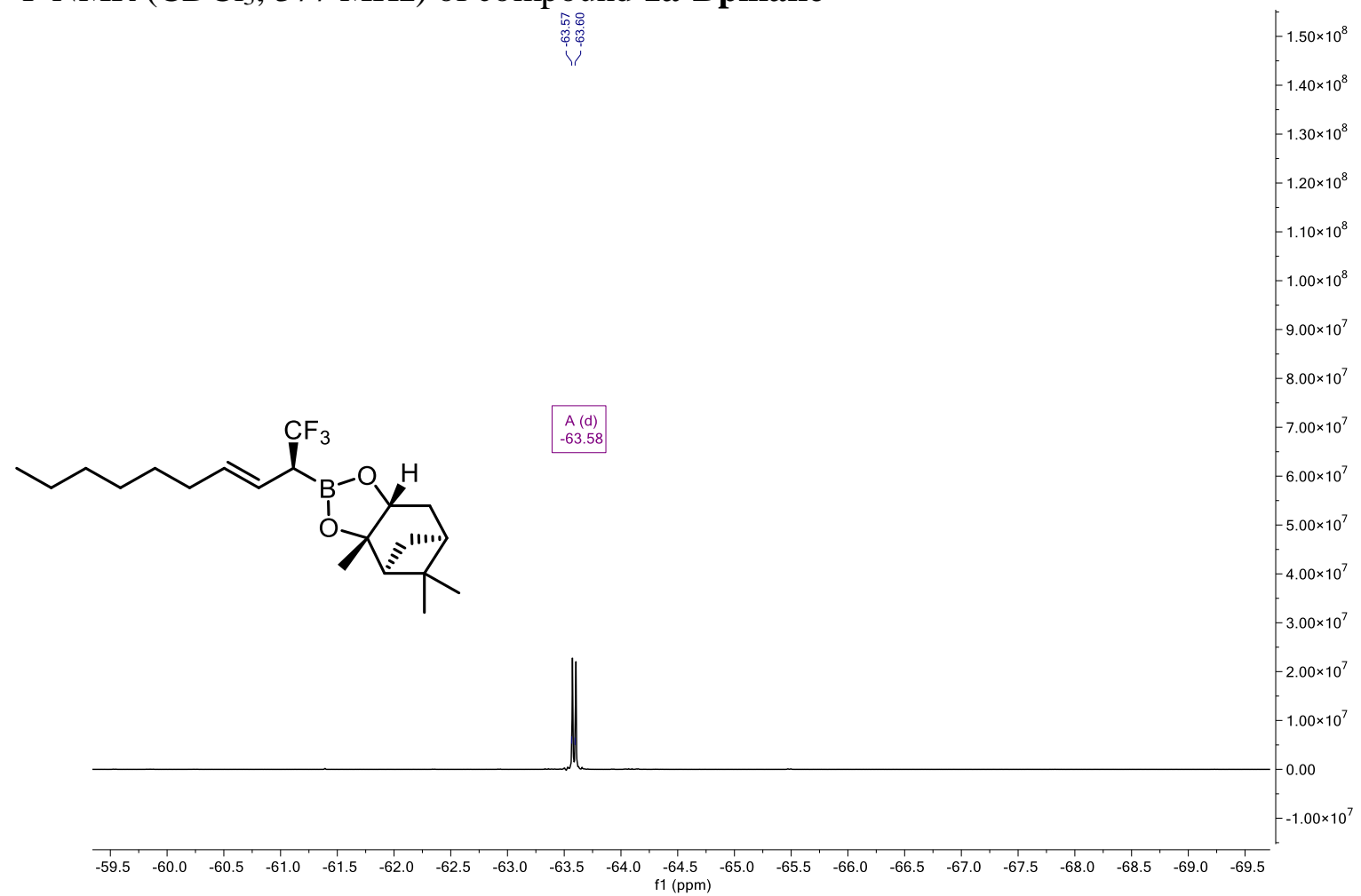

${ }^{11} \mathrm{~B} \mathrm{NMR}\left(\mathrm{CDCl}_{3}, 128 \mathrm{MHz}\right)$ of compound 1a-Bpinane

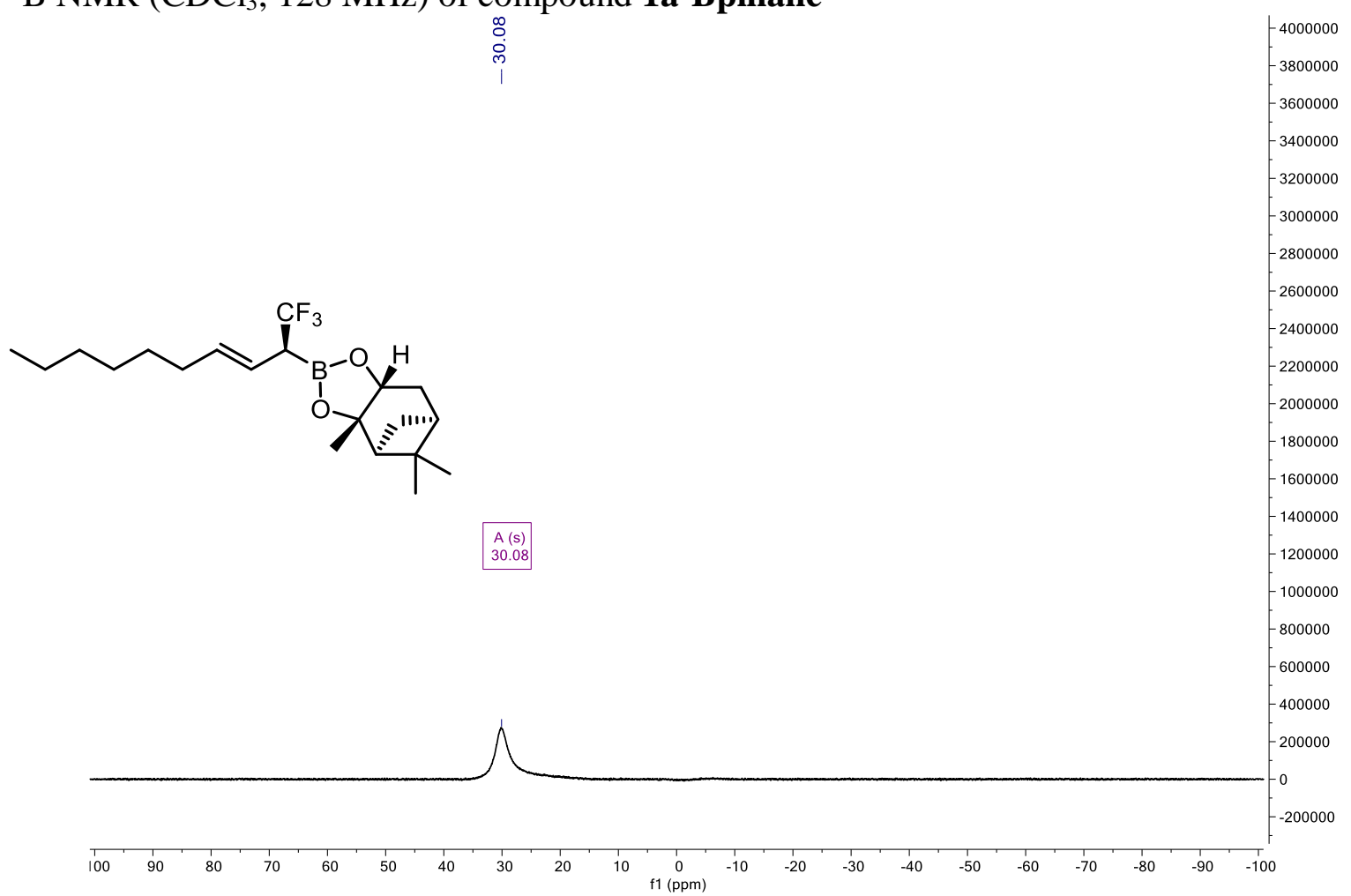




\section{${ }^{1} \mathrm{H}$ NMR $\left(\mathrm{CDCl}_{3}, 400 \mathrm{MHz}\right)$ of compound 5a}

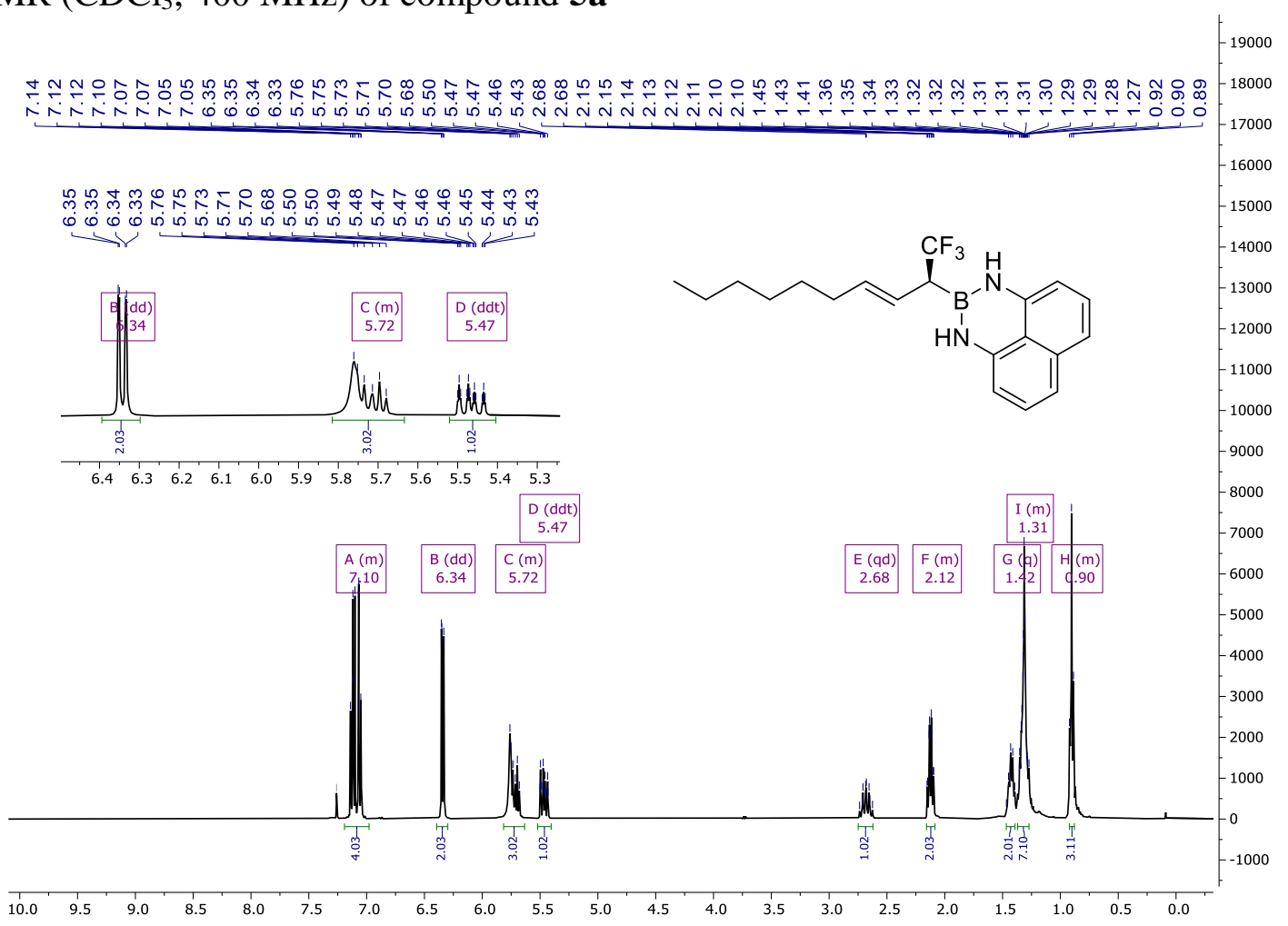

${ }^{13} \mathrm{C} \mathrm{NMR}\left(\mathrm{CDCl}_{3}, 101 \mathrm{MHz}\right)$ of compound $\mathbf{5 a}$

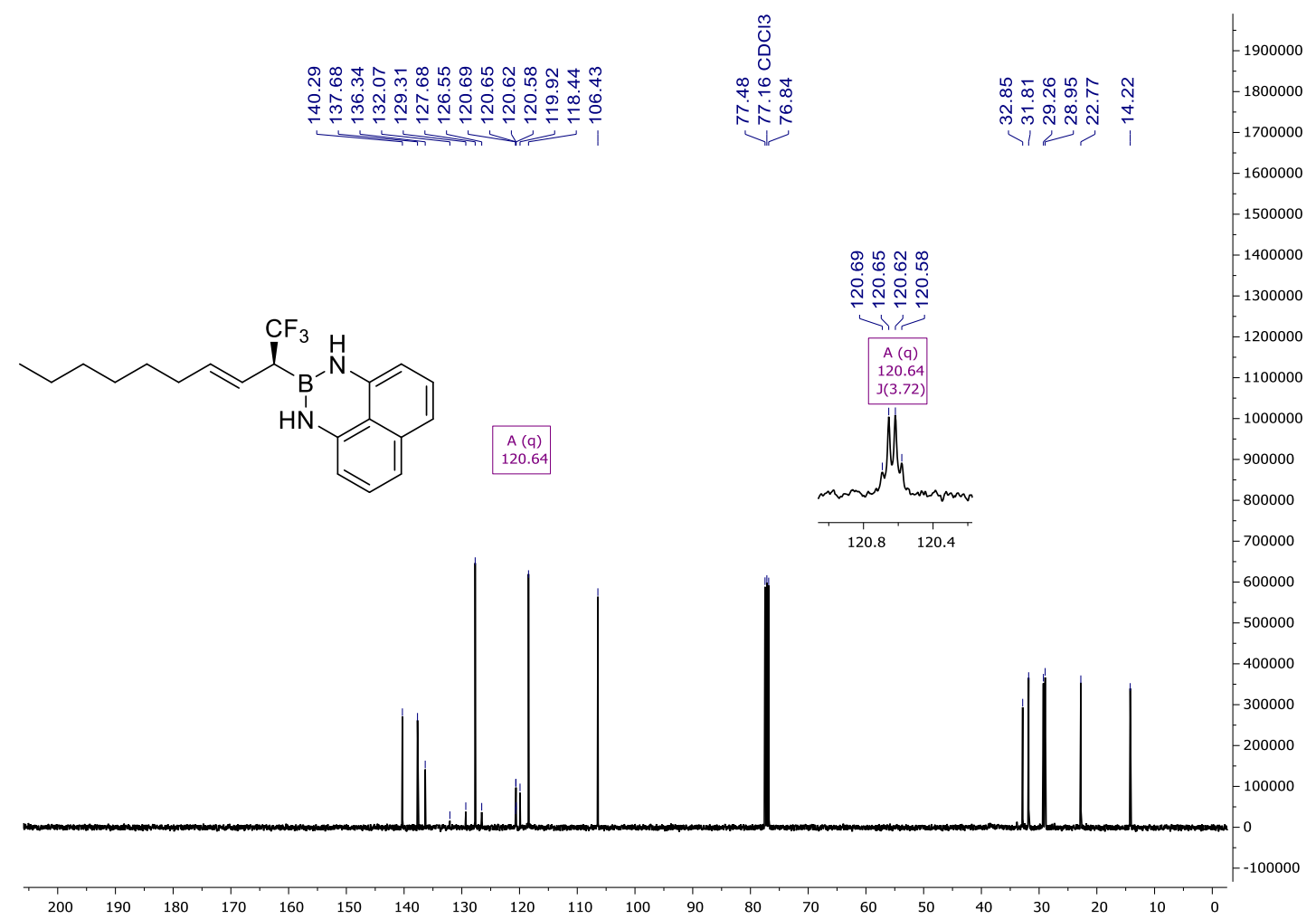


HSQC of compound $\mathbf{5 a}$

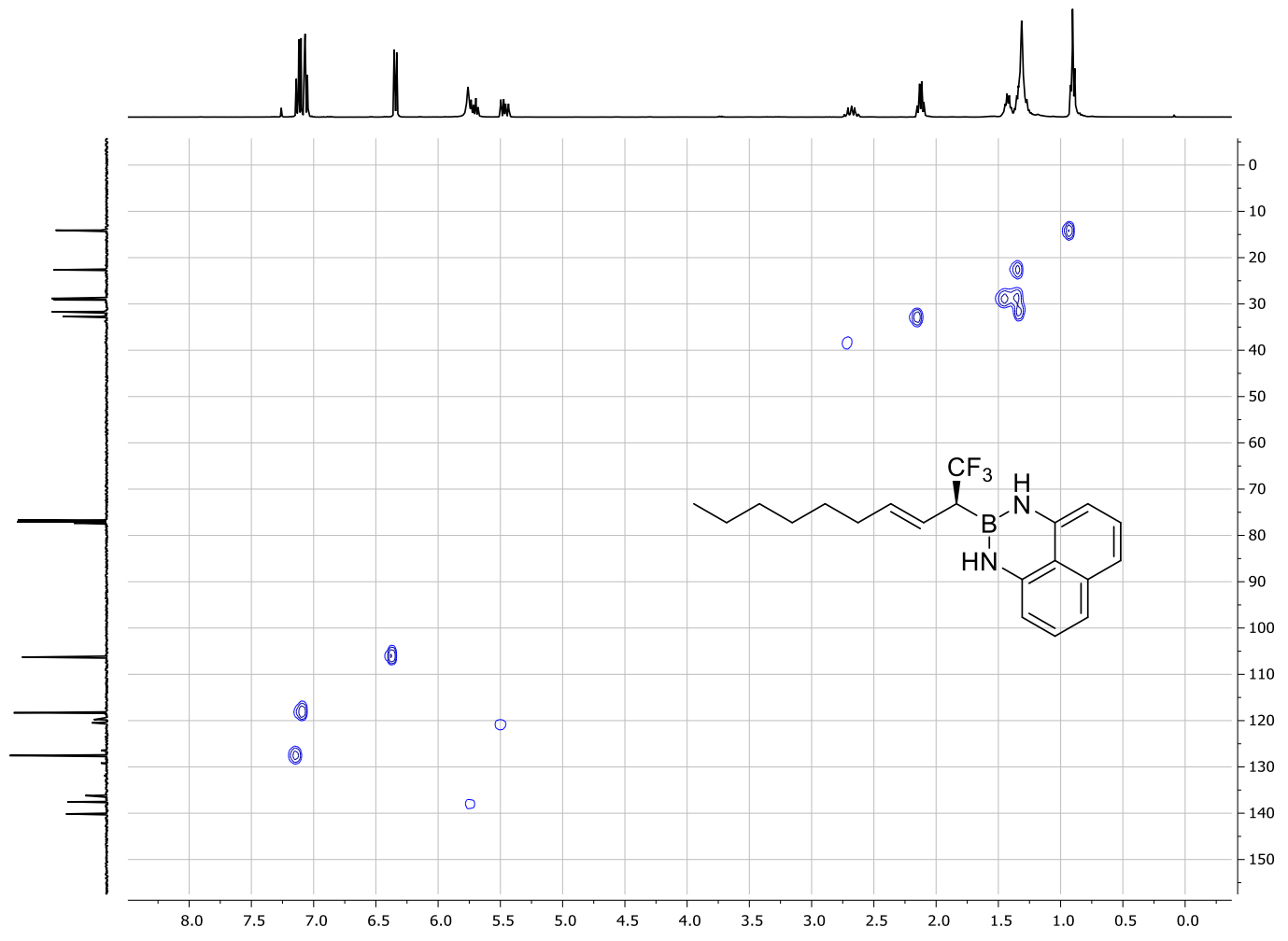

${ }^{19} \mathrm{~F} \mathrm{NMR}\left(\mathrm{CDCl}_{3}, 377 \mathrm{MHz}\right)$ of compound $\mathbf{5 a}$

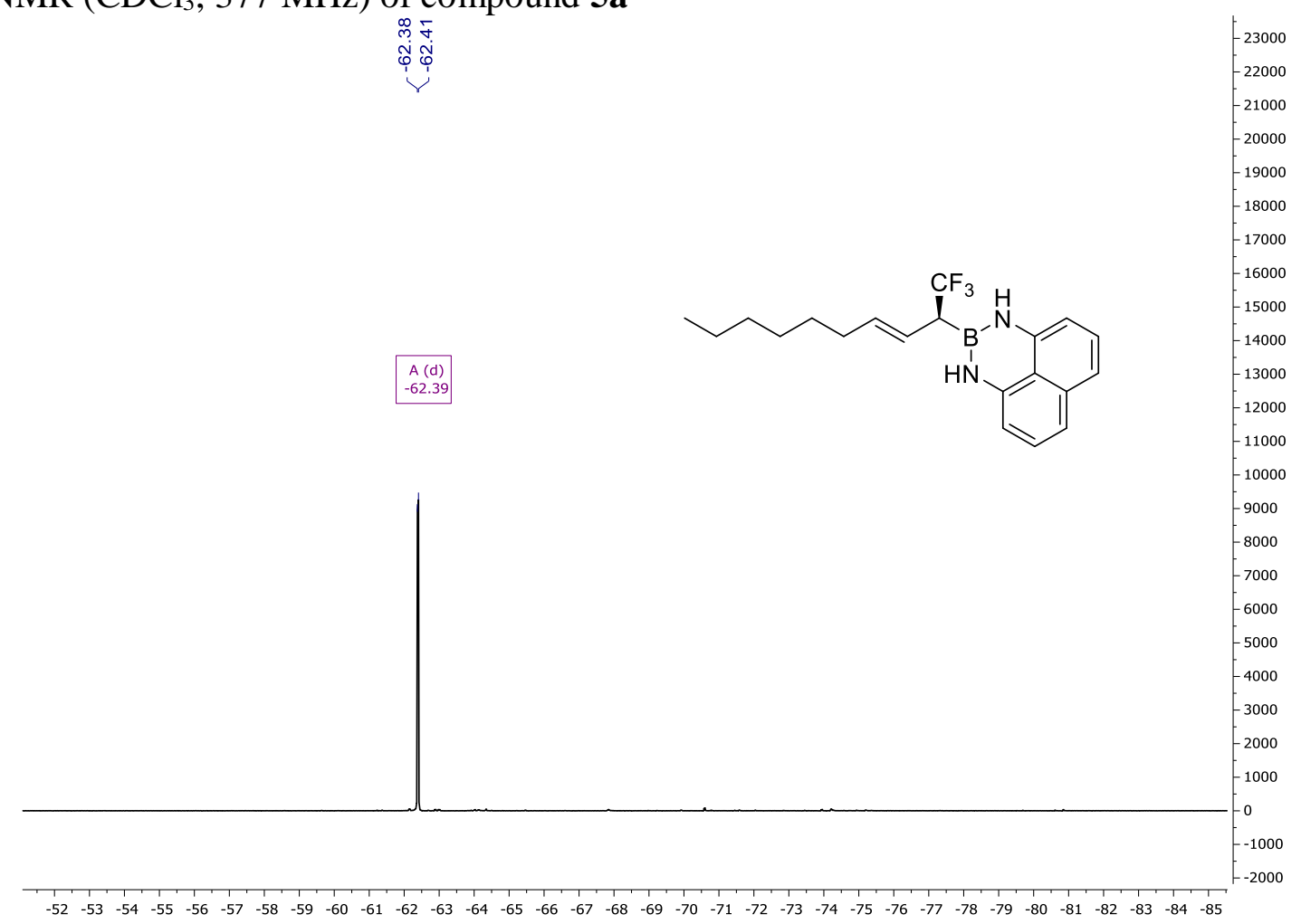


${ }^{11} \mathrm{~B} \mathrm{NMR}\left(\mathrm{CDCl}_{3}, 128 \mathrm{MHz}\right)$ of compound $\mathbf{5 a}$

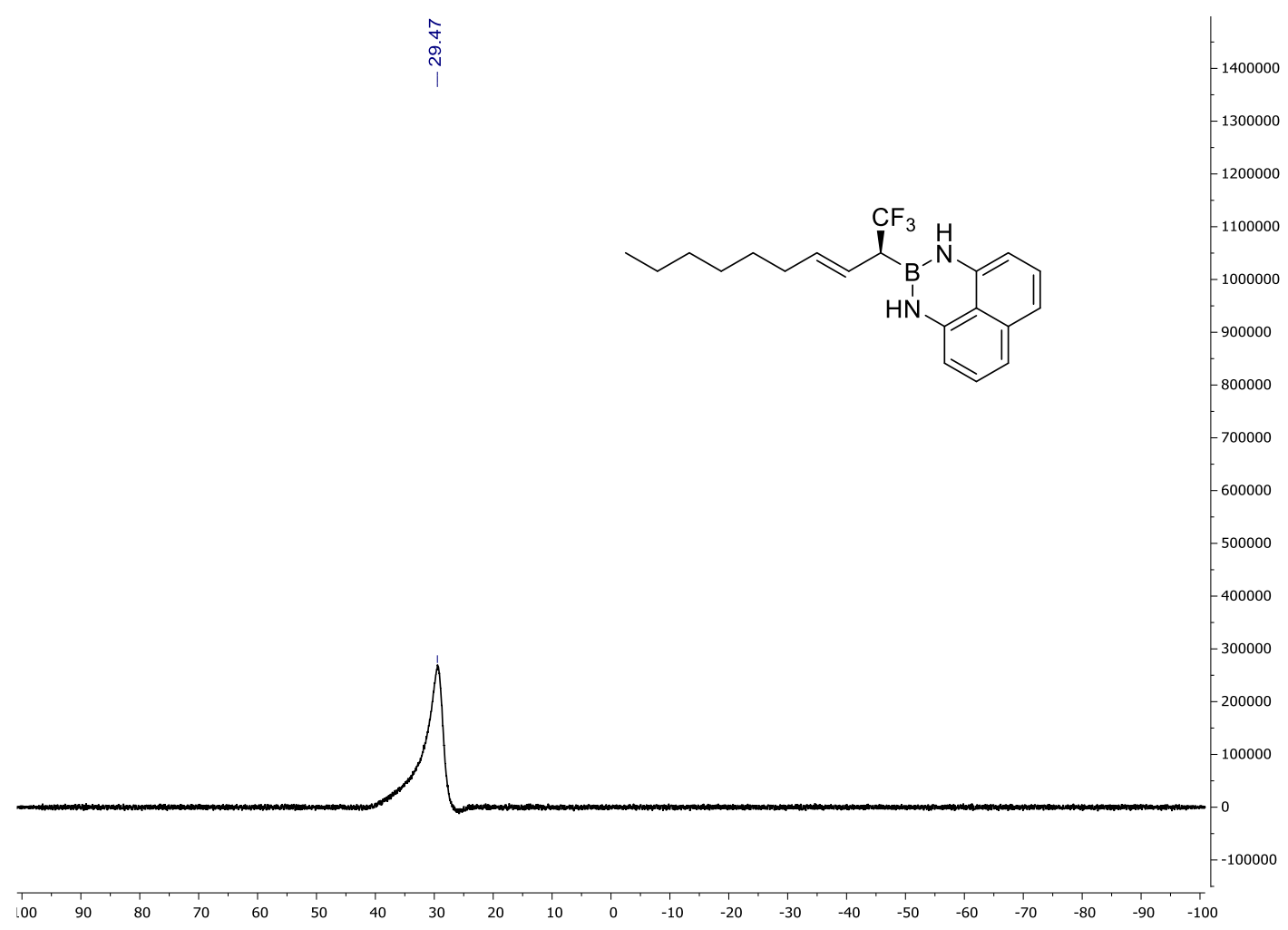

${ }^{1} \mathrm{H} \mathrm{NMR}\left(\mathrm{CDCl}_{3}, 400 \mathrm{MHz}\right)$ of compound $\mathbf{5 b}$

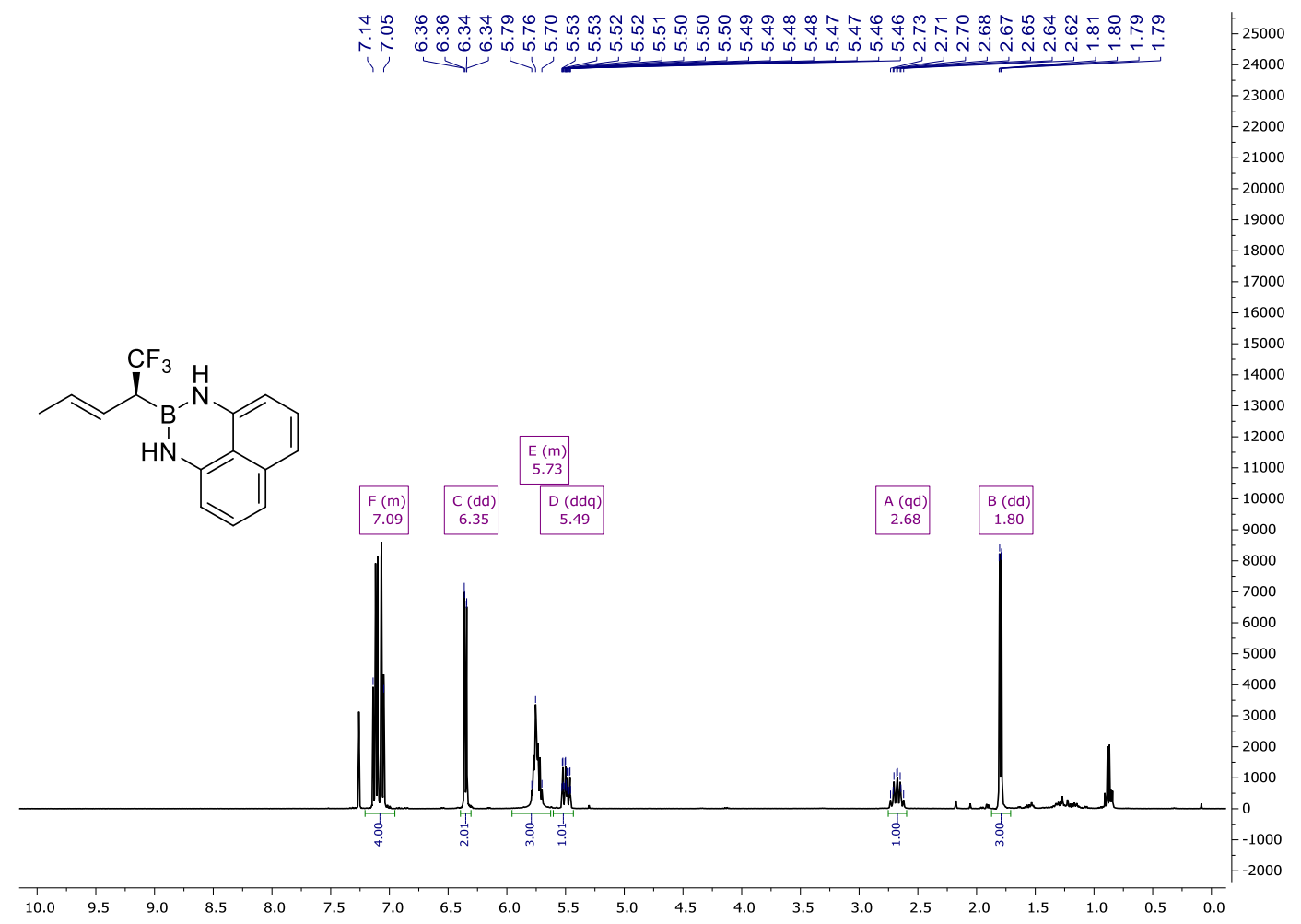


${ }^{13} \mathrm{C}$ NMR $\left(\mathrm{CDCl}_{3}, 101 \mathrm{MHz}\right)$ of compound $\mathbf{5 b}$

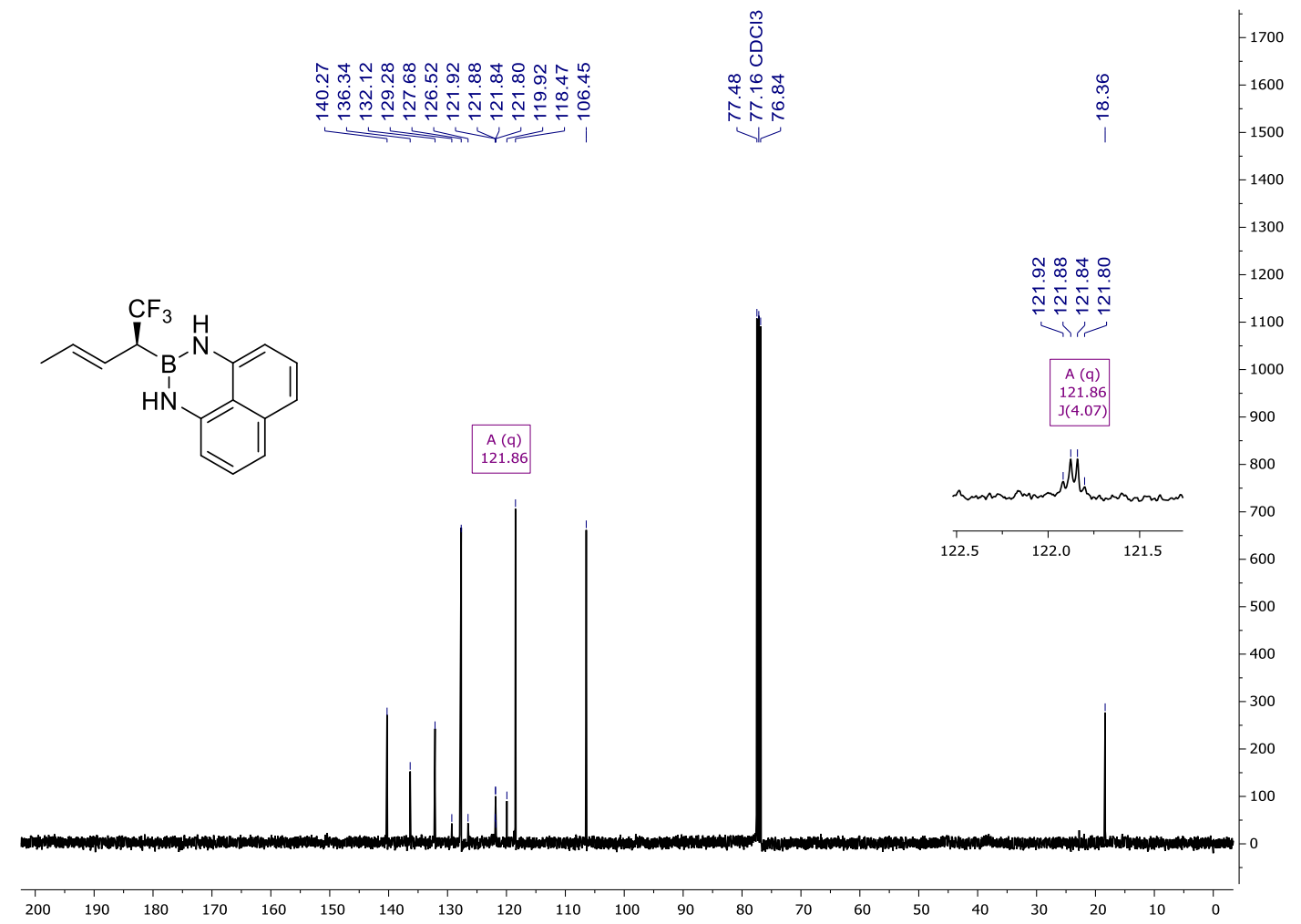

${ }^{19} \mathrm{~F} \mathrm{NMR}\left(\mathrm{CDCl}_{3}, 377 \mathrm{MHz}\right)$ of compound $\mathbf{5 b}$

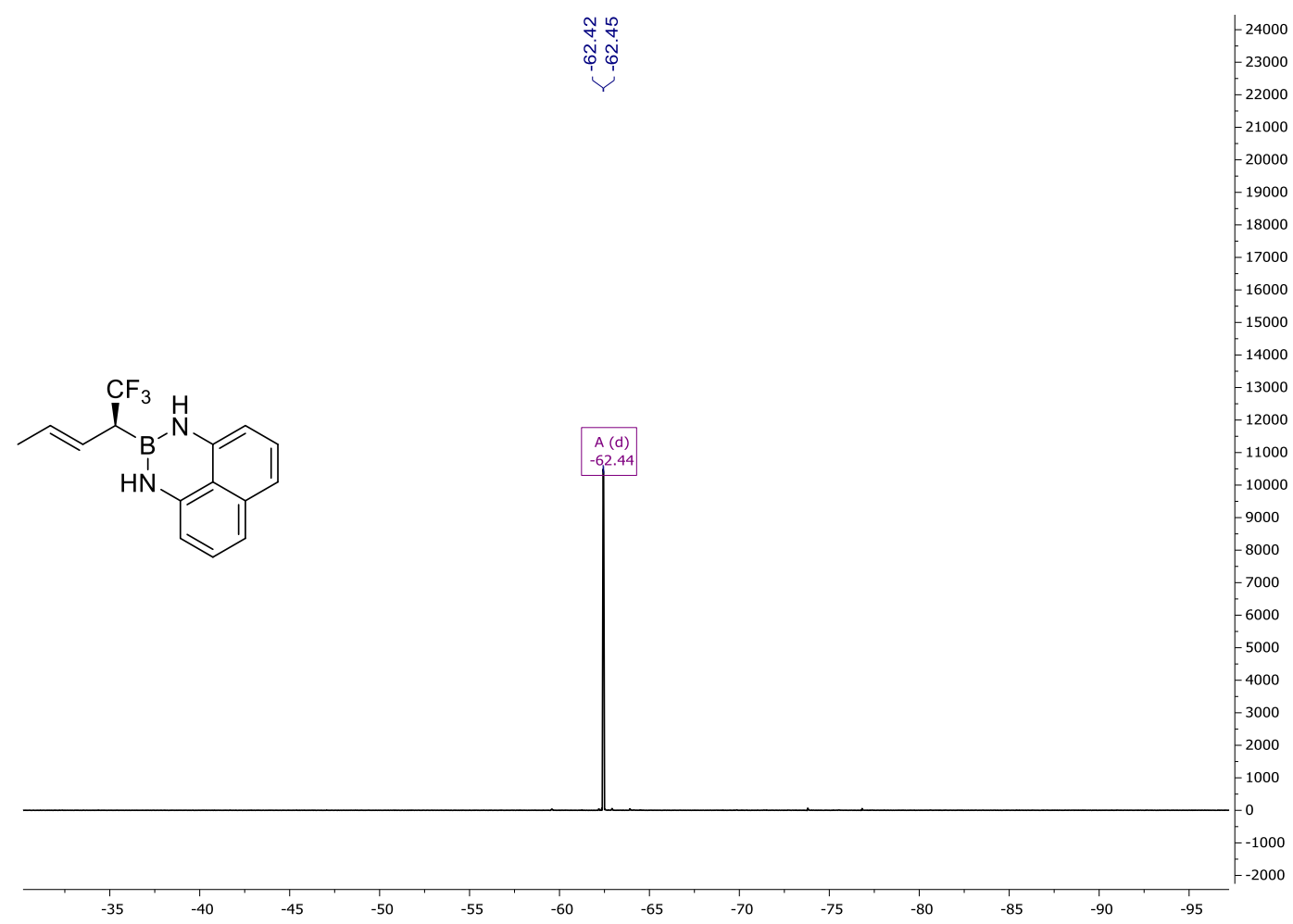


${ }^{11} \mathrm{~B} \mathrm{NMR}\left(\mathrm{CDCl}_{3}, 160 \mathrm{MHz}\right)$ of compound $\mathbf{5 b}$

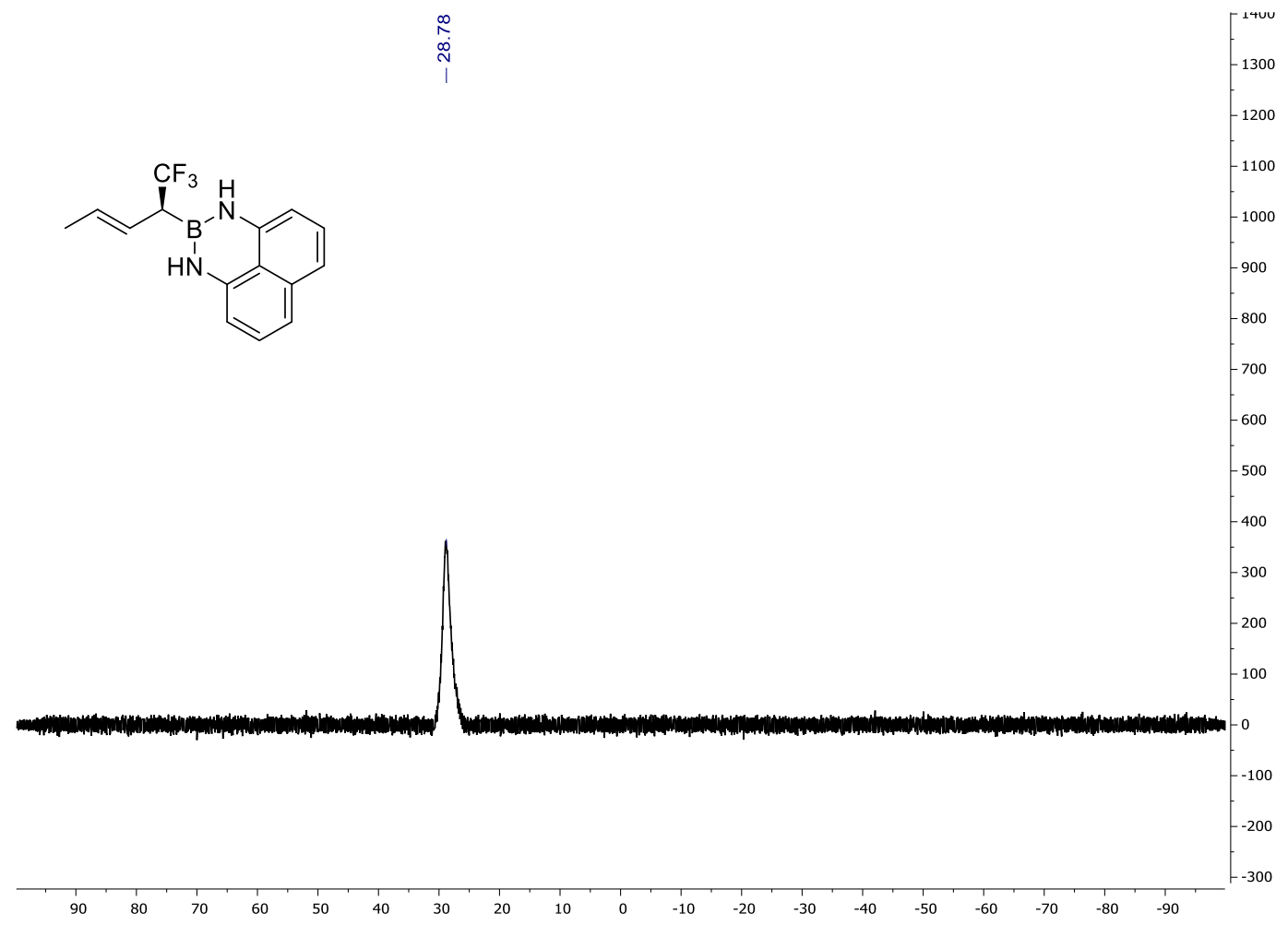

${ }^{1} \mathrm{H} \mathrm{NMR}\left(\mathrm{CDCl}_{3}, 400 \mathrm{MHz}\right)$ of compound $\mathbf{5 c}$

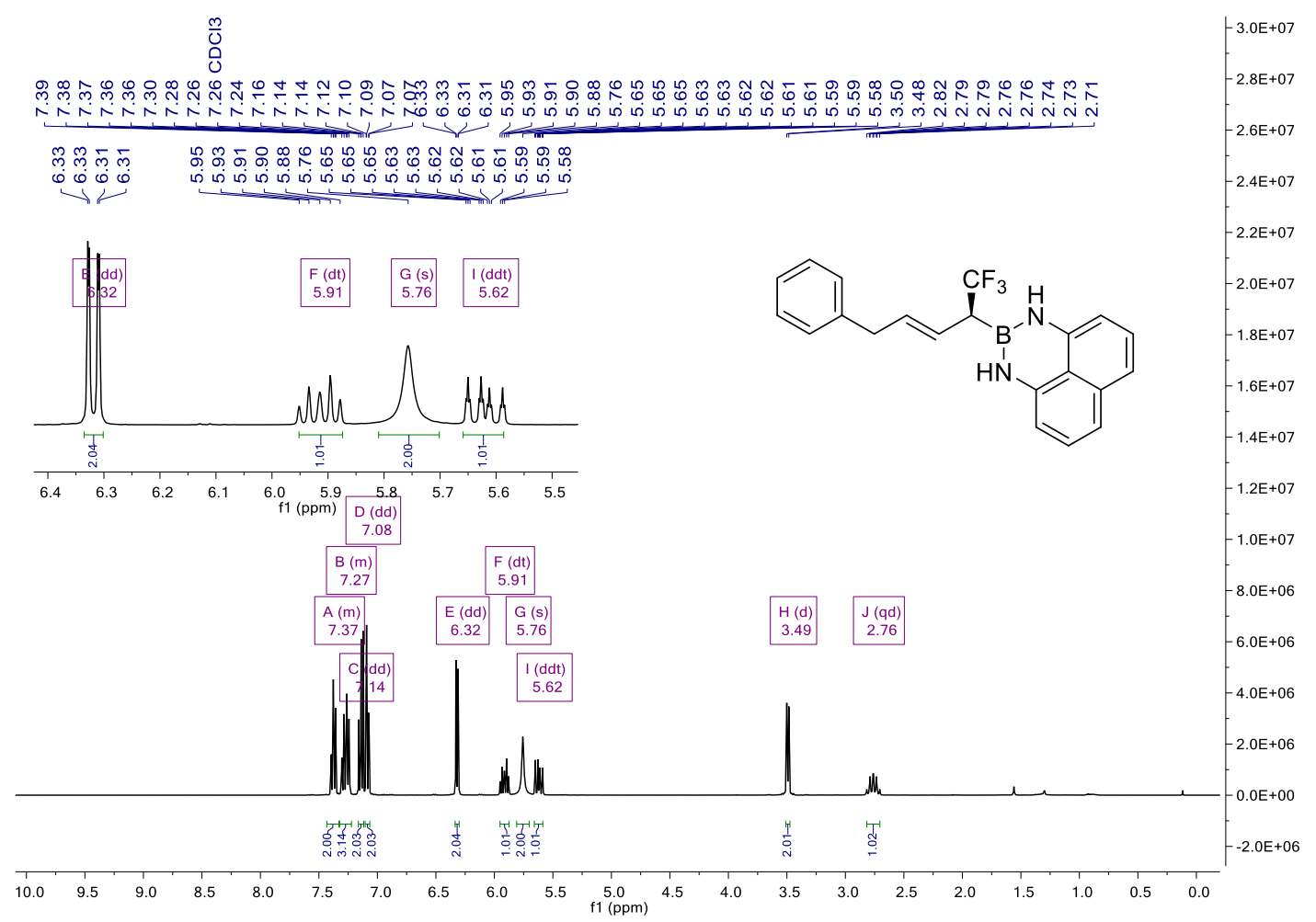


${ }^{13} \mathrm{C} \mathrm{NMR}\left(\mathrm{CDCl}_{3}, 101 \mathrm{MHz}\right)$ of compound $\mathbf{5 c}$

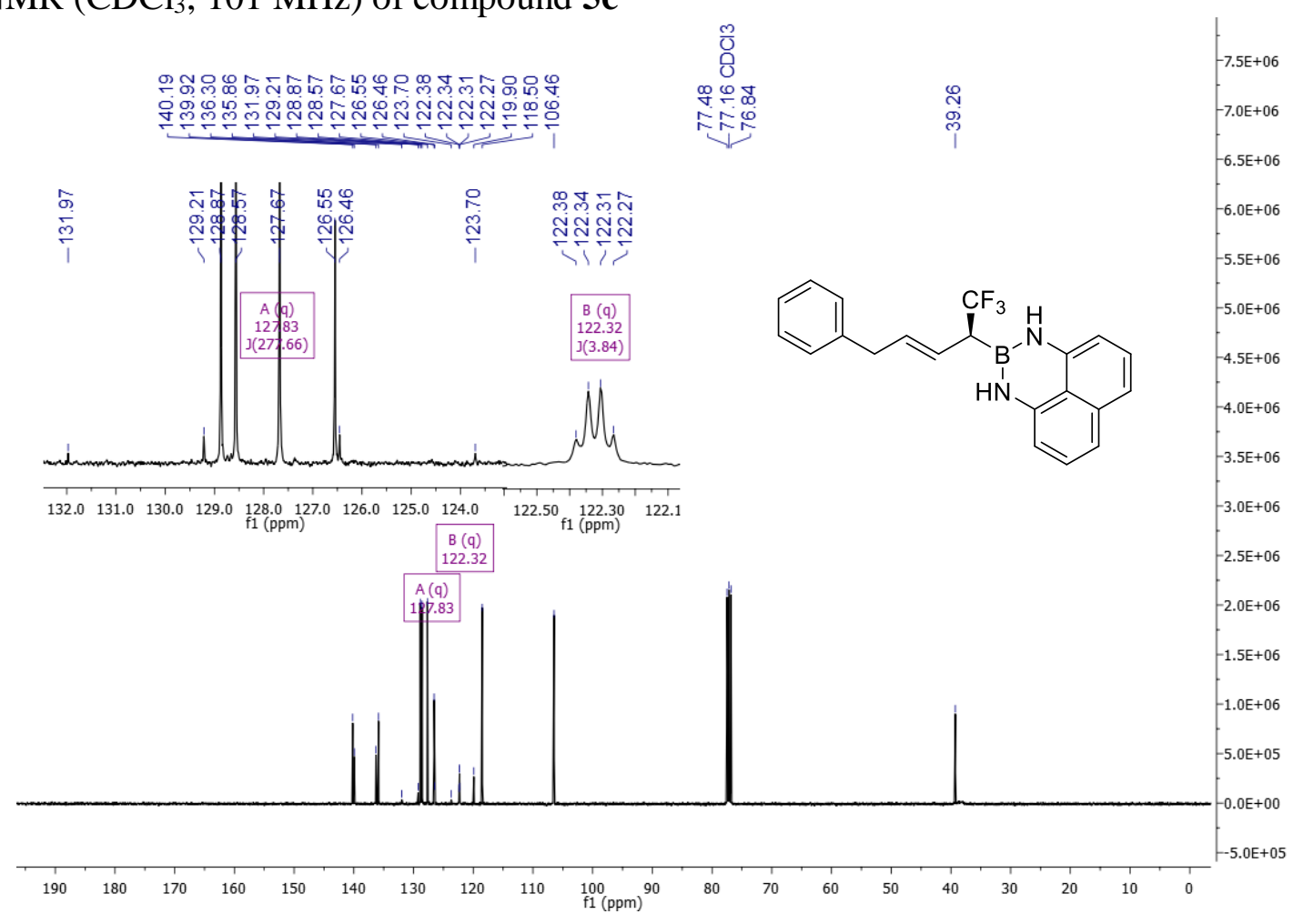

${ }^{19} \mathrm{~F} \mathrm{NMR}\left(\mathrm{CDCl}_{3}, 377 \mathrm{MHz}\right)$ of compound $\mathbf{5 c}$

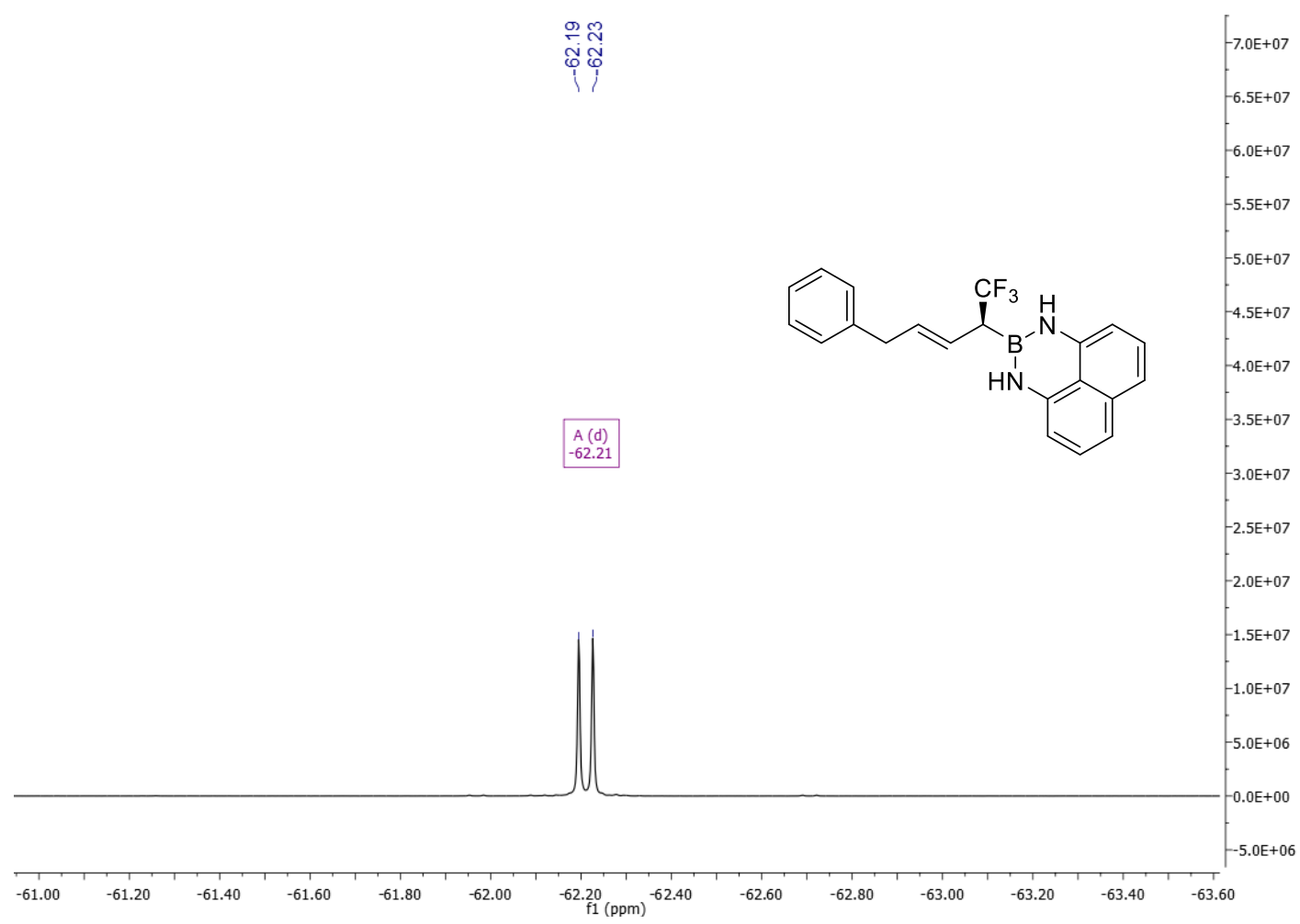


${ }^{11} \mathrm{~B}$ NMR $\left(\mathrm{CDCl}_{3}, 128 \mathrm{MHz}\right)$ of compound $\mathbf{5 c}$

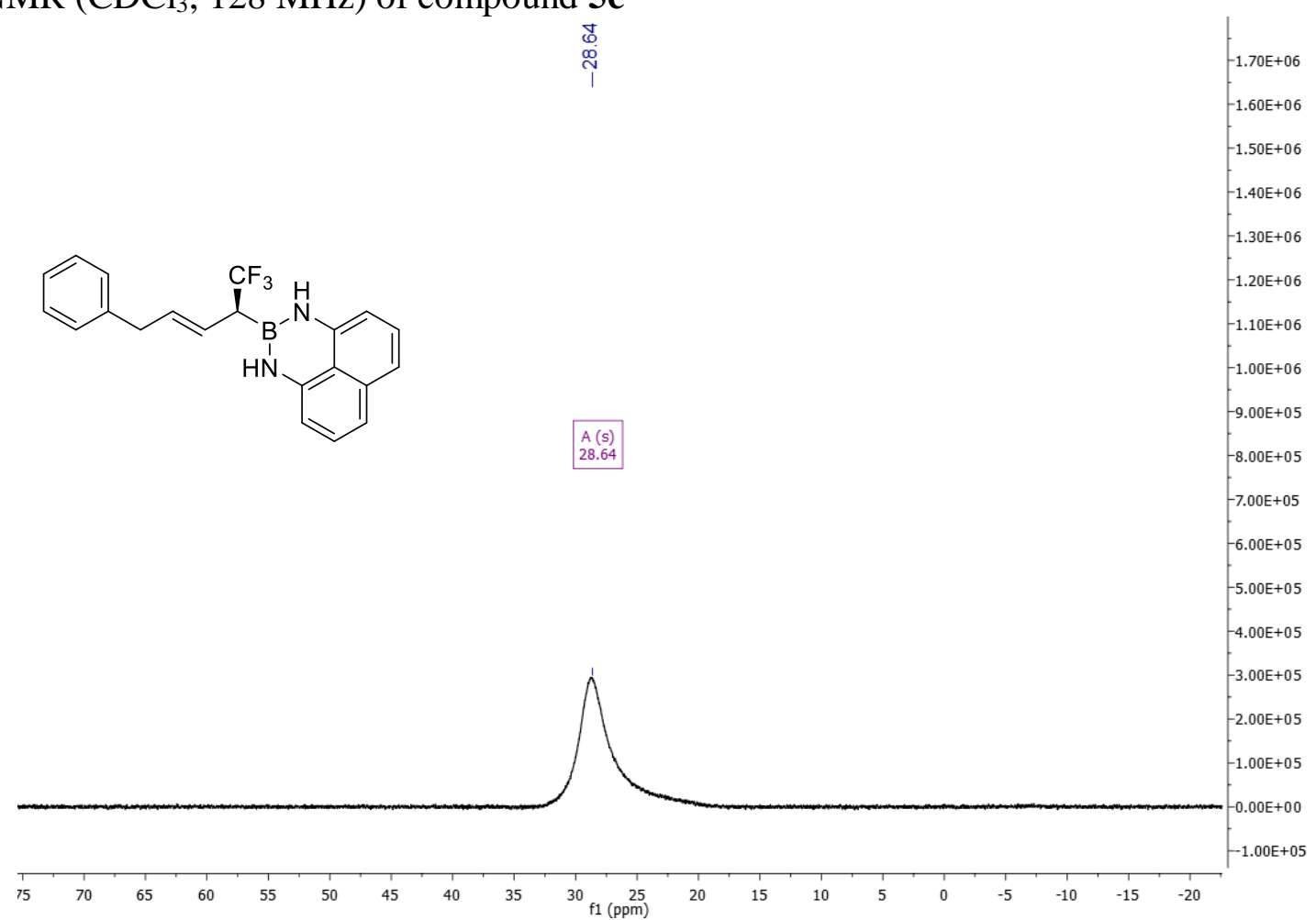

\section{${ }^{1} \mathrm{H} \mathrm{NMR}\left(\mathrm{CDCl}_{3}, 400 \mathrm{MHz}\right)$ of compound $\mathbf{5 d}$}

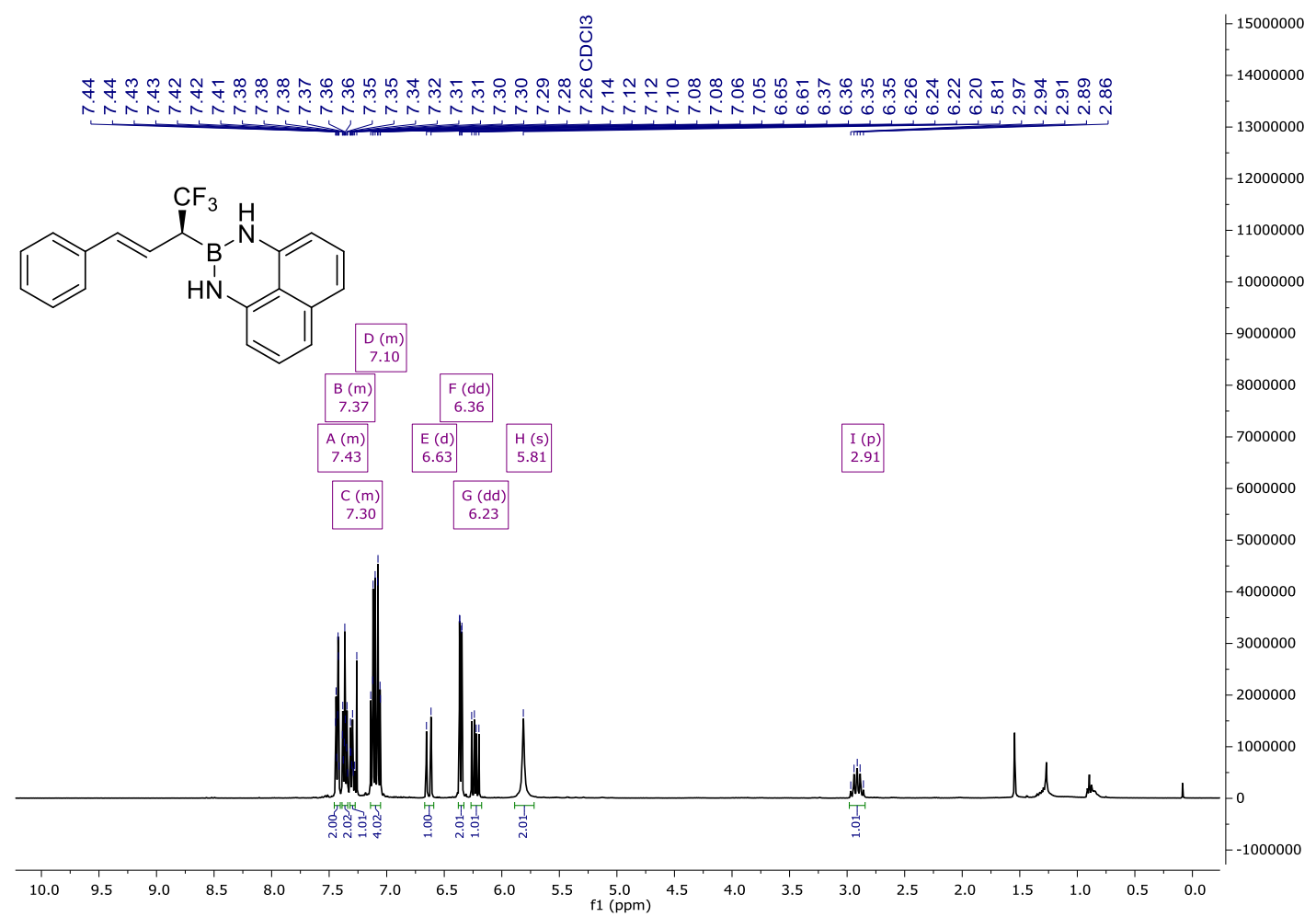


${ }^{13} \mathrm{C} \mathrm{NMR}\left(\mathrm{CDCl}_{3}, 101 \mathrm{MHz}\right)$ of compound $\mathbf{5 d}$
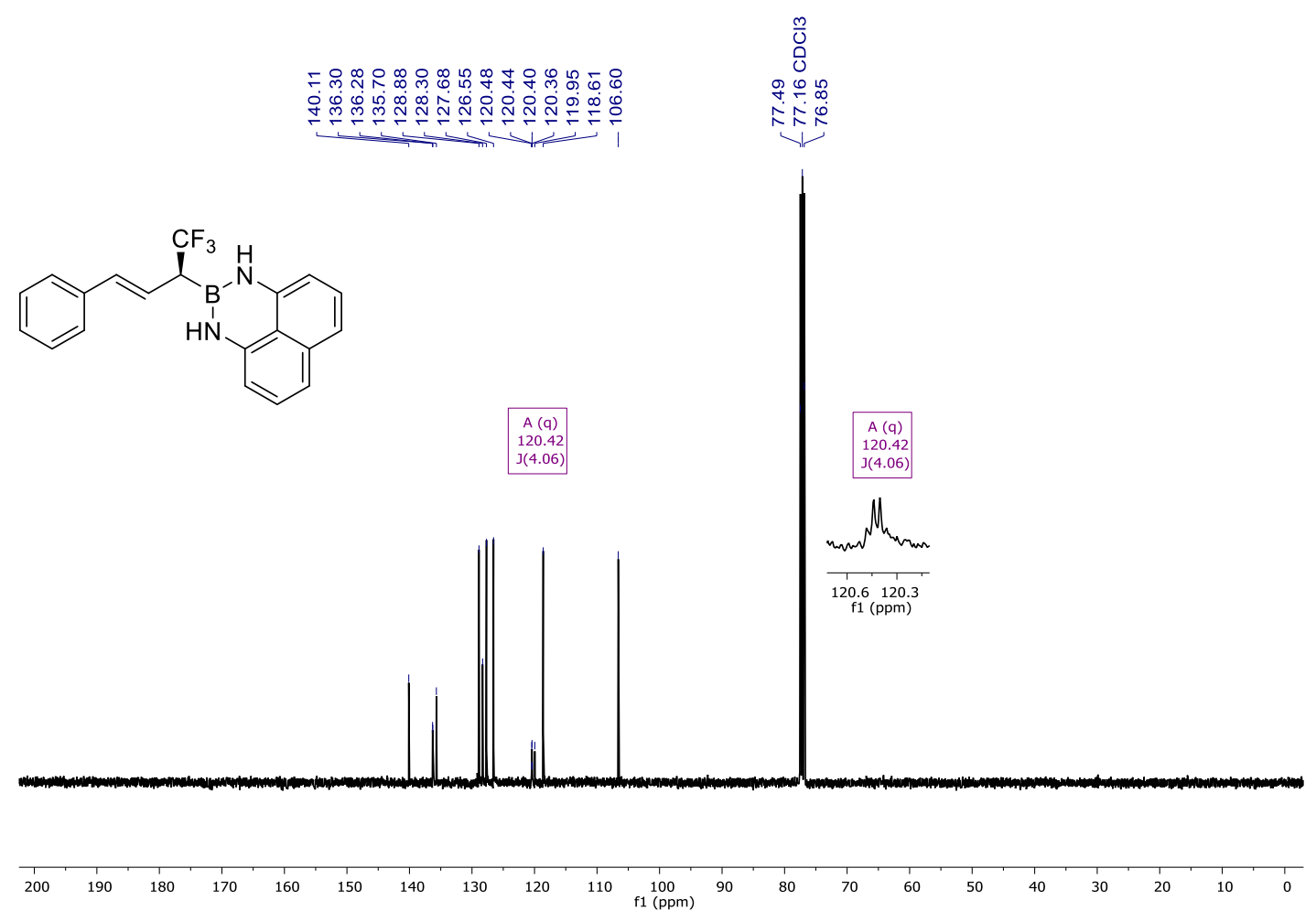

${ }^{19} \mathrm{~F} \mathrm{NMR}\left(\mathrm{CDCl}_{3}, 377 \mathrm{MHz}\right)$ of compound $\mathbf{5 d}$

กู

फ़ڤ<smiles>FC(F)(F)[C@H](/C=C/c1ccccc1)B1Nc2cccc3cccc(c23)N1</smiles>

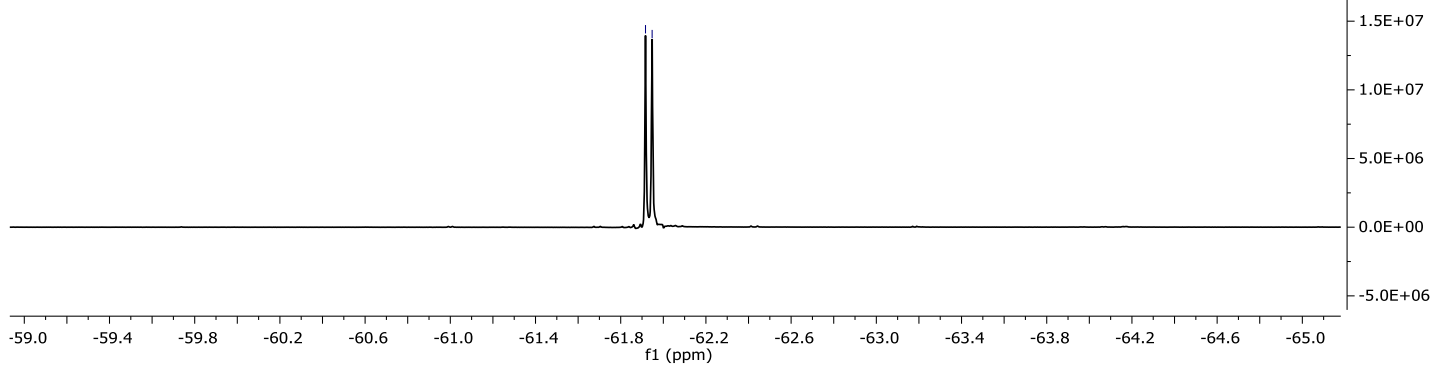


${ }^{11} \mathrm{~B}$ NMR $\left(\mathrm{CDCl}_{3}, 128 \mathrm{MHz}\right)$ of compound $\mathbf{5 d}$

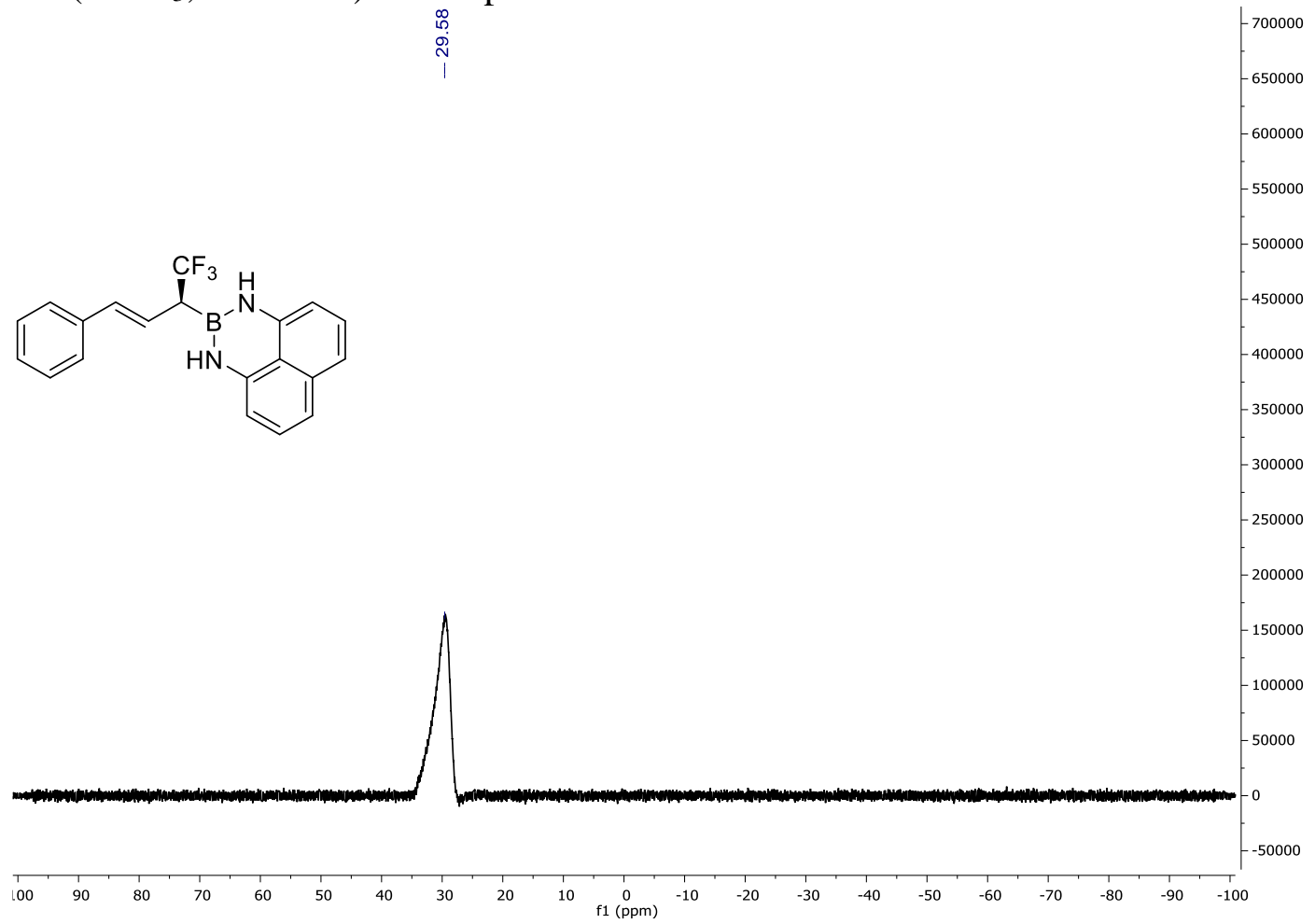

${ }^{1} \mathrm{H} \mathrm{NMR}\left(\mathrm{CDCl}_{3}, 400 \mathrm{MHz}\right)$ of compound $\mathbf{5 e}$

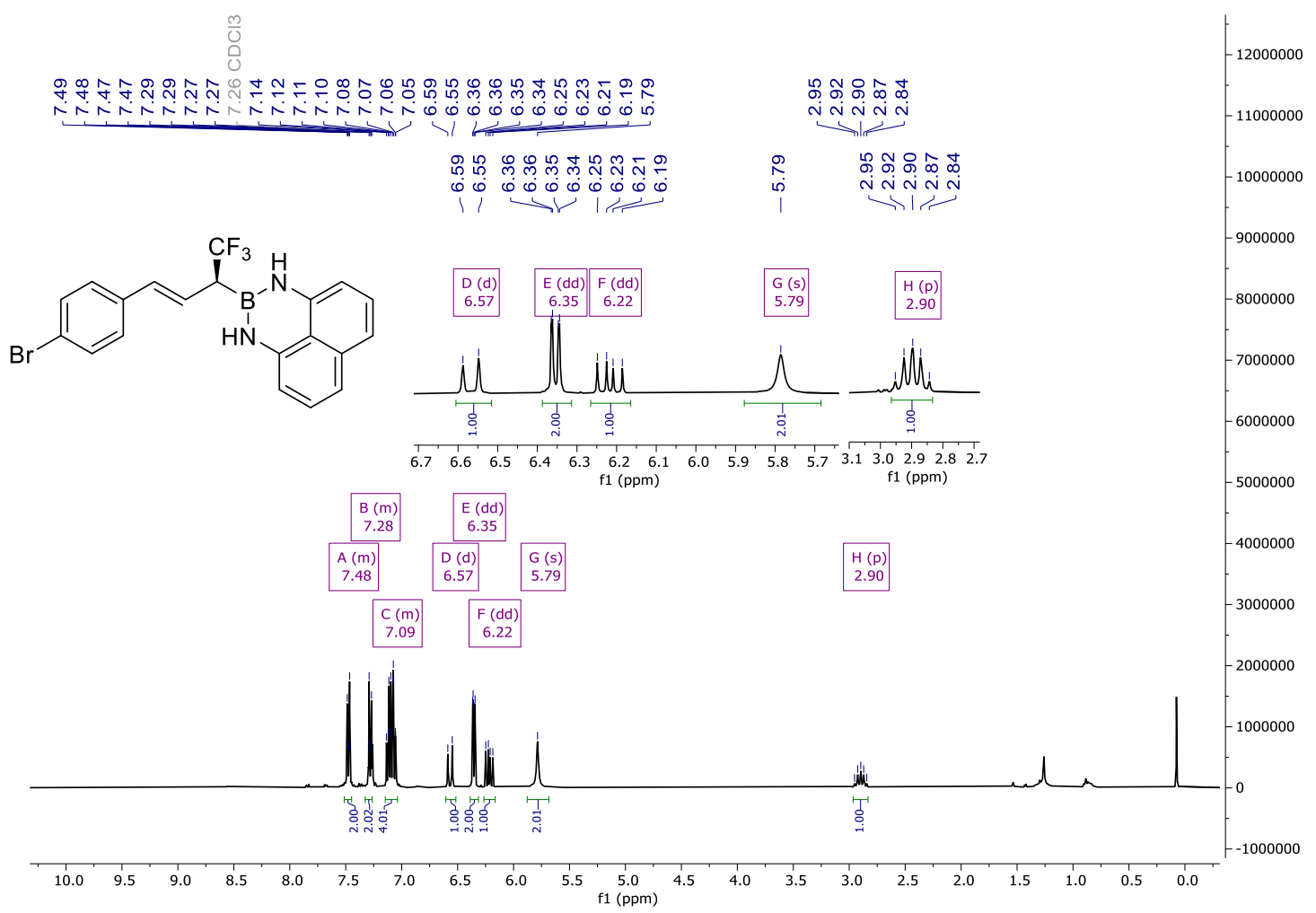


${ }^{13} \mathrm{C}$ NMR $\left(\mathrm{CDCl}_{3}, 126 \mathrm{MHz}\right)$ of compound $\mathbf{5 e}$

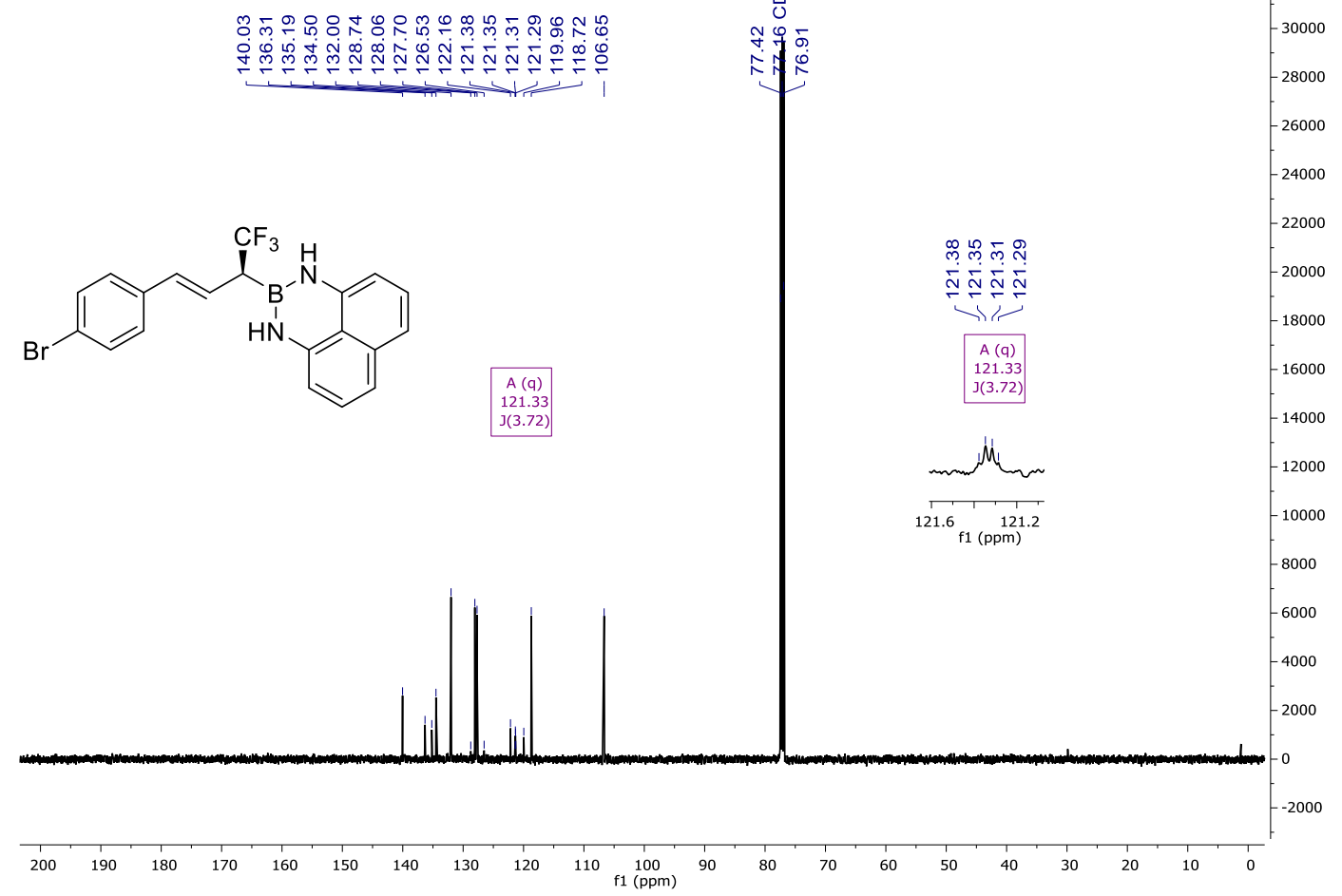

${ }^{19} \mathrm{~F} \mathrm{NMR}\left(\mathrm{CDCl}_{3}, 377 \mathrm{MHz}\right)$ of compound $\mathbf{5 e}$<smiles>FC(F)(F)C(/C=C/c1ccc(Br)cc1)B1Nc2cccc3cccc(c23)N1</smiles>

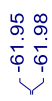

\begin{tabular}{llllllllllllllllllllllll}
\hline & -53 & -54 & -55 & -56 & -57 & -58 & -59 & -60 & -61 & -62 & -63 & -64 & -65 & -66 & -67 & -68 & -69 & -70 & -71 & -72 & -73
\end{tabular} 
${ }^{11} \mathrm{~B}$ NMR $\left(\mathrm{CDCl}_{3}, 160 \mathrm{MHz}\right)$ of compound $\mathbf{5 e}$

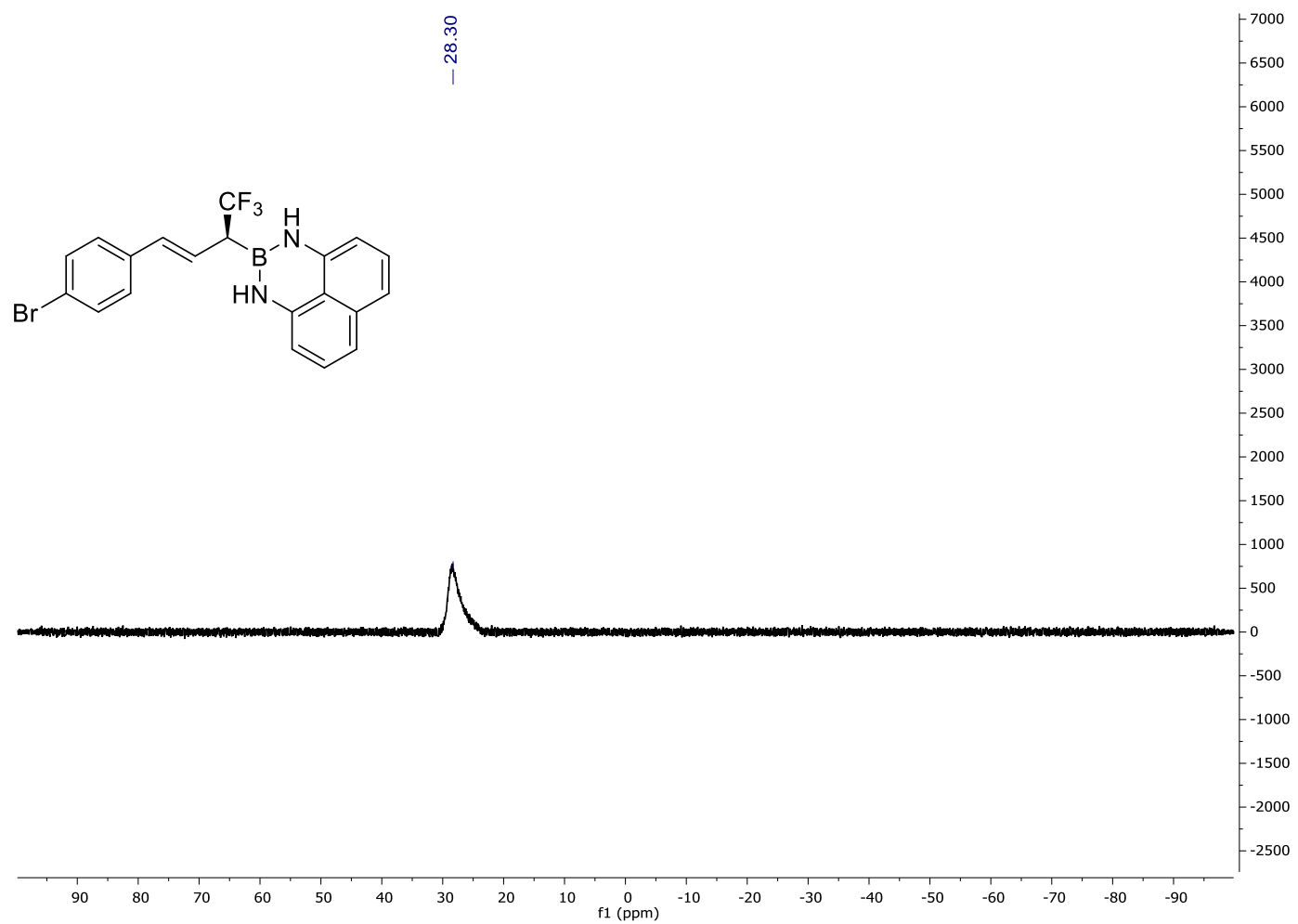

${ }^{1} \mathrm{H} \mathrm{NMR}\left(\mathrm{CDCl}_{3}, 400 \mathrm{MHz}\right)$ of compound $\mathbf{5 f}$

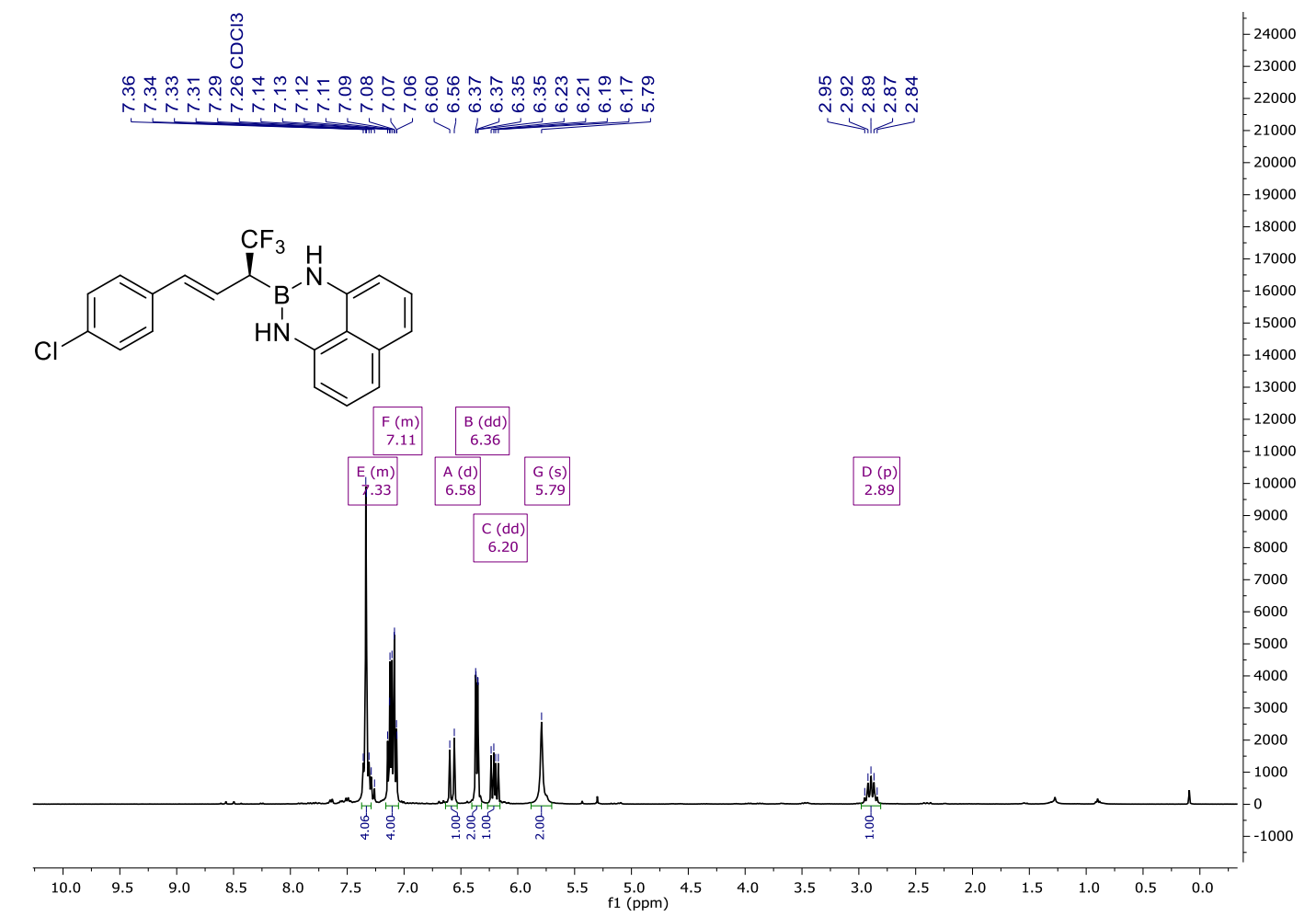


${ }^{13} \mathrm{C}$ NMR $\left(\mathrm{CDCl}_{3}, 101 \mathrm{MHz}\right)$ of compound $\mathbf{5 f}$

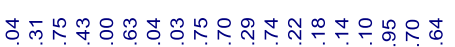

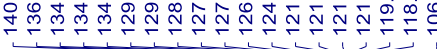

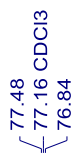

(c)

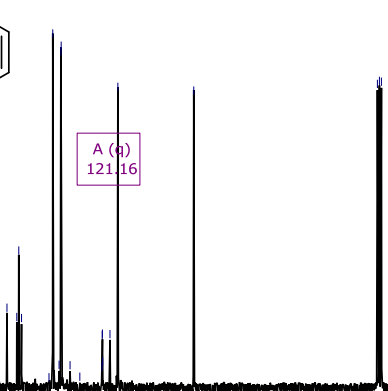

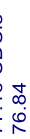

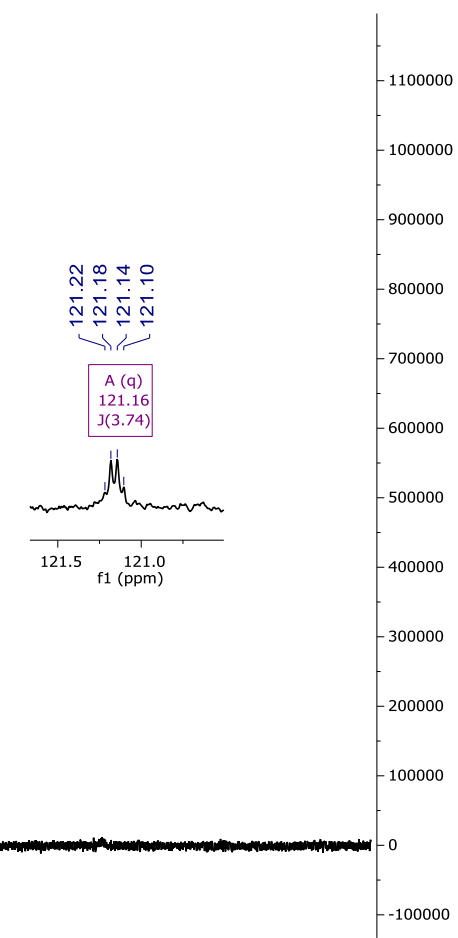

$\begin{array}{lllllllllll}200 & 190 & 180 & 170 & 160 & 150 & 140 & 130 & 120 & 110 & 100\end{array}$

${ }^{19} \mathrm{~F} \mathrm{NMR}\left(\mathrm{CDCl}_{3}, 377 \mathrm{MHz}\right)$ of compound $\mathbf{5 f}$

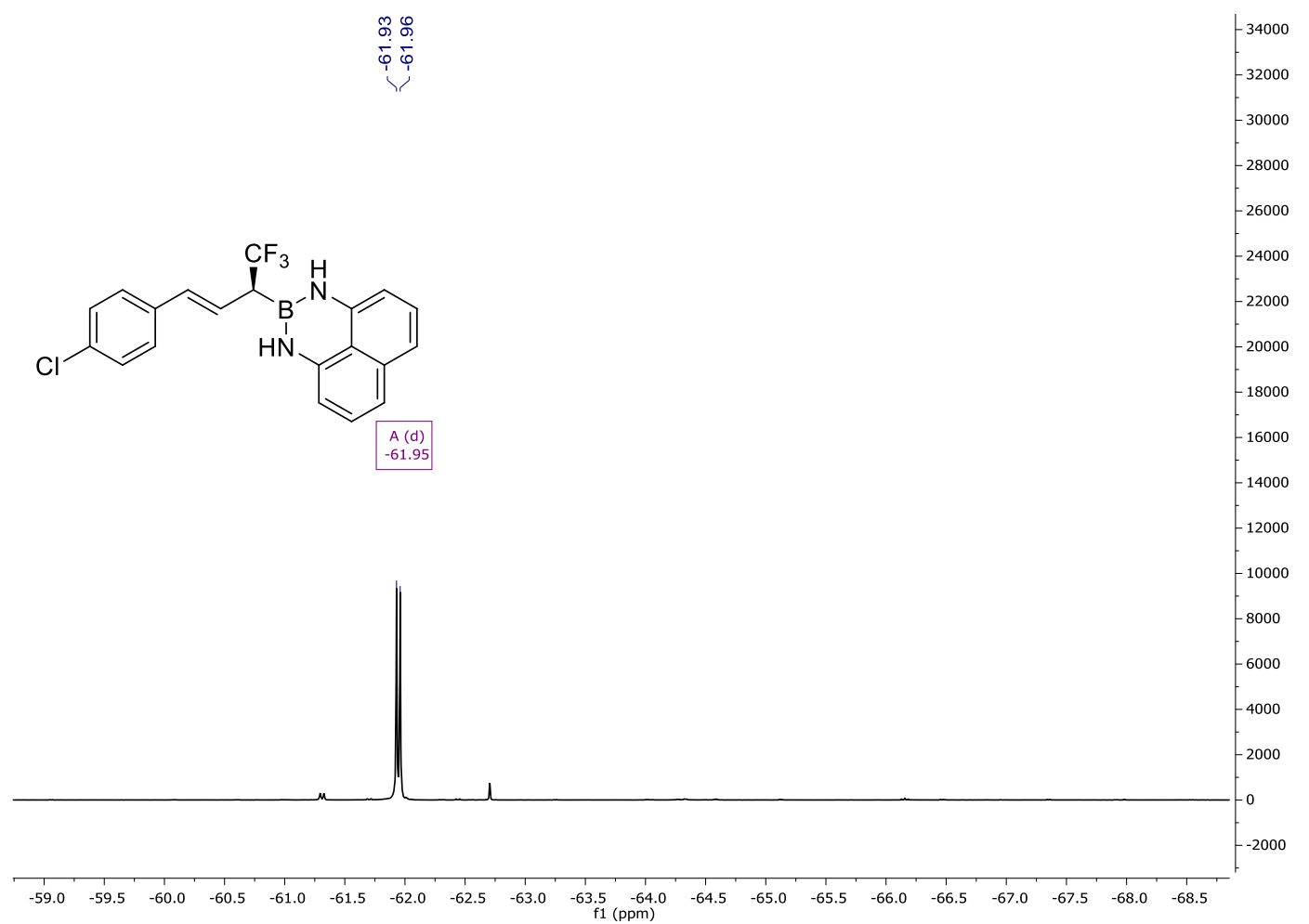


${ }^{11} \mathrm{~B}$ NMR $\left(\mathrm{CDCl}_{3}, 128 \mathrm{MHz}\right)$ of compound $\mathbf{5 e}$

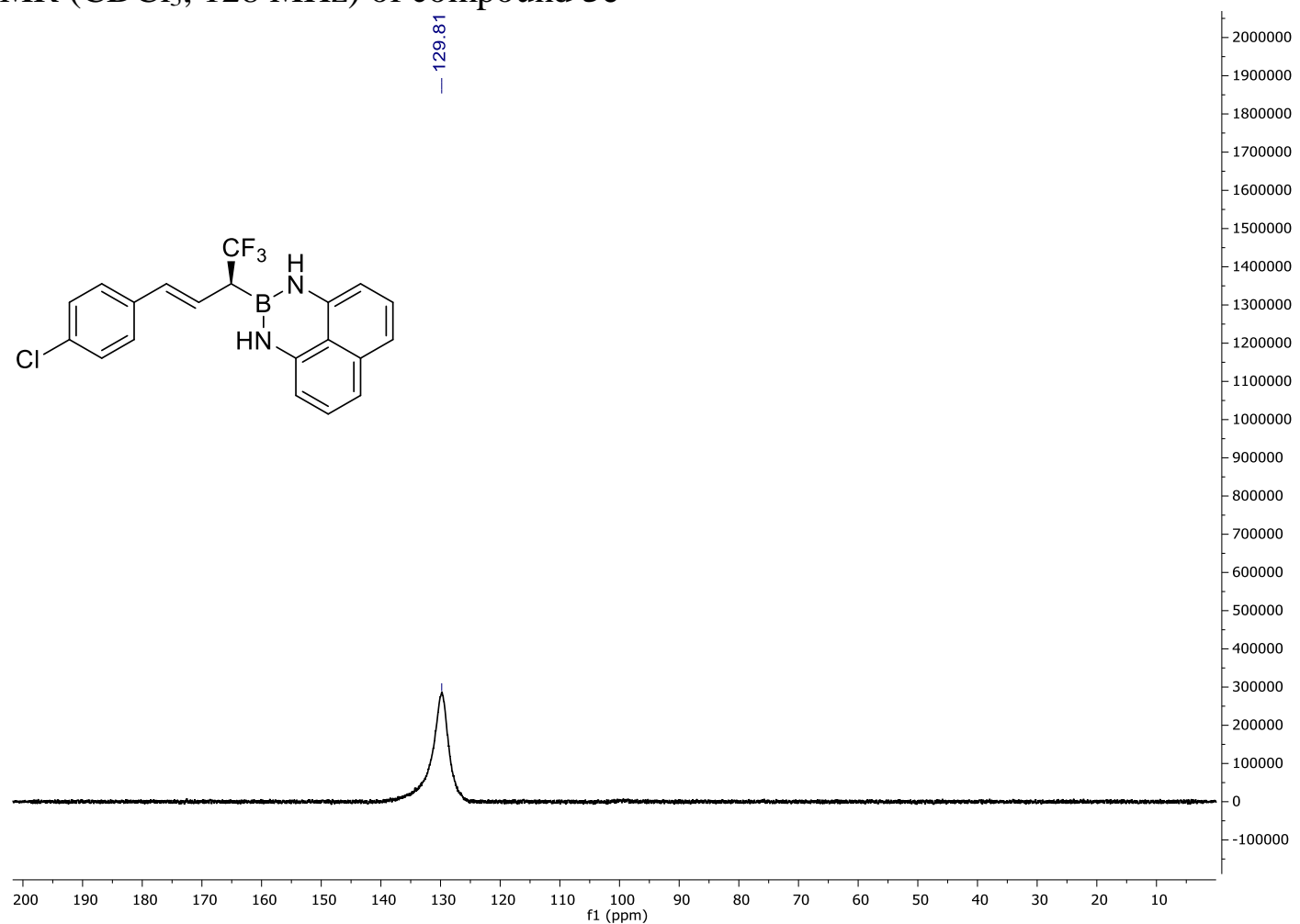

${ }^{1} \mathrm{H} \mathrm{NMR}\left(\mathrm{CDCl}_{3}, 400 \mathrm{MHz}\right)$ of compound $\mathbf{5 g}$

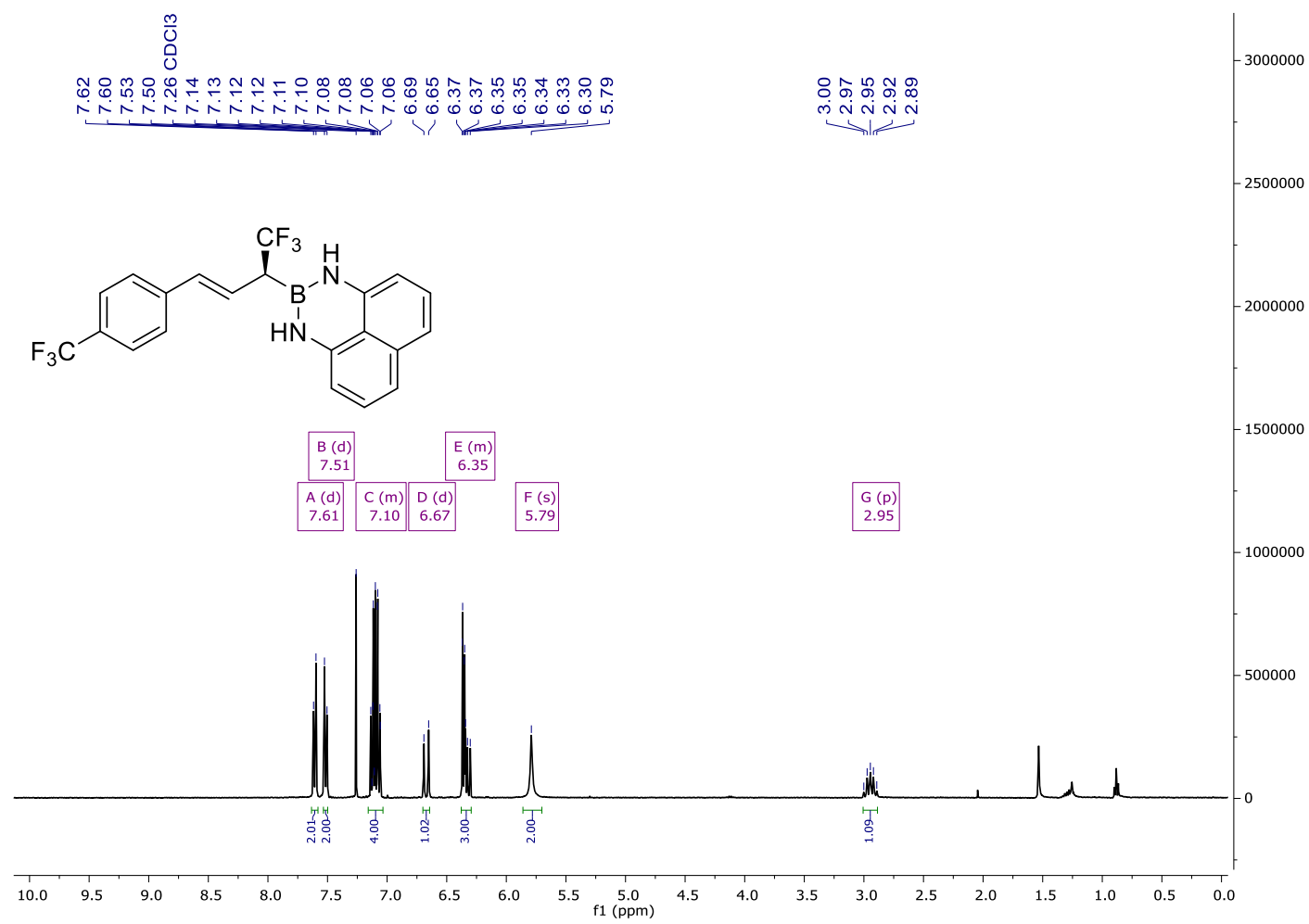


${ }^{13} \mathrm{C}$ NMR $\left(\mathrm{CDCl}_{3}, 101 \mathrm{MHz}\right)$ of compound $\mathbf{5 f}$

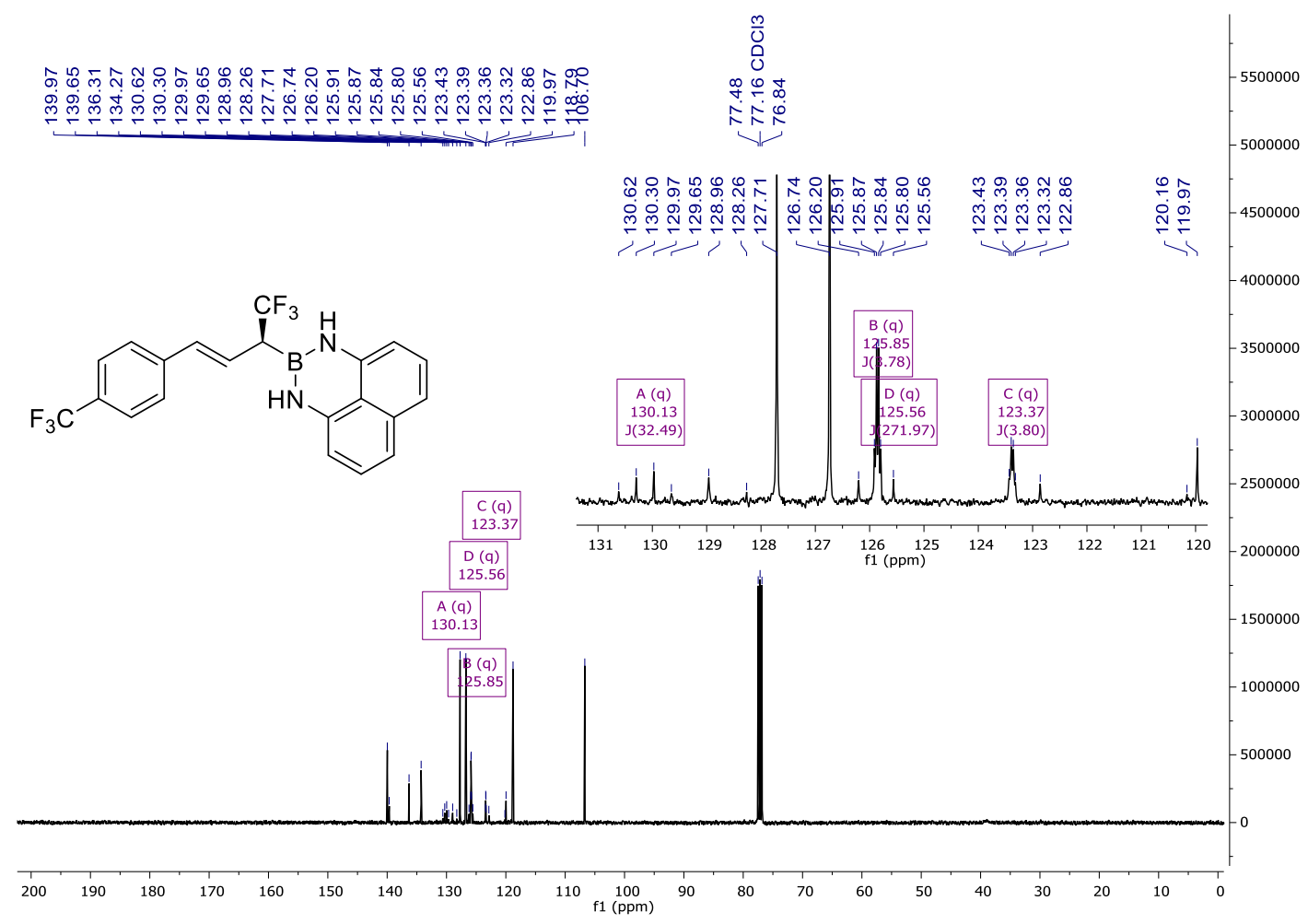

${ }^{19} \mathrm{~F} \mathrm{NMR}\left(\mathrm{CDCl}_{3}, 377 \mathrm{MHz}\right)$ of compound $\mathbf{5 g}$

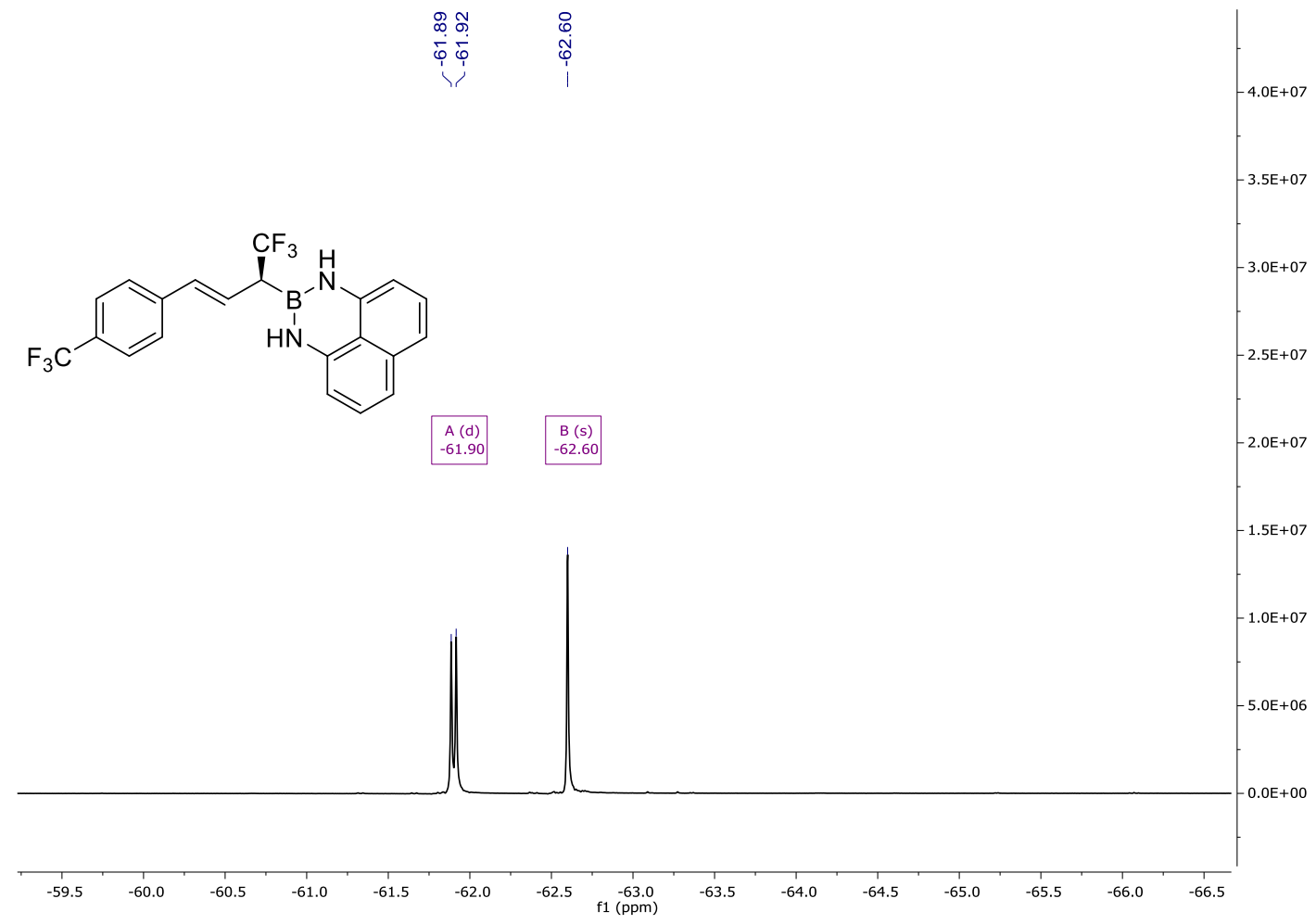


${ }^{11} \mathrm{~B}$ NMR $\left(\mathrm{CDCl}_{3}, 128 \mathrm{MHz}\right)$ of compound $\mathbf{5 g}$

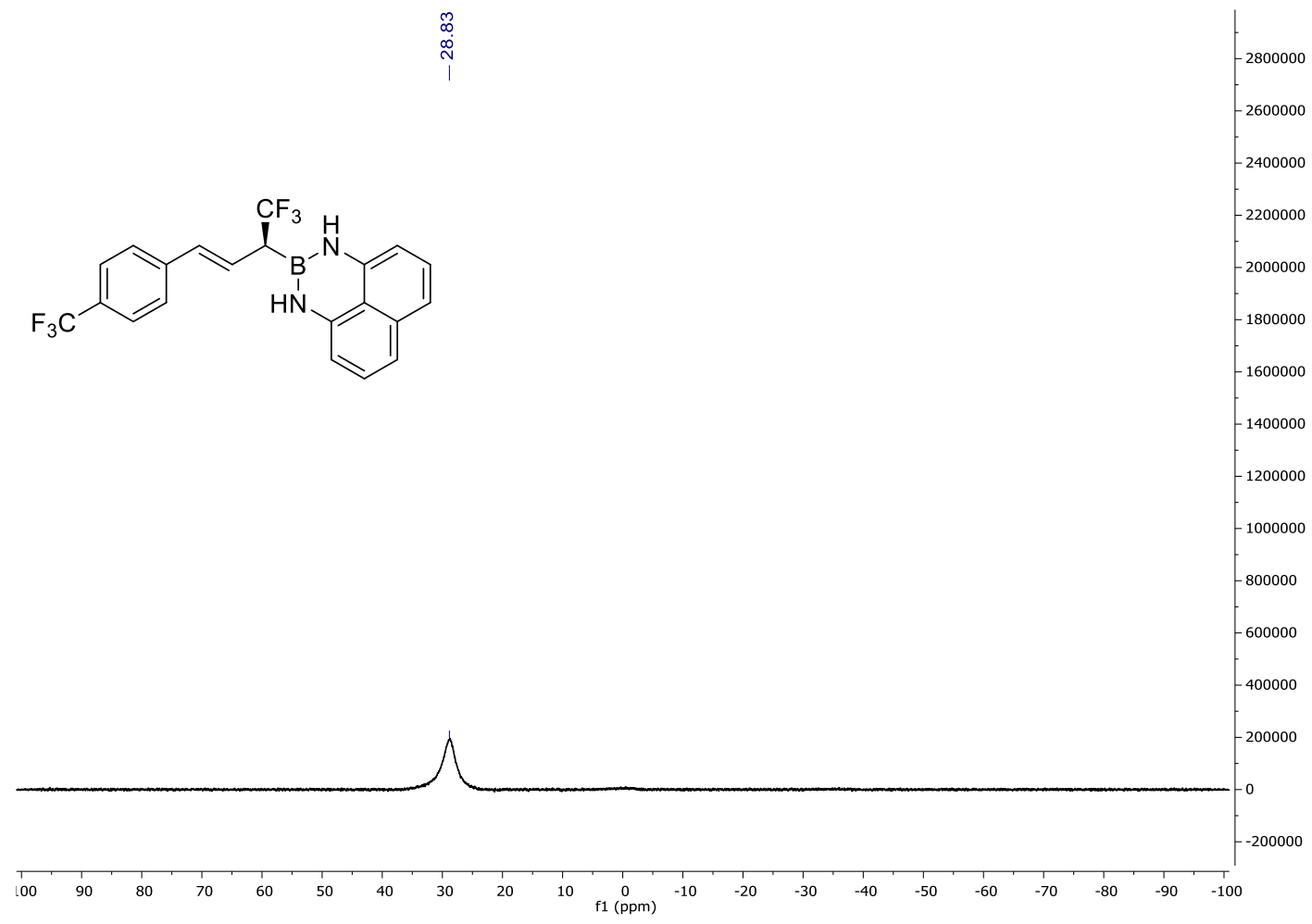

${ }^{1} \mathrm{H} \mathrm{NMR}\left(\mathrm{CDCl}_{3}, 400 \mathrm{MHz}\right)$ of compound $\mathbf{7 a}$

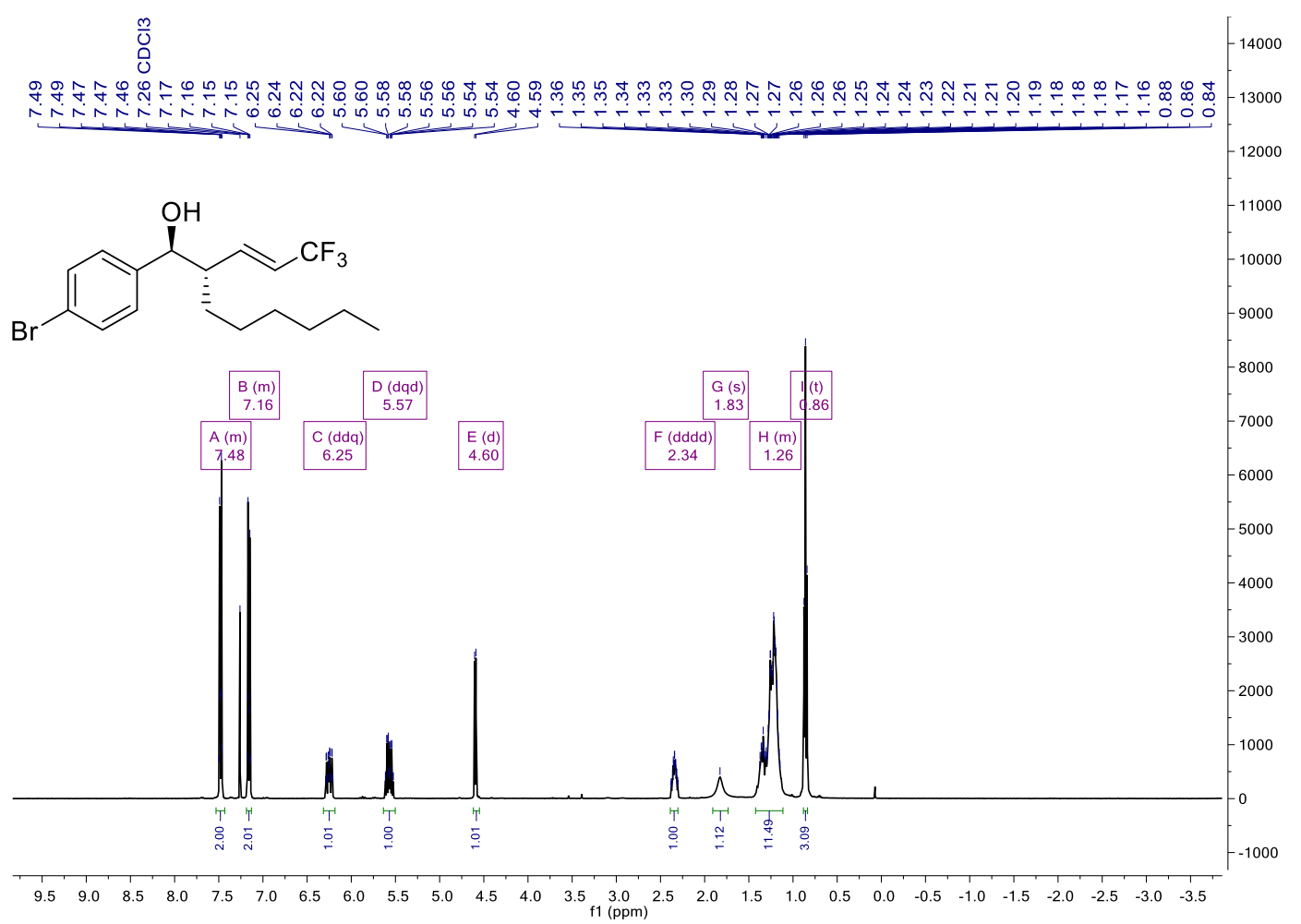


${ }^{13} \mathrm{C} \mathrm{NMR}\left(\mathrm{CDCl}_{3}, 101 \mathrm{MHz}\right)$ of compound $\mathbf{7 a}$

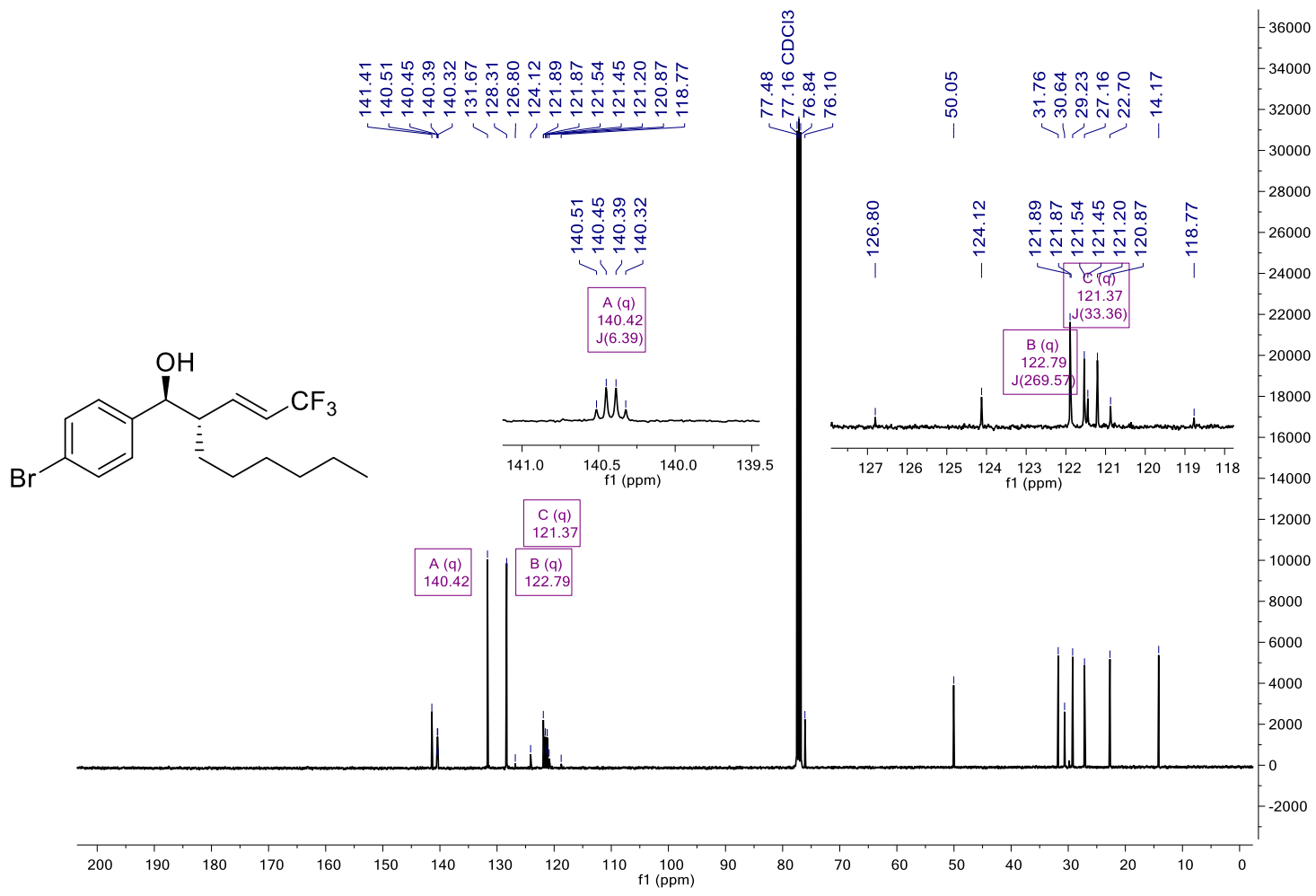

${ }^{19} \mathrm{~F} \mathrm{NMR}\left(\mathrm{CDCl}_{3}, 377 \mathrm{MHz}\right)$ of compound $7 \mathbf{a}$<smiles>CCCCCC[C@H](/C=C/C(F)(F)F)[C@H](O)c1ccc(Br)cc1</smiles>

$\infty$ 용ㅇㅇㅇ

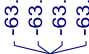

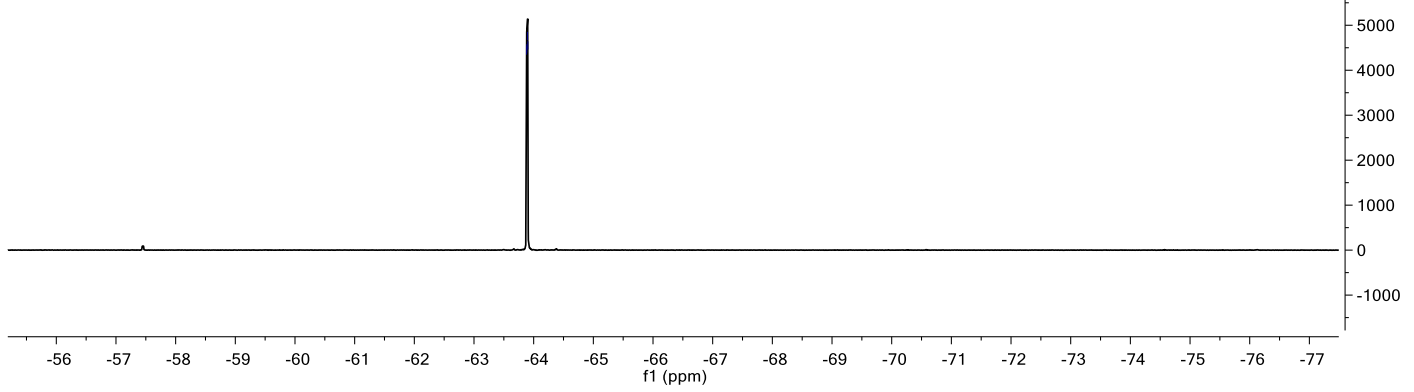


${ }^{1} \mathrm{H}$ NMR $\left(\mathrm{CDCl}_{3}, 400 \mathrm{MHz}\right)$ of compound $\mathbf{7 a}$

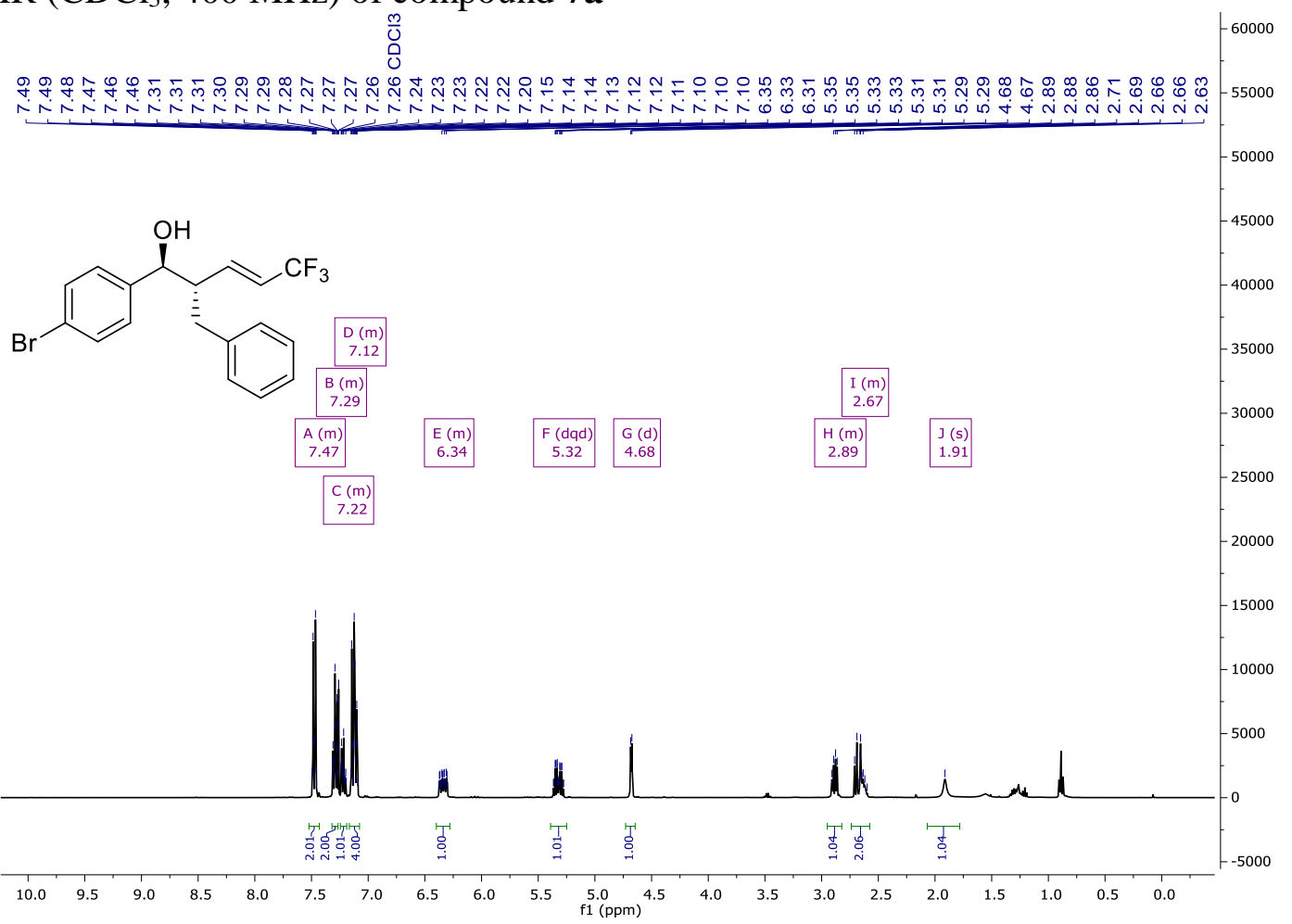

${ }^{13} \mathrm{C} \mathrm{NMR}\left(\mathrm{CDCl}_{3}, 101 \mathrm{MHz}\right)$ of compound $7 \mathbf{b}$

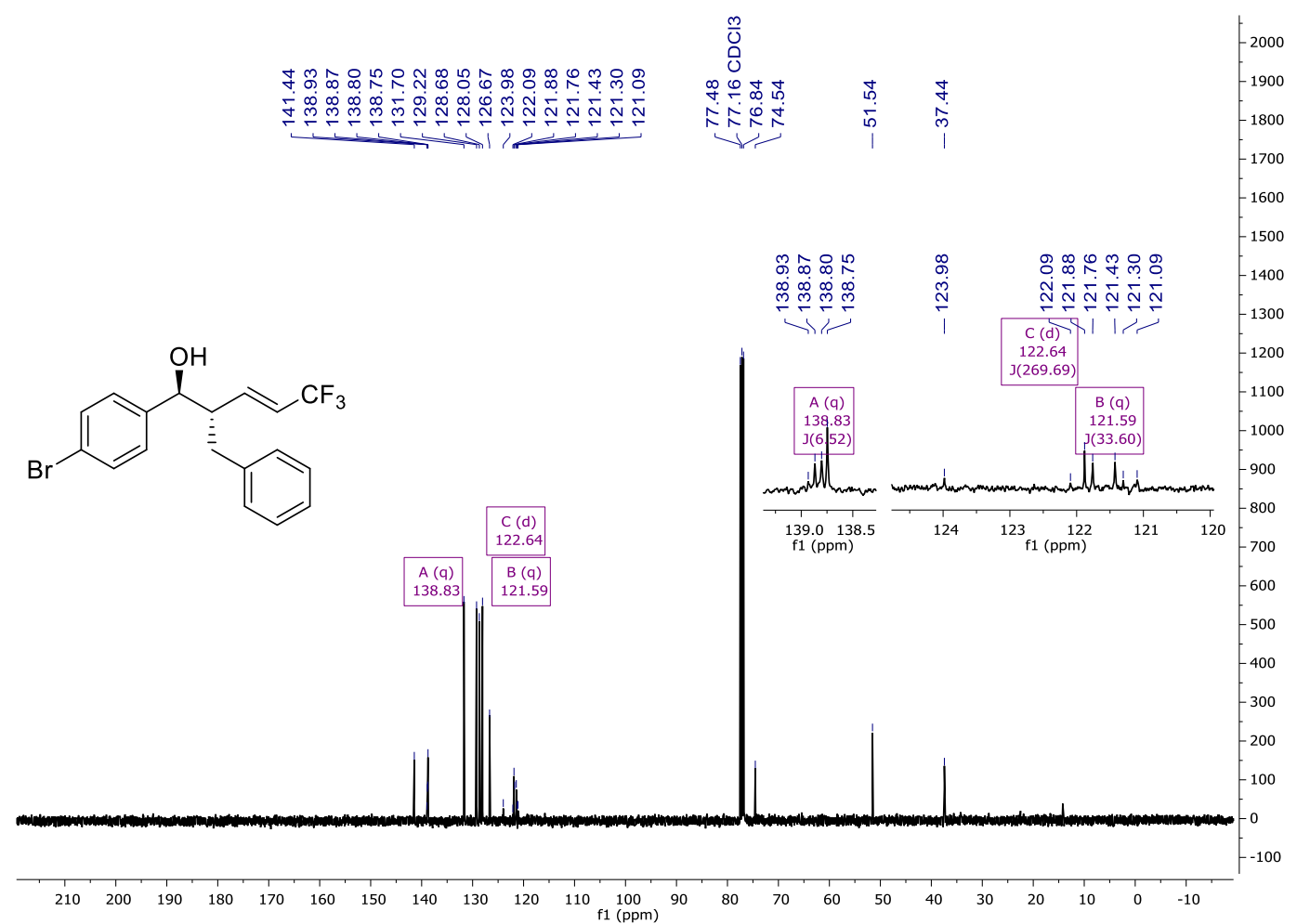


${ }^{19} \mathrm{~F} \mathrm{NMR}\left(\mathrm{CDCl}_{3}, 377 \mathrm{MHz}\right)$ of compound $7 \mathbf{b}$

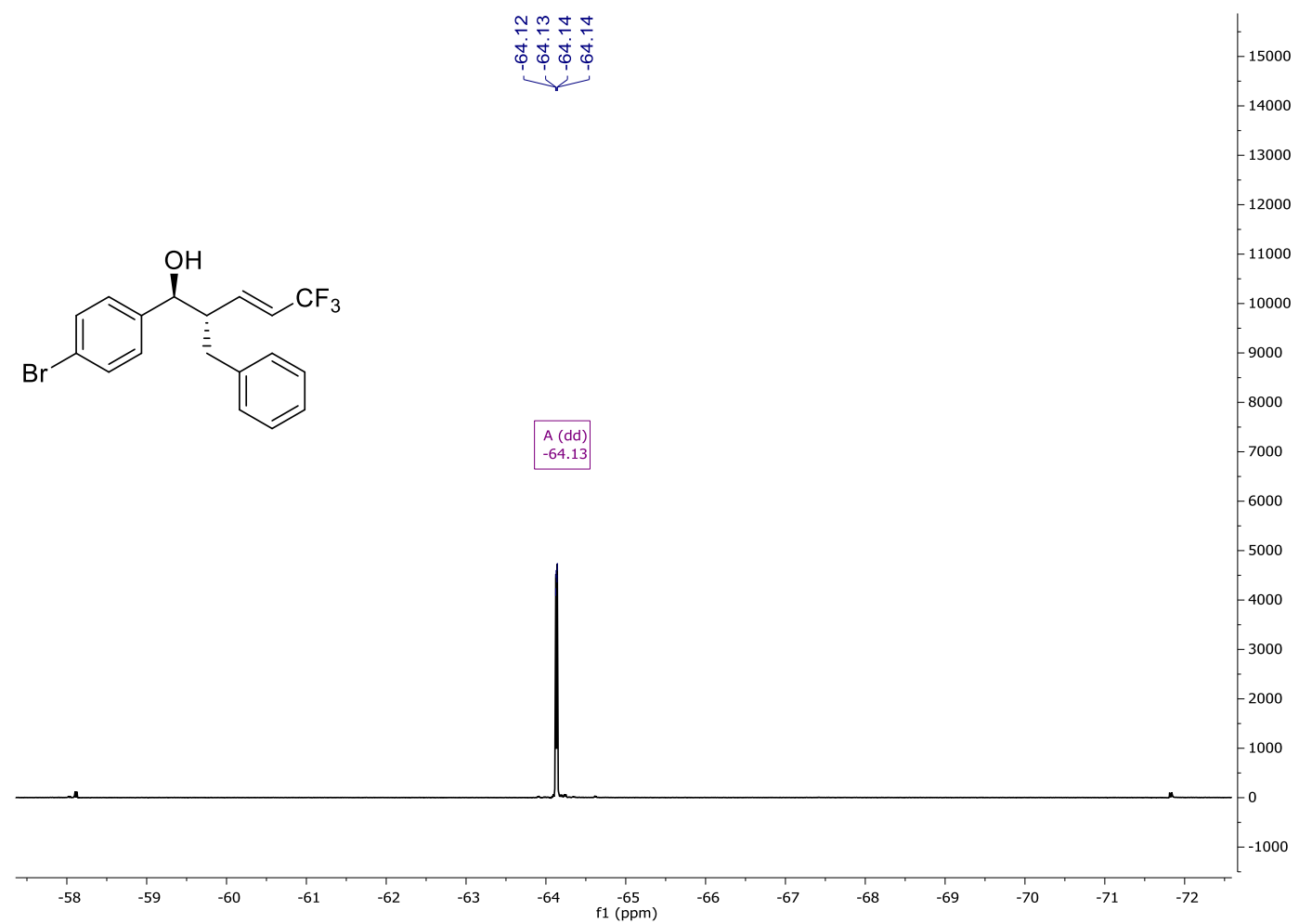

\section{${ }^{1} \mathrm{H} \mathrm{NMR}\left(\mathrm{CDCl}_{3}, 400 \mathrm{MHz}\right)$ of compound 7c}

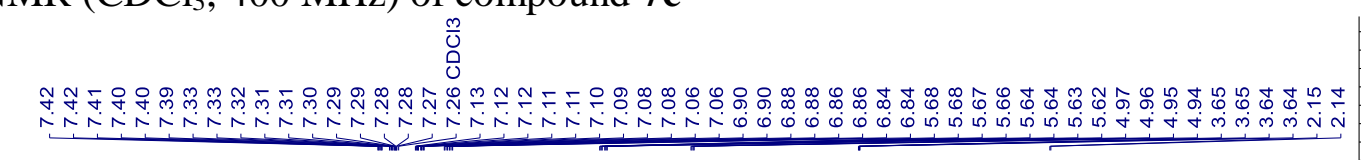

$-23000000$

$-22000000$

$-21000000$

20000000

$-19000000$

$-18000000$

$-17000000$

$-16000000$

CF

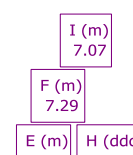

\begin{tabular}{l|}
$I(m)$ \\
7.07
\end{tabular}

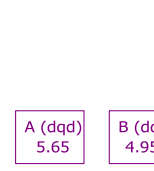

\begin{tabular}{|c|}
\hline C (ddt) \\
3.65 \\
\hline
\end{tabular}

\begin{tabular}{|l|}
\hline$D(d)$ \\
2.15 \\
\hline
\end{tabular}

$-15000000$

$-14000000$

$-13000000$

$-12000000$

$-11000000$

$-10000000$

$-9000000$

$-8000000$

7000000

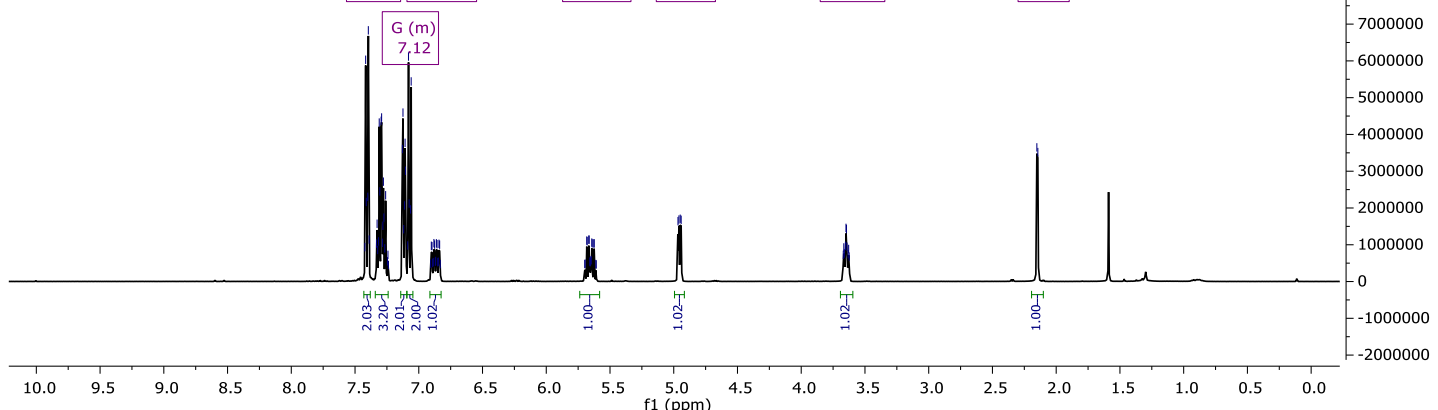


${ }^{13} \mathrm{C}$ NMR $\left(\mathrm{CDCl}_{3}, 101 \mathrm{MHz}\right)$ of compound $\mathbf{7 c}$

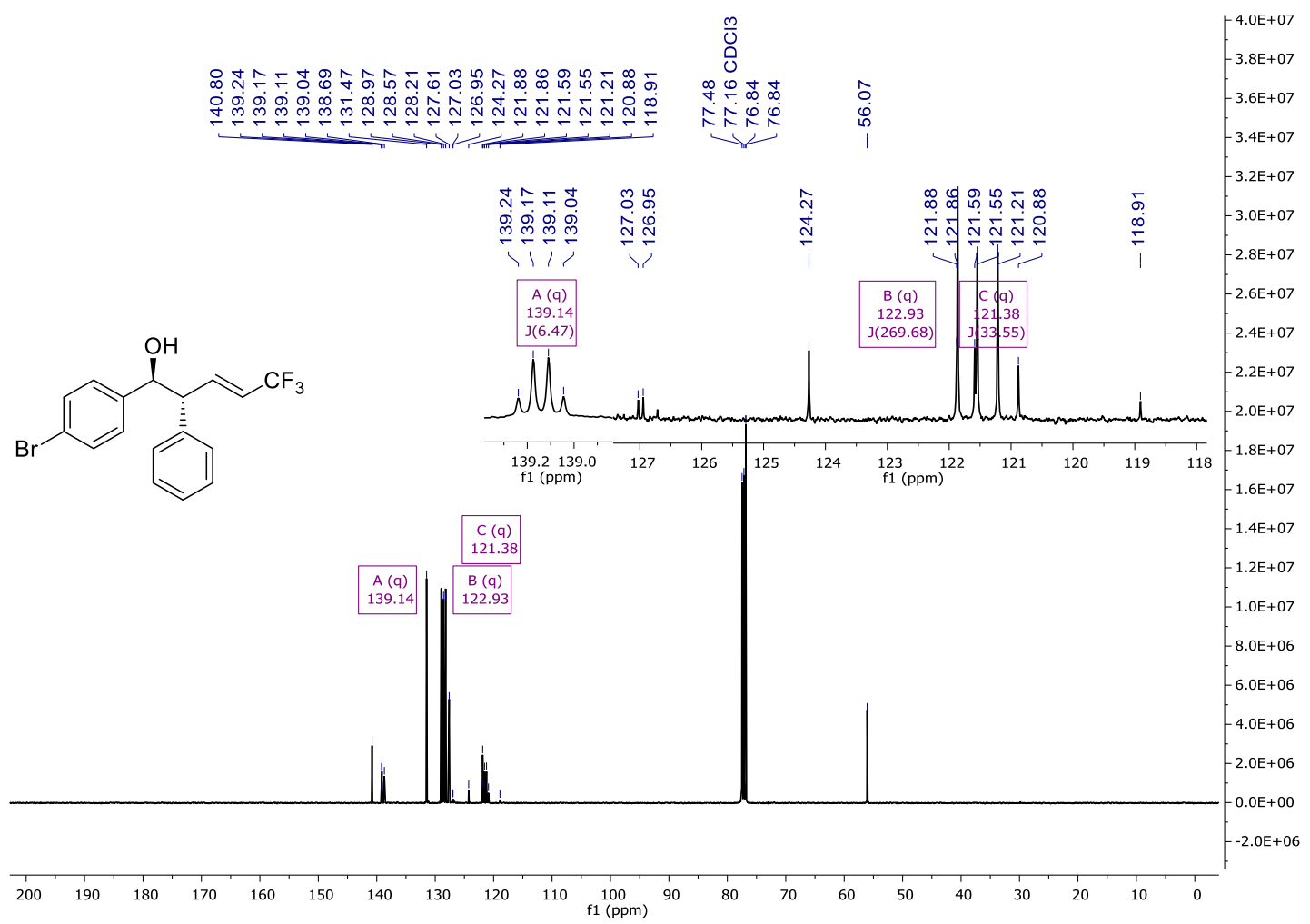

${ }^{19} \mathrm{~F}$ NMR $\left(\mathrm{CDCl}_{3}, 377 \mathrm{MHz}\right)$ of compound $7 \mathbf{c}$

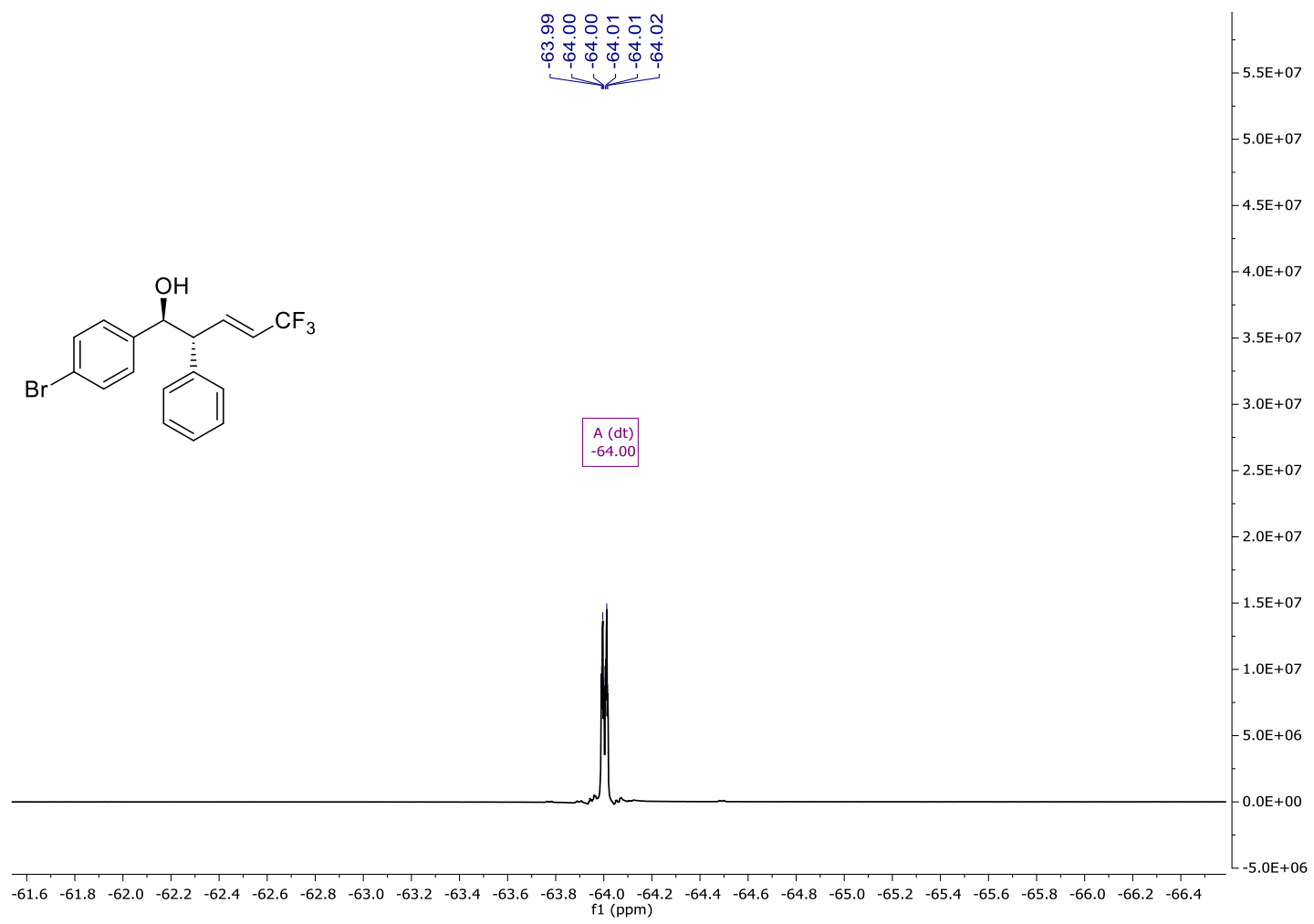




\section{${ }^{1} \mathrm{H} \mathrm{NMR}\left(\mathrm{CDCl}_{3}, 400 \mathrm{MHz}\right)$ of compound 7d}

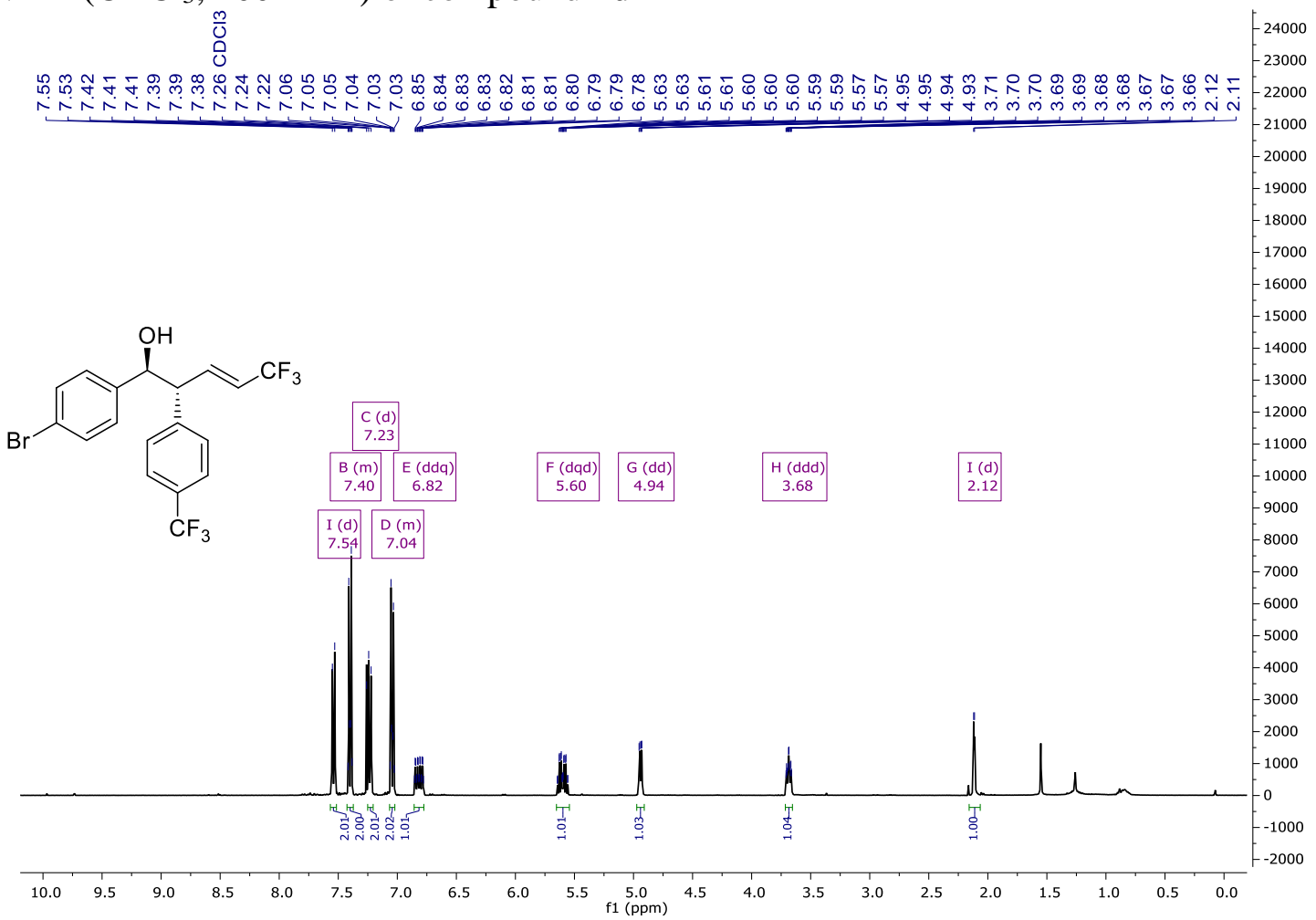

${ }^{13} \mathrm{C} \mathrm{NMR}\left(\mathrm{CDCl}_{3}, 101 \mathrm{MHz}\right)$ of compound $\mathbf{7 d}$

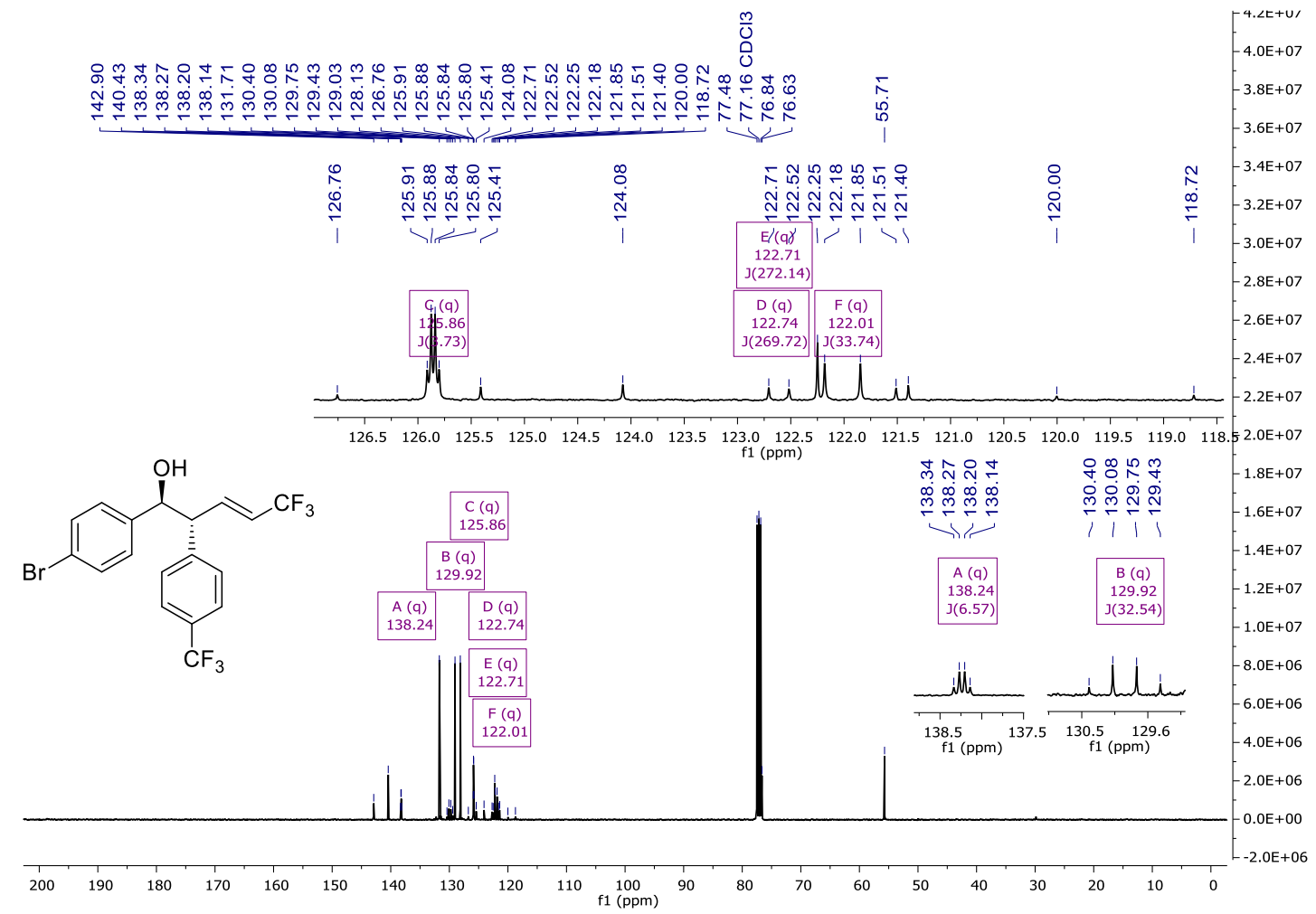


${ }^{19} \mathrm{~F} \mathrm{NMR}\left(\mathrm{CDCl}_{3}, 377 \mathrm{MHz}\right)$ of compound $7 \mathbf{d}$

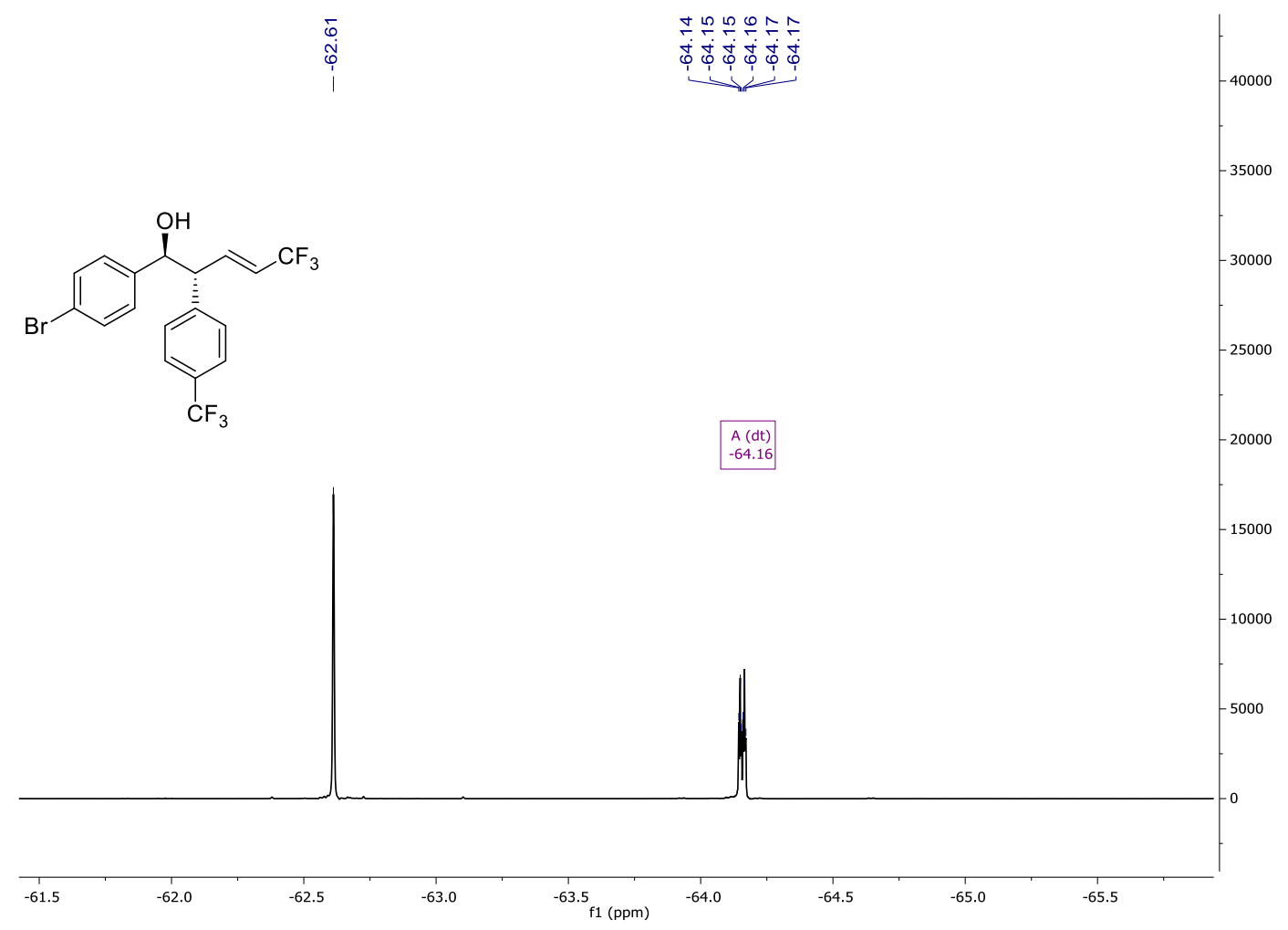

${ }^{1} \mathrm{H}$ NMR $\left(\mathrm{CDCl}_{3}, 400 \mathrm{MHz}\right)$ of compound $7 \mathbf{e}$

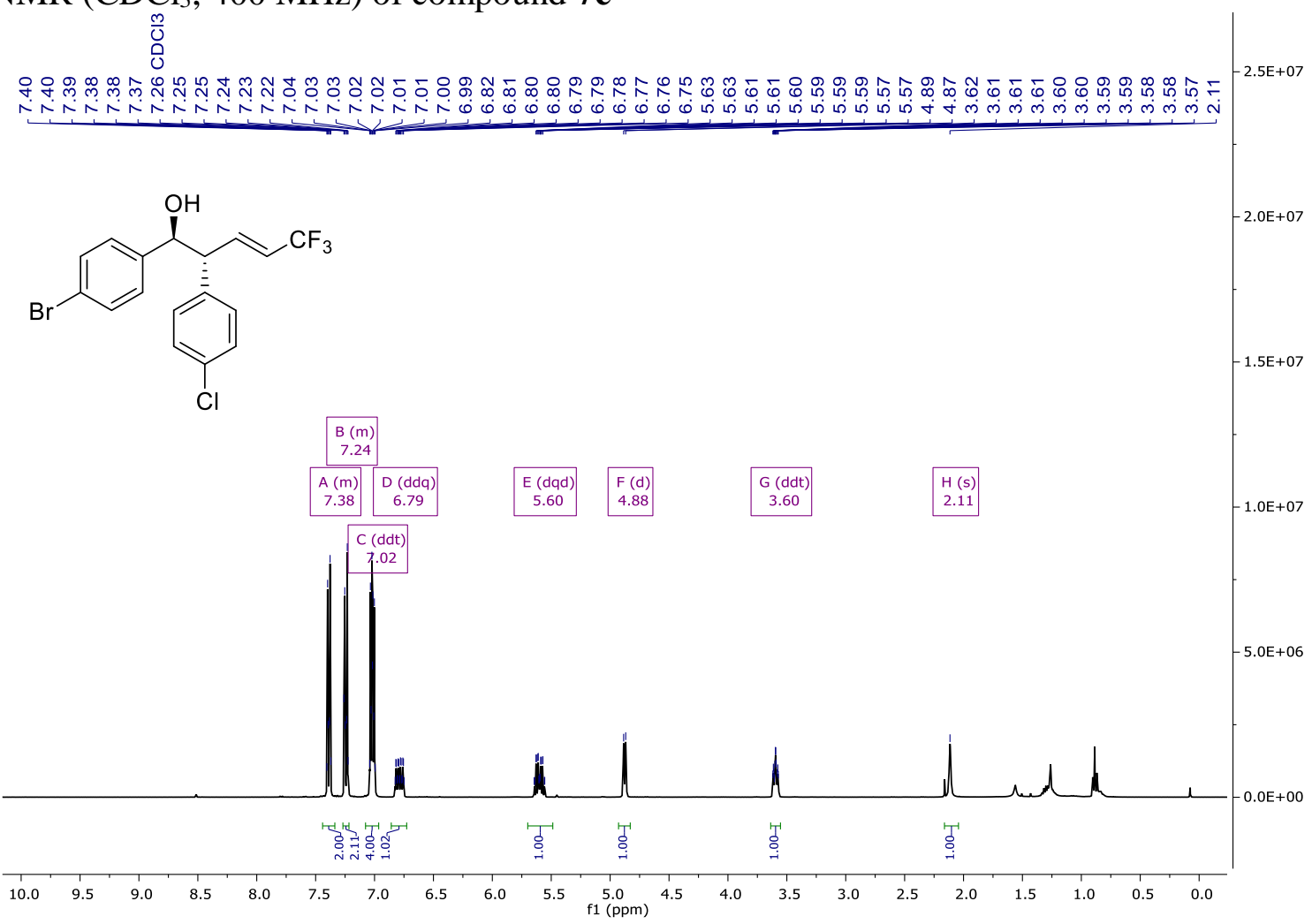


${ }^{13} \mathrm{C}$ NMR $\left(\mathrm{CDCl}_{3}, 101 \mathrm{MHz}\right)$ of compound $7 \mathbf{e}$

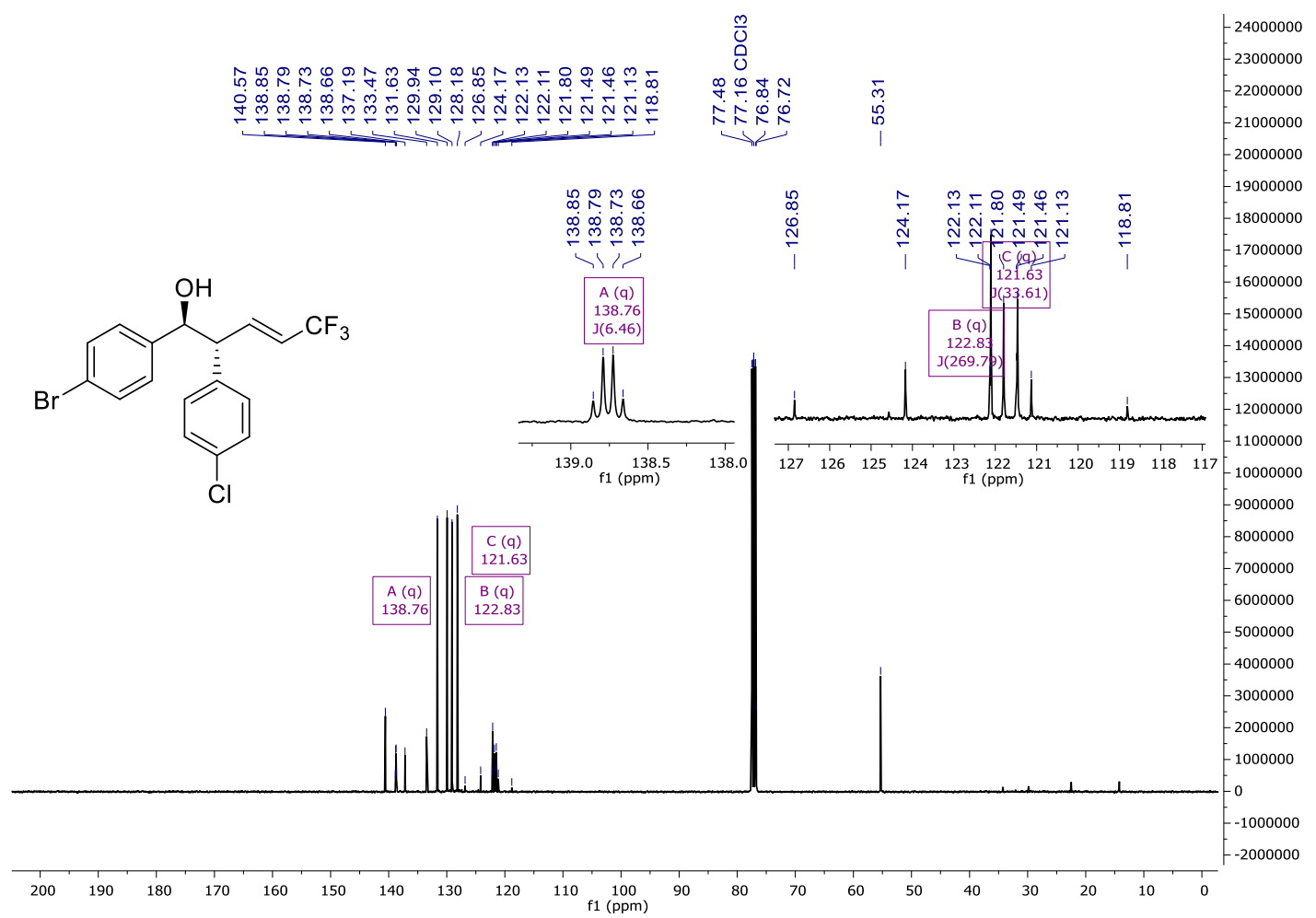

${ }^{19} \mathrm{~F} \mathrm{NMR}\left(\mathrm{CDCl}_{3}, 377 \mathrm{MHz}\right)$ of compound $7 \mathbf{e}$

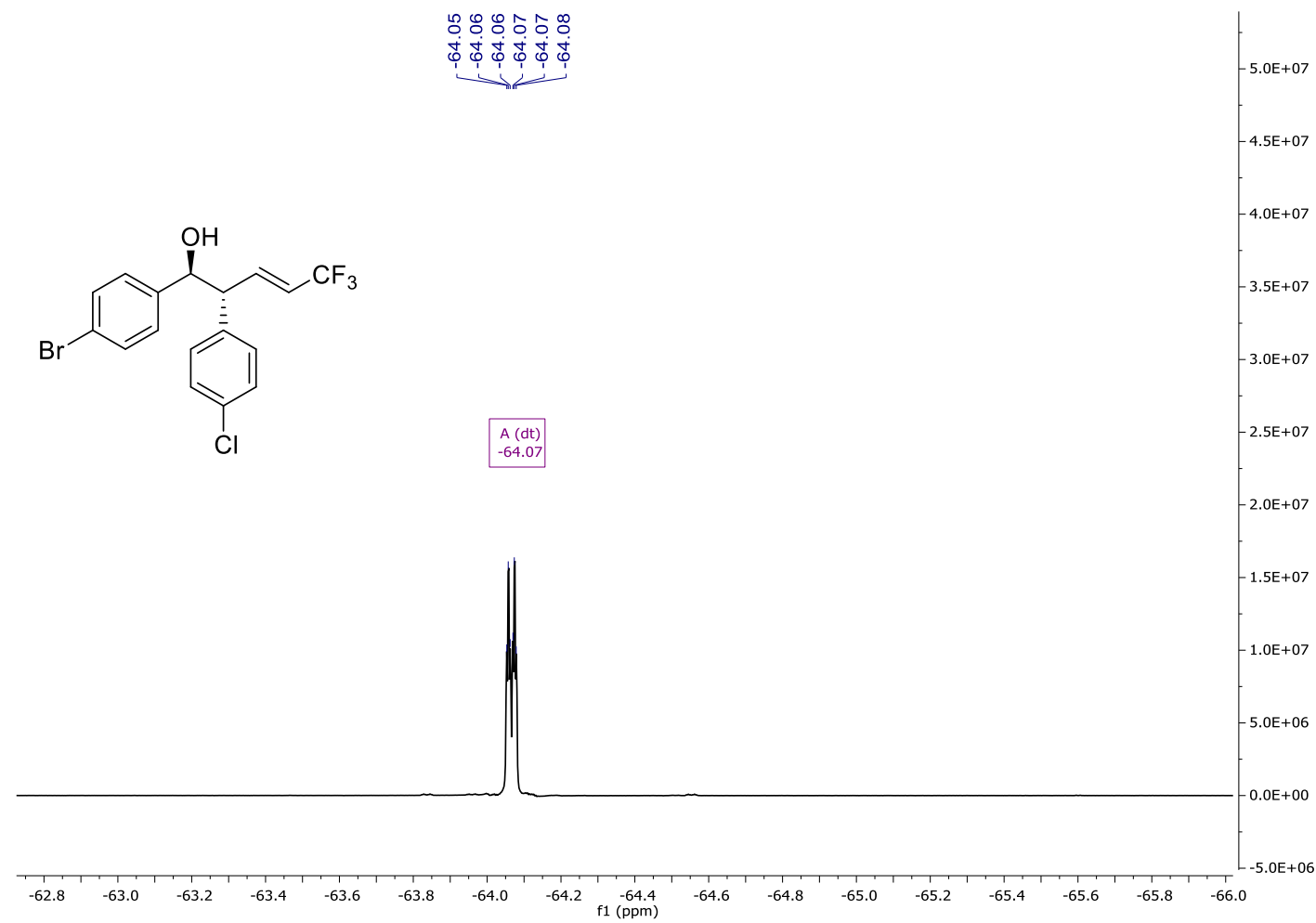


${ }^{1} \mathrm{H} \mathrm{NMR}\left(\mathrm{CDCl}_{3}, 400 \mathrm{MHz}\right)$ of compound $7 \mathbf{f}$

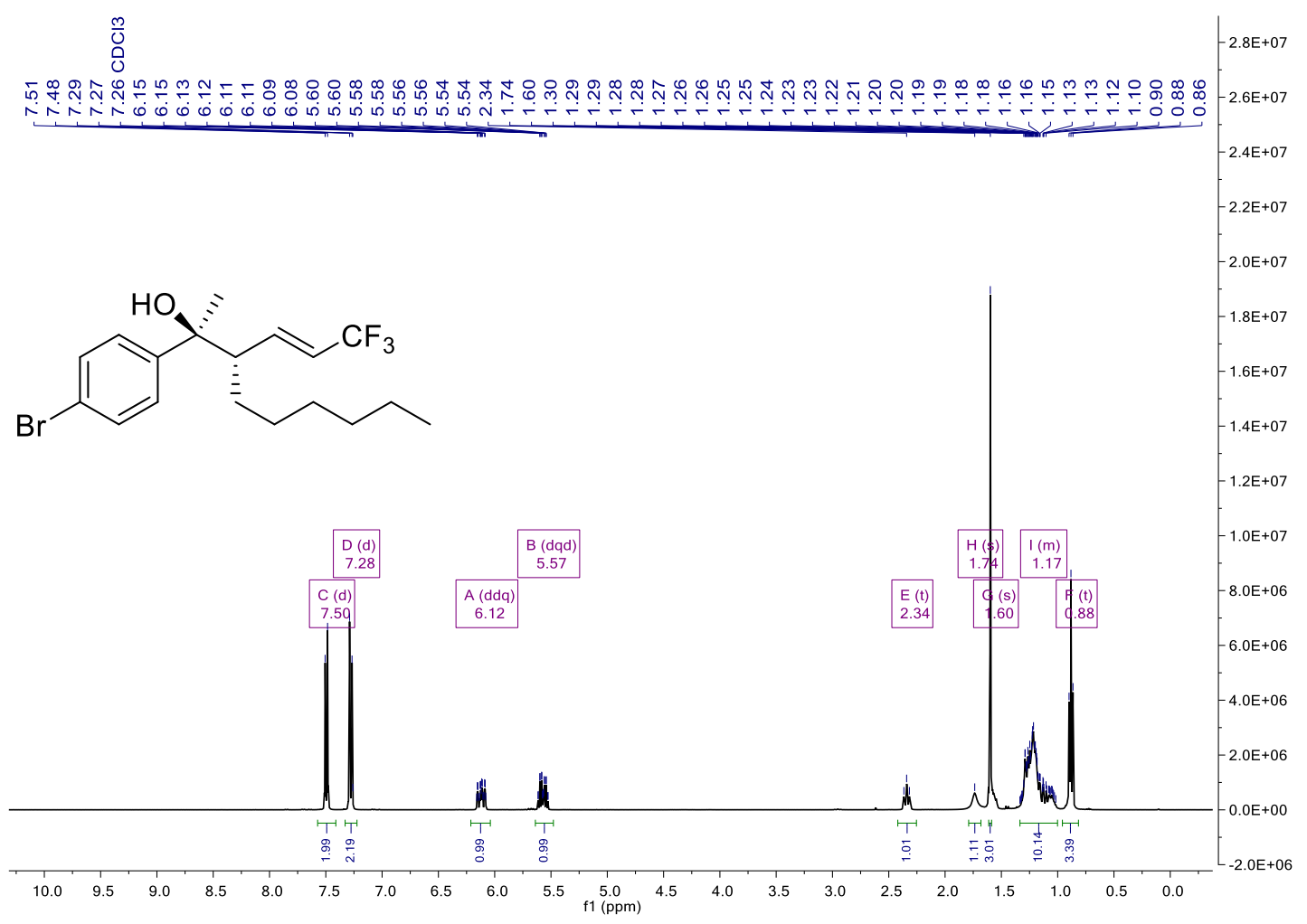

${ }^{13} \mathrm{C} \mathrm{NMR}\left(\mathrm{CDCl}_{3}, 101 \mathrm{MHz}\right)$ of compound $7 \mathbf{f}$

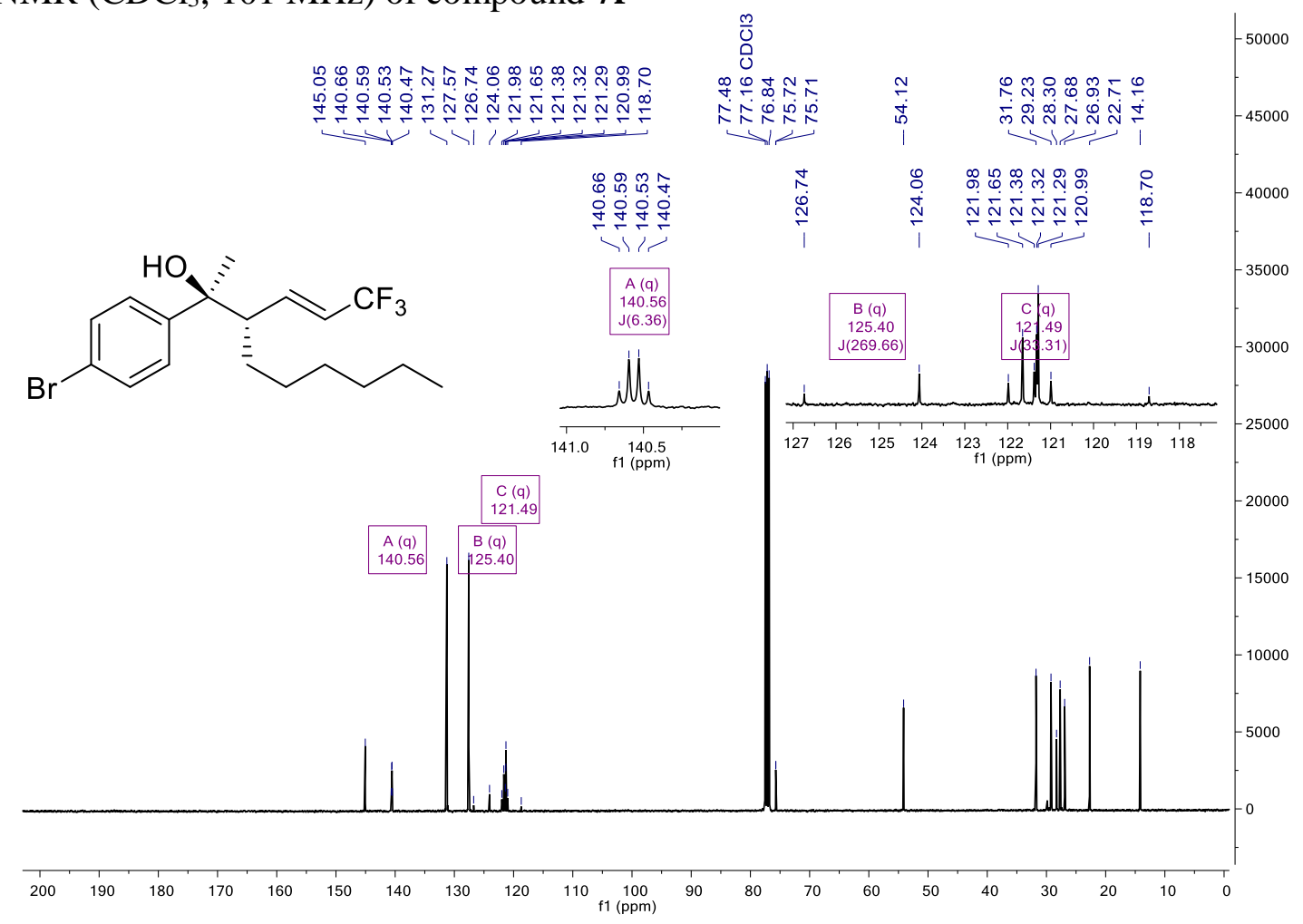


${ }^{19} \mathrm{~F} \mathrm{NMR}\left(\mathrm{CDCl}_{3}, 377 \mathrm{MHz}\right)$ of compound $7 \mathbf{f}$

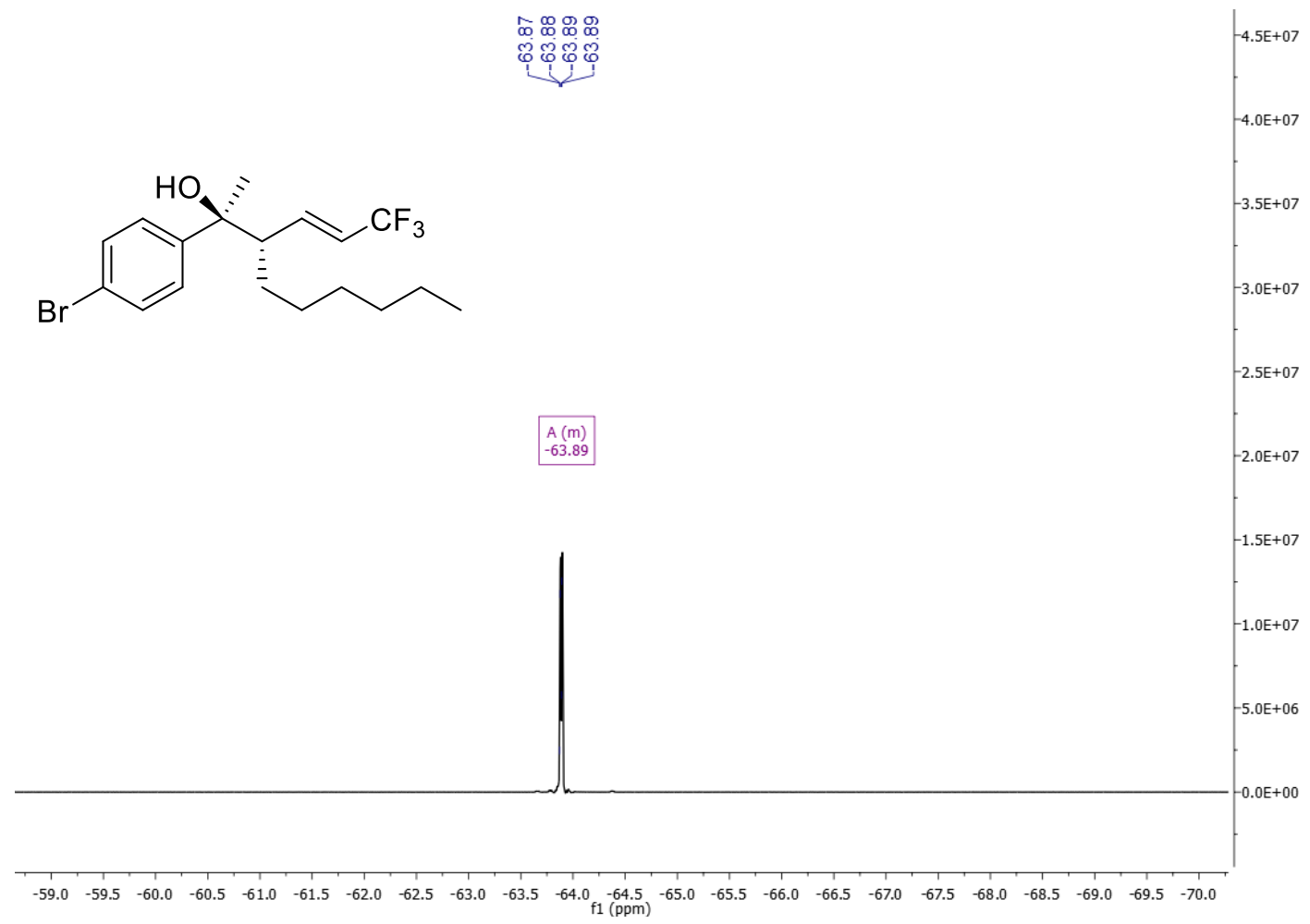

${ }^{1} \mathrm{H} \mathrm{NMR}\left(\mathrm{CDCl}_{3}, 400 \mathrm{MHz}\right)$ of compound $7 \mathbf{g}$

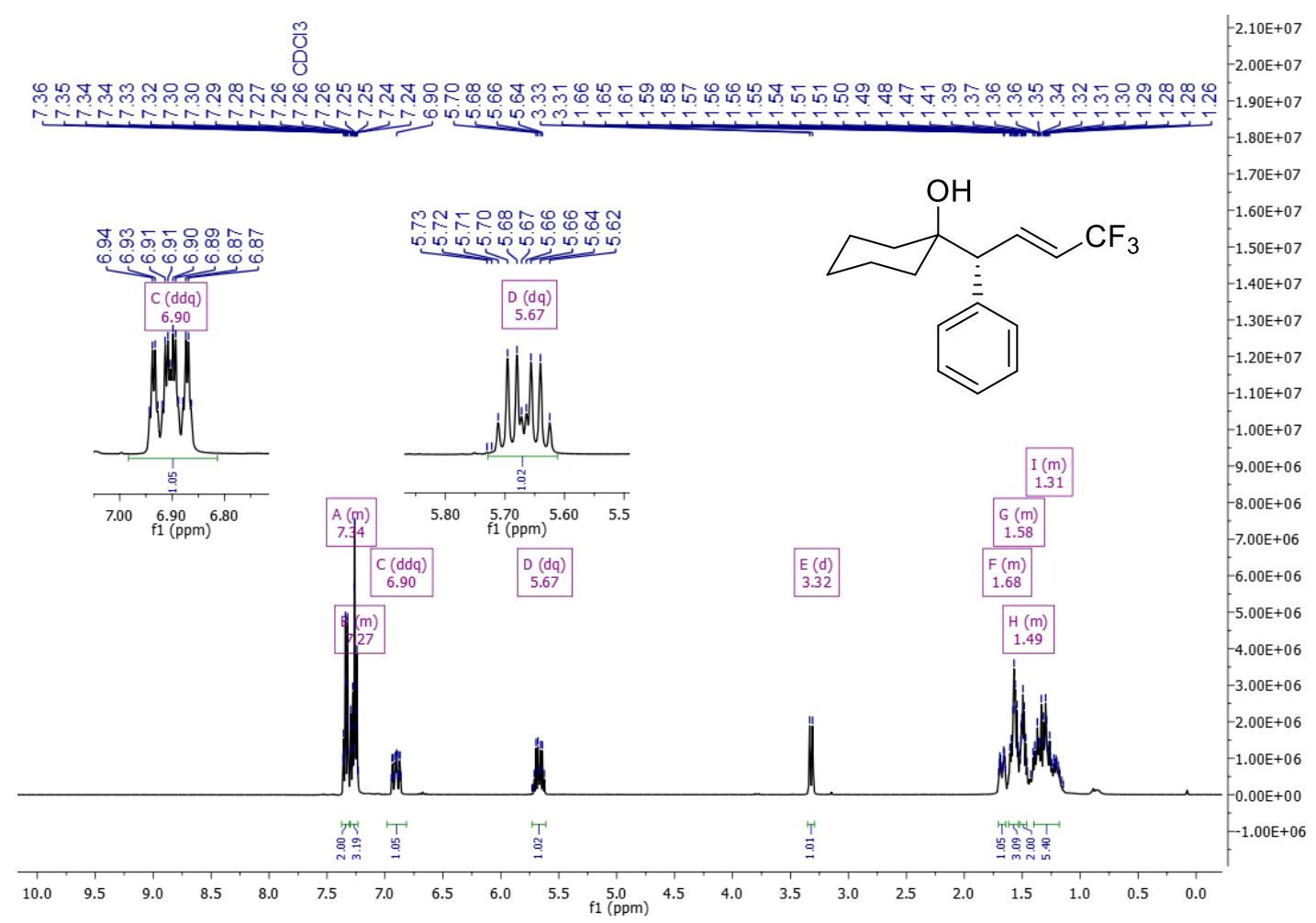


${ }^{13} \mathrm{C} \mathrm{NMR}\left(\mathrm{CDCl}_{3}, 101 \mathrm{MHz}\right)$ of compound $\mathbf{7 g}$

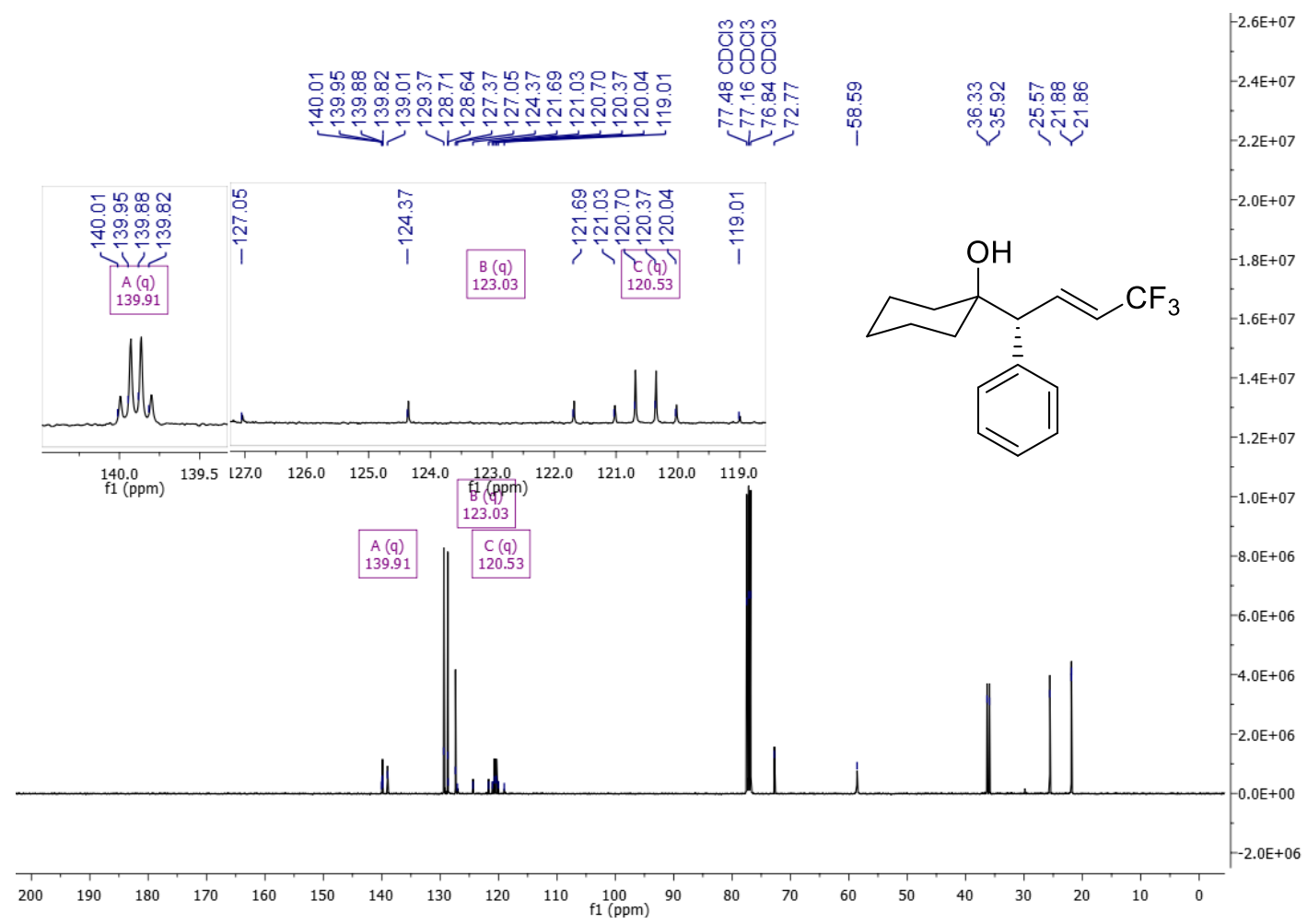

${ }^{19} \mathrm{~F} \mathrm{NMR}\left(\mathrm{CDCl}_{3}, 377 \mathrm{MHz}\right)$ of compound $\mathbf{7 g}$

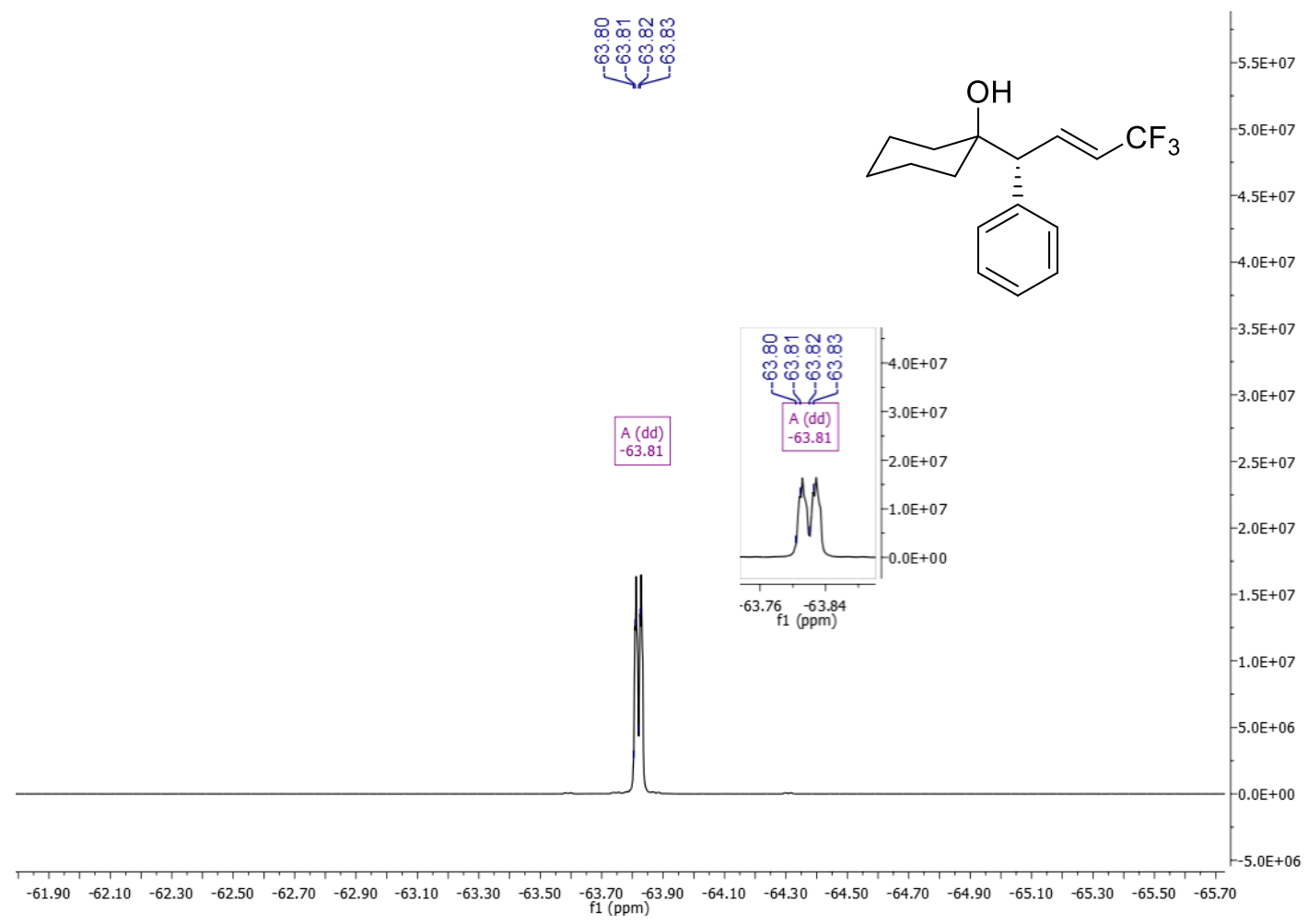


${ }^{1} \mathrm{H} \mathrm{NMR}\left(\mathrm{CDCl}_{3}, 400 \mathrm{MHz}\right)$ of compound $\mathbf{7 h}$

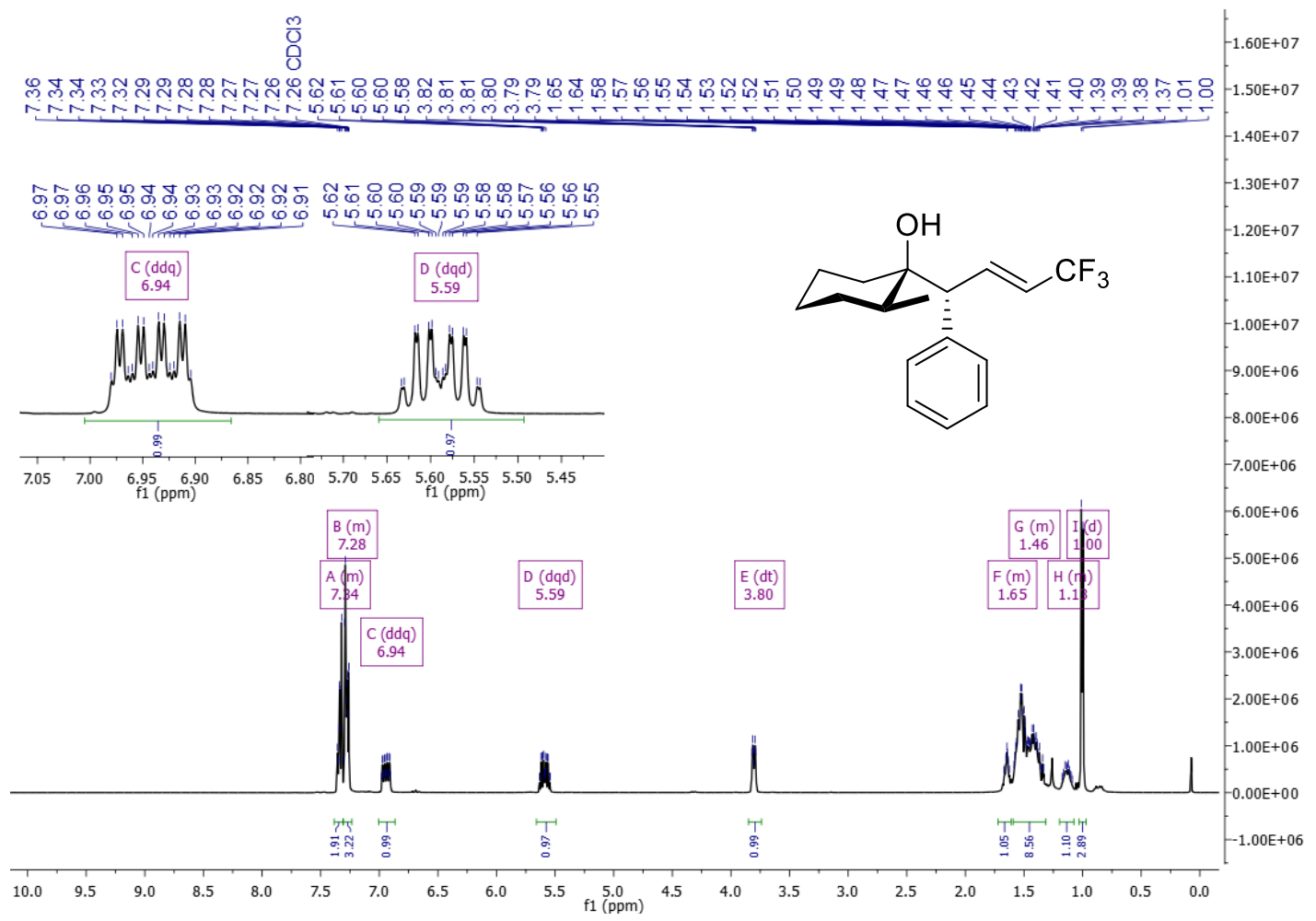

$\left.{ }^{13} \mathrm{C} \mathrm{NMR} \mathrm{(CDCl} 3,101 \mathrm{MHz}\right)$ of compound $\mathbf{7 h}$

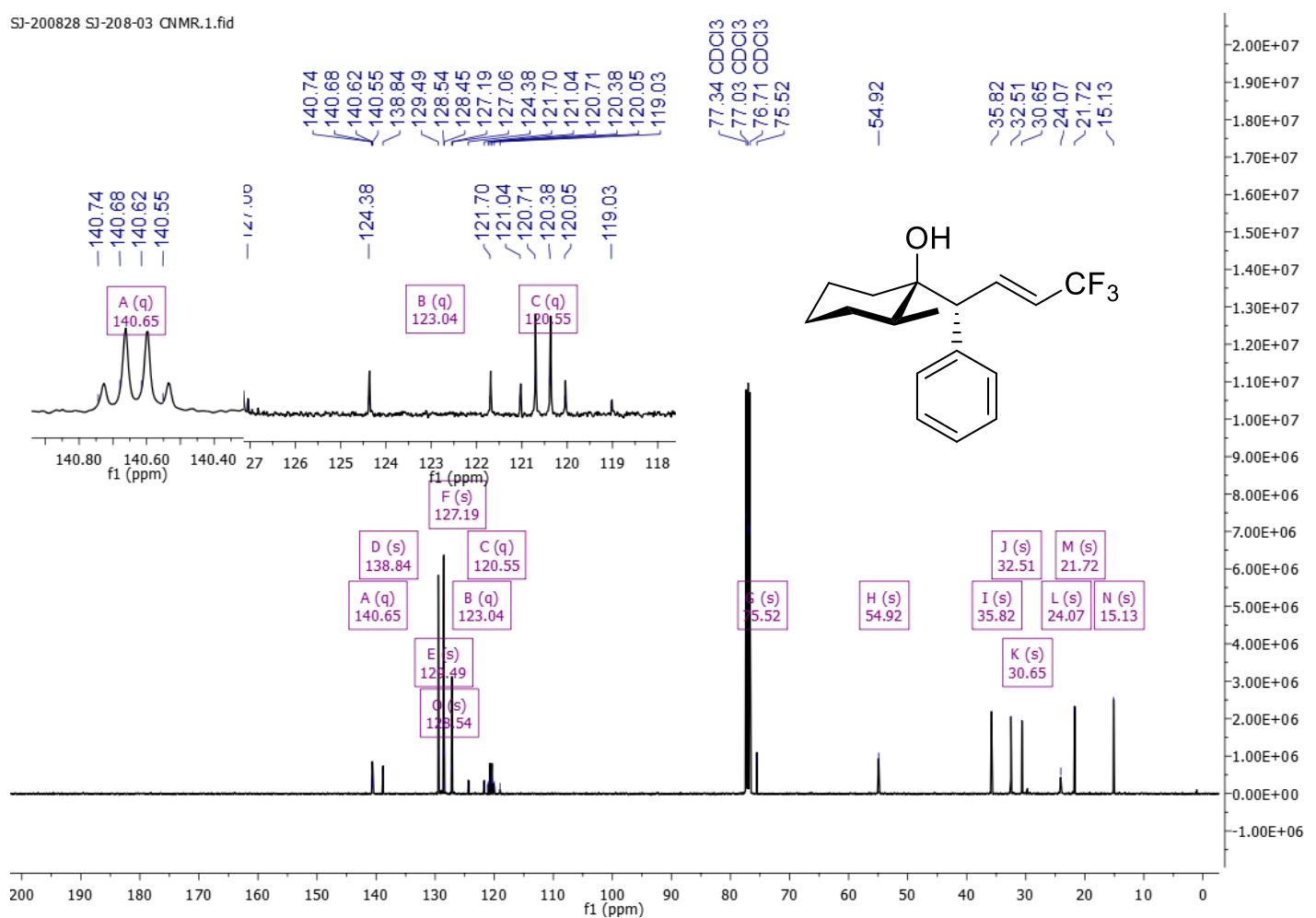


${ }^{19} \mathrm{~F} \mathrm{NMR}\left(\mathrm{CDCl}_{3}, 377 \mathrm{MHz}\right)$ of compound $7 \mathbf{h}$

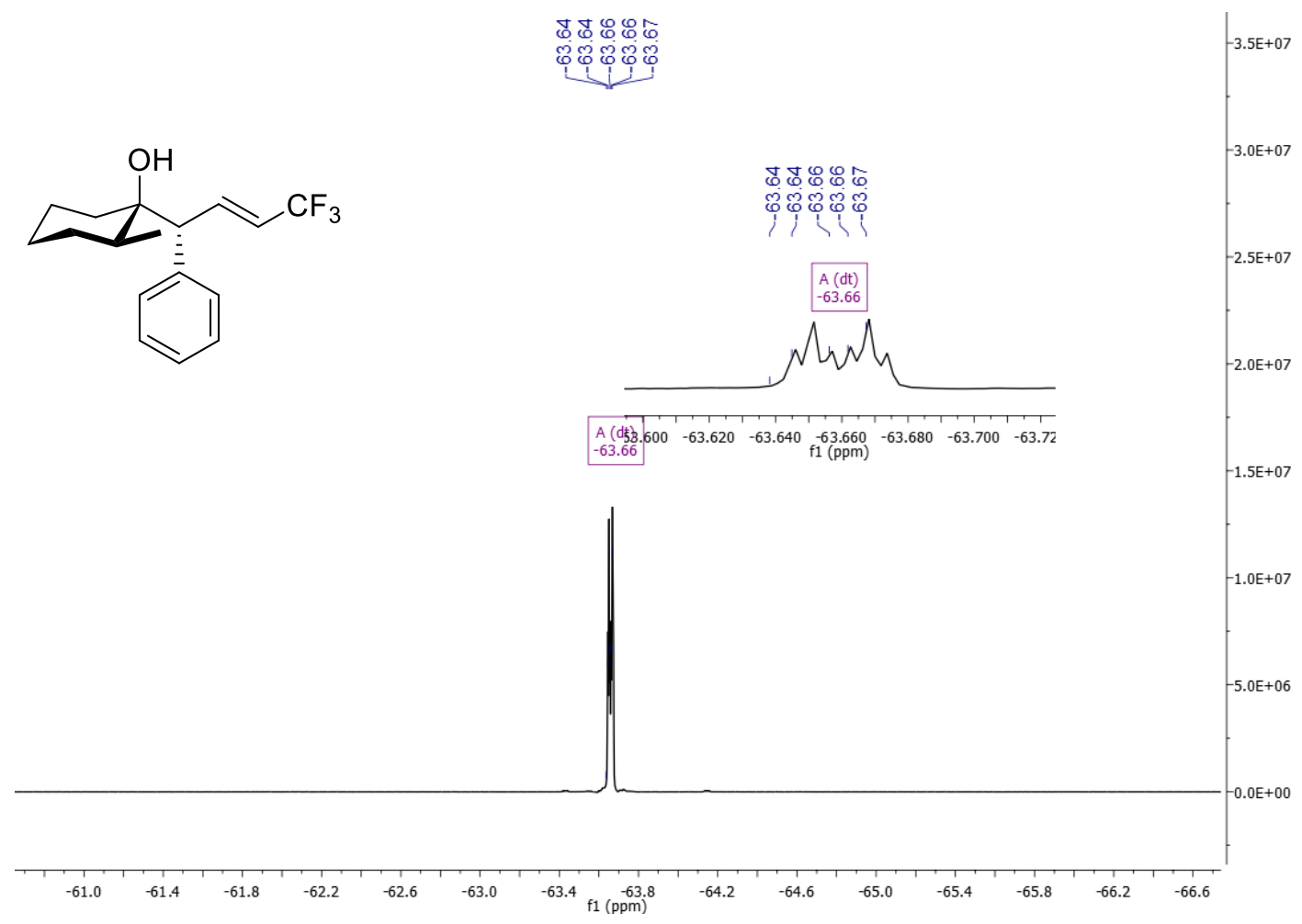

${ }^{1} \mathrm{H} \mathrm{NMR}\left(\mathrm{CDCl}_{3}, 400 \mathrm{MHz}\right)$ of compound $\mathbf{7 i}$

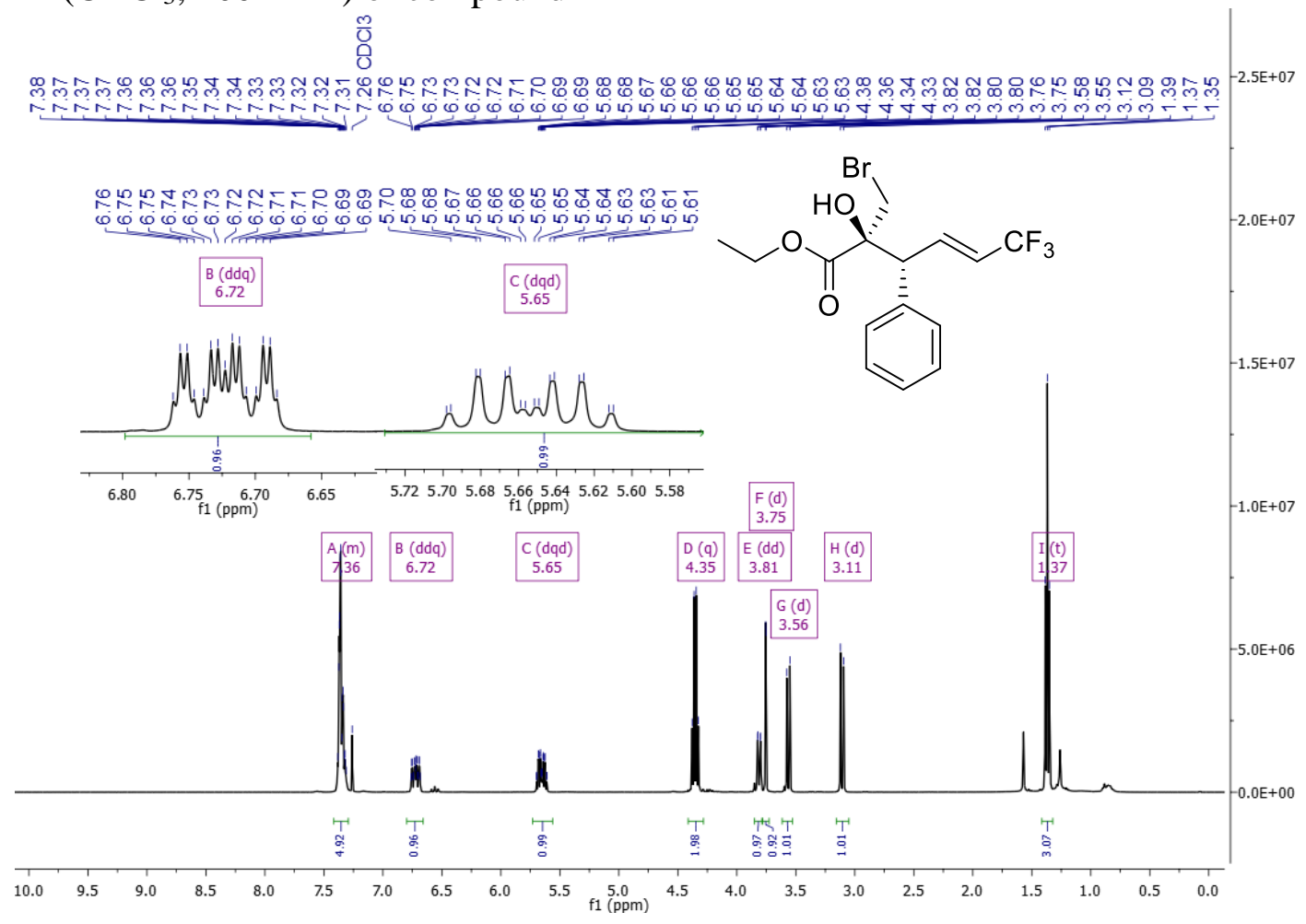


${ }^{13} \mathrm{C} \mathrm{NMR}\left(\mathrm{CDCl}_{3}, 101 \mathrm{MHz}\right)$ of compound $7 \mathbf{i}$

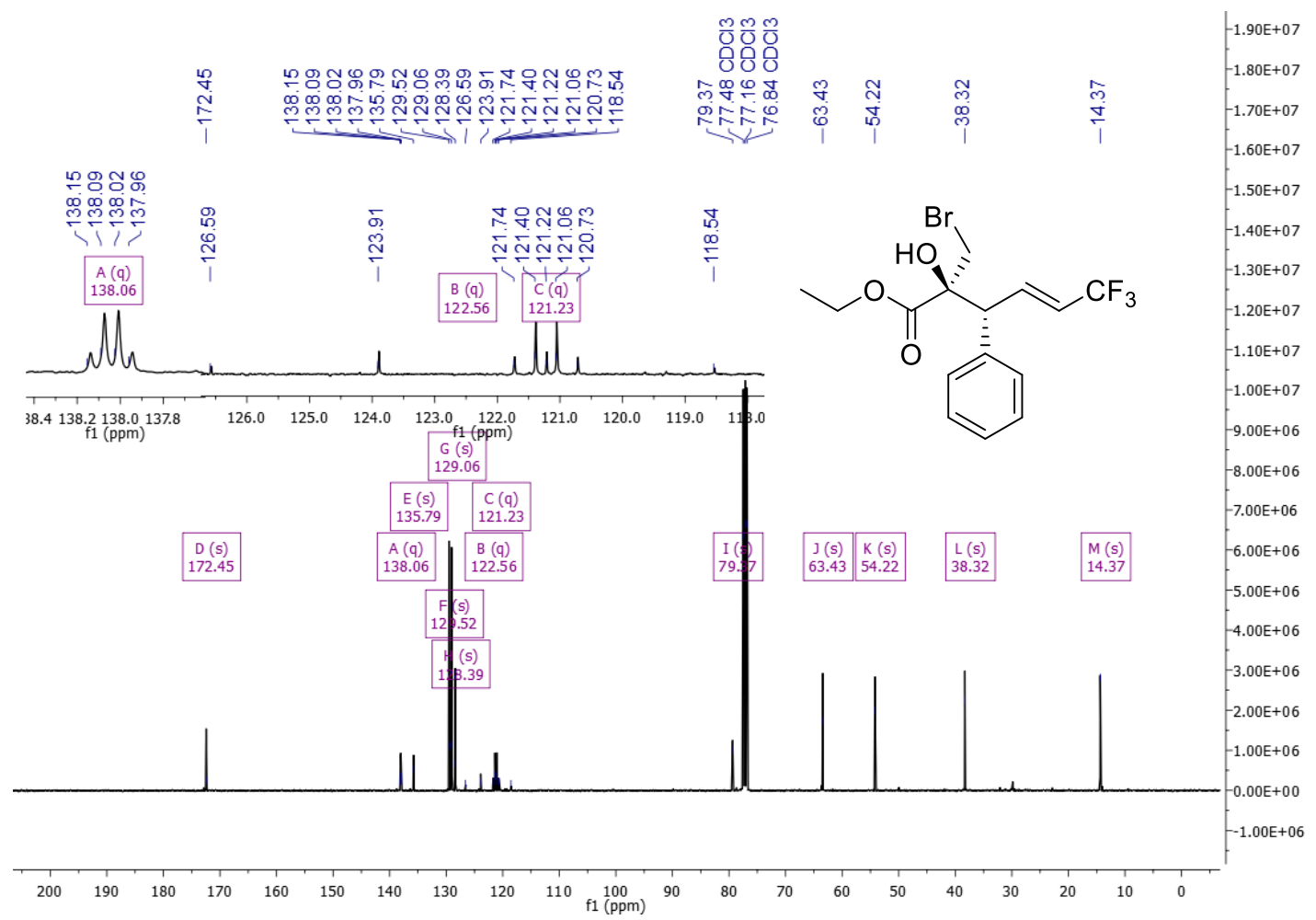

${ }^{19} \mathrm{~F} \mathrm{NMR}\left(\mathrm{CDCl}_{3}, 377 \mathrm{MHz}\right)$ of compound $7 \mathbf{i}$

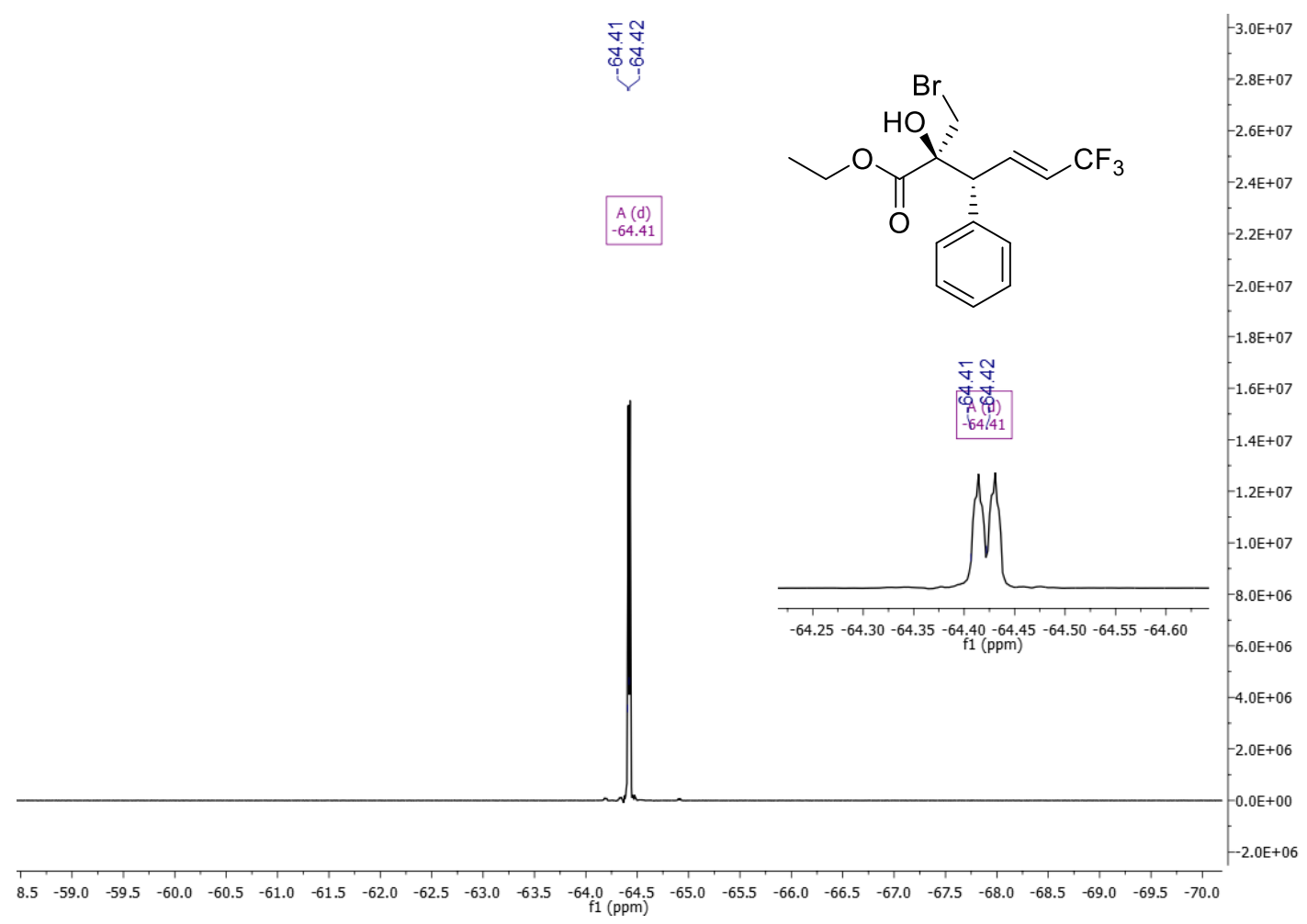


${ }^{1} \mathrm{H}$ NMR $\left(\mathrm{CDCl}_{3}, 400 \mathrm{MHz}\right)$ of compound $\mathbf{7} \mathbf{j}$

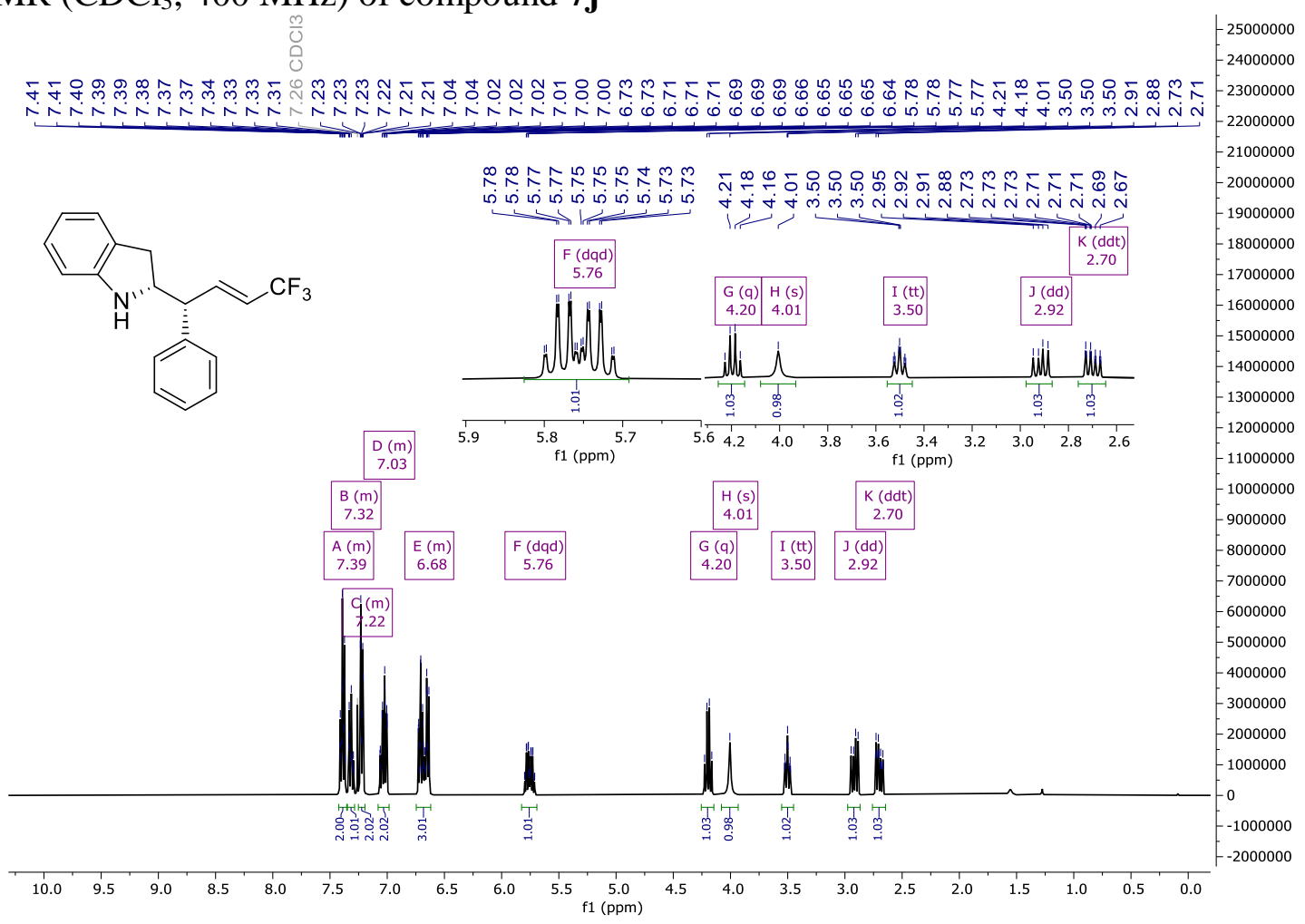

\section{${ }^{13} \mathrm{C}$ NMR $\left(\mathrm{CDCl}_{3}, 101 \mathrm{MHz}\right)$ of compound $\mathbf{7 j}$}

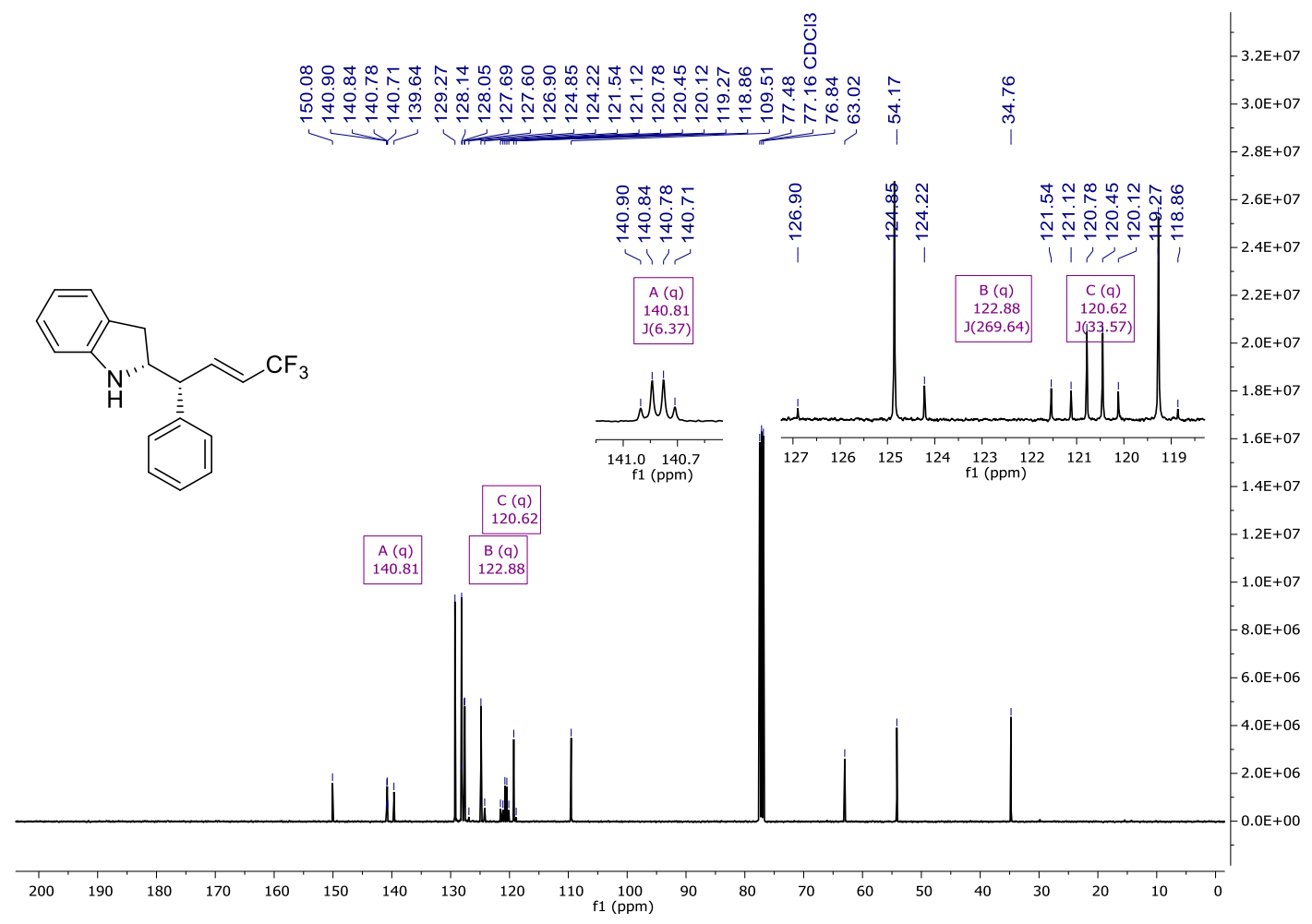


${ }^{19} \mathrm{~F} \mathrm{NMR}\left(\mathrm{CDCl}_{3}, 377 \mathrm{MHz}\right)$ of compound $7 \mathbf{i}$

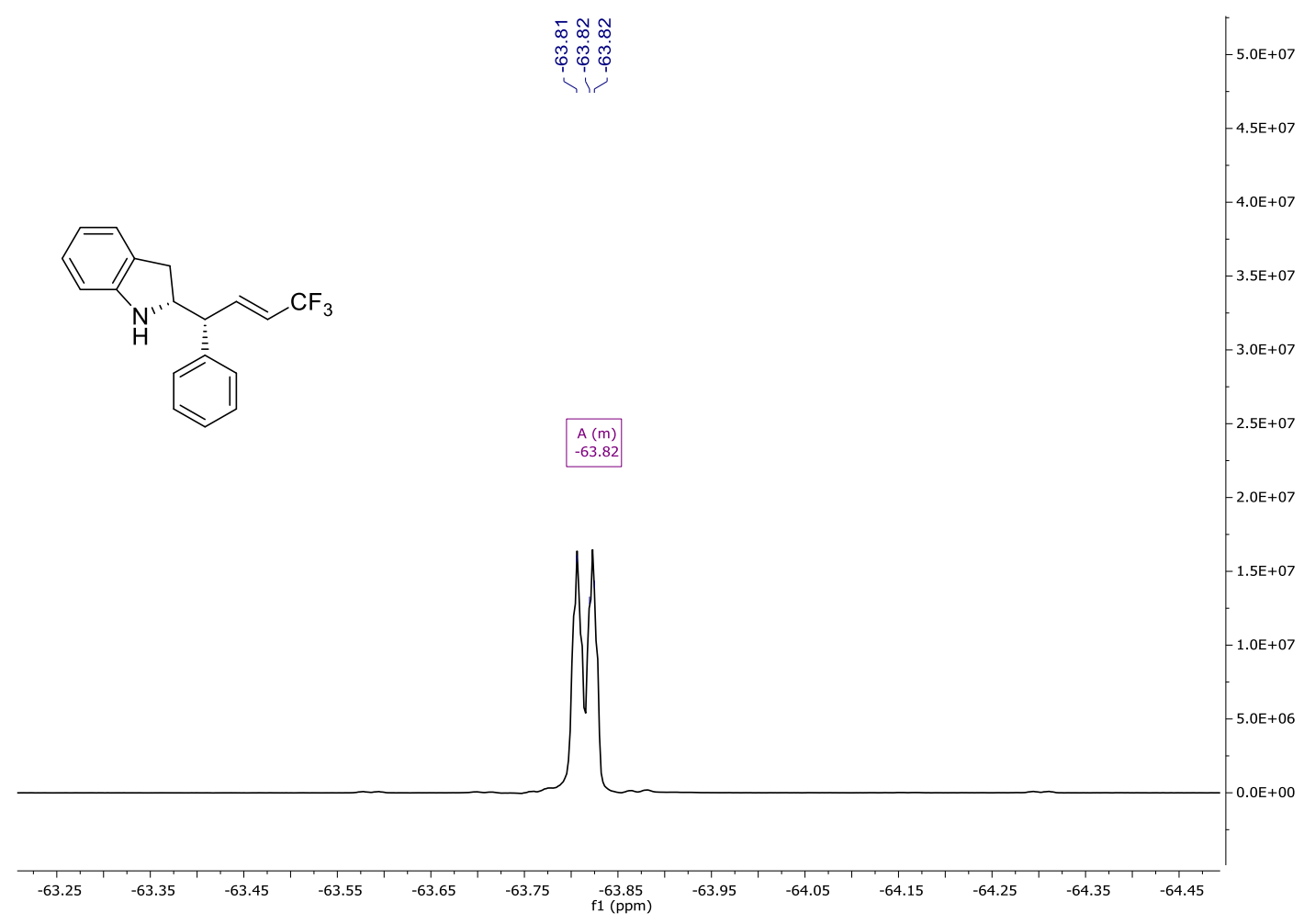

${ }^{1} \mathrm{H} \mathrm{NMR}\left(\mathrm{CDCl}_{3}, 400 \mathrm{MHz}\right)$ of compound $7 \mathbf{k}$

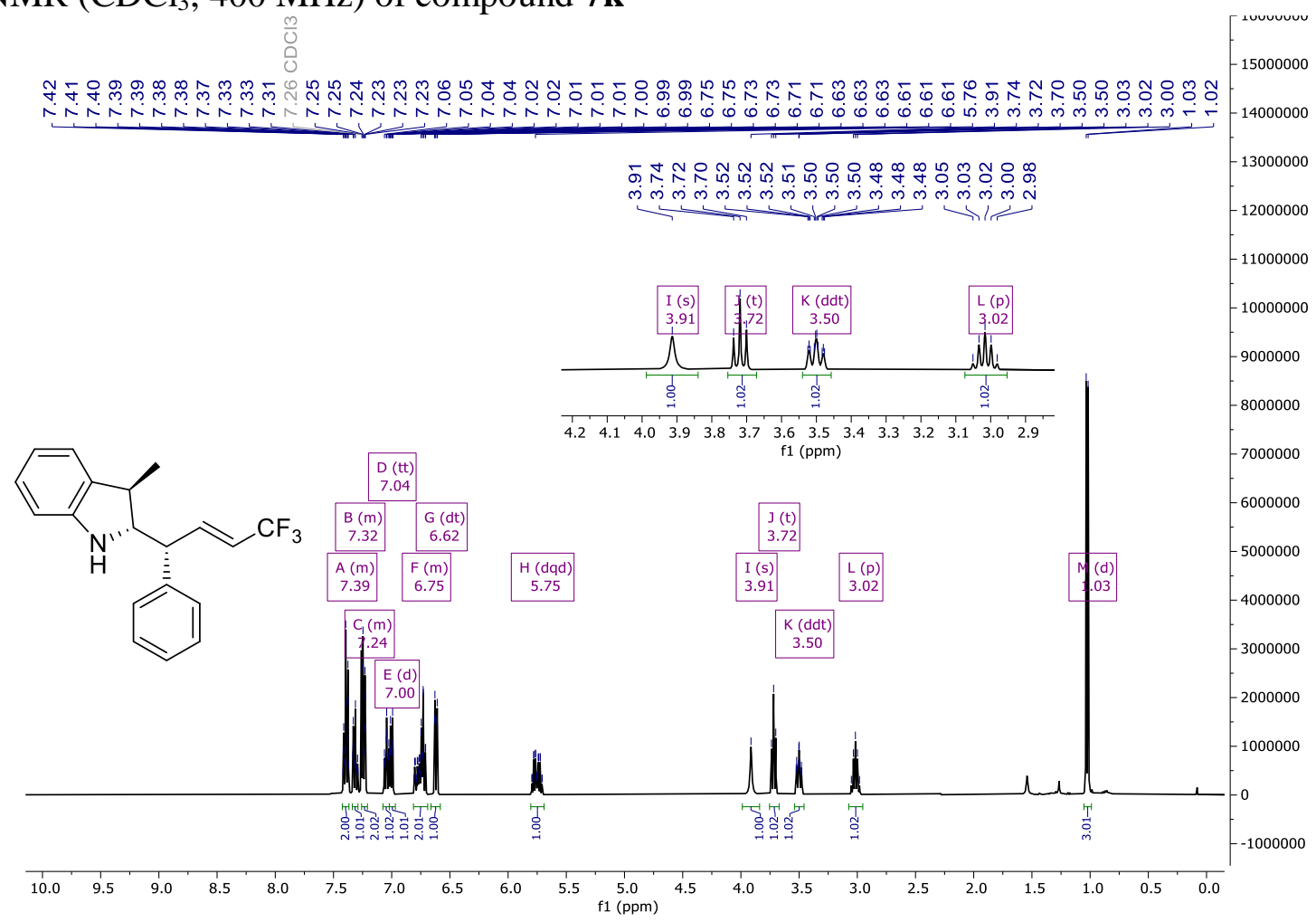


${ }^{13} \mathrm{C}$ NMR $\left(\mathrm{CDCl}_{3}, 126 \mathrm{MHz}\right)$ of compound $7 \mathbf{k}$

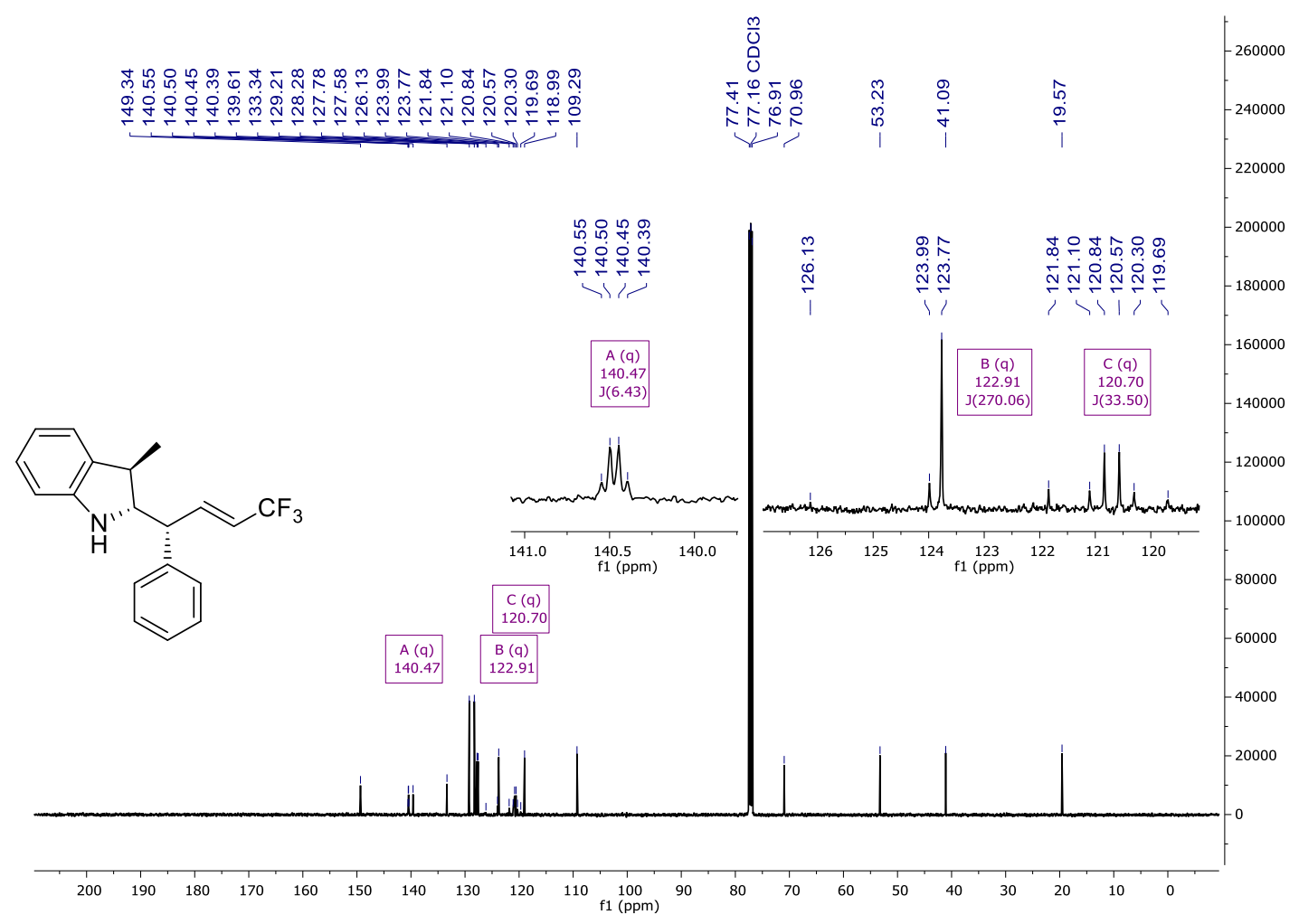

${ }^{19} \mathrm{~F}$ NMR $\left(\mathrm{CDCl}_{3}, 377 \mathrm{MHz}\right)$ of compound $7 \mathbf{k}$

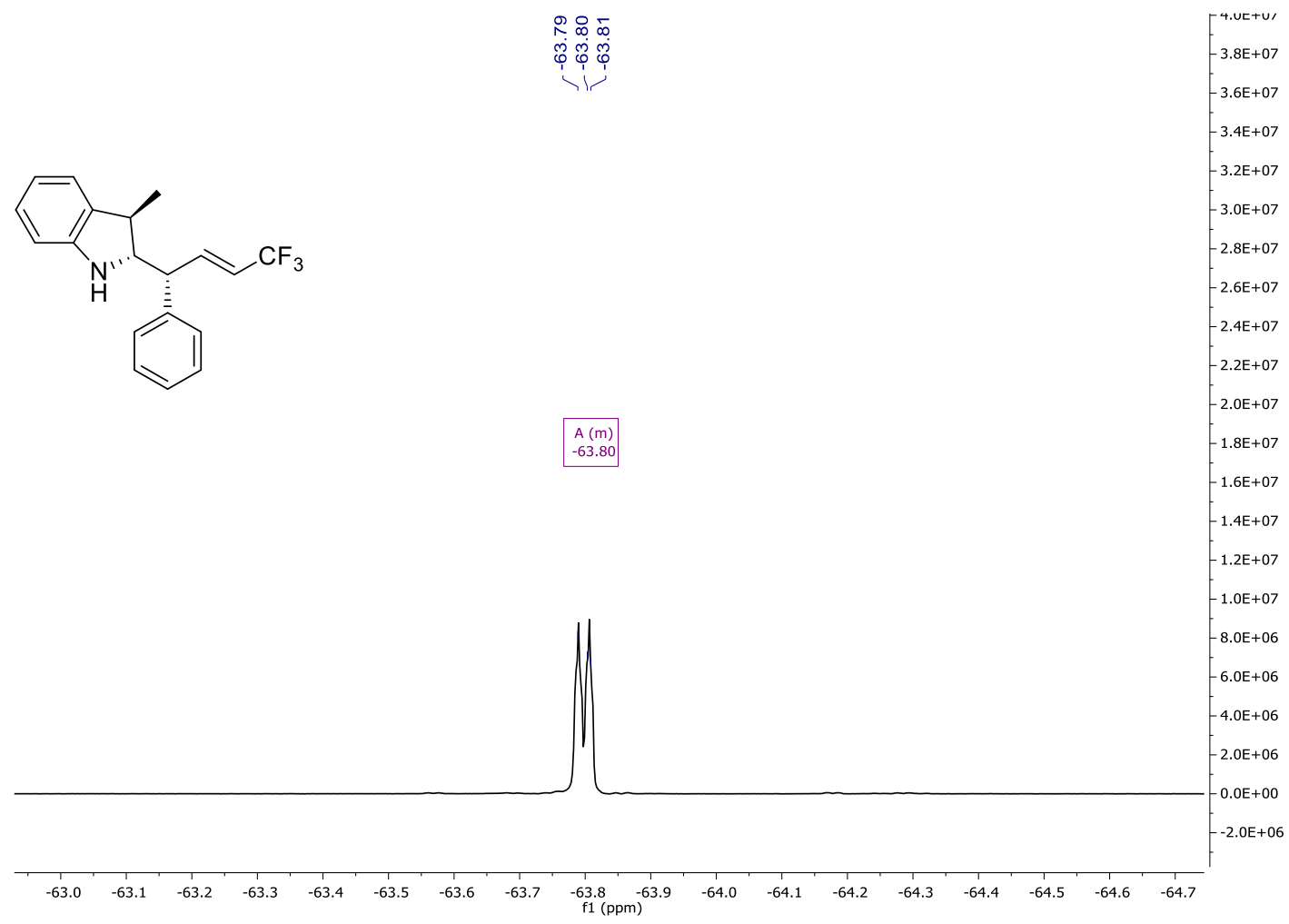


${ }^{1} \mathrm{H}$ NMR $\left(\mathrm{CDCl}_{3}, 400 \mathrm{MHz}\right)$ of compound 71

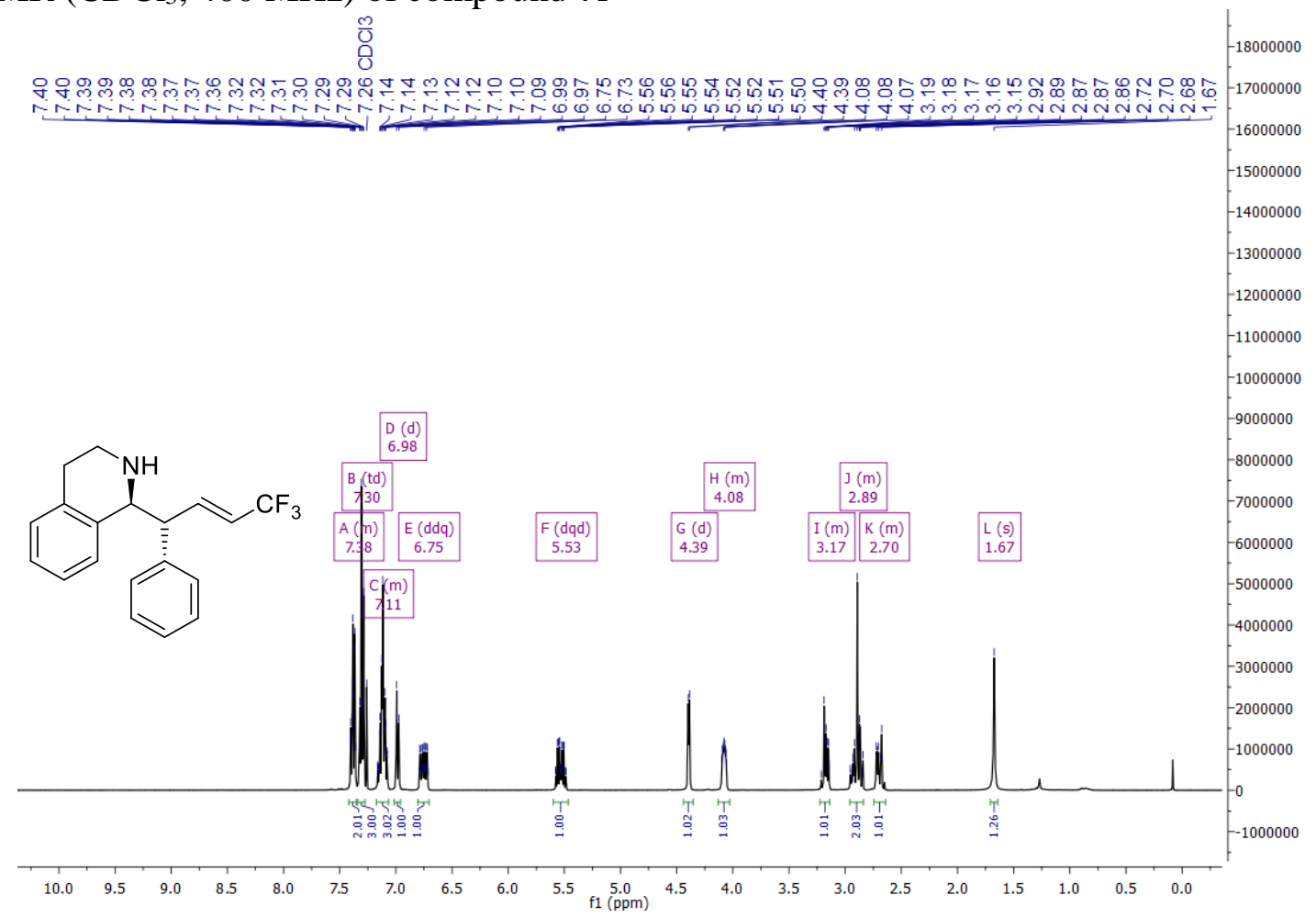

${ }^{13} \mathrm{C} \mathrm{NMR}\left(\mathrm{CDCl}_{3}, 101 \mathrm{MHz}\right)$ of compound $7 \mathrm{l}$

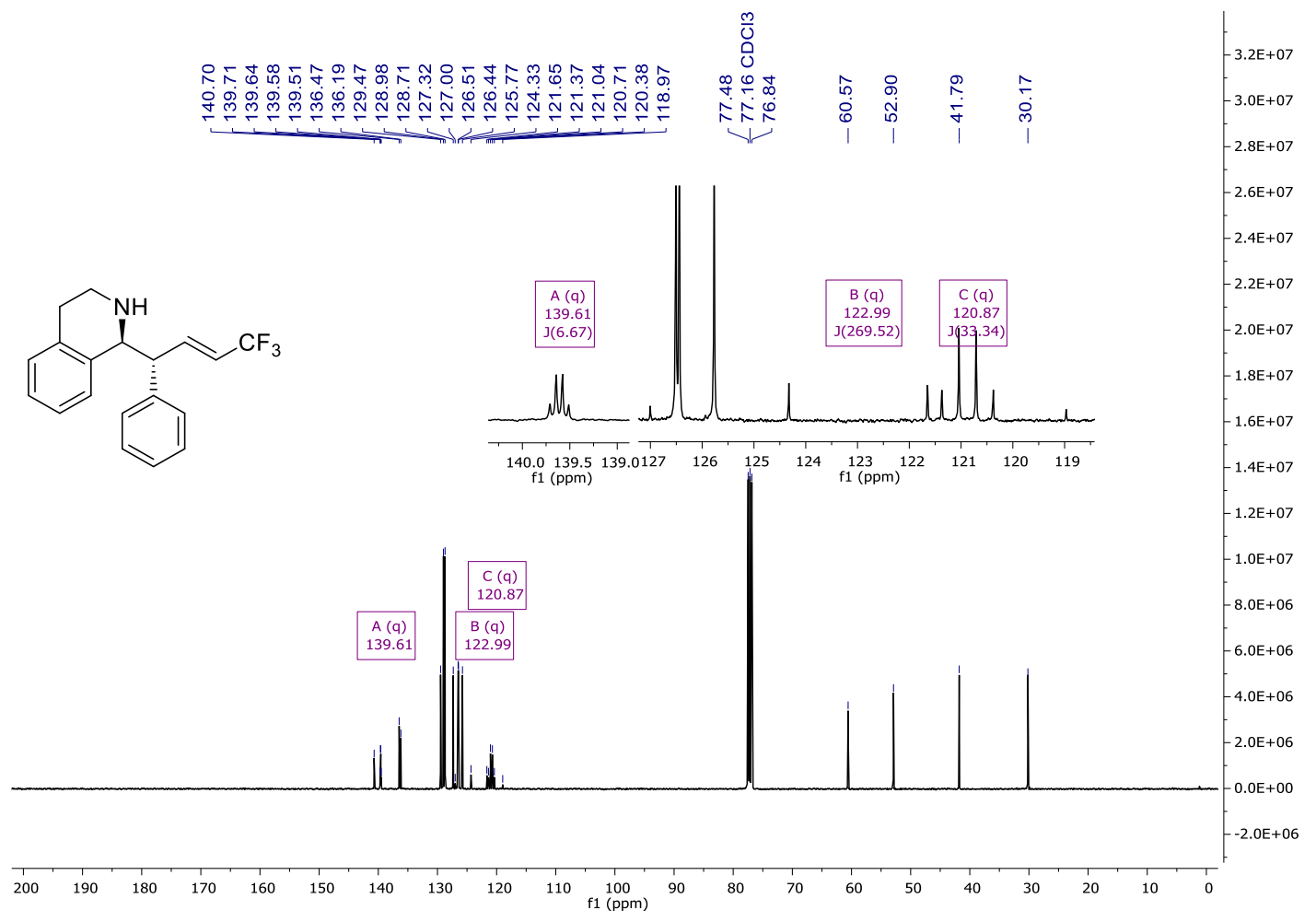


${ }^{19} \mathrm{~F} \mathrm{NMR}\left(\mathrm{CDCl}_{3}, 377 \mathrm{MHz}\right)$ of compound $7 \mathrm{l}$

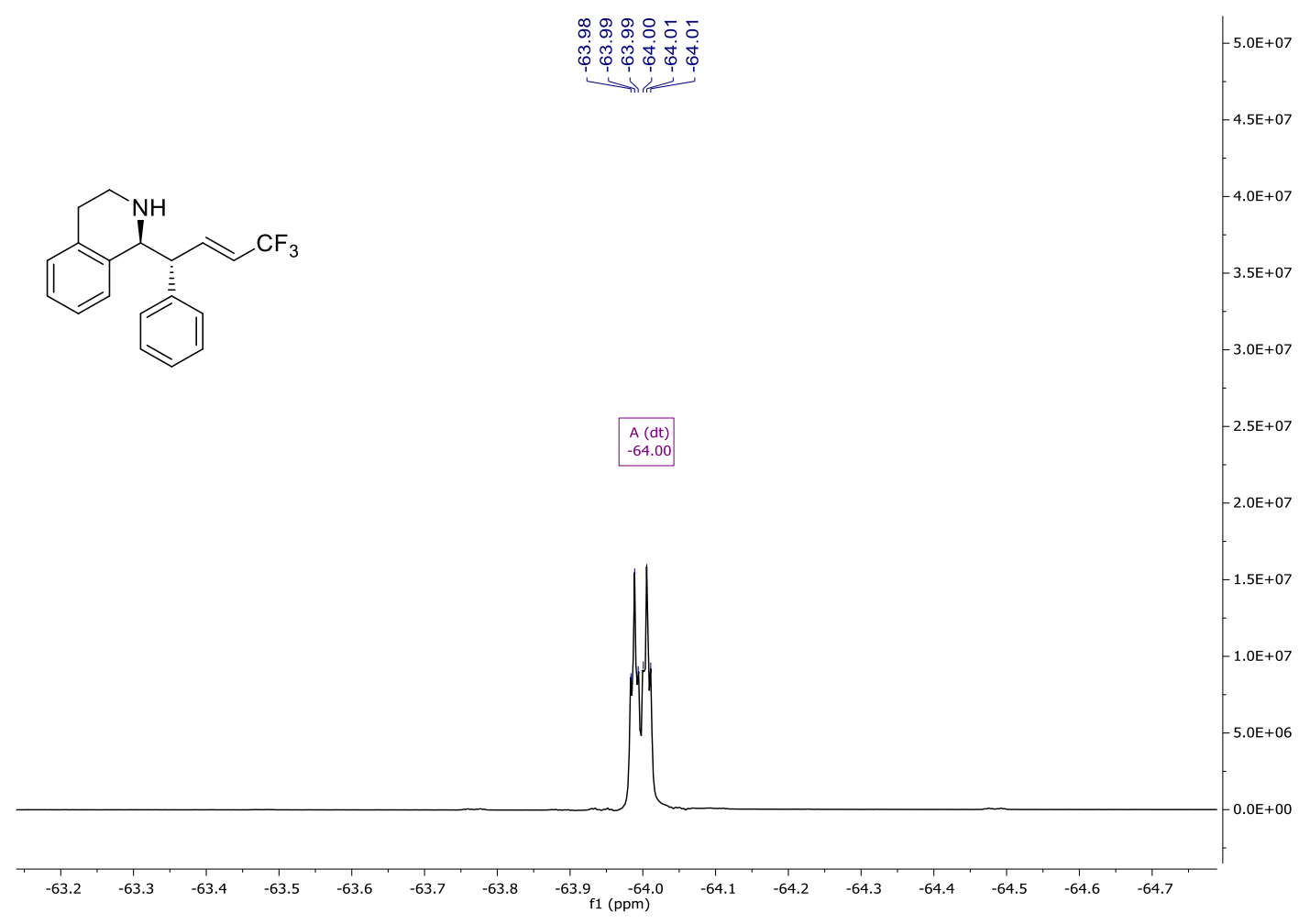

${ }^{1} \mathrm{H} \mathrm{NMR}\left(\mathrm{CDCl}_{3}, 400 \mathrm{MHz}\right)$ of compound $\mathbf{7 m}$

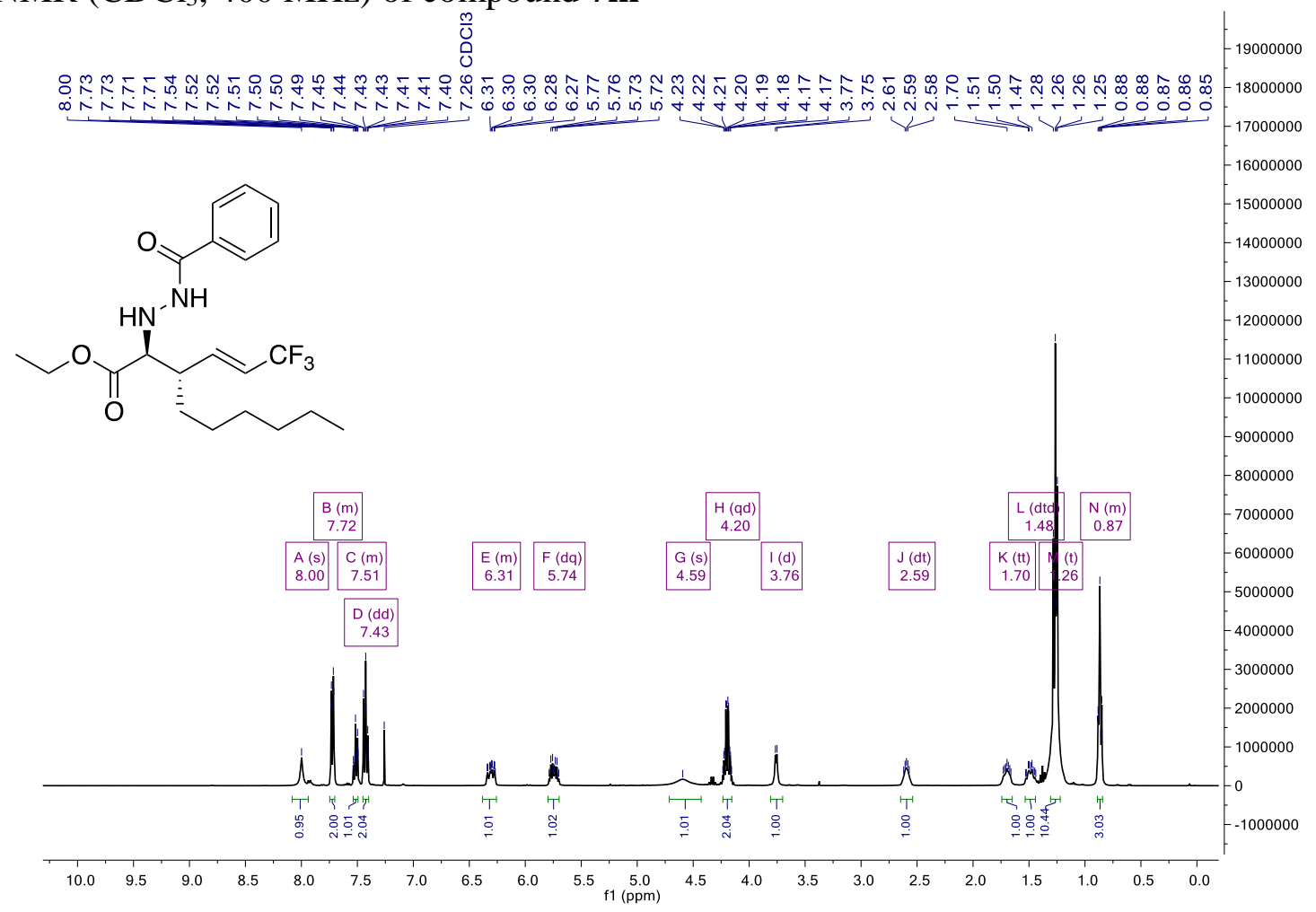


${ }^{13} \mathrm{C} \mathrm{NMR}\left(\mathrm{CDCl}_{3}, 101 \mathrm{MHz}\right)$ of compound $7 \mathbf{m}$

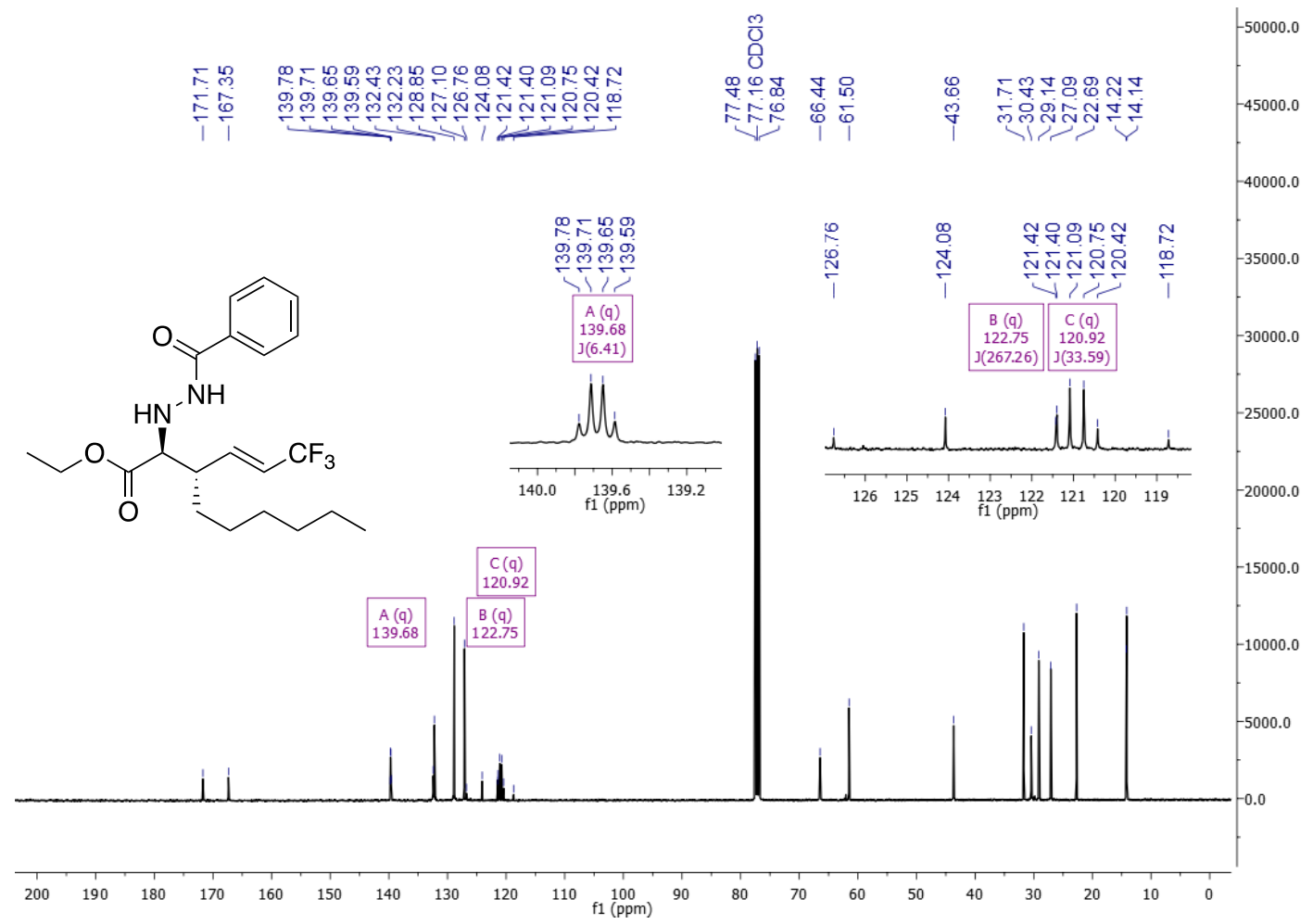

${ }^{19} \mathrm{~F} \mathrm{NMR}\left(\mathrm{CDCl}_{3}, 377 \mathrm{MHz}\right)$ of compound $7 \mathbf{m}$

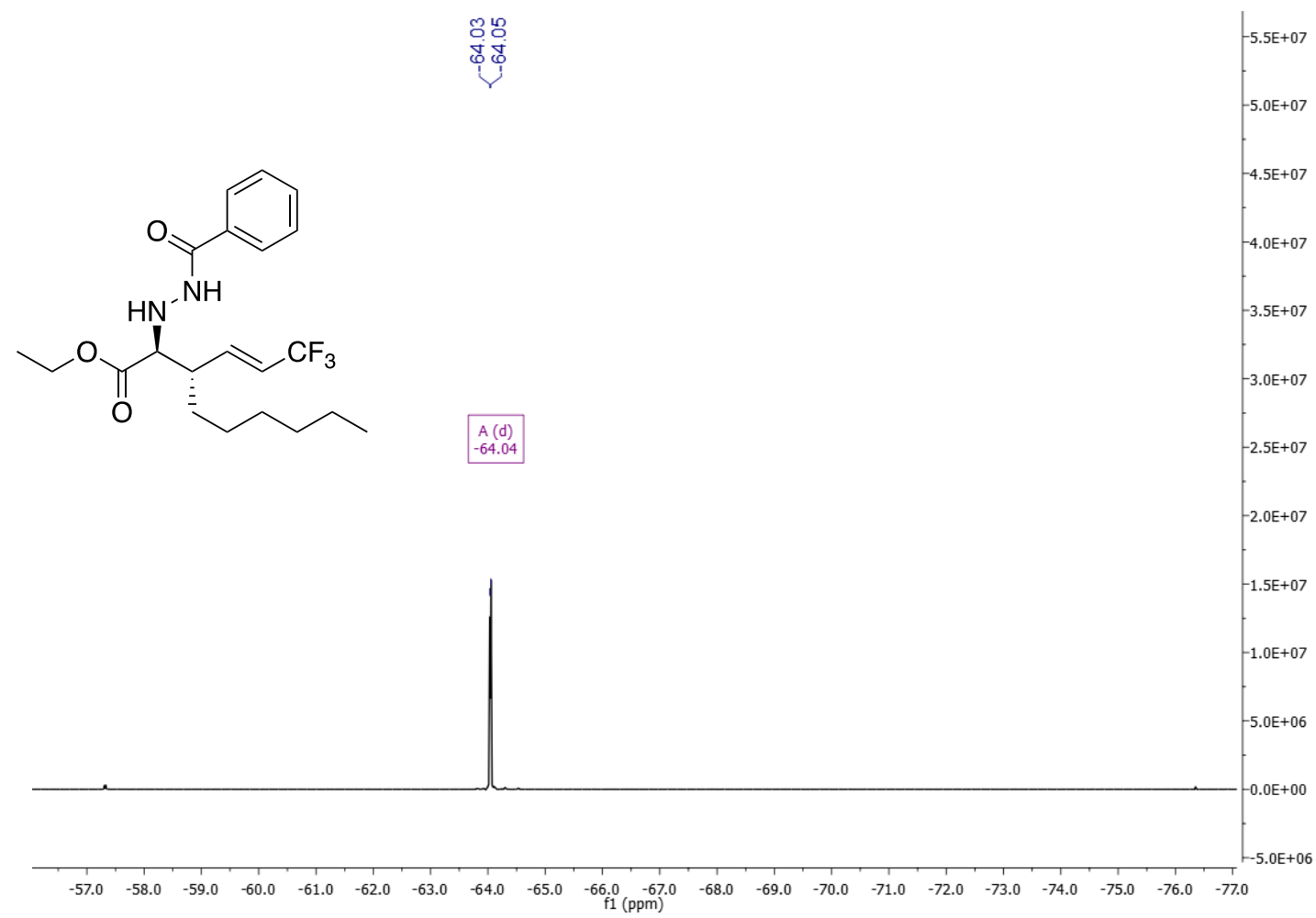


${ }^{1} \mathrm{H} \mathrm{NMR}\left(\mathrm{CDCl}_{3}, 400 \mathrm{MHz}\right)$ of compound $\mathbf{8 a}$

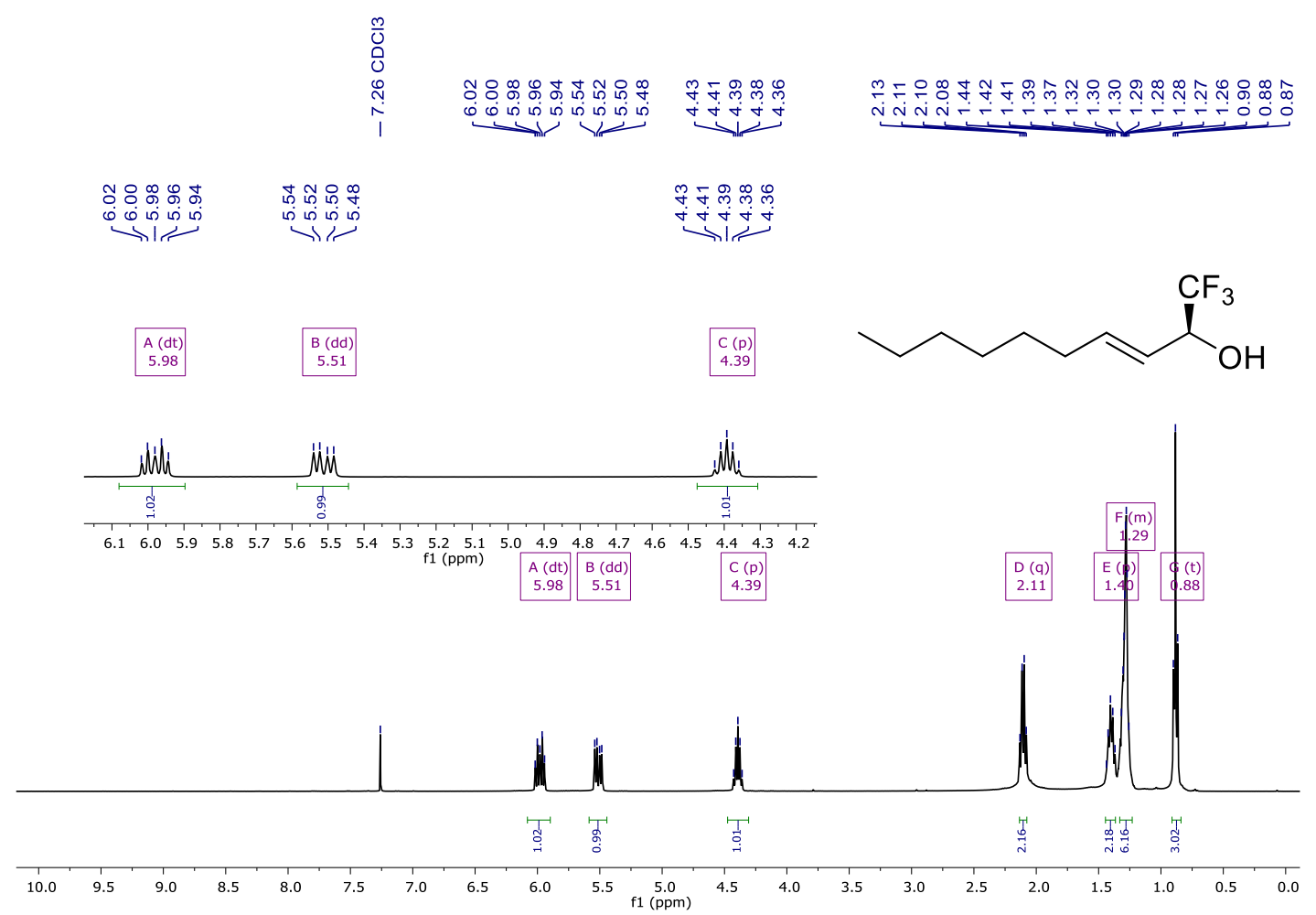

${ }^{13} \mathrm{C} \mathrm{NMR}\left(\mathrm{CDCl}_{3}, 101 \mathrm{MHz}\right)$ of compound $\mathbf{8 a}$

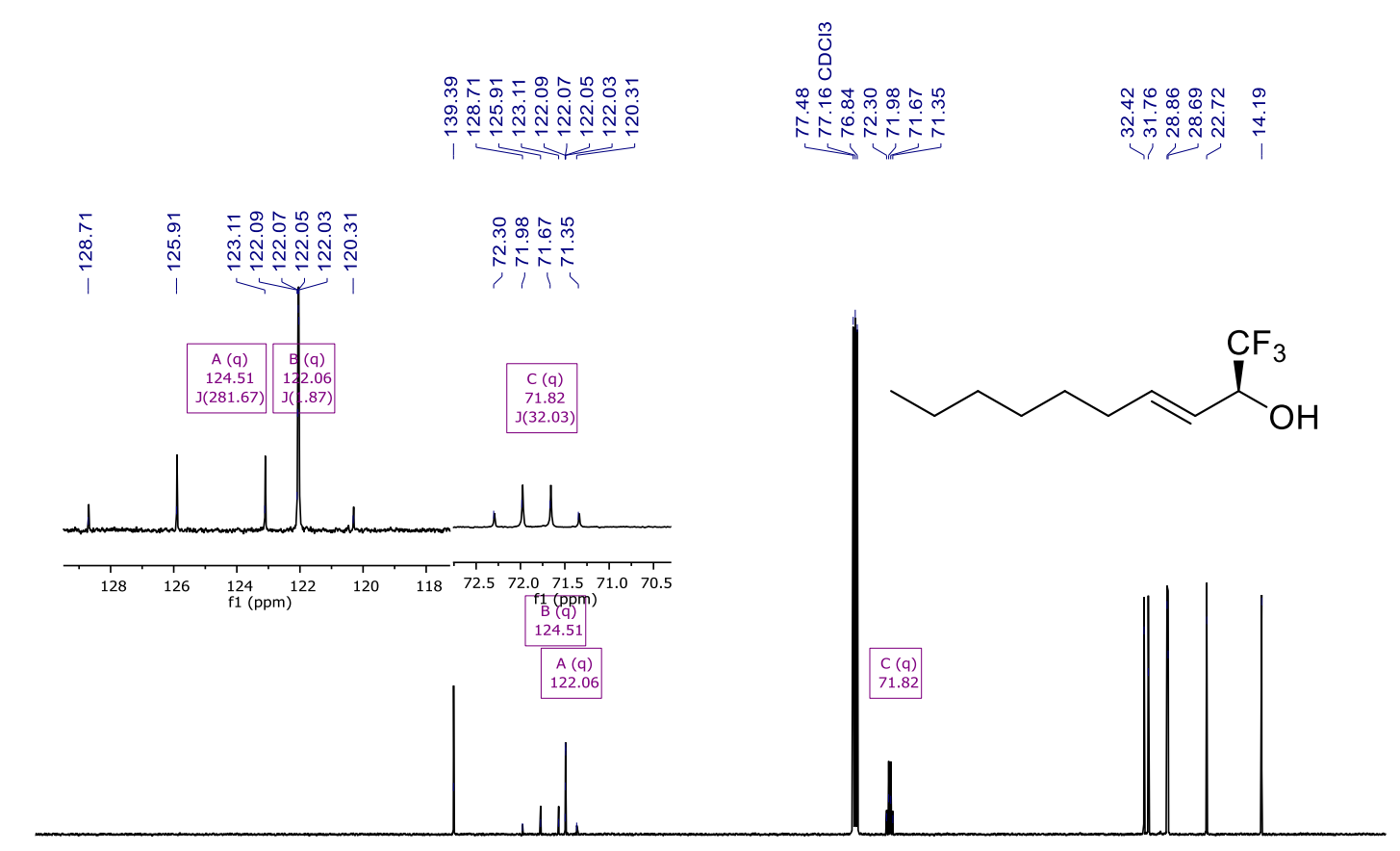

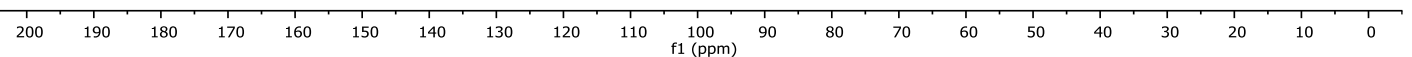


${ }^{19} \mathrm{~F}$ NMR $\left(\mathrm{CDCl}_{3}, 377 \mathrm{MHz}\right)$ of compound $8 \mathbf{a}$

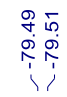

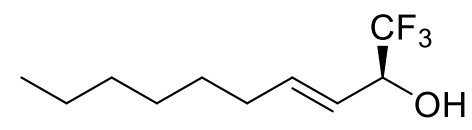

\section{${ }^{1} \mathrm{H}$ NMR $\left(\mathrm{CDCl}_{3}, 400 \mathrm{MHz}\right)$ of compound $\mathbf{8 b}$}

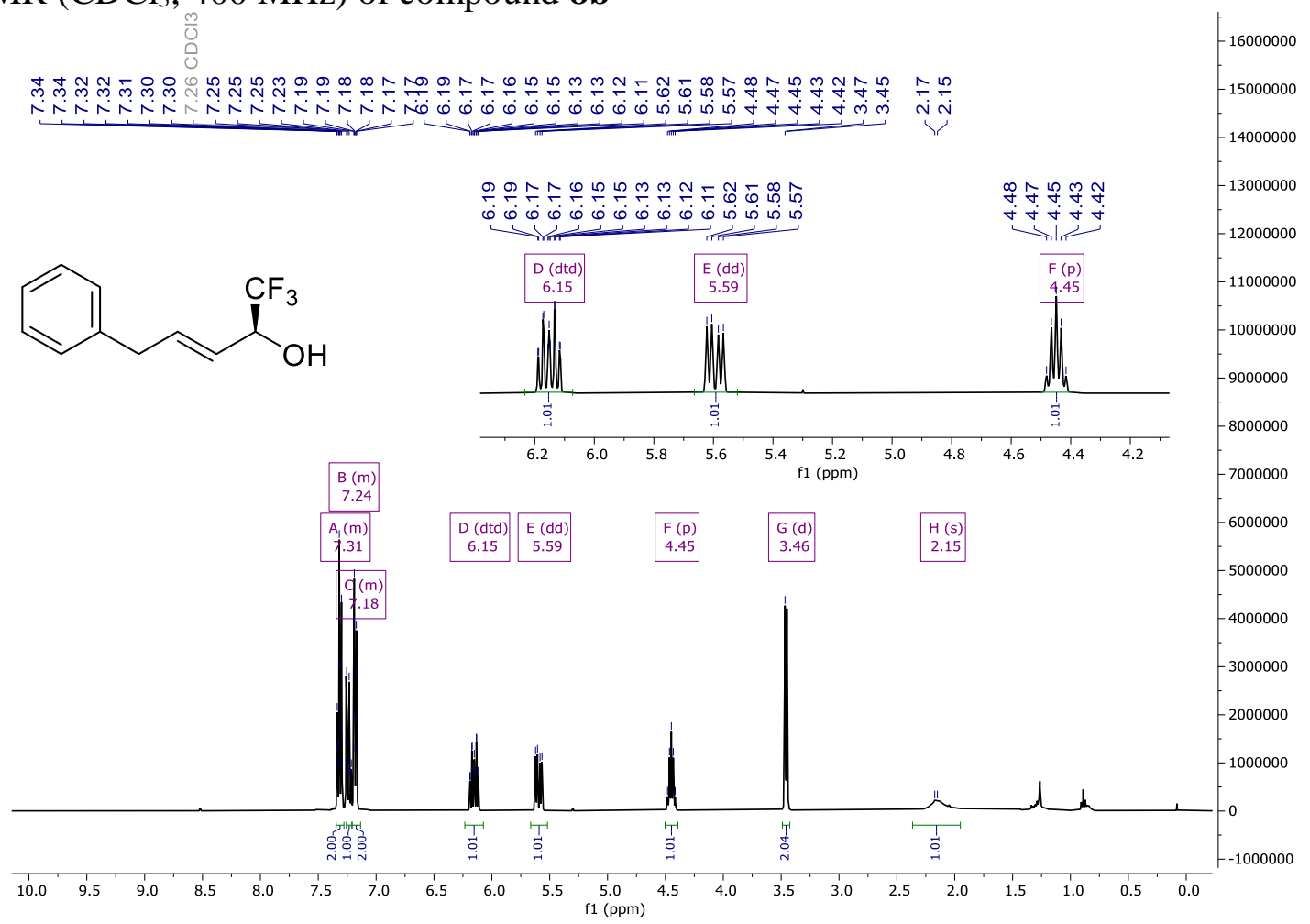


${ }^{13} \mathrm{C}$ NMR $\left(\mathrm{CDCl}_{3}, 101 \mathrm{MHz}\right)$ of compound $\mathbf{8 b}$

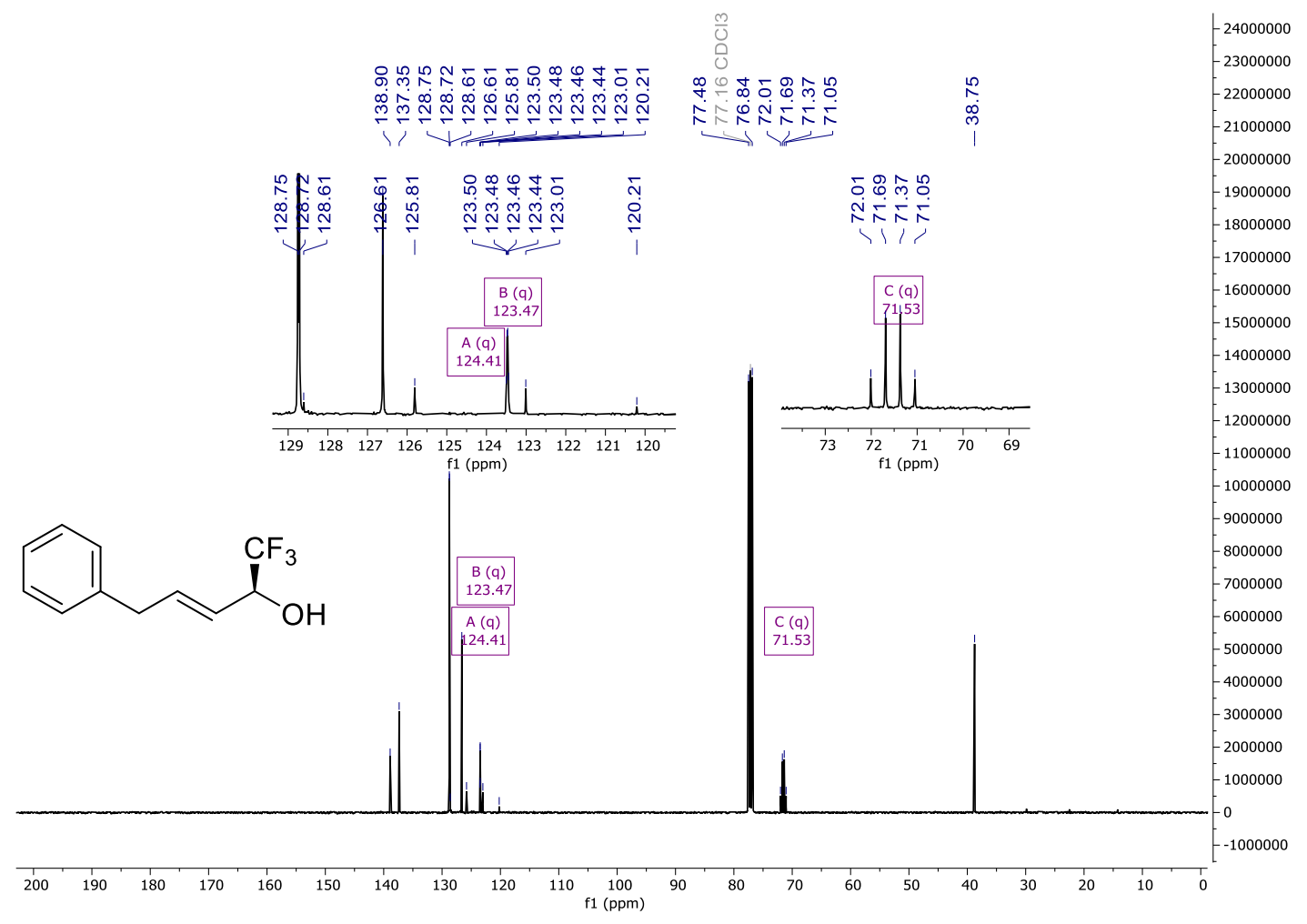

${ }^{19} \mathrm{~F}$ NMR $\left(\mathrm{CDCl}_{3}, 377 \mathrm{MHz}\right)$ of compound $\mathbf{8 b}$

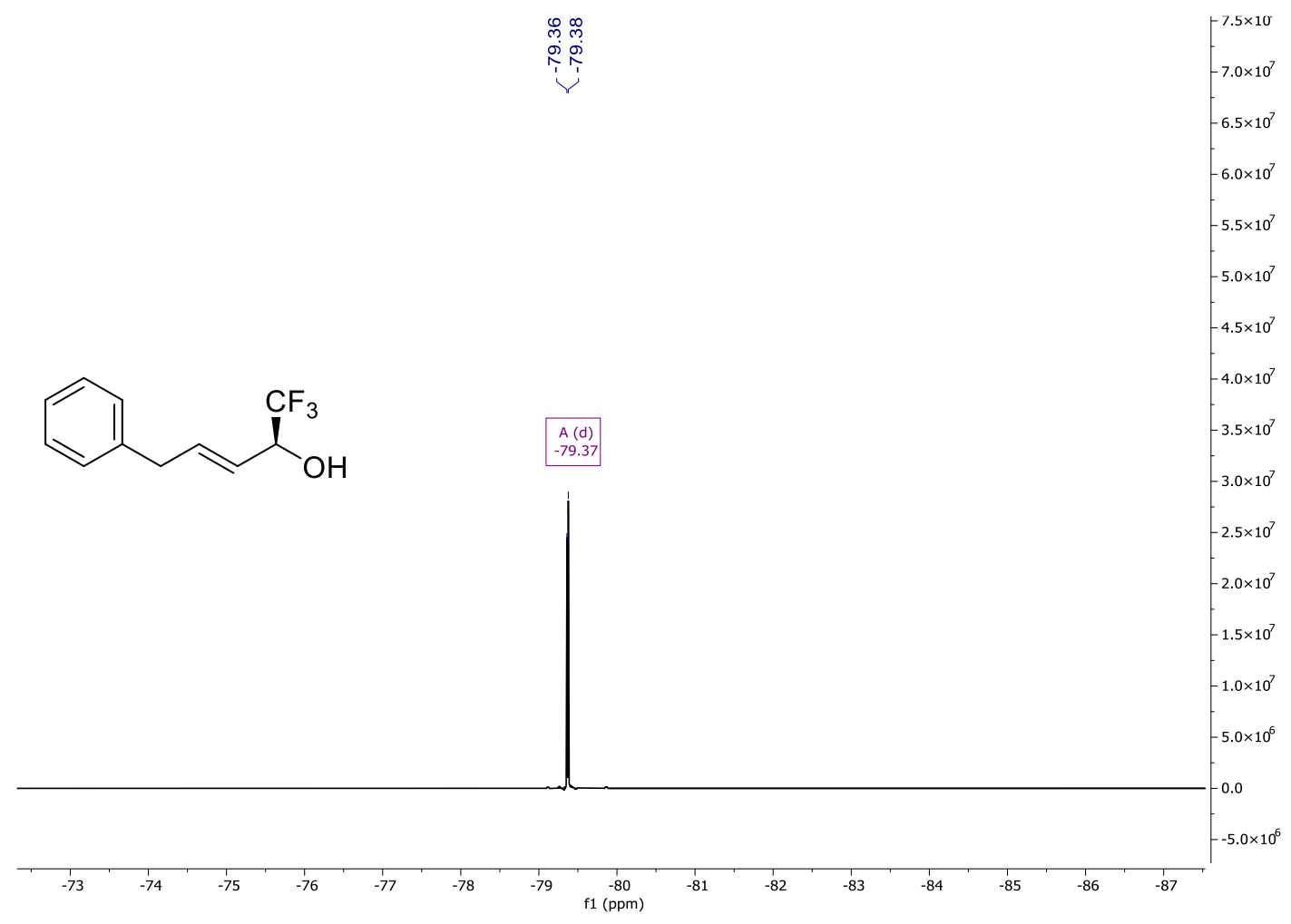


${ }^{1} \mathrm{H}$ NMR $\left(\mathrm{CDCl}_{3}, 400 \mathrm{MHz}\right)$ of compound $\mathbf{8 c}$

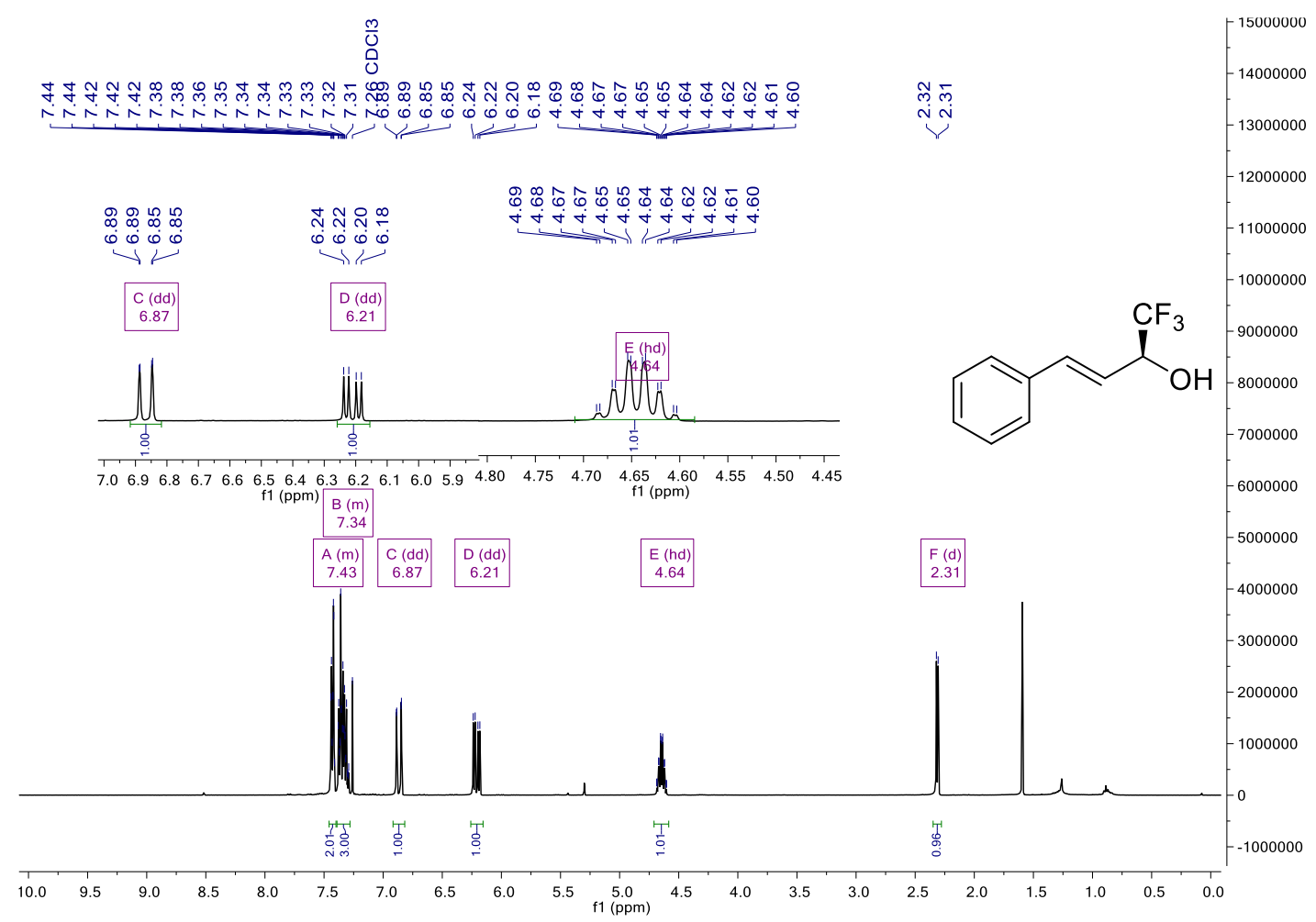

${ }^{13} \mathrm{C}$ NMR $\left(\mathrm{CDCl}_{3}, 101 \mathrm{MHz}\right)$ of compound $8 \mathrm{c}$

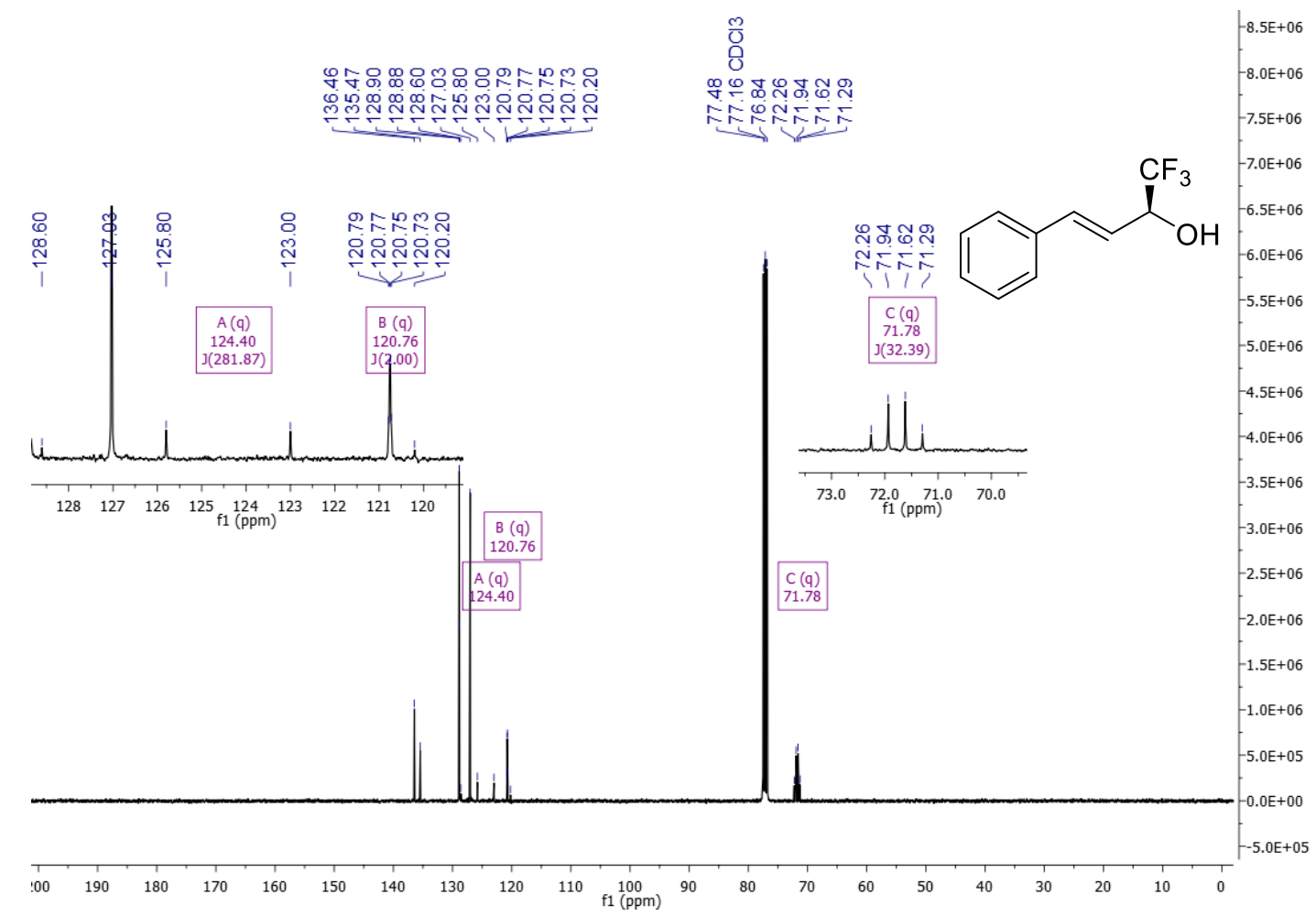


${ }^{19} \mathrm{~F}$ NMR $\left(\mathrm{CDCl}_{3}, 377 \mathrm{MHz}\right)$ of compound $\mathbf{8 c}$

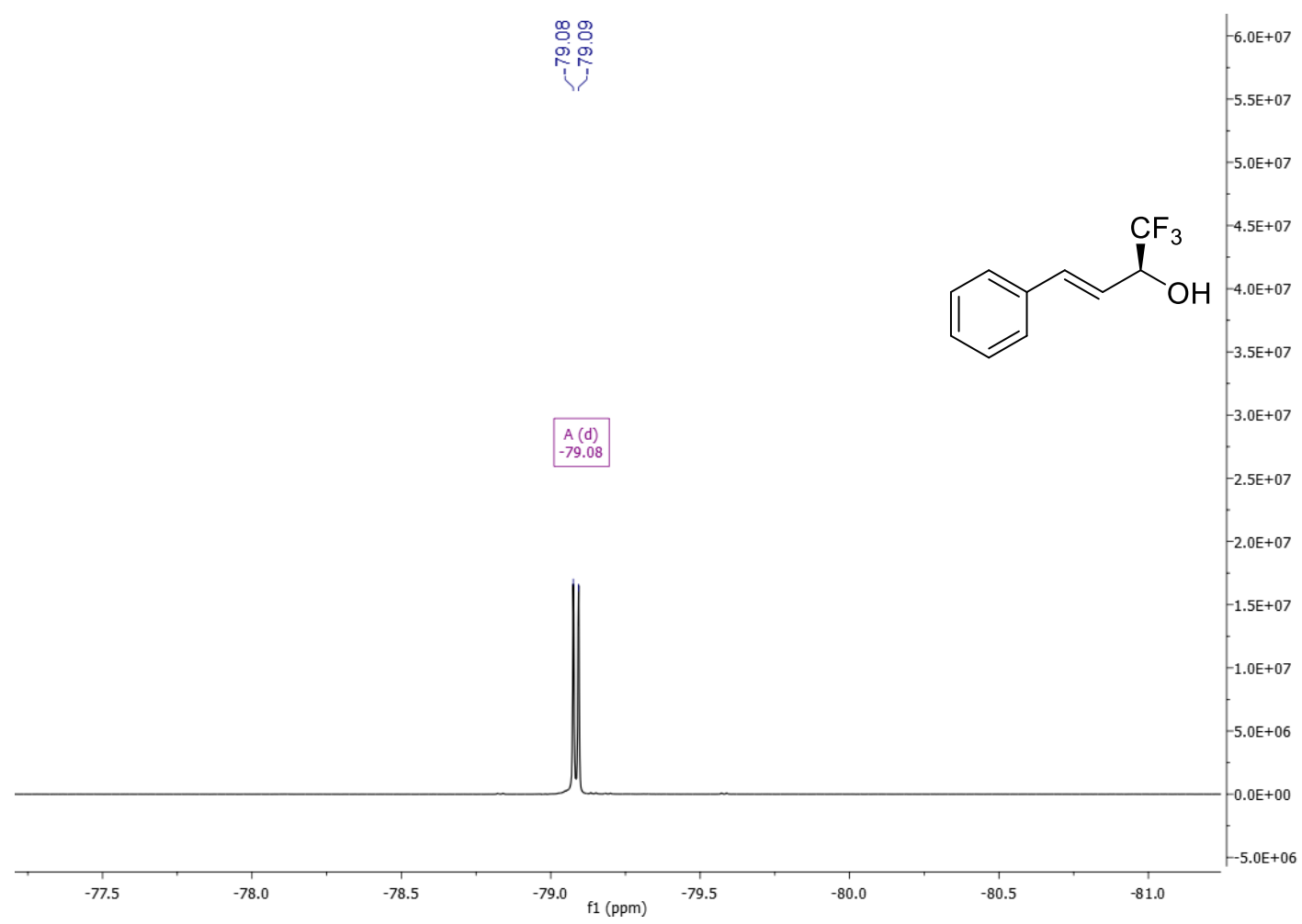

${ }^{1} \mathrm{H} \mathrm{NMR}\left(\mathrm{CDCl}_{3}, 400 \mathrm{MHz}\right)$ of compound 9

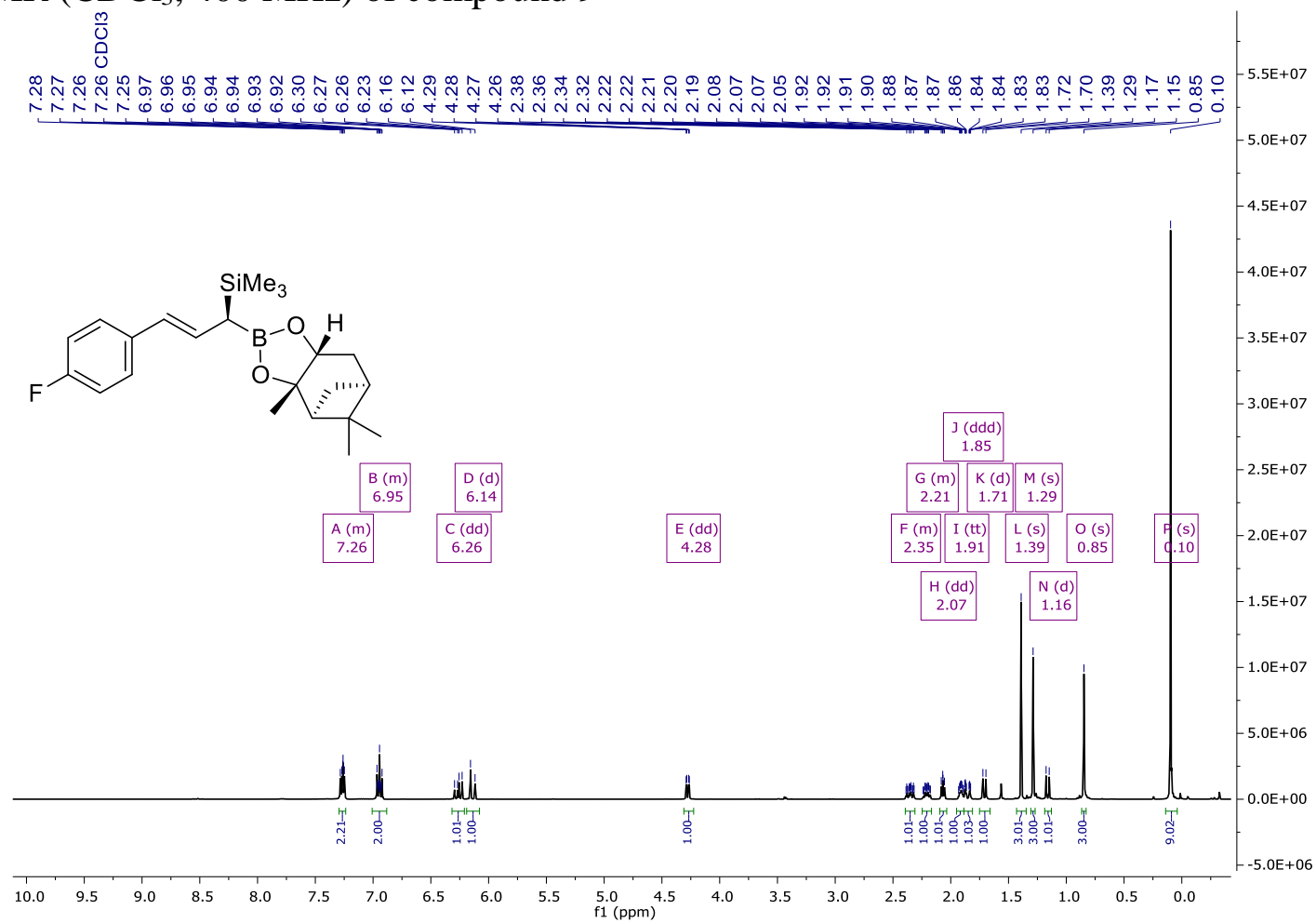


${ }^{13} \mathrm{C} \mathrm{NMR}\left(\mathrm{CDCl}_{3}, 101 \mathrm{MHz}\right)$ of compound 9

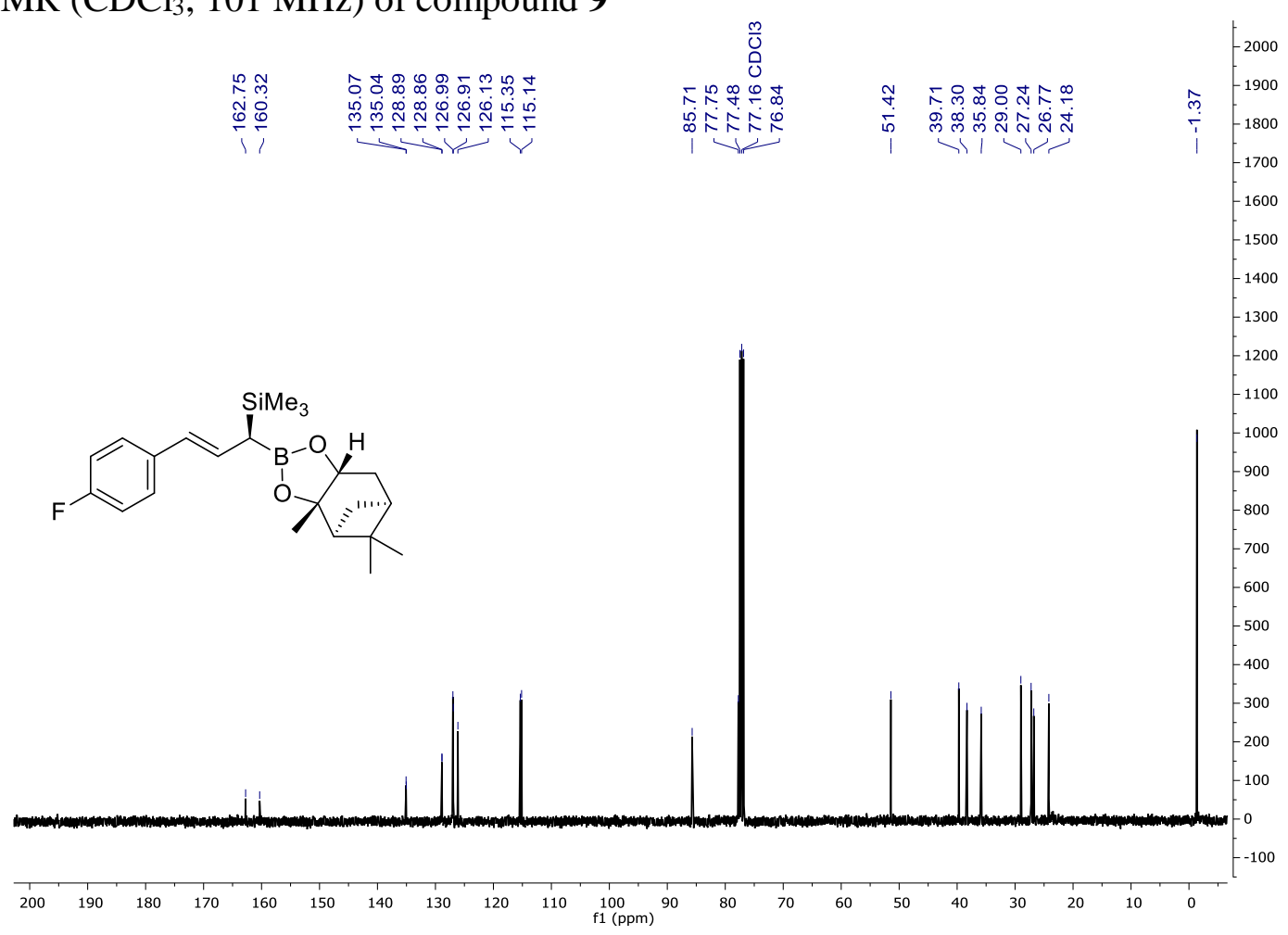

${ }^{19} \mathrm{~F} \mathrm{NMR}\left(\mathrm{CDCl}_{3}, 377 \mathrm{MHz}\right)$ of compound 9

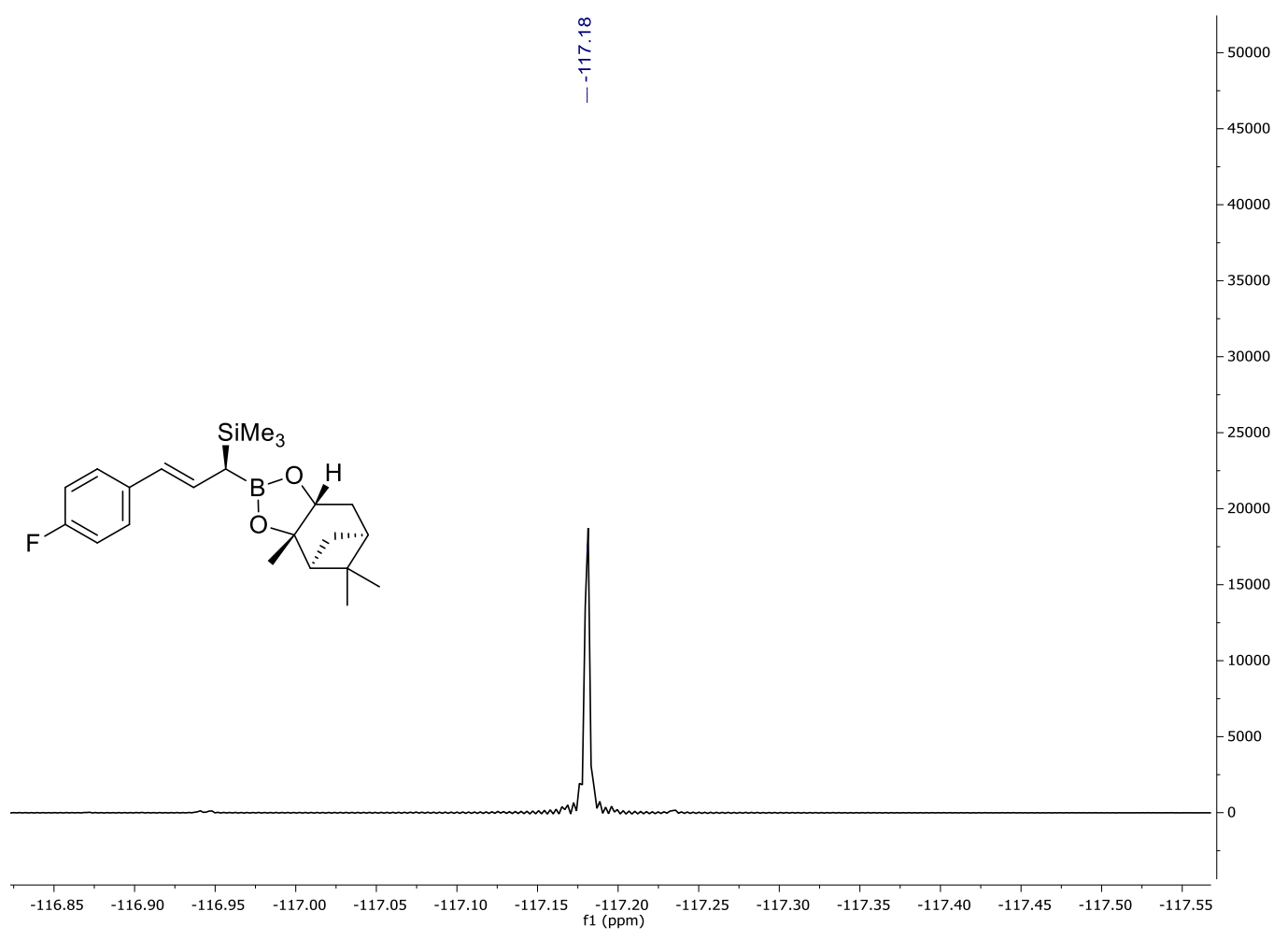


${ }^{11} \mathrm{~B}$ NMR $\left(\mathrm{CDCl}_{3}, 128 \mathrm{MHz}\right)$ of compound 9

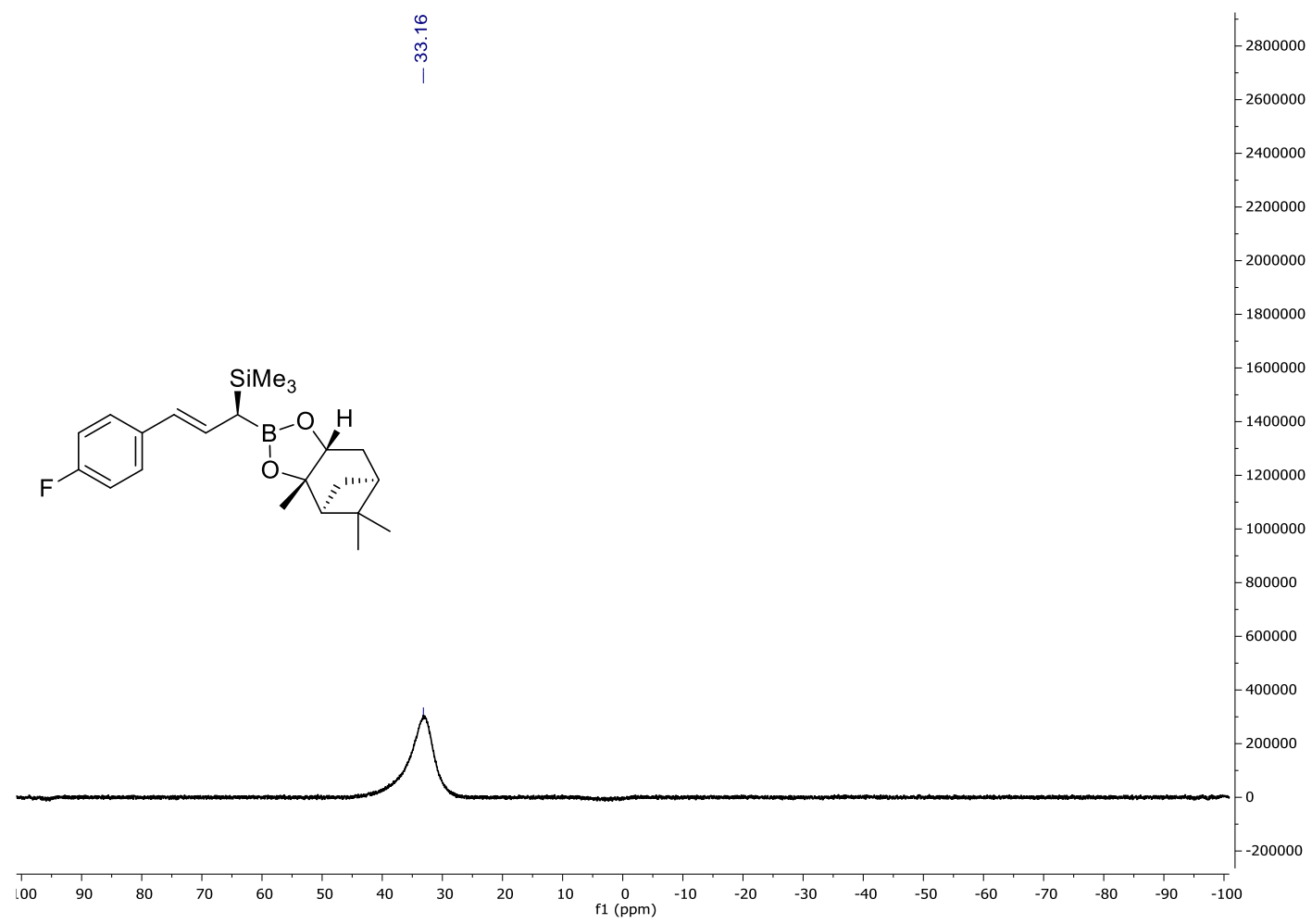

\section{${ }^{1} \mathrm{H} \mathrm{NMR}\left(\mathrm{CDCl}_{3}, 400 \mathrm{MHz}\right)$ of compound 10}

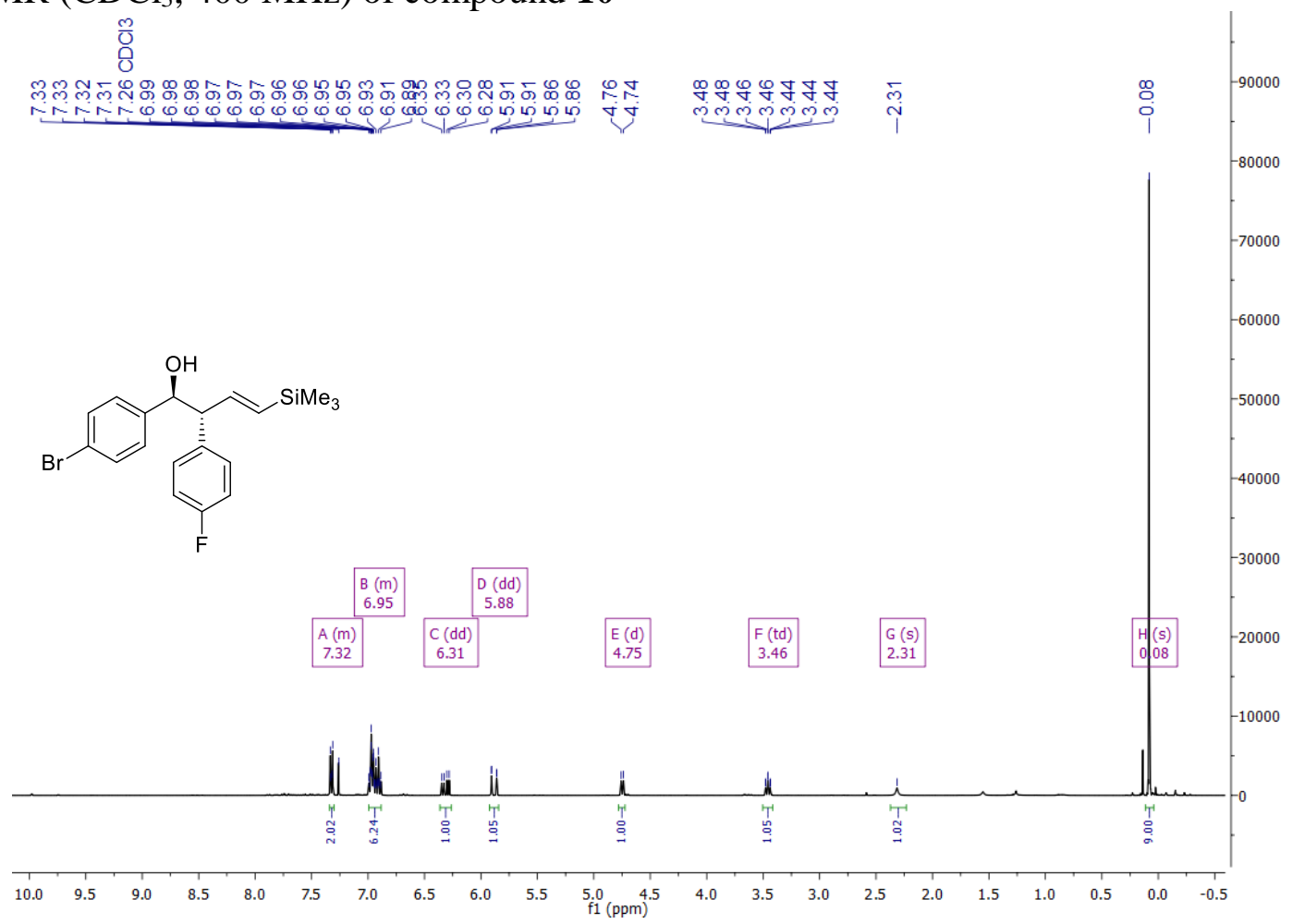


${ }^{13} \mathrm{C}$ NMR $\left(\mathrm{CDCl}_{3}, 101 \mathrm{MHz}\right)$ of compound $\mathbf{1 0}$

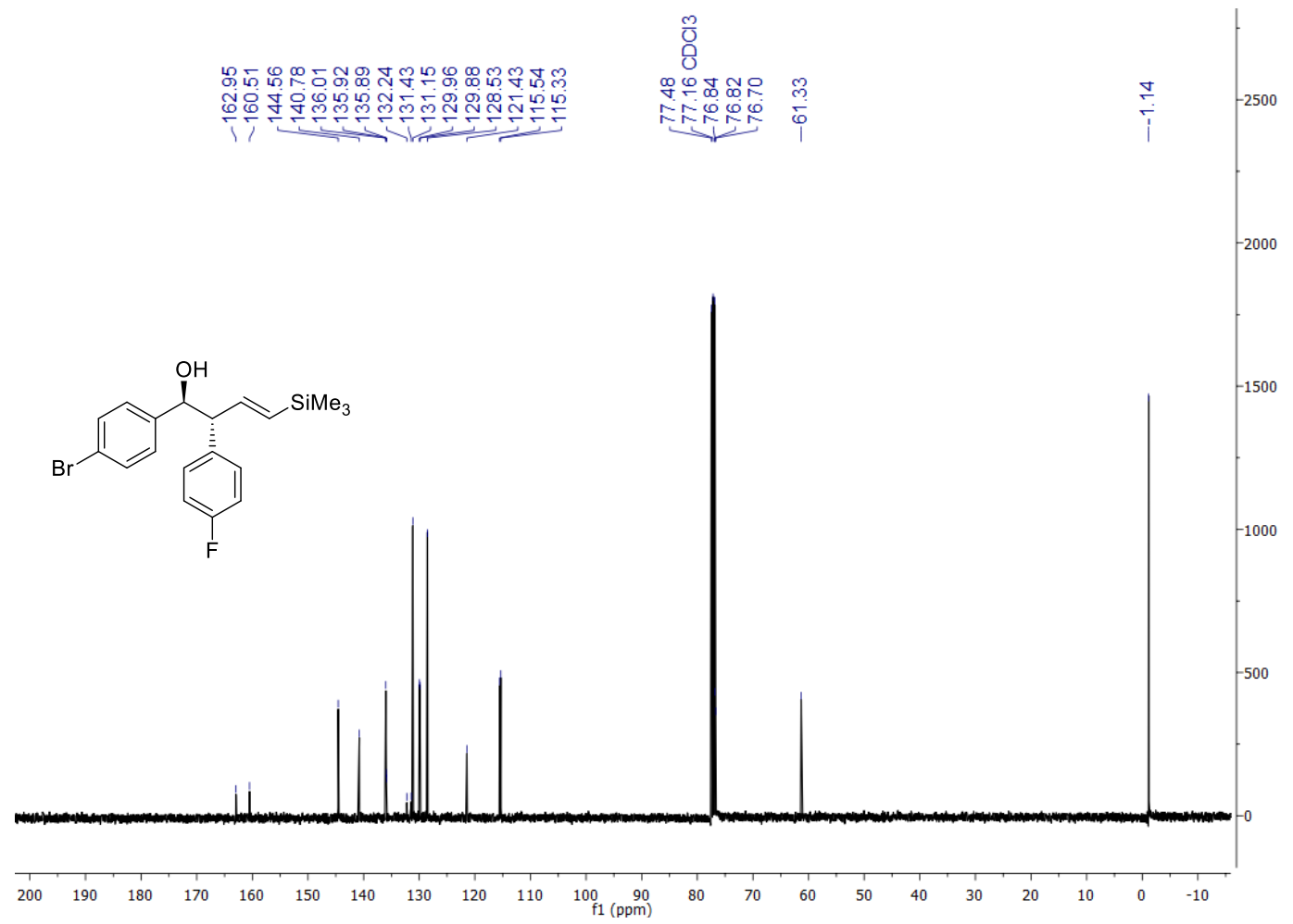

${ }^{19} \mathrm{~F}$ NMR $\left(\mathrm{CDCl}_{3}, 377 \mathrm{MHz}\right)$ of compound $\mathbf{1 0}$

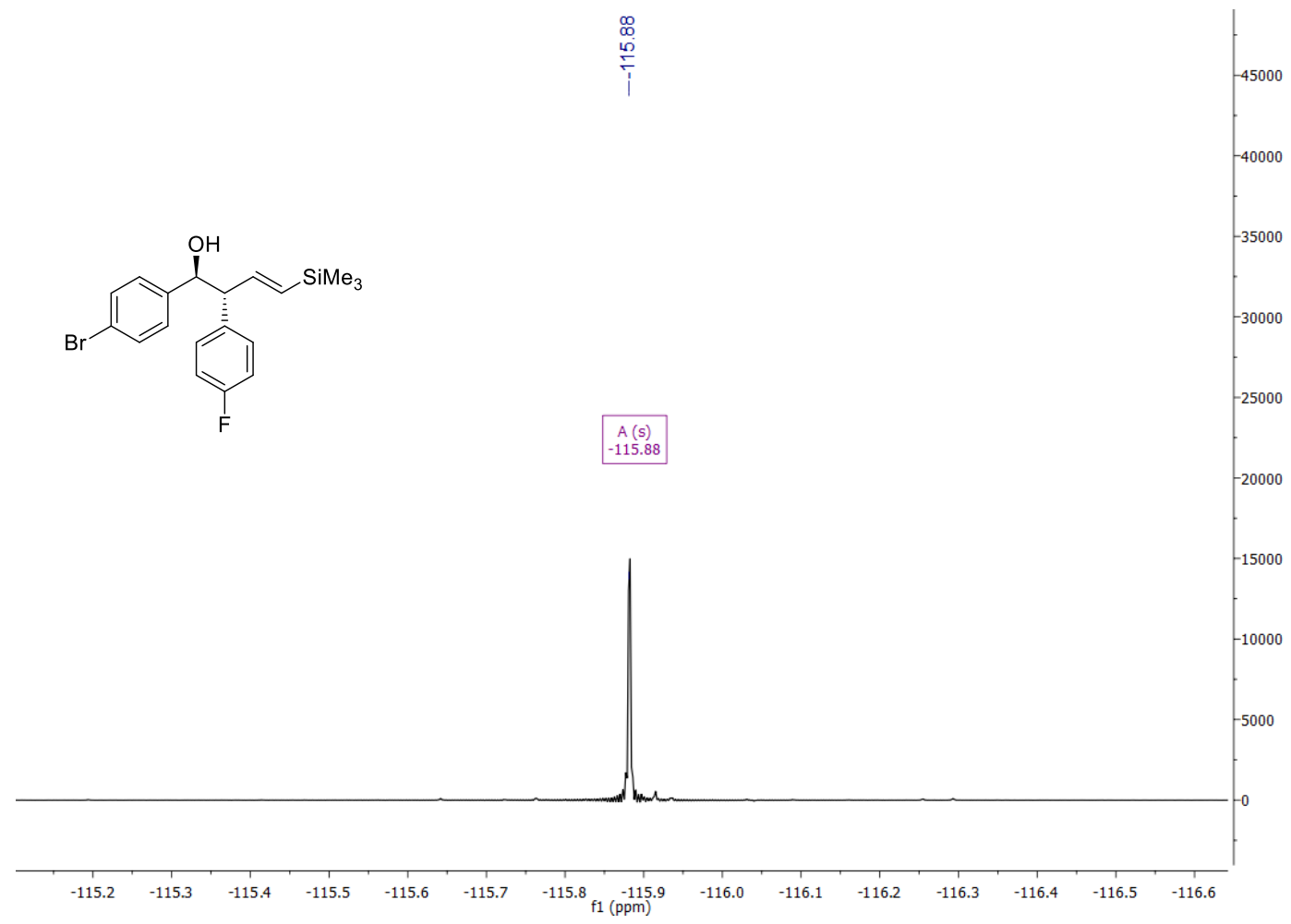


${ }^{1} \mathrm{H}$ NMR (Toluene- $d 8,400 \mathrm{MHz}$ ) of compound 2a

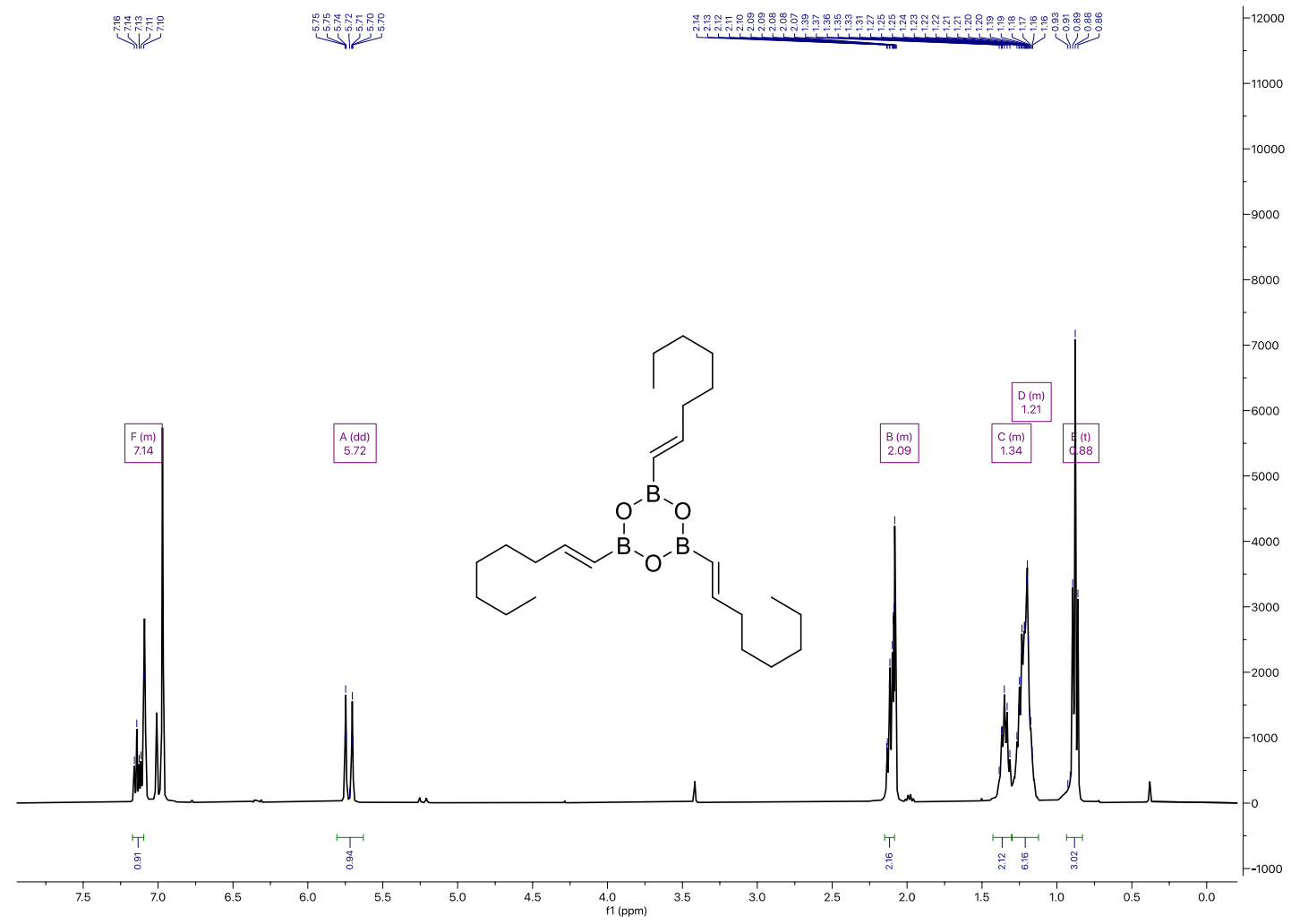

${ }^{11} \mathrm{~B}$ NMR (Toluene- $d 8,128 \mathrm{MHz}$ ) of compound $\mathbf{2 a}$

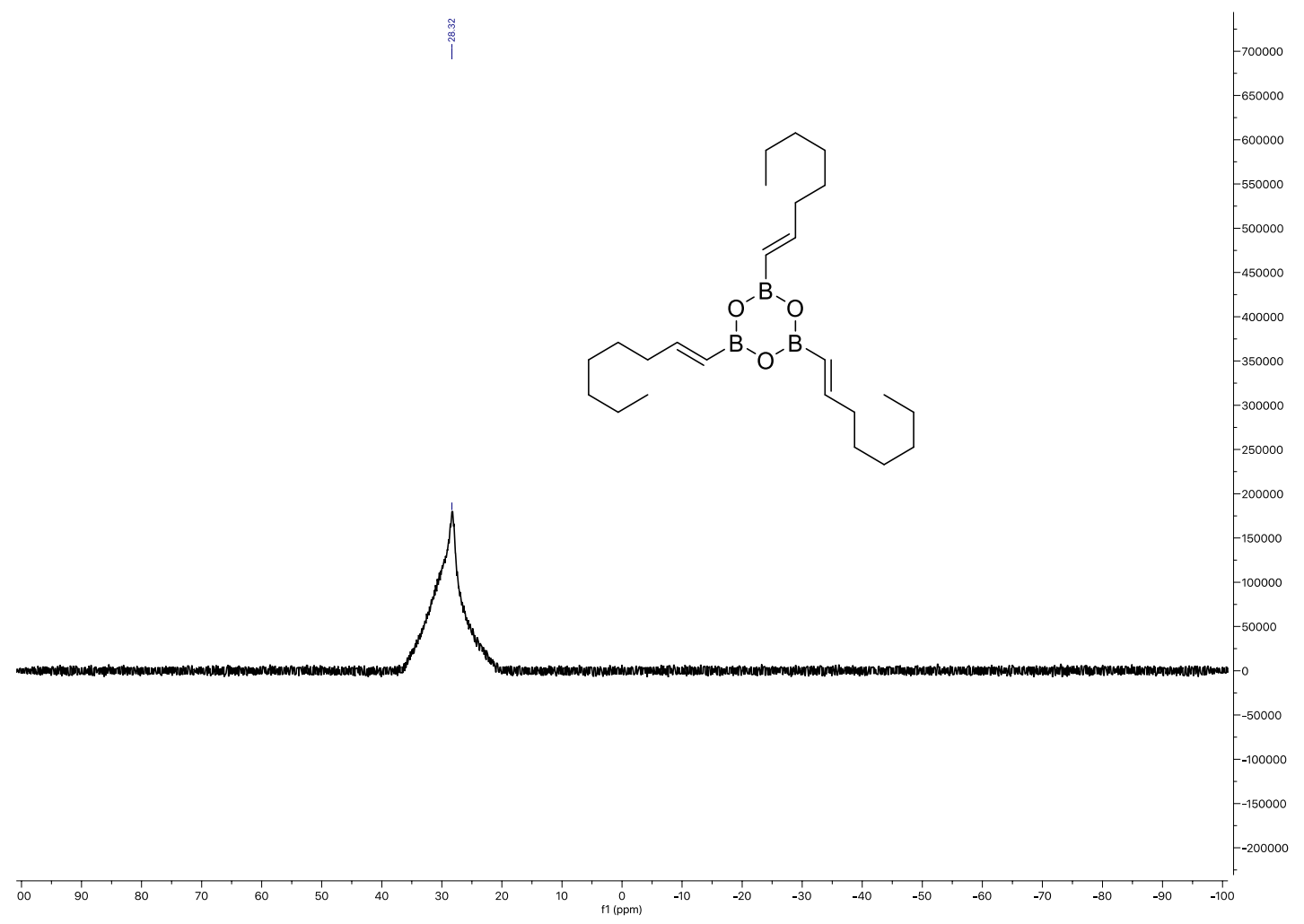


${ }^{1} \mathrm{H}$ NMR (Toluene- $d 8,400 \mathrm{MHz}$ ) of compound $\mathbf{2 b}$

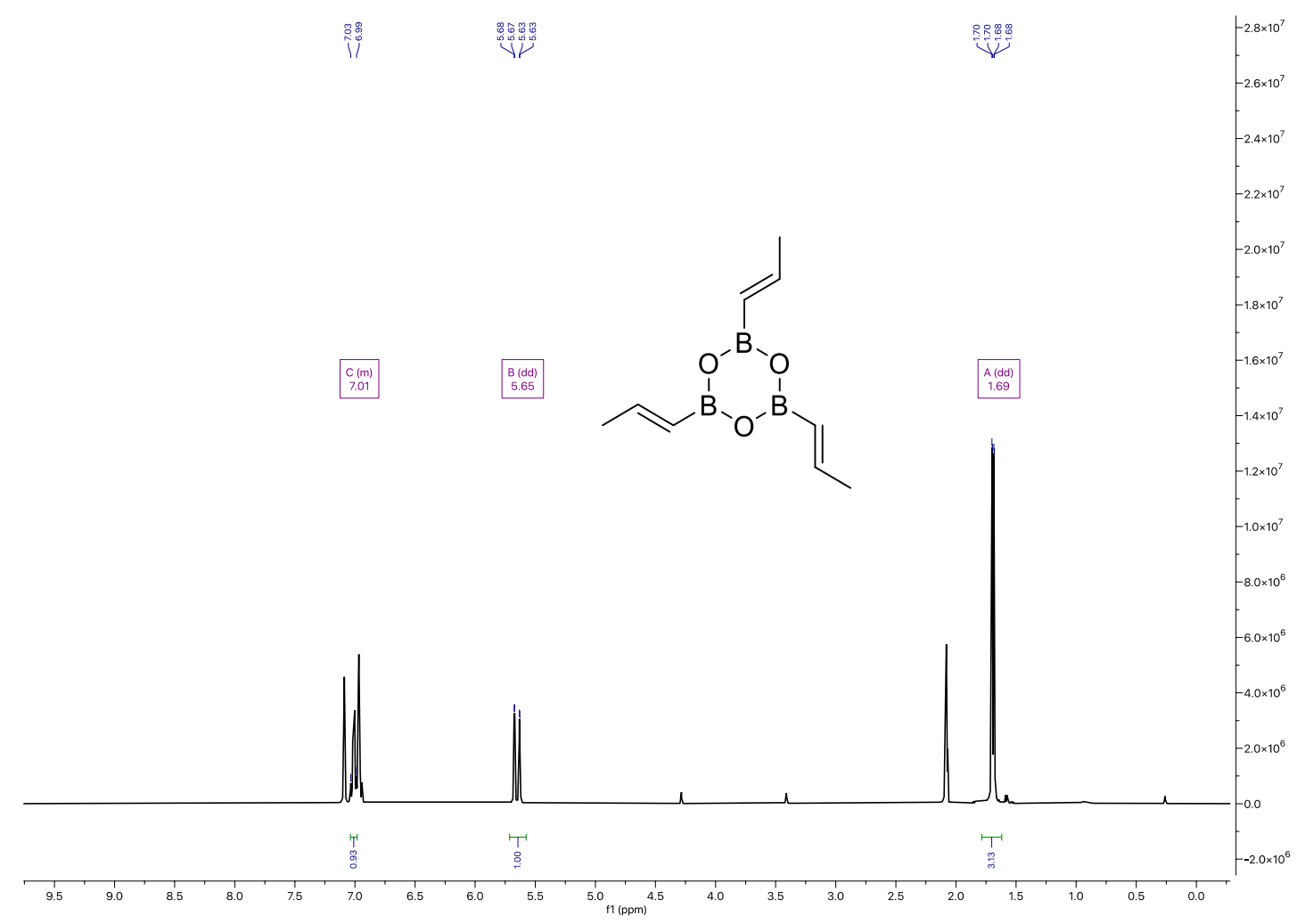

${ }^{11}$ B NMR (Toluene- $d 8,128 \mathrm{MHz}$ ) of compound $\mathbf{2 b}$

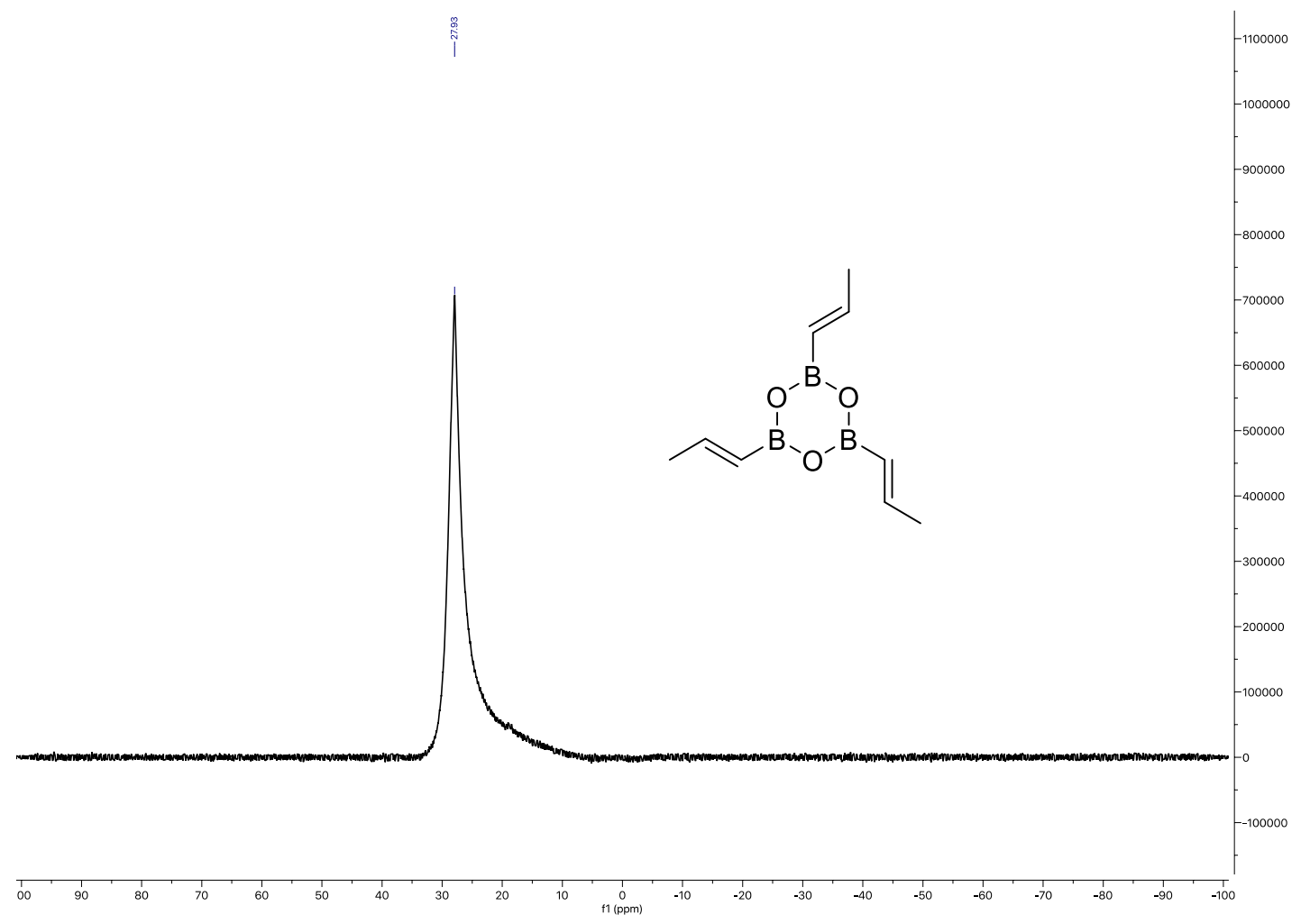


${ }^{1} \mathrm{H}$ NMR (Toluene- $d 8,400 \mathrm{MHz}$ ) of compound 2c

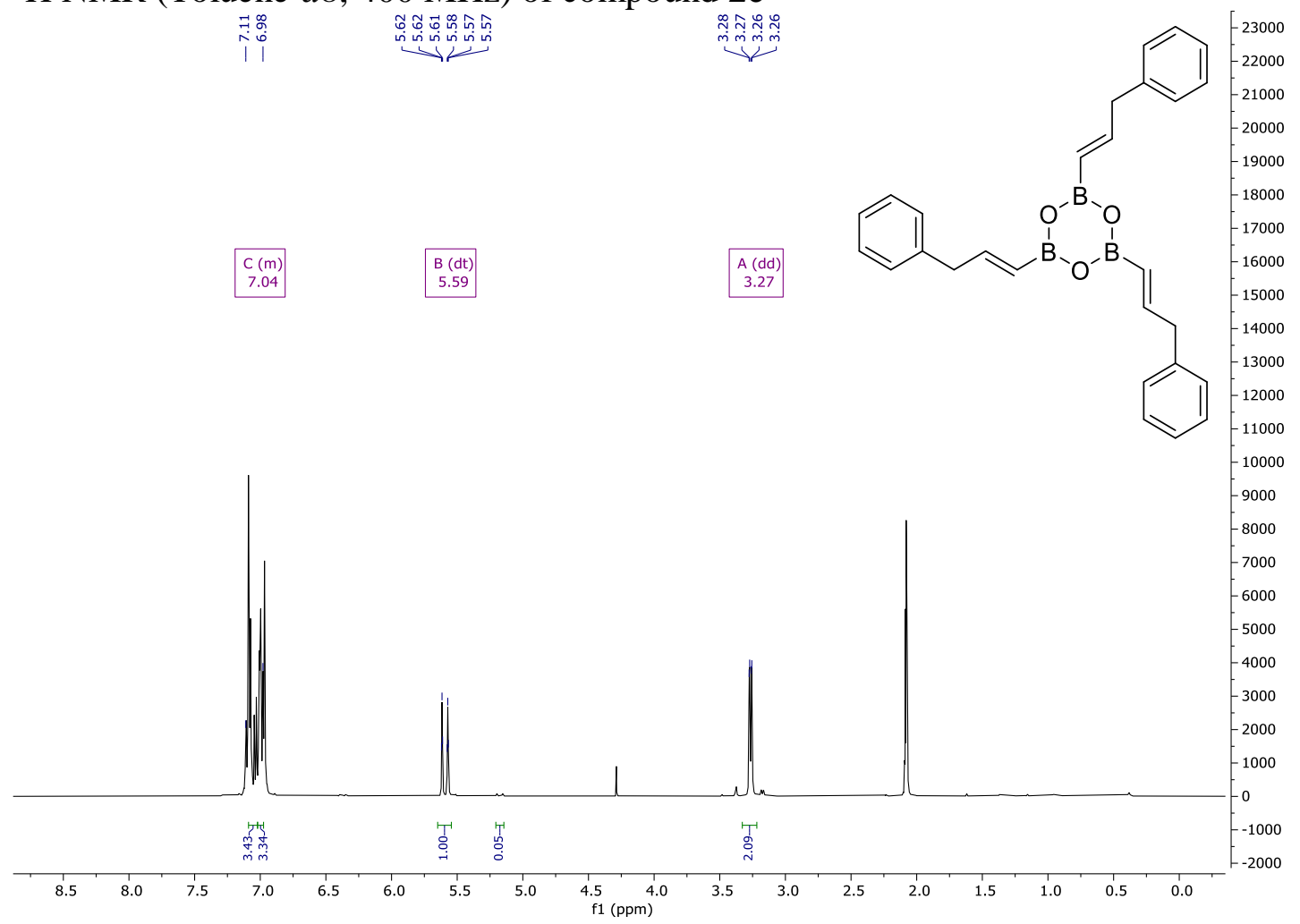

${ }^{11}$ B NMR (Toluene-d8, $128 \mathrm{MHz}$ ) of compound 2c

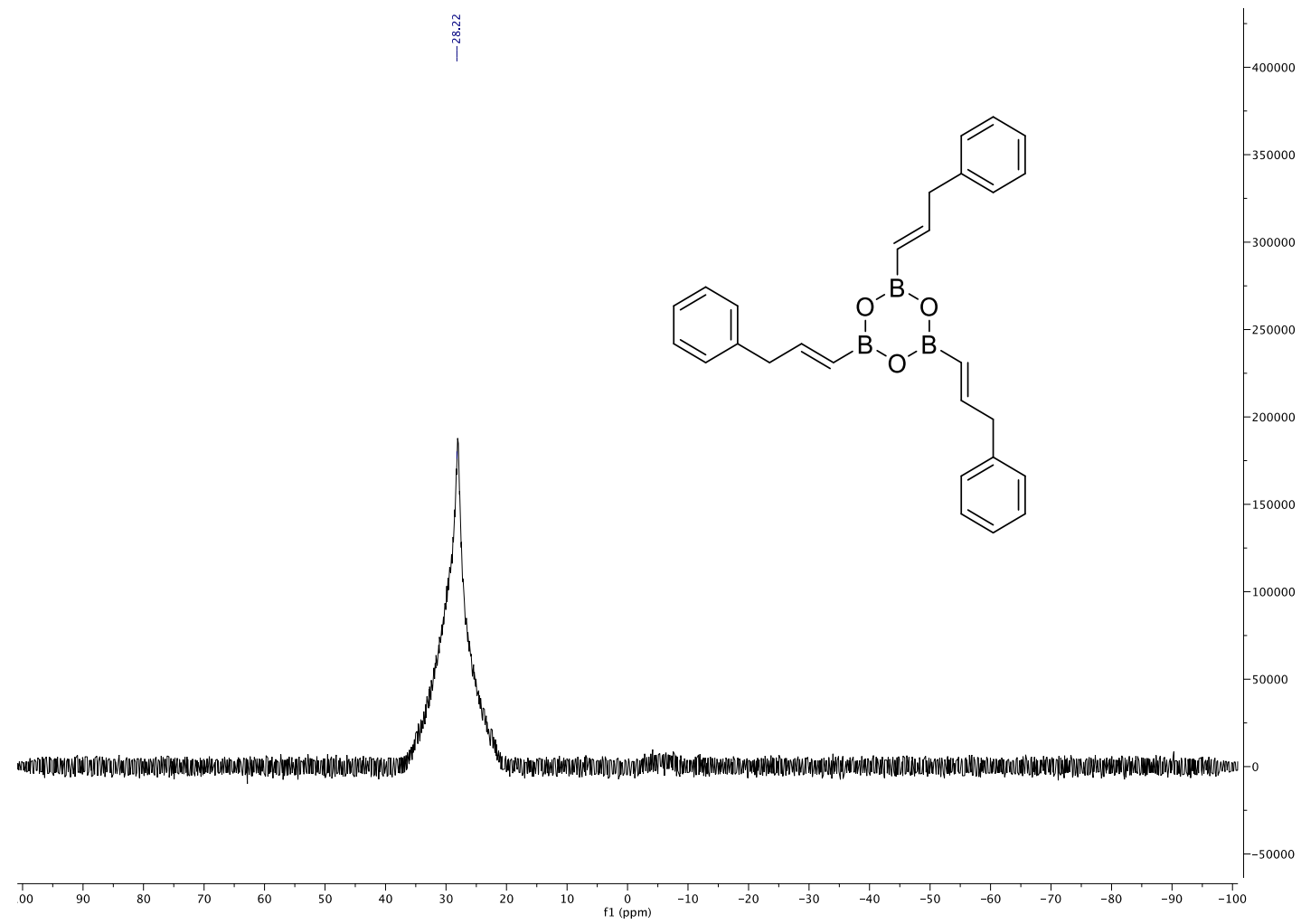


${ }^{1} \mathrm{H}$ NMR (Toluene- $d 8,400 \mathrm{MHz}$ ) of compound 2d

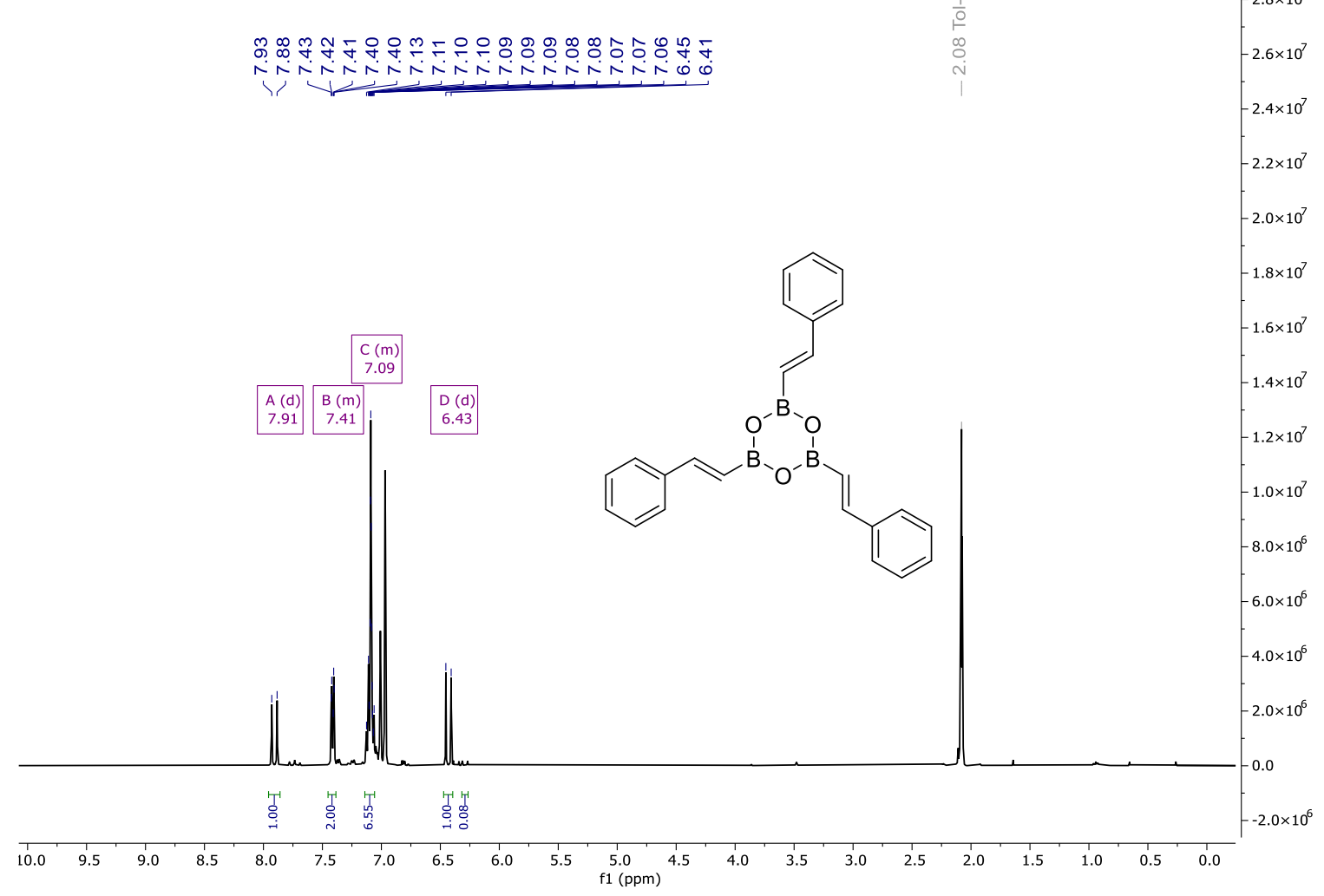

${ }^{11}$ B NMR (Toluene- $d 8,128 \mathrm{MHz}$ ) of compound $\mathbf{2 d}$ 


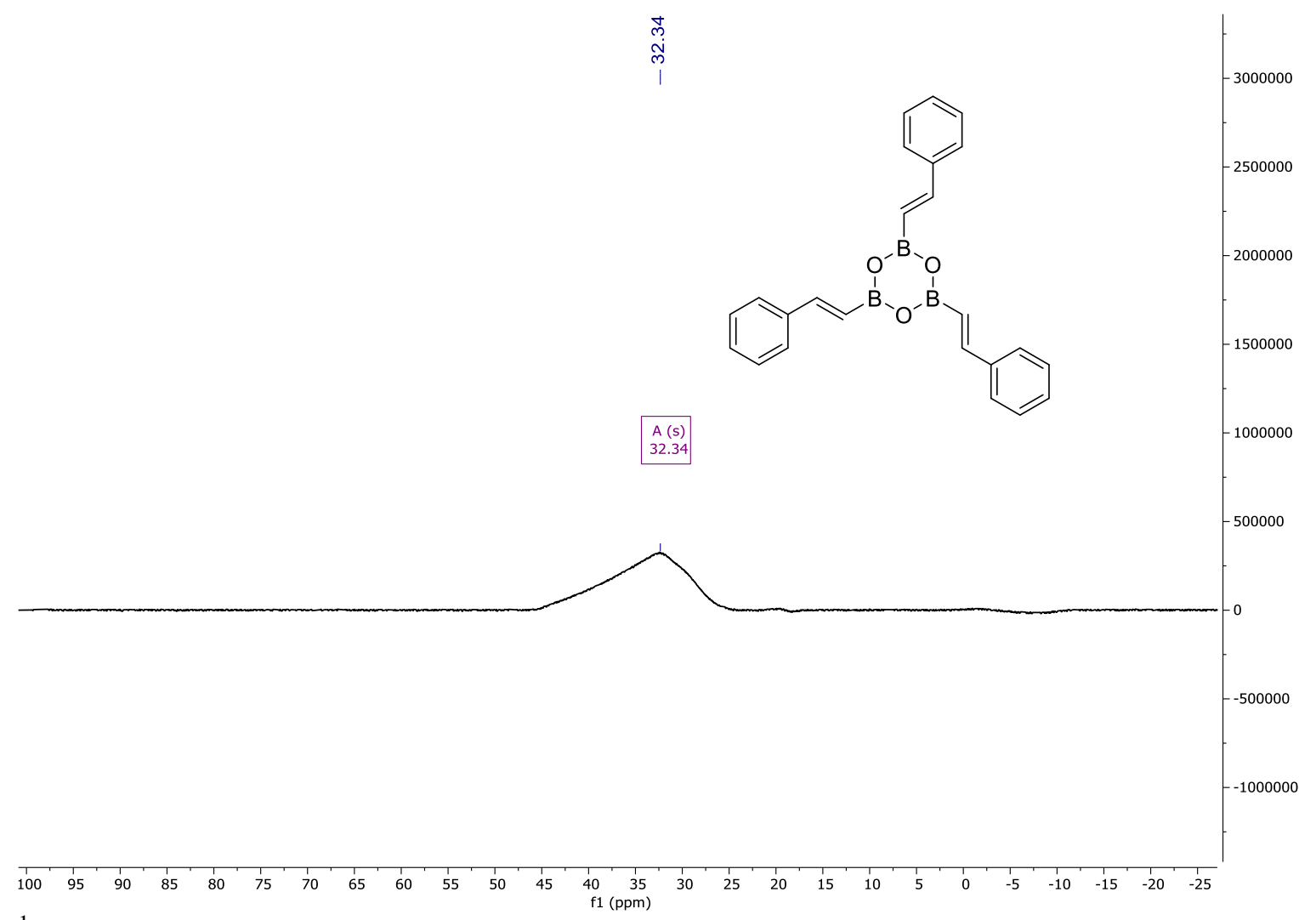

${ }^{1} \mathrm{H}$ NMR (Toluene- $d 8,400 \mathrm{MHz}$ ) of compound $\mathbf{2 e}$

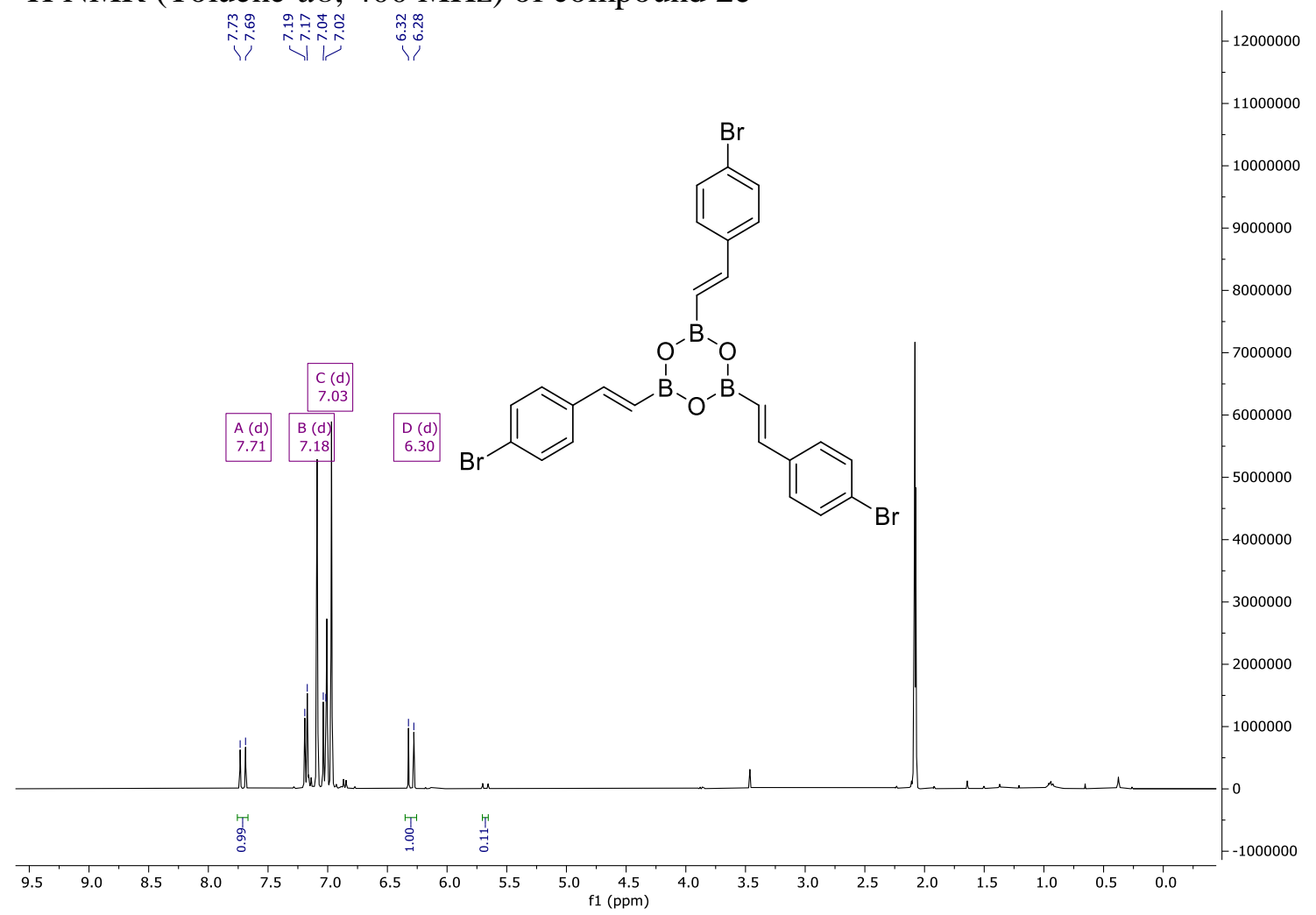

${ }^{11}$ B NMR (Toluene-d8, $128 \mathrm{MHz}$ ) of compound $2 \mathbf{e}$ 


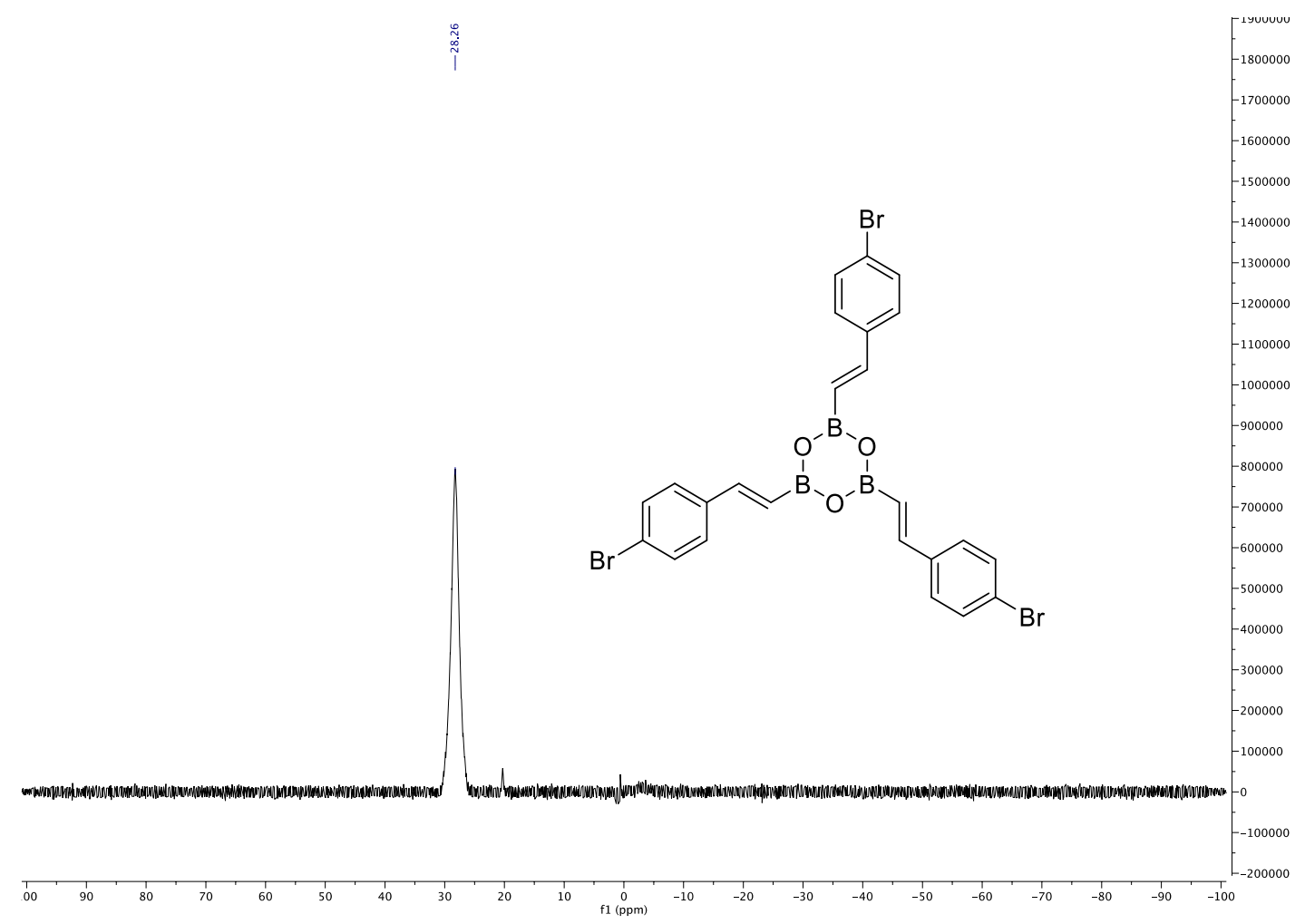

${ }^{1} \mathrm{H}$ NMR (Toluene-d8, $400 \mathrm{MHz}$ ) of compound $\mathbf{2 f}$

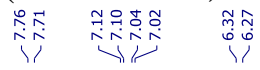
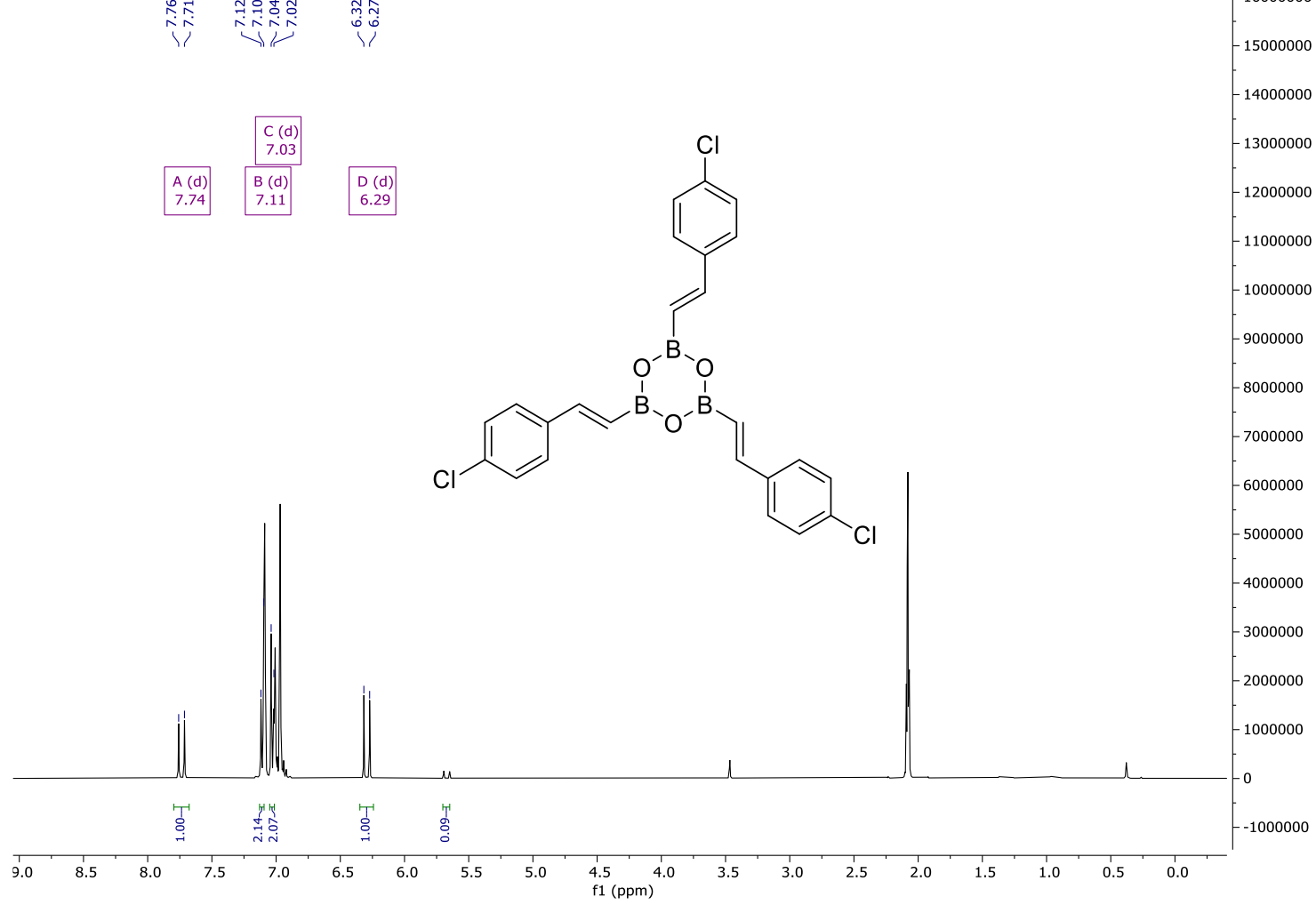

${ }^{11}$ B NMR (Toluene-d8, $128 \mathrm{MHz}$ ) of compound $\mathbf{2 f}$ 


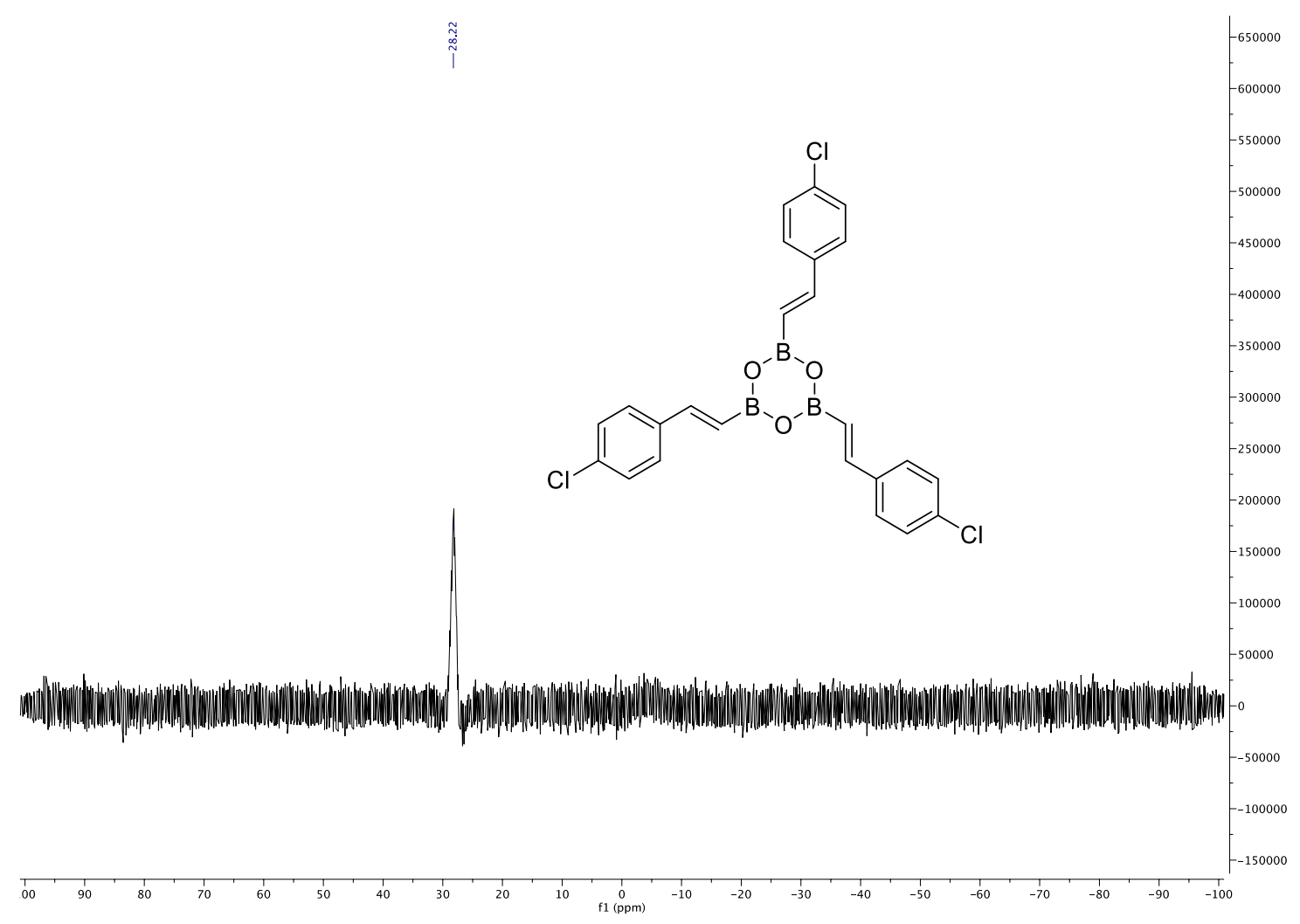

${ }^{1} \mathrm{H}$ NMR (Toluene-d8, $400 \mathrm{MHz}$ ) of compound $\mathbf{2 g}$

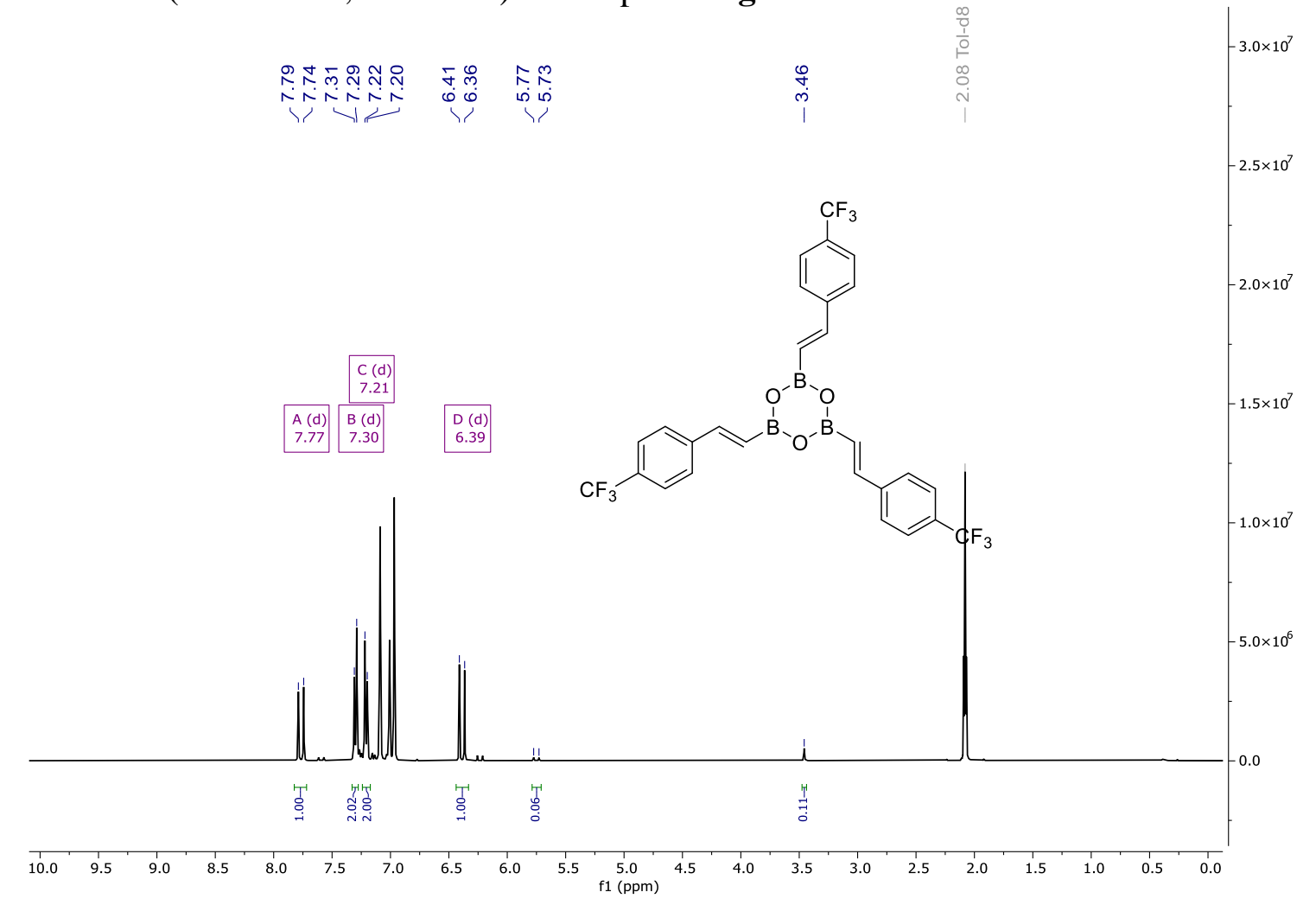


${ }^{11}$ B NMR (Toluene-d8, $128 \mathrm{MHz}$ ) of compound $\mathbf{2 g}$

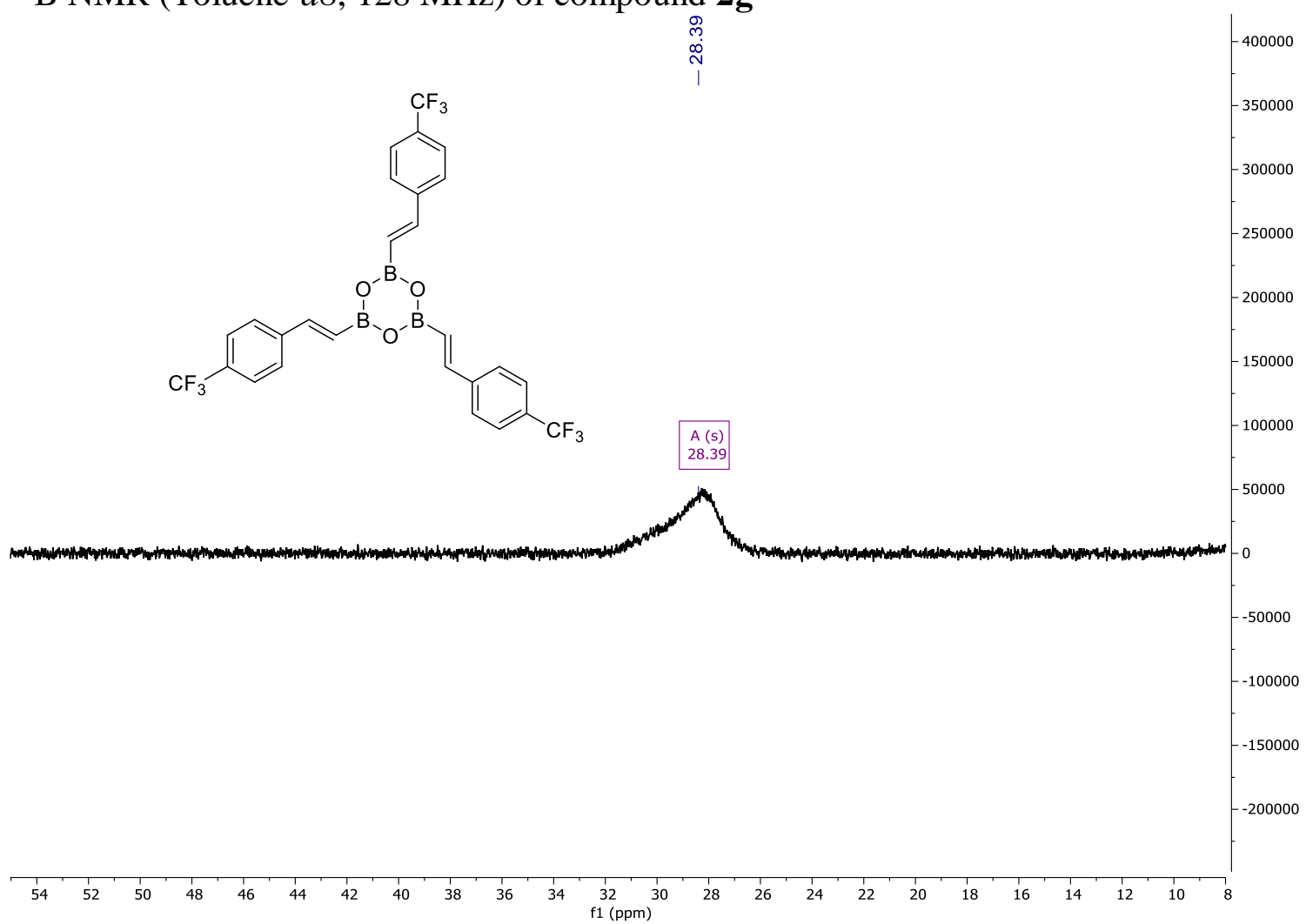

${ }^{1} \mathrm{H}$ NMR (Toluene- $d 8,400 \mathrm{MHz}$ ) of compound $\mathbf{2 h}$

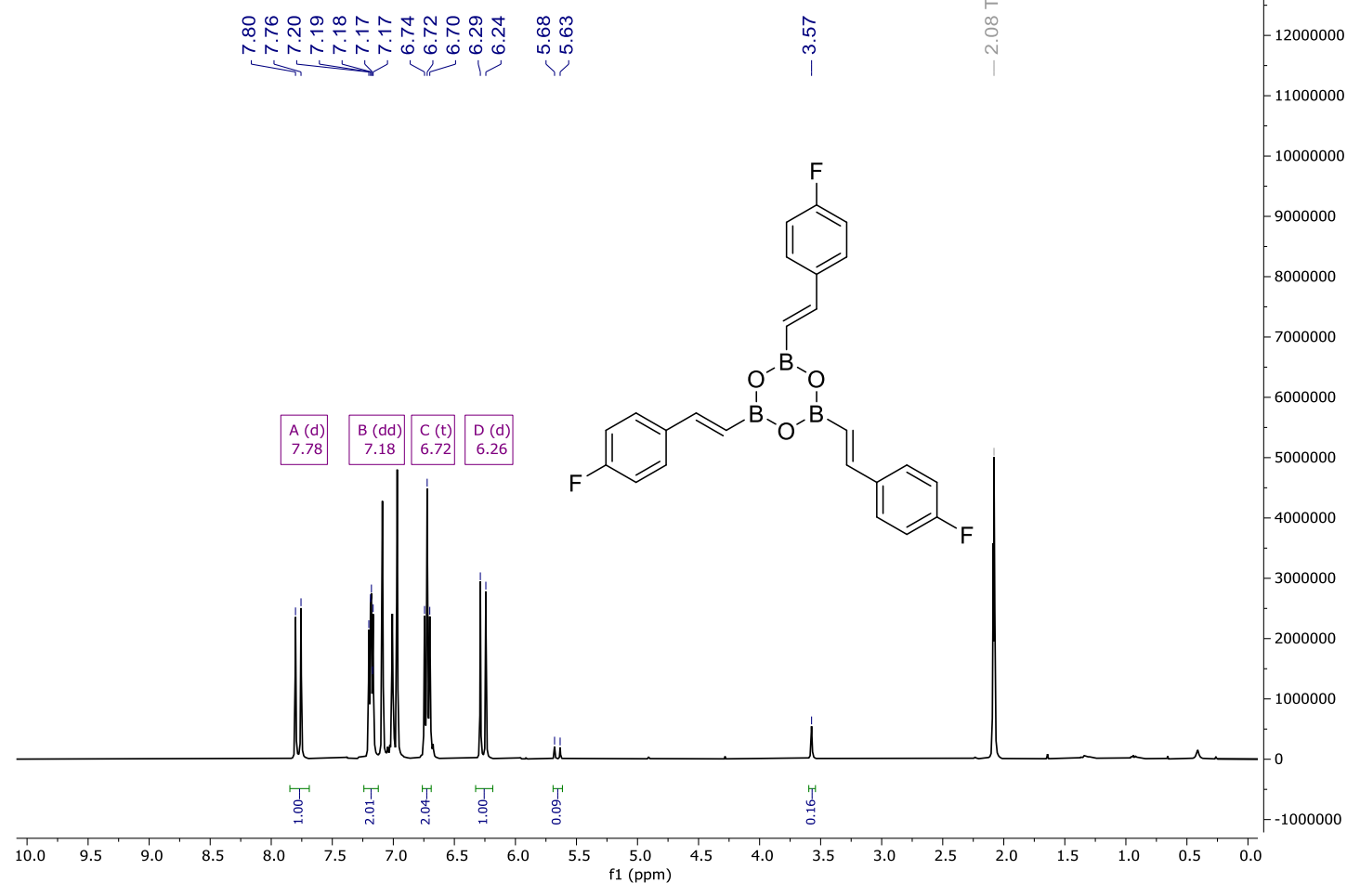

${ }^{11}$ B NMR (Toluene-d8, $128 \mathrm{MHz}$ ) of compound $\mathbf{2 h}$ 


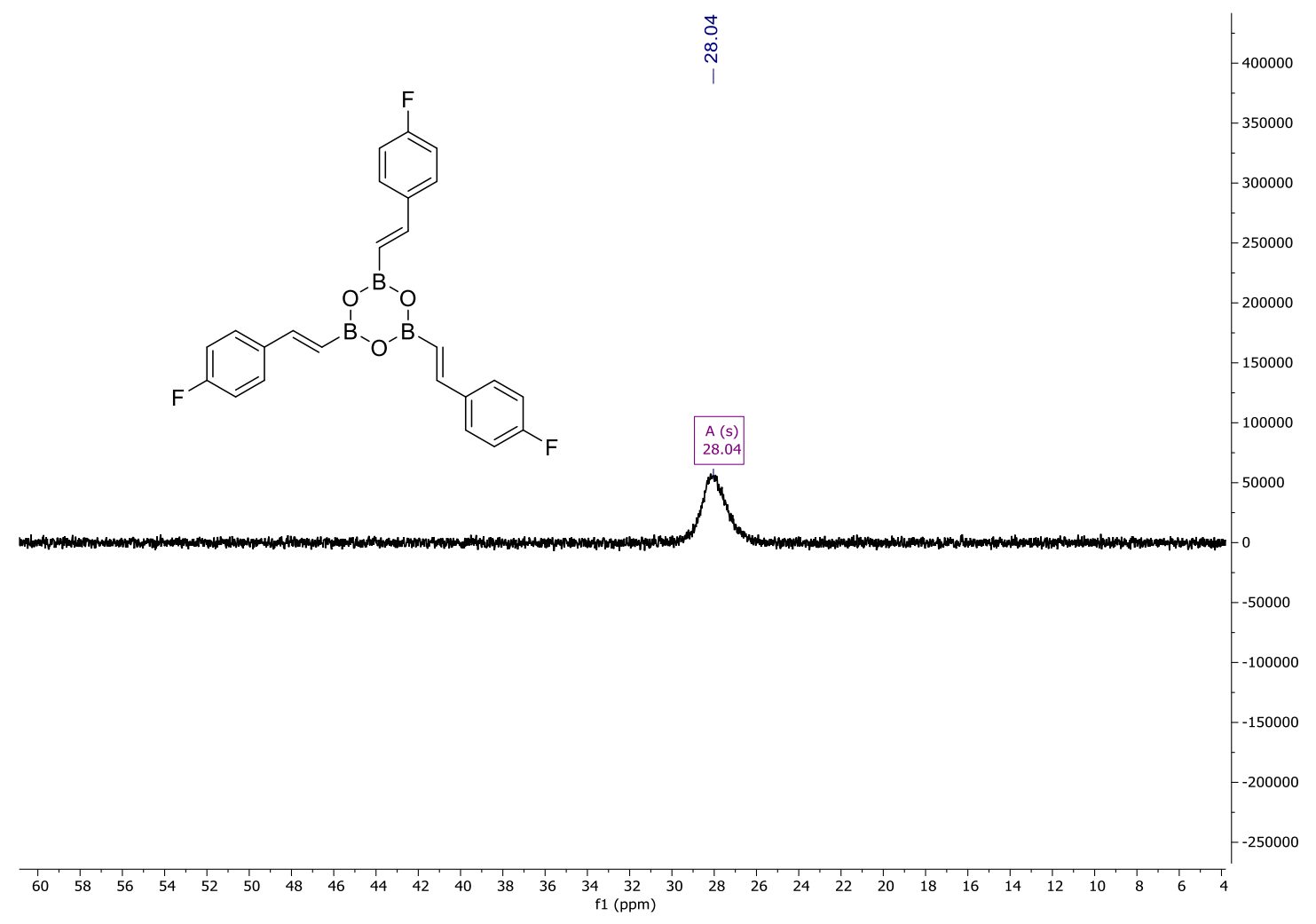

